Prepared in cooperation with Montana Fish, Wildlife, and Parks, U.S. Bureau of Land Management, and the U.S. Environmental Protection Agency

\title{
The Potential Effects of Sodium Bicarbonate, a Major Constituent of Produced Waters from Coalbed Natural Gas Production, on Aquatic Life
}

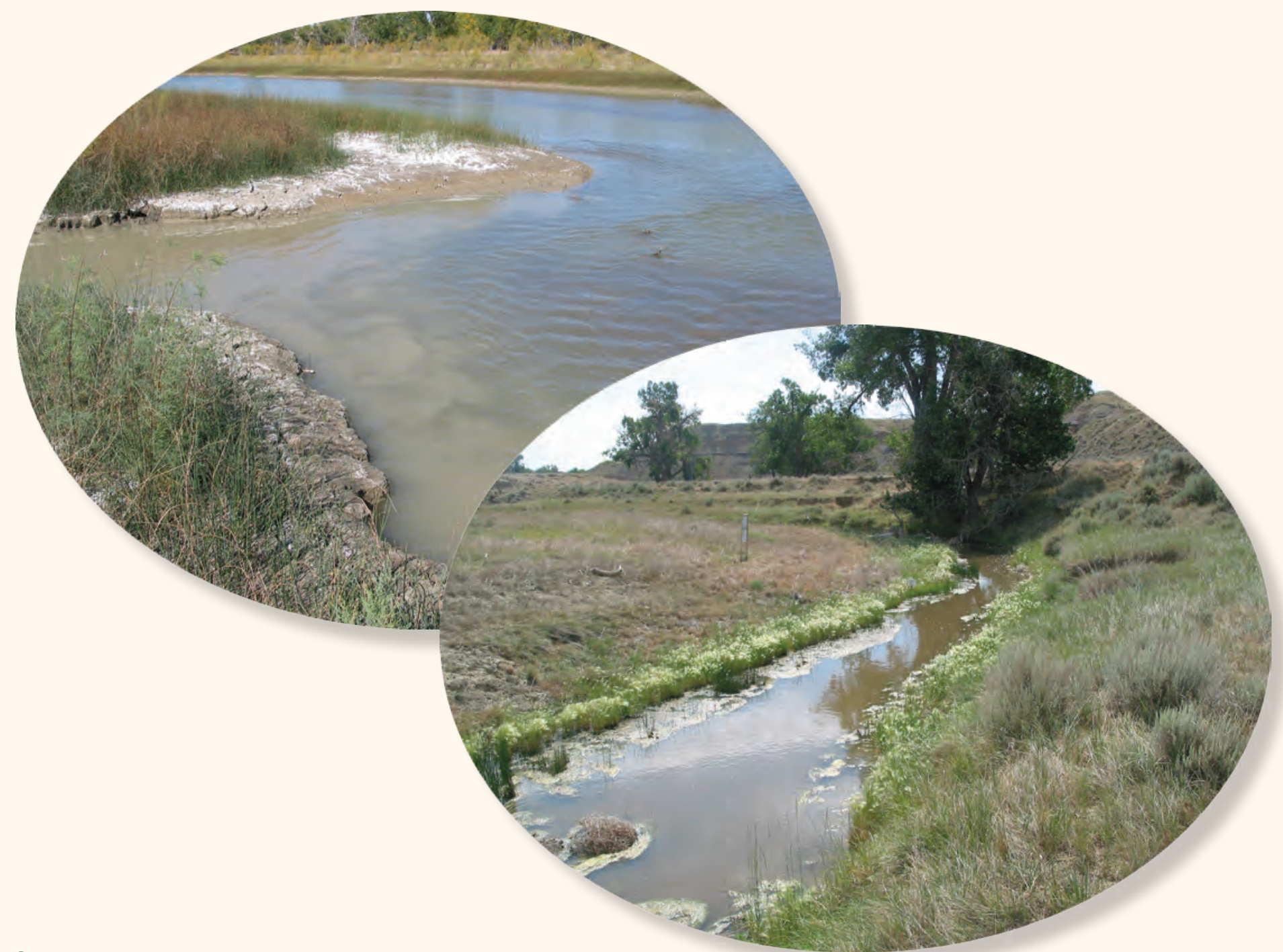

Scientific Investigations Report 2012-5008 


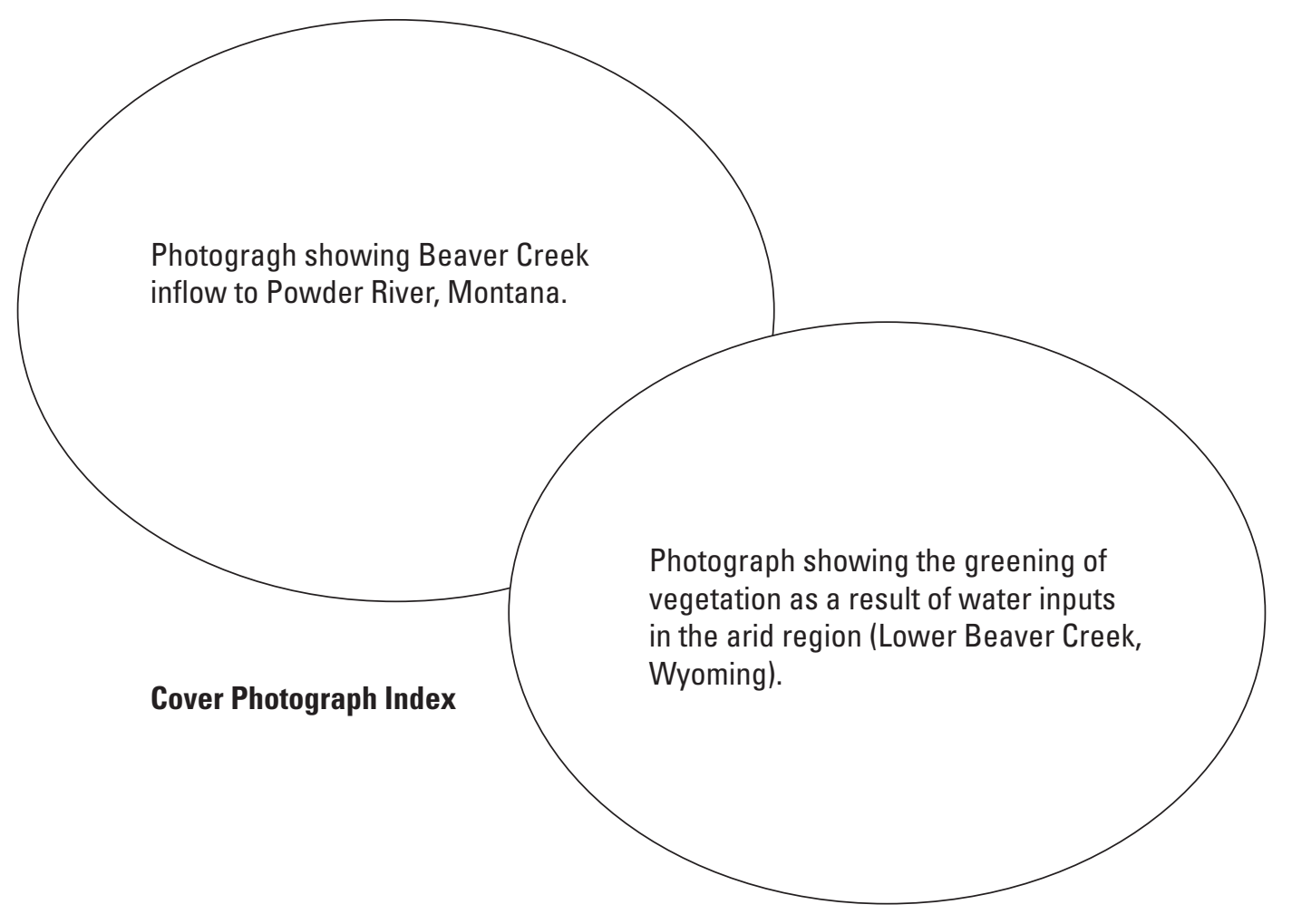




\section{The Potential Effects of Sodium \\ Bicarbonate, a Major Constituent of Produced Waters from Coalbed Natural Gas Production, on Aquatic Life}

Edited by Aïda M. Farag and David D. Harper

Prepared in cooperation with Montana Fish, Wildlife, and Parks, U.S. Bureau of Land Management, and the U.S. Environmental Protection Agency

Scientific Investigations Report 2012-5008 


\section{U.S. Department of the Interior \\ KEN SALAZAR, Secretary \\ U.S. Geological Survey \\ Marcia K. McNutt, Director}

U.S. Geological Survey, Reston, Virginia: 2012

For more information on the USGS - the Federal source for science about the Earth, its natural and living resources, natural hazards, and the environment, visit http://www.usgs.gov or call 1-888-ASK-USGS.

For an overview of USGS information products, including maps, imagery, and publications, visit http://www.usgs.gov/pubprod

Any use of trade, product, or firm names is for descriptive purposes only and does not imply endorsement by the U.S. Government.

Although this report is in the public domain, permission must be secured from the individual copyright owners to reproduce any copyrighted materials contained within this report.

Suggested citation:

Farag, A.M., and Harper, D.D., eds, 2012, The potential effects of sodium bicarbonate, a major constituent from coalbed natural gas production, on aquatic life: U.S. Geological Survey, Scientific Investigations Report 2012-5008, 101 p. 


\section{Acknowledgments}

The research described in this report was a collaborative effort between the U.S. Geological Survey and Montana Fish, Wildlife, and Parks. Thanks to the many others from within and outside of these agencies that participated in the effort.

Connie Boese, University of Wyoming, and staff at the Montana Department of Public Health and Human Services performed chemical measurements. Review comments from Dr. Will Clements, Colorado State University, and J. Russell Hockett, U.S. Environmental Protection Agency, improved the report. Cathy Eakin, Montana Fish, Wildlife, and Parks, provided technical assistance in the field and with laboratory experiments performed in Montana. Elizabeth MacConnell, U.S. Fish and Wildlife Service provided histology analyses. Dr. Joseph Meyer, University of Wyoming, provided input and consultation during initial study designs. Brady Morris, University of Wyoming provided technical assistance with the fathead minnow laboratory experiments and with the presence/absence field work. The crew of the U.S. Fish and Wildlife Service, Jackson National Fish Hatchery, assisted with maintaining cultured organisms in Jackson, Wyoming. Montana, Fish, Wildlife and Parks staff provided sturgeon for experiments. Montana Department of Environmental Quality provided review comments on the draft report. Collaboration with Dave Zaft and additional staff from the Wyoming Game and Fish, Fisheries Research Unit, was instrumental in completion of the presence/absence surveys. The field experiments could not have been conducted without the assistance of the Wyoming Game and Fish Administration and Permitting Office who worked beyond traditional business hours to complete the approval process.

Private landowners were gracious in allowing access to their property for water collection and performing in situ experiments. Thanks especially to John Iberlin and Tom Harriet who allowed access repeatedly over the years. Fidelity Exploration and Production Company allowed access to their property during sample collection from their outflow.

This project would not have been possible without funds provided by the U.S. Bureau of Land Management and the U.S. Environmental Protection Agency. The collaborative process with all of the members of Aquatic Task Group, formed under the Powder River Interagency Working Group, was instrumental as they provided suggestions for study design and focus throughout the project.

Mixing zone studies could not have been completed without Tom Cleasby, Colleague, U.S. Geological Survey who provided assistance in the field and with data interpretations. Brad Mueller, U.S, Geological Survey, provided technical assistance during the fathead minnow experiments. Darren Rhea, U.S. Geological Survey assisted with the presence/absence data collection and compilation. Luke May, U.S. Geological Survey provided assistance with data quality assurance and table construction. The suggestions provided by Kevin Buhl, U.S. Geological Survey were very valuable for the completion of this report. 



\section{Volume Contents}

1. Introduction: The Potential Effects of Sodium Bicarbonate, a Major Constituent of Produced Waters from Coalbed Natural Gas Production, on Aquatic Life .......................1

2. The Acute Toxicity of Sodium Bicarbonate Defined Under Laboratory Conditions ..........9

3. The Chronic Toxicity of Sodium Bicarbonate Defined Under Laboratory Conditions .....39

4. The Potential Toxicity Defined In Situ and Streamside in the Powder River and Tongue River Basins.

5. Presence/Absence of Native and Non-native Species in the Powder and Tongue River Watersheds . .79

6. Summary and Sample Calculations for Water-Quality Criteria.......................................93 


\section{Conversion Factors, Abbreviations, and Datums}

\begin{tabular}{lll}
\multicolumn{1}{c}{ SI to Inch/Pound } & \multicolumn{1}{c}{ To obtain } \\
\hline \multicolumn{1}{c}{ Multiply } & \multicolumn{1}{c}{ By } & \\
\hline centimeter $(\mathrm{cm})$ & 0.3937 & inch (in.) \\
millimeter $(\mathrm{mm})$ & 0.03937 & inch (in.) \\
meter $(\mathrm{m})$ & 3.281 & foot (ft) \\
kilometer $(\mathrm{km})$ & 0.6214 & mile (mi) \\
kilometer $(\mathrm{km})$ & 0.5400 & mile, nautical (nmi) \\
meter $(\mathrm{m})$ & 1.094 & yard (yd) \\
\hline & \multicolumn{1}{c}{ Volume } & \\
\hline liter $(\mathrm{L})$ & 33.82 & ounce, fluid (fl. oz) \\
liter $(\mathrm{L})$ & 2.113 & pint (pt) \\
liter $(\mathrm{L})$ & 1.057 & quart (qt) \\
liter $(\mathrm{L})$ & 0.2642 & gallon (gal) \\
microliter $(\mu \mathrm{L})$ & $3.382 \times 10^{-5}$ & ounce, fluid (fl. oz) \\
& $2.113 \times 10^{-5}$ & pint (pt) \\
& $1.057 \times 10^{-5}$ & quart (qt) \\
liter $(\mathrm{L})$ & $0.2642 \times 10^{-5}$ & gallon (gal) \\
\hline
\end{tabular}

Temperature in degrees Celsius $\left({ }^{\circ} \mathrm{C}\right)$ may be converted to degrees Fahrenheit $\left({ }^{\circ} \mathrm{F}\right)$ as follows:

$$
{ }^{\circ} \mathrm{F}=\left(1.8 \times{ }^{\circ} \mathrm{C}\right)+32
$$

Temperature in degrees Fahrenheit $\left({ }^{\circ} \mathrm{F}\right)$ may be converted to degrees Celsius $\left({ }^{\circ} \mathrm{C}\right)$ as follows:

$$
{ }^{\circ} \mathrm{C}=\left({ }^{\circ} \mathrm{F}-32\right) / 1.8
$$

Vertical coordinate information is referenced to the North American Vertical Datum of 1988 (NAVD 88).

Horizontal coordinate information is referenced to the North American Datum of 1983 (NAD 83).

Altitude, as used in this report, refers to distance above the vertical datum.

Specific conductance is given in microsiemens per centimeter at 25 degrees Celsius $\left(\mu \mathrm{S} / \mathrm{cm}\right.$ at $\left.25^{\circ} \mathrm{C}\right)$.

Concentrations of chemical constituents in water are given either in milligrams per liter ( $\mathrm{mg} / \mathrm{L}$ ) or micrograms per liter $(\mu \mathrm{g} / \mathrm{L})$.

NOTE TO USGS USERS: Use of hectare (ha) as an alternative name for square hectometer $\left(\mathrm{hm}^{2}\right)$ is restricted to the measurement of small land or water areas. Use of liter (L) as a special name for cubic decimeter $\left(\mathrm{dm}^{3}\right)$ is restricted to the measurement of liquids and gases. No prefix other than milli should be used with liter. Metric ton ( $t$ ) as a name for megagram $(\mathrm{Mg})$ should be restricted to commercial usage, and no prefixes should be used with it. 


\section{Introduction: The Potential Effects of Sodium Bicarbonate, a Major Constituent of Produced Waters from Coalbed Natural Gas Production, on Aquatic Life}

By Aïda M. Farag, Don Skaar, and David D. Harper

Chapter 1 of

The Potential Effects of Sodium Bicarbonate, a Major Constituent of Produced Waters from Coalbed Natural Gas Production, on Aquatic Life

Edited by Aïda M. Farag and David D. Harper

Prepared in cooperation with Montana Fish, Wildlife, and Parks, U.S. Bureau of Land Management, and the U.S. Environmental Protection Agency

Scientific Investigations Report 2012-5008 


\section{Contents}

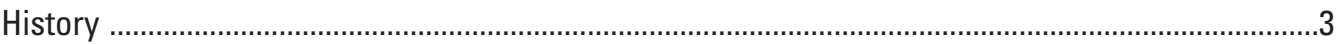

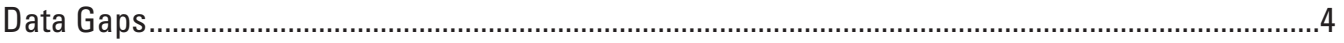

General Study Design

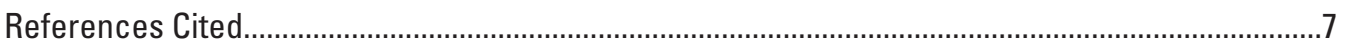

\section{Figures}

1-1. Diagram showing approaches needed to define the potential effects of $\mathrm{NaHCO}_{3}$ (sodium bicarbonate) at both the individual and population levels, and to move the understanding from the laboratory to the field.

1-2. Map showing the locations of the Tongue and Powder River Basins in the Tongue River/Powder River watershed of eastern Wyoming and Montana.....

\section{Table}

1-1. Experiments completed to achieve minimum requirements for the establishment of a water-quality criterion for sodium bicarbonate. 


\title{
Introduction: The Potential Effects of Sodium Bicarbonate, a Major Constituent of Produced Waters from Coalbed Natural Gas Production, on Aquatic Life
}

\author{
By Aïda M. Farag', Don Skaar², and David D. Harper ${ }^{1}$
}

\section{History}

A substantial expansion in coalbed natural gas production has taken place in the western United States. The production water from coalbed natural gas extraction contains many constituents. For some of these constituents, such as aluminum, ammonia, copper, lead, cadmium and zinc, the U.S. Environmental Protection Agency has established aquatic life criteria, and it is therefore possible to evaluate their risk to aquatic life. However, of the major ions associated with produced waters (sodium $\left[\mathrm{Na}^{+}\right]$, calcium $\left[\mathrm{Ca}^{2+}\right]$, magnesium $\left[\mathrm{Mg}^{2+}\right]$, potassium $\left[\mathrm{K}^{+}\right]$, chloride $\left[\mathrm{Cl}^{-}\right]$, bicarbonate $\left[\mathrm{HCO}_{3}^{-}\right]$, sulfate $\left[\mathrm{SO}_{4}^{2-}\right]$ ), chloride is the only one with an established aquatic life criterion (U.S. Environmental Protection Agency, 1988). Previous research determined that different ions (and salts they form) induce varying degrees of toxicity to aquatic life (for example, Young, 1923; Mossier, 1971; Nelson, 1968; Held and Peterka, 1974; Rawson and Moore, 1944). Mount and others (1997) completed more than a thousand acute experiments and developed a multiple regression model that described the toxicity of common ions in various combinations to zooplankton and fathead minnows (Pimephales promelas, FHM). One of the most pivotal findings of Mount and others (1997) was that all major ions have a lethal concentration, and the toxicity of a mixture of salts is generally equivalent to the additive toxicity of the individual salts.

During a toxicity identification evaluation, Boelter and others (1992) defined salinity as the cause of toxicity in an oil field discharge collected in northeastern Wyoming. The Mount and others (1997) study defined the salt most likely to be toxic to aquatic life that comes from coalbed natural gas production water as sodium bicarbonate $\left(\mathrm{NaHCO}_{3}\right)$. A lethal concentration of $\mathrm{NaHCO}_{3}$ in three 96-hour experiments caused a 50-percent mortality (LC50) of less than 850 milligrams per liter $(\mathrm{mg} / \mathrm{L})$ for this salt, which is below the mean concentration of $\mathrm{NaHCO}_{3}$ discharged from some production sites, for example $1,335 \mathrm{mg} \mathrm{NaHCO} / \mathrm{L}$ at the $\mathrm{CX}$ Ranch production field near

${ }^{1}$ USGS, Columbia Environmental Research Center, Jackson Field Research Station, Jackson, Wyoming.

${ }^{2}$ Montana Fish, Wildlife, and Parks, Helena, Montana.
Decker, Montana, not shown (Bauder, 2002). Few other published studies have investigated the acute toxicity of this salt. Beatty (1959) experimented with this salt on rainbow trout and determined that 65 percent of the fish were killed within a week at a $\mathrm{NaHCO}_{3}$ concentration of $1,000 \mathrm{mg} / \mathrm{L}$. However, Beatty (1959) used reconstituted water (distilled water plus $\mathrm{NaHCO}_{3}$ ) in the experiments, without the addition of other salts that are necessary for normal physiological functioning; the results, therefore, are not a reliable predictor of effects of this salt in natural waters.

The problem of interpreting the scant $\mathrm{NaHCO}_{3}$ acute toxicity data available is exaggerated by the absence of adequate laboratory studies on the chronic effects of $\mathrm{NaHCO}_{3}$. A few field studies exist that fall short of clearly demonstrating the effects of $\mathrm{NaHCO}_{3}$. For example, McCarraher and Thomas (1968) studied the tolerance of FHM in saline lakes in the sand hills of Nebraska where sodium bicarbonate was the dominant salt. Using in situ bioassays, they determined that lakes in which the minnows were able to survive for 6 months or more had bicarbonate + carbonate concentrations that averaged $1,061 \mathrm{mg} / \mathrm{L}$ and ranged from 336 to $1,979 \mathrm{mg} / \mathrm{L}$. Lakes in which the minnows did not survive 6 months contained bicarbonate and carbonate concentrations that averaged $4,282 \mathrm{mg} / \mathrm{L}$ and ranged from 1,410 to $20,760 \mathrm{mg} / \mathrm{L}$. Minnows in the lake with $1,410 \mathrm{mg} / \mathrm{L}$ bicarbonate and carbonate began to die within 60 days and incurred total mortality within 80 days. Total alkalinities of 1,800 and 2,000 mg/L were defined as thresholds for successful reproduction in two lakes in which more in depth studies were completed by McCarraher and Thomas (1968). Galat and others (1985) studied the Lahonton cutthroat trout (Oncorhynchus clarkii henshawi) in saline lakes in Nevada and Oregon, not shown. This species is well known for its ability to tolerate saline conditions, an ability perhaps developed throughout the millennia from living in Pleistocene Lake Lahonton, not shown. Histological changes in the tissues of these fish were studied, and in lakes with a range of alkalinity from 60 to $3,500 \mathrm{mg}$ calcium carbonate $\left(\mathrm{CaCO}_{3}\right) / \mathrm{L}$, there was a correlation between $\mathrm{HCO}_{3}$, carbon dioxide $\left(\mathrm{CO}^{2-}\right), \mathrm{Cl}^{-}$concentrations and gill cell hyperplasia.

Neither the McCarraher and Thomas (1968) nor the Galat and others (1985) field studies reported in the literature are 
suitable to develop chronic threshold concentrations for the effects of $\mathrm{NaHCO}_{3}$. The development of chronic threshold concentrations for $\mathrm{NaHCO}_{3}$ effects requires experimenting with a salt intolerant species and the measurement of sublethal effects on the most sensitive lifestage. Moreover, no other regulatory tools currently are available to assure long-term protection of aquatic life exposed to $\mathrm{NaHCO}_{3}$. Even whole effluent testing (WET) of coalbed natural gas well discharge is focused only on the measurement of and protection from acute toxicity, and WET testing results will certainly not be adequate for establishing Total Maximum Daily Loads (TMDLs) for drainages with coalbed natural gas production.

\section{Data Gaps}

Multiple approaches are needed to more completely define the potential toxicity of sodium bicarbonate on aquatic life. Approaches that define the potential effects at levels that range from the individual to the population and that work to explain implications as research moves from the laboratory to the field, can assist in better understanding the overall potential for $\mathrm{NaHCO}_{3}$ to adversely affect aquatic resources (fig. 1-1).

It is the intersection of information gathered as we move from the individual organism to effects at the population level, and as we move from the laboratory to the field, that guide an understanding and ability to predict the overall effects of $\mathrm{NaHCO}_{3}$ in real world conditions. This wide range of understanding also would give resource managers a science-based

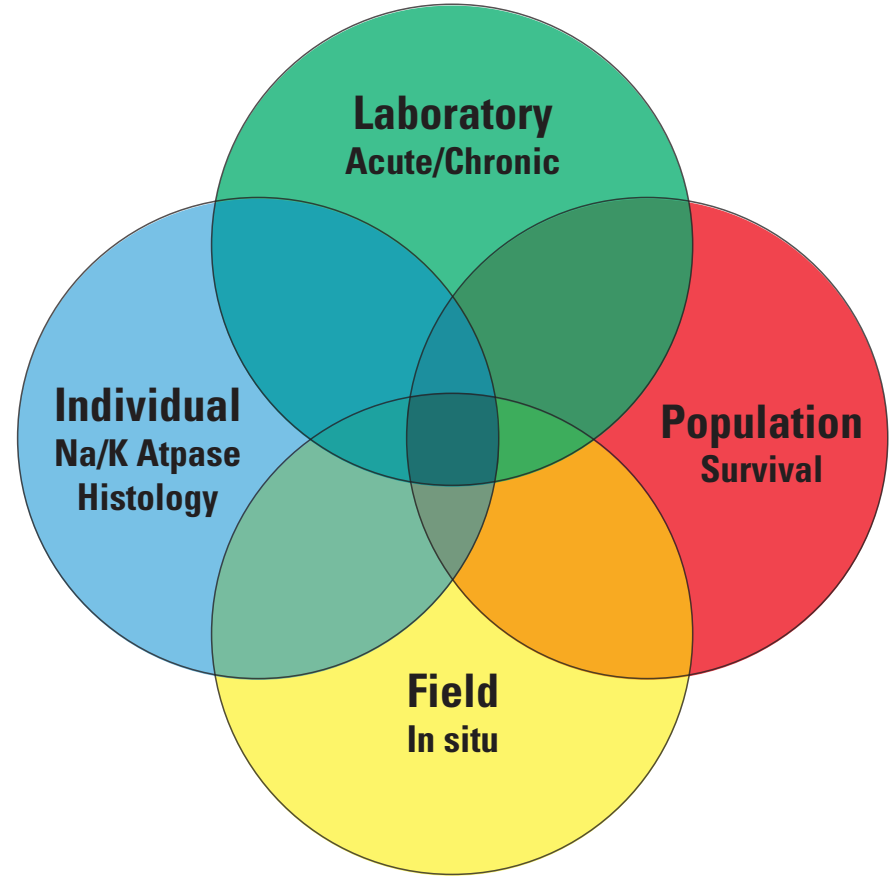

Figure 1-1. Approaches needed to define the potential effects of $\mathrm{NaHCO}_{3}$ (sodium bicarbonate) at both the individual and population levels, and to move the understanding from the laboratory to the field. confidence upon which to employ adaptive management decisions.

To fine tune the general concept provided above, several types of experiments could be employed. First, laboratory experiments using water to mimic field conditions will define the toxicity attributable to $\mathrm{NaHCO}_{3}$. A series of acute and chronic laboratory experiments with multiple aquatic species, especially those present in areas of concern, would provide needed input for no-effect level (NOEL) determinations for $\mathrm{NaHCO}_{3}$. Second and concurrently, a field survey to define the presence/absence of aquatic species would be necessary and could define the transferability of laboratory data to the field. Third, in situ bioassays could further ground-truth laboratory data and refine information about the lifestage sensitivity in field conditions. Fourth, experiments with mixing zone waters could define the extent of potential toxicity in the areas of concern. These data could assist managers as they decide on the extent of the potential for effect concentrations to be reached in a watershed.

Additionally, 60-day studies that begin with fertilization will span the egg, hatch, sac-fry and swim-up stages of selected fish species. Therefore, experiments of this length will provide a chronic exposure of fish during their sensitive early life stages. In addition to survival, sublethal effects that can be measured to define chronic thresholds may include effects on growth, histology, whole-body or plasma ion concentrations and sodium-potassium adenosine triphosphatase $(\mathrm{Na} / \mathrm{K}$ ATPase) in early lifestage fish that have been exposed to $\mathrm{NaHCO}_{3}$ under chronic conditions. All of these parameters studied together may provide a pattern of events to explain changes in growth. For example, upsets in enzyme levels may result in ionic imbalance with histological changes at the gill to follow. These physiological changes, ultimately, may be manifested as growth reductions in the laboratory. As a result, not only will chronic no-effect levels be defined, attempts will be made to explain the mechanisms of toxicity that are involved.

A TMDL dataset that includes $\mathrm{NaHCO}_{3}$ would not be complete without field validation of the laboratory assays. The presence/absence of native and game species of fish could ground truth the predicted no-effect levels established with laboratory studies. Furthermore, Clearwater and others (2002) suggest that an increase in salinity from product waters of coalbed methane production might increase salinity of the upper reaches of the Tongue River. The authors suggest increased salinity might affect the habitat quality of these reaches for fishes. Therefore, adequate baseline data of species distribution are needed in this watershed. The baseline data also would document the presence/absence of the species chosen for use during laboratory experiments.

Field toxicity experiments are important for multiple reasons. First, when supplemented with the proper water-quality information, in situ bioassays can further ground truth the laboratory investigations. Assessments can be made to define whether or not the no-effect levels of $\mathrm{NaHCO}_{3}$ determined in the laboratory are similar to those found at sites of critical importance. The water quality at various field sites may 
have additional constituents, so it is important that multiple constituents in the water be monitored during any in situ study. Second, in situ bioassays can be used to monitor changes in future survivability at sites in question. Therefore, in situ bioassays could alert managers to issues of degraded water quality or highlight areas where sites have been remediated and are more hospitable to fish survival.

\section{General Study Design}

The approaches described in Data Gaps above were used to determine the potential effects of $\mathrm{NaHCO}_{3}$, a major constituent of coalbed natural gas production water on aquatic life. Water qualities in the Tongue and Powder Rivers in Montana and Wyoming (fig. 1-2) were simulated and used in laboratory studies, and field studies were completed at sites along tributaries and the main stem of these rivers. The series of acute and chronic studies completed in the laboratory (table 1-1) span multiple species and phyla, and addresses requirements defined by the U.S. Environmental Protection Agency (Stephan and others, 1995) for establishment of waterquality criteria.

This effort was completed to expand upon the limited 2002 knowledge base related to the potential effects of $\mathrm{NaHCO}_{3}$. To achieve balance between beneficial use of scarce water resources and the protection of aquatic life, managers need a broad range of fundamental science on which to base their far reaching decisions. The information gathered and the interpretations provided in the following chapters should provide managers with some needed information as they implement adaptive management practices.

The focus of this research was $\mathrm{NaHCO}_{3}$, a compound that is a major constituent of coalbed natural gas produced waters in the Tongue and Powder River Basins. This compound was added during controlled experiments, and for this reason, we chose to formulate sample criteria and discussions of potential toxicity with concentrations of the compound $\mathrm{NaHCO}_{3}$. However, criteria often are established for single elements or ions, in this case most likely $\mathrm{HCO}_{3}^{-}$as the toxic fraction of the compound $\mathrm{NaHCO}_{3}$ (Mount and others, 1997). Therefore, $\mathrm{HCO}_{3}{ }_{3}^{-}$information has been provided for use if derivations with this single element are preferred. The sample criteria could also be calculated as alkalinity because it is an easily measured water chemistry property that is expressed as $\mathrm{mg}$ $\mathrm{CaCO}_{3} / \mathrm{L}$, but defines the amount of $\mathrm{HCO}_{3}{ }^{-}$in a sample with a pH less than 8.3 (American Public Health Association, 1975). In the Standard Method (American Public Health Association, 1975) for alkalinity measurement, a sample with $\mathrm{pH}$ greater than 8.3 is titrated to $\mathrm{pH}=8.3$ with sulfuric acid. The amount of titrant used translates into $\mathrm{mg} \mathrm{CaCO}_{3} / \mathrm{L}$ in the sample. The titration is continued until $\mathrm{pH}=4.5$ and the additional milliliters $(\mathrm{mL})$ of titrant used translates into the amount of $\mathrm{HCO}_{3}^{-}$in the sample. The total amount of titrant used expresses the total alkalinity. Data has been provided for total alkalinity, $\mathrm{HCO}_{3}^{-}$ and $\mathrm{CO}_{3}$ measurements. With this information, managers can use this dataset to interpret the potential toxicity expressed in

Table 1-1. Experiments completed to achieve minimum requirements for the establishment of a water-quality criterion for sodium bicarbonate. U.S. Environmental Protection Agency requirement/guidance taken from Stephen and others (1995).

U.S. Environmental Protection Agency requirement/guidance acute criteria

One plant species
Eight animal species
-Three families of fish from Osteichthyes, including one
from Salmonidae, and one from a warmwater species

Planctonic crustacean

Benthic crustacean

Insect

Non-Insect phylum

Insect or any other phylum not already represented

One fish species

One invertebrate species

One acutely sensitive freshwater species

\section{Experiments completed during this project}

${ }^{1}$ Selenastrum capricornutum.

Salmonidae-cutthroat trout.

Percidae-walleye.

Catastomidae-white sucker.

Escoidae-northern pike.

Cyprinidae-fathead minnow (warmwater).

Centrarchidae-largemouth bass (warmwater).

Acipenseridae-pallid and shovelnose sturgeon (warmwater).

Ceriodaphnia (literature exists for Ceriodaphnia and Daphnia).

Gammarus or Hyalella.

Chironomus.

Annelid-Tubifex.

African clawed frog-Xenopus laevis.

White sucker.

Fathead minnows.

Ceriodaphnia

Freshwater mussel.

${ }^{1}$ Results not available at time of publication. Also completed: (1) 96-hour in situ experiments during two different years at six different sites with pallid sturgeon and fathead minnow; (2) 96-hour laboratory experiments with pallid sturgeon exposed to water collected from several of the field sites; (3) 96-hour laboratory experiments with fathead minnow exposed to site water from coal bed natural gas mixing zones. 


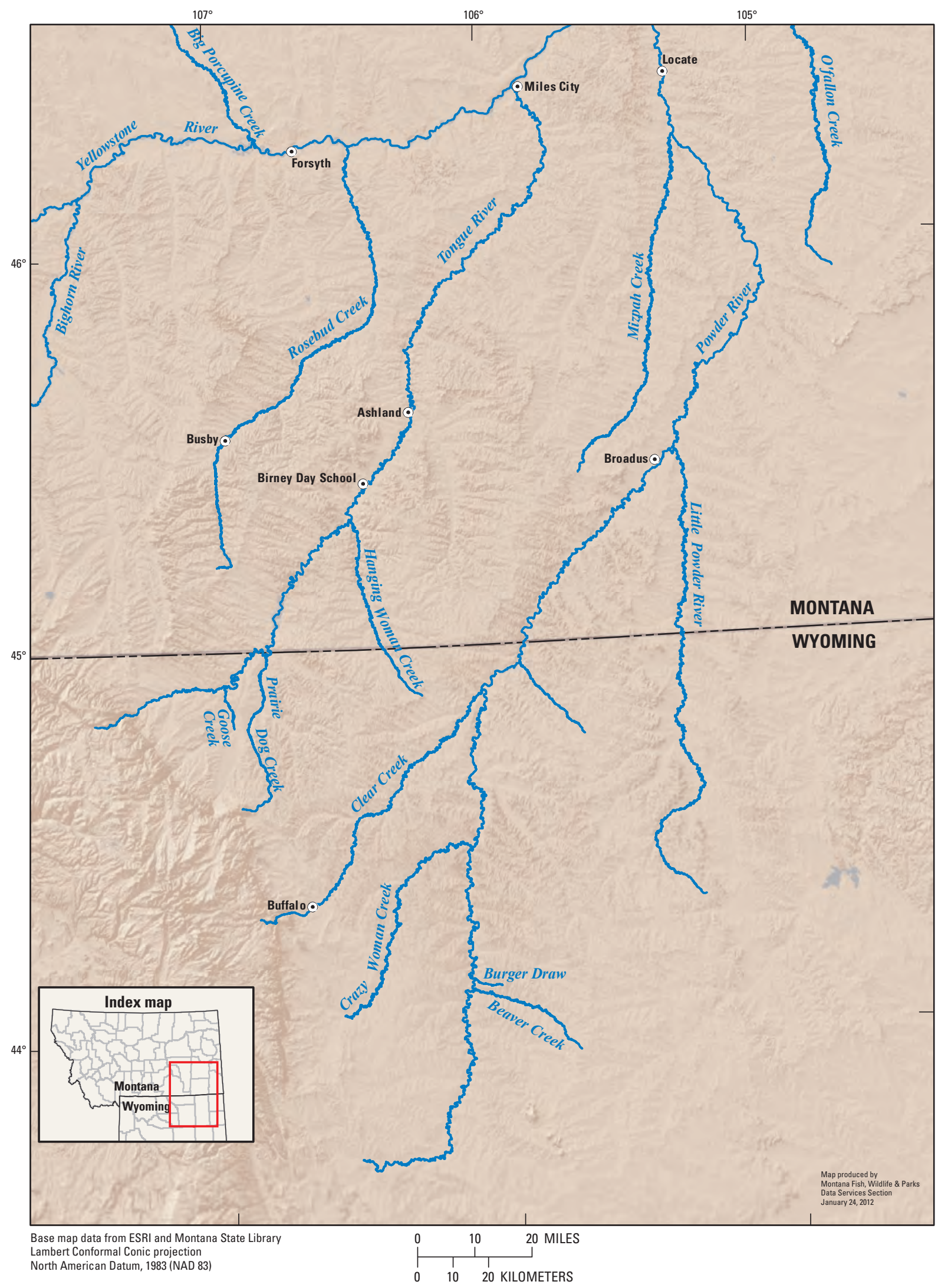

Figure 1-2. The locations of the Tongue and Powder River Basins in the Tongue River/Powder River watershed of eastern Wyoming and Montana. 
the manner that best suits their needs. Conductivity is another measurement that increases as concentrations of $\mathrm{NaHCO}_{3}$ increase in the water column. Measurements of conductivity also have been provided, especially for field experiments, should managers want to express toxicity in the context of conductivity measured as microsiemens per centimeter $(\mu \mathrm{S} /$ $\mathrm{cm})$.

\section{References Cited}

American Public Health Association Standard Methods, 1975, Alkalinity titration method for water and wastewater (14th ed.): Washington, D.C., American Public Health Association, 14th edition, p. 278.

Bauder, J.W., 2002, Quality and characteristics of saline and sodic water affect irrigation suitability: Montana State Univeristy, accessed April 10, 2010, at http://waterquality. montana.edu/, $7 \mathrm{p}$.

Beatty, D.D., 1959, An experimental study of the toxicity of sodium bicarbonate, sodium chloride, and sodium sulfate to rainbow trout: Laramie, University of Wyoming, Master's Thesis, $69 \mathrm{p}$.

Boelter, A.M., Lamming, F.N., Farag, A.M., and Bergman, H.L., 1992, Environmental effects of saline oil-field discharges on surface waters: Environmental Toxicology and Chemistry, v. 11, p. 1,187-1,195.

Clearwater, S.J., Morris, B.A., and Meyer, J.S., 2002, A comparison of coalbed methane product water quality versus surface water quality in the Powder River Basin of Wyoming, and an assessment of the use of standard aquatic testing organisms for evaluating the potential effects of coalbed methane product waters: Laramie, University of Wyoming, Department of Zoology and Physiology, Report to the Wyoming Department of Environmental Quality, 131 p.

Galat, D.L., Post, G., Keefe, T.J., and Bouck, G.R., 1985, Histological changes in the gill, kidney and liver of Lahonton cutthroat trout, Salmo clarki henshawi, living in lakes of different salinity-alkalinity: Journal of Fish Biology, v. 27, p. 533-552.
Held, J.W., and Peterka, J.J., 1974, Age, growth, and food habits of the fathead minnow, Pimephales promelas, in North Dakota saline lakes: Transactions of the American Fisheries Society., v. 103, p. 743-756.

McCarraher, D.B., and Thomas, R., 1968, Some ecological observations on the fathead minnow, Pimephales promelas, in the alkaline waters of Nebraska: Transactions of the American Fisheries Society, v. 97, p. 52-55.

Mount, D.R., Gulley, D.D., Hockett, J.R., Garrison, T.D., and Evans, J.M., 1997, Statistical models to predict the toxicity of major ions to Ceriodaphnia dubia, Daphnia magna, and Pimephales promelas (fathead minnows): Environmental Toxicology and Chemistry, v. 16, p. 2,009-2,019.

Mossier, J.N., 1971, The effect of salinity on the eggs and sac fry of the fathead minnow (Pimephales promelas promelas), northern pike (Esox lucius) and walleye (Stizostedion vitreum vitreum): Fargo, North Dakota State University, Ph.D. dissertation, $47 \mathrm{p}$.

Nelson, J.S., 1968, Salinity tolerance of brook sticklebacks, Culaea inconstans, freshwater ninespine sticklebacks, Pungitius pungitius, and freshwater fourspine sticklebacks, Apeltes quadracus: Canadian Journal of Zoology, v. 46, p. 663-667.

Rawson, D.S., and Moore, J.E., 1944, The saline lakes of Saskatchewan: Canadian Journal of Research, v. 22, p. 141-201.

U.S. Environmental Protection Agency, 1988, Ambient water quality criteria for chloride: Cincinnati, Environmental Monitoring Systems Laboratory Office of Research and Development, U.S. Environmental Protection Agency EPA 440/5-88-001, 39 p.

Young, R.T, 1923, Resistance of fish to salts and alkalinity: American Journal of Physiology, v. 63, p. 373-388. 



\section{The Acute Toxicity of Sodium Bicarbonate Defined Under Laboratory Conditions}

By David D. Harper, Aïda M. Farag, Don Skaar, and Trevor Selch

\section{Chapter 2 of}

The Potential Effects of Sodium Bicarbonate, a Major Constituent of Produced Waters from Coalbed Natural Gas Production, on Aquatic Life

Edited by Aïda M. Farag, and David D. Harper

Prepared in cooperation with Montana Fish, Wildlife, and Parks, U.S. Bureau of Land Management, and the U.S. Environmental Protection Agency

Scientific Investigations Report 2012-5008 


\section{Contents}

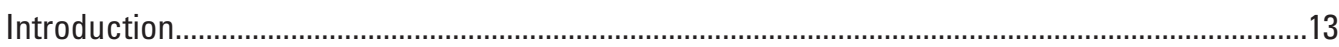

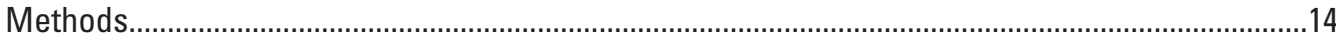

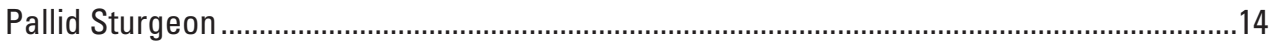

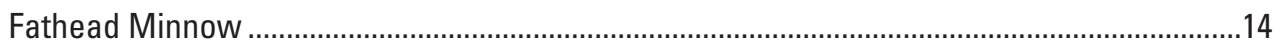

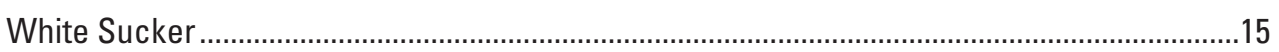

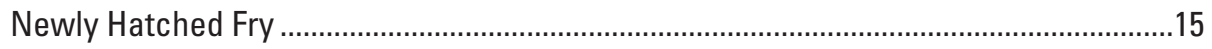

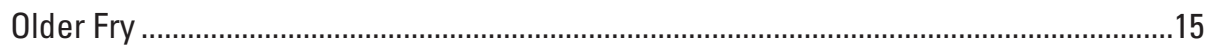

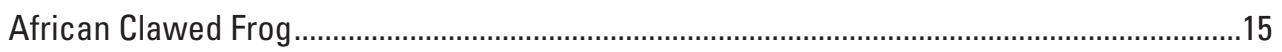

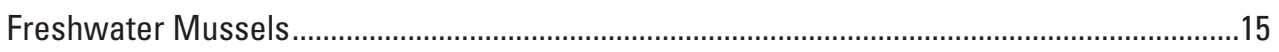

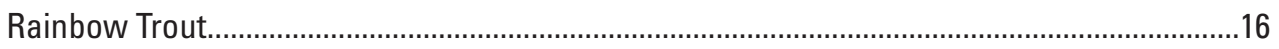

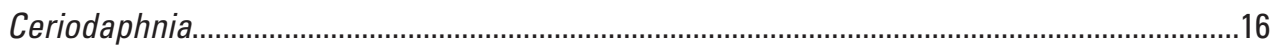

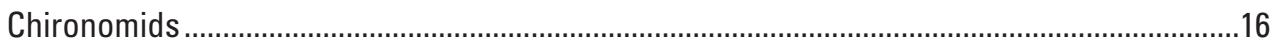

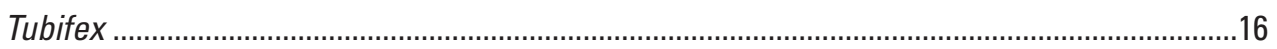

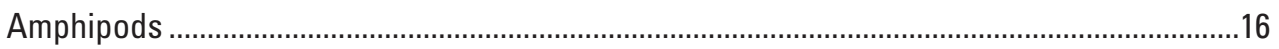

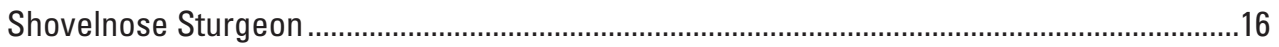

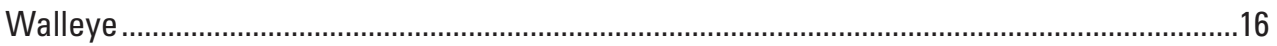

Northern Pike...........................................................................................................

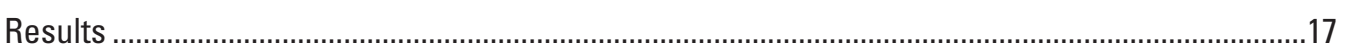

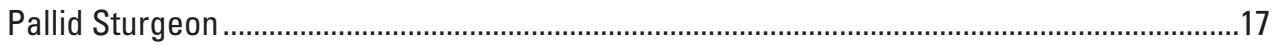

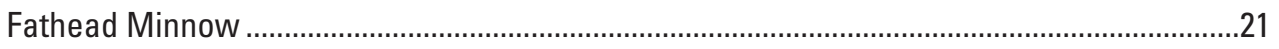

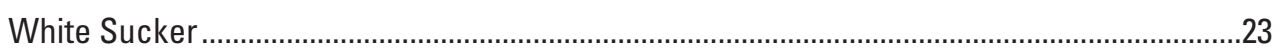

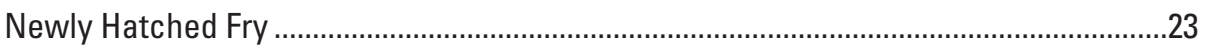

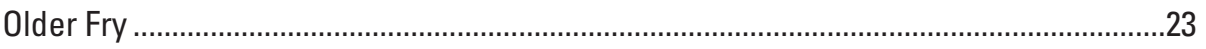

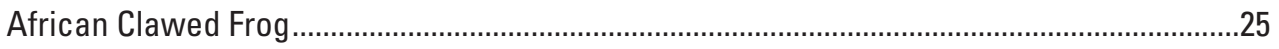

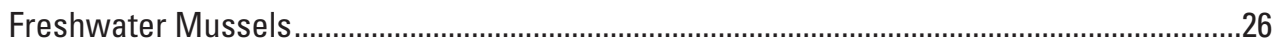

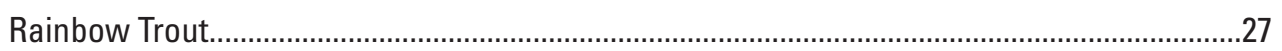

Ceriodaphnia

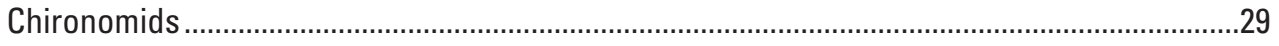

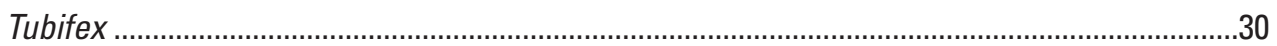

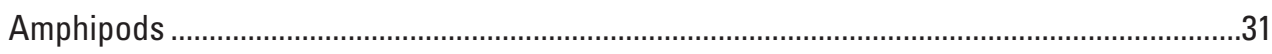

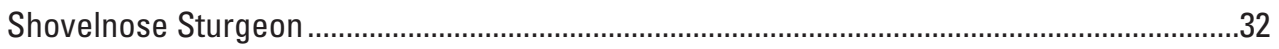

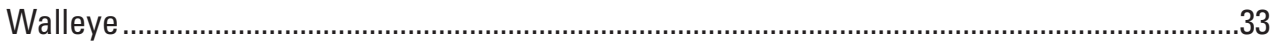

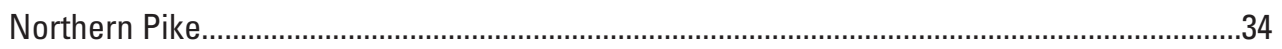

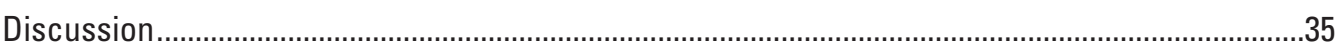

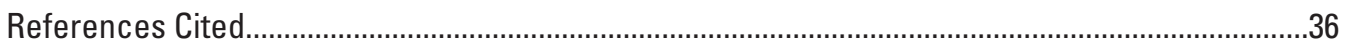

\section{Table}

2-1. Median 50-percent lethal concentrations of sodium bicarbonate for species used in experiments in the laboratory with simulated water from the Tongue and Powder Rivers, and water collected from the Yellowstone River.

2-2. Median 50-percent lethal concentrations of alkalinity and bicarbonate for species used in 96-hour experiments with water reconstituted in the laboratory to simulate water from the Tongue, Powder and Yellowstone Rivers. 
2-3. Water quality measured during acute 96 -hour exposures to sodium bicarbonate. Dilution waters were reconstituted to simulate the Tongue River, Powder River, or YellowstoneRiver

2-4. Exposure concentrations of sodium bicarbonate during a 96-hour acute toxicity experiment with 4-day-post-hatch pallid sturgeon (Scaphirhynchus albus)

2-5. Number alive and percent survival of 4-day-post-hatch pallid sturgeon (Scaphirhynchus albus) exposed to sodium bicarbonate in a 96-hour acute toxicity experiment completed in reconstituted Powder River water.

2-6. Water chemistry measured during a 96-hour acute toxicity experiment with 4-daypost-hatch pallid sturgeon (Scaphirhynchus albus).

2-7. Number alive and percent survival of 4-day-post-hatch pallid sturgeon (Scaphirhynchus albus) exposed to sodium bicarbonate in a 96-hour acute toxicity experiment completed in reconstituted Tongue River water.

2-8. Water chemistry measured during a 96-hour acute toxicity experiment with 4-daypost-hatch fathead minnows (Pimephales promelas)

2-9. Number alive and percent survival of 4-day-post-hatch fathead minnows (Pimephales promelas) exposed to sodium bicarbonate in a 96-hour acute toxicity experiment completed in reconstituted Powder River water.

2-10. Water chemistry measured during a 96-hour acute toxicity experiment with 2-day-posthatch fathead minnows (Pimephales promelas).

2-11. Number alive and percent survival of 4-day-post-hatch fathead minnows (Pimephales promelas) exposed to sodium bicarbonate in a 96-hour acute toxicity experiment completed in reconstituted Tongue River water.

2-12. Water chemistry measured during a 96-hour acute toxicity experiment with 22-daypost-hatch white sucker (Catostomus commersoni).

2-13. Number alive and percent survival of 22-day-post-hatch white suckers (Catostomus commersoni) exposed to sodium bicarbonate in a 96-hour acute toxicity experiment completed in reconstituted Tongue River water

2-14. Water chemistry measured during a 96-hour acute toxicity experiment with 22-daypost-hatch white sucker (Catostomus commersoni).

2-15. Number alive and percent survival of 22-day-post-hatch white suckers (Catostomus commersoni) exposed to sodium bicarbonate in a 96-hour acute toxicity experiment completed in reconstituted Powder River water

2-16. Water chemistry measured during a 96-hour acute toxicity experiment with less than 24-hour-post-fertilization African clawed frog (Xenopus laevis).

2-17. Number alive and percent survival of African clawed frog (Xenopus laevis) in a 96-hour acute toxicity experiment completed in reconstituted Tongue River water.. . .25

2-18. Water chemistry measured during a 96-hour acute toxicity experiment with newly transformed fatmucket mussels (Lampsilis siliquoidea).

2-19. Number alive and percent survival of newly transformed fatmucket mussels (Lampsilis siliquoidea) in a 96-hour acute toxicity experiment completed in reconstituted Tongue River water.

2-20. Water chemistry measured during a 96-hour acute toxicity experiment with experiment with rainbow trout (Oncorhynchus mykiss) completed in reconstituted Tongue River water

2-21. Number alive and percent survival of juvenile rainbow trout (Oncorhynchus mykiss) exposed to sodium bicarbonate in a 96-hour acute toxicity experiment completed in reconstituted Powder River water...... 
2-22. Water chemistry measured during a 96-hour acute toxicity experiment with adult (Ceriodaphnia dubia) completed in moderately hard reconstituted water, reconstituted Tongue River water, and reconstituted Powder River water

2-23. Number of live organisms at 0, 24, and 48 hours and percent survival at 48 hours for adult Ceriodaphnia dubia completed in moderately hard reconstituted water, reconstituted Tongue River water, and reconstituted Powder River water

2-24. Water chemistry measured in concentrated stock solutions and reconstituted (control) Tongue and Powder River waters used to make exposure concentrations for a 48-hour acute toxicity experiments with Chironomid larvae (Chironomus dilutus)

2-25. Number alive and percent survival of Chironomid larvae (Chironomus dilutus) in a 48-hour acute toxicity experiment completed in reconstituted (control) Tongue and Powder River waters.

2-26. Water chemistry measured in concentrated stock solutions of 5,000 milligrams sodium bicarbonate per liter and reconstituted (control) Tongue and Powder River waters used to make exposure concentrations for 96-hour acute toxicity experiments with Tubifex worms (Tubifex tubifex).

2-27. Number alive and percent survival (\% in parentheses) of Tubifex worms (Tubifex tubi$f e x$ ) in 96-hour acute toxicity experiments completed in reconstituted (control) Tongue and Powder River waters

2-28. Water chemistry measured in concentrated stock solutions of sodium bicarbonate and reconstituted (control) Tongue and Powder River waters used to make exposure concentrations (also presented) for 96-hour acute toxicity experiments with adult amphipods (Hyalella azteca).

2-29. Number alive and percent survival of adult amphipods (Hyalella azteca) in 96-hour acute toxicity experiments completed in reconstituted (control) Tongue and Powder River waters.

2-30. Water chemistry measured during a 96-hour acute toxicity experiment with shovelnose sturgeon (Scaphirhynchus platorynchus) completed in Yellowstone River water (control)

2-31. Number alive and percent survival of 1-day-post-hatch shovelnose sturgeon (Scaphirhynchus platorynchus) completed in Yellowstone River water (control) ..........32

2-32. Water chemistry measured during a 96-hour acute toxicity experiment with 1-day-post-hatch walleye (Sander vitreus) completed in Yellowstone River water (control).

2-33. Number alive and percent survival of 1-day-post-hatch walleye (Sander vitreus) completed in Yellowstone River water (control)

2-34. Water chemistry measured during a 96-hour acute toxicity experiment with 2-daypost-hatch northern pike (Esox lucius) completed in Yellowstone River water .34

2-35. Number alive and percent survival of 2-day-post-hatch northern pike (Esox lucius) completed in Yellowstone River water (control) 


\title{
The Acute Toxicity of Sodium Bicarbonate Defined Under Laboratory Conditions
}

\author{
By David D. Harper ${ }^{1}$, Aïda M. Farag ${ }^{1}$, Don Skaar ${ }^{2}$, and Trevor Selch ${ }^{2}$
}

\section{Introduction}

Sodium bicarbonate $\left(\mathrm{NaHCO}_{3}\right)$ is a major constituent of water produced during coalbed natural gas (CBNG) extraction in the Powder River Basin (ALL Consulting, 2003). In small ephemeral tributary streams, CBNG produced water may be a substantial part or all of the streamflow during late summer or drought periods. Little is known about the effects of $\mathrm{NaHCO}_{3}$ on aquatic organisms, and acute toxicity from exposure to $\mathrm{NaHCO}_{3}$ has not been investigated extensively. Mount and Gulley (1992) developed a model to predict the relation between salinity and toxicity in freshwater that evaluated the toxicity of a series of salts in freshwater. Mount and others (1997) completed the most diverse series of experiments to describe the toxicity of various salts and salt mixtures to assess the effects of salt exposure associated with oil field discharge; however, these experiments were limited to two species of daphnia, Ceriodaphnia dubia and Daphnia magna and one fish species, fathead minnows (Pimephales promelas). Compared to other compounds, relatively large concentrations of $\mathrm{NaHCO}_{3}$ are needed to induce acute toxicity (Dwyer and others, 2005), but among salts found in oilfield produced waters, $\mathrm{NaHCO}_{3}$ had some of the smallest lethal concentrations for fathead minnows and Ceriodaphnia (Mount and others, 1997).

The toxicity of salts to aquatic organisms can be variable (Weber-Scannell and Duffy, 2007). Some fish, invertebrates and plant species have adapted to seasonal or periodic changes in salt concentrations because of evaporation or have evolved from marine environments and are more tolerant of salinity and high alkalinity compared to other freshwater biota (Wurts, 1998; Nielsen and others, 2003). Therefore, it is critical that a wide range of aquatic organisms be used to assess the toxicity of $\mathrm{NaHCO}_{3}$. A dataset that includes multiple species is recommended for the establishment of water-quality criteria derived to protect 95 percent of species present (Stephan and others, 1985), and the dataset would provide information about the range of potential toxicity of $\mathrm{NaHCO}_{3}$ to aquatic organisms as well.

${ }^{1}$ USGS, Columbia Environmental Research Center, Jackson Field Research Station, Jackson, Wyoming.

${ }^{2}$ Montana Fish, Wildlife, and Parks, Helena, Montana.
One objective of the present study was to define the toxicity of $\mathrm{NaHCO}_{3}$ to multiple species of aquatic organisms. Then, regulatory entities could use information from the most sensitive species to calculate an acute water-quality criteria if one is desired (see chapter 6 of this report). To accomplish this objective, acute toxicity experiments were completed in the laboratory with a suite of organisms, including 7 species of fish, 5 species of invertebrates, and 1 amphibian species. The acute experiments with white sucker and fathead minnow included both newly hatched and older fry to assess the effects of age on survival. Acute toxicity experiments were performed at the Montana Fish, Wildlife and Parks laboratory in Helena, Montana and the USGS Columbia Environmental Research Center (CERC), Jackson Field Research Station, Jackson, Wyoming (hereafter referred to as Jackson Field Research Station).

Ideally, experimenting with a combination of species native to the region of concern and species that routinely are cultured in laboratories can provide a well-rounded assessment during toxicological investigations. Using species associated with the region of concern provides region-specific sensitivity information, whereas the routinely cultured species often defined as disease free, can serve as surrogate species, and the data obtained can be compared to data generated in other laboratories throughout the country or internationally. In the present study, the white sucker (Catostomus commersoni), pallid sturgeon (Scaphirhynchus albus), Shovelnose sturgeon (Scaphirhynchus platorynchus), fathead minnows (Pimephales promelas), chironomids (Chironomus dilutus), tubifex worms (Tubifex tubifex), amphipods (Hyalella azteca), freshwater mussels (fatmucket, Lampsilis siliquoidea), and Ceriodaphnia dubia species were used and are all native to the Tongue and Powder River Basins; most of the species were available commercially or from educational, State, or Federal facilities. The Northern Leopard frog (Rana pipiens), an amphibian native to the Powder and Tongue River Basins was unavailable because of logistical problems with obtaining eggs and adults at the proper lifestage for experiments, and the lack of an approved disease-free source of experimental animals. For these reasons, the African Clawed frog (Xenopus laevis) was selected as a surrogate species. Walleye (Sander vitreus), northern pike (Esox lucius), and rainbow trout (Oncorhynchus mykiss) also were used. Although these species are not native to the area of 
concern, they are popular introduced sport fish species present in the Tongue and Powder River Basins. Additionally, various lifestages of disease-free walleye, northern pike, and rainbow trout are readily available from various sources and frequently are used as experimental organisms with established test protocols.

Acute toxicity often is defined as the concentration of a chemical or substance that is lethal to 50 percent of the test population (median lethal concentration or LC50) within a specified time period, or as an effect concentration resulting in severe, measurable effects such as immobilization or developmental abnormalities (EC50; Sprague, 1973). Acute toxicity to aquatic organisms typically occurs within less than 96 hours (Rand, 1995). Typically, after 96 hours (hereafter stated as 96-h) of exposure, the most sensitive organisms will have experienced acute toxic effects, and no significant increases in mortality occur with increased exposure time (Rand, 1995). Organisms generally are exposed for 96-h in acute aquatic toxicity experiments. Small organisms with a short lifespan, such as Ceriodaphnia spp., are typically exposed for 48-h.

The acute toxicity to aquatic organisms is most efficiently determined in acute toxicity experiments that use the most sensitive species during their most sensitive lifestages (Rand, 1995). For many organisms, the early life stages are the most sensitive to most toxicants (U.S. Environmental Protection Agency, 2002); however, research into the effects of age within lifestages is limited. For this reason, a second research goal was to determine the effects of age on the toxicity of $\mathrm{NaHCO}_{3}$ to early lifestage fish and other aquatic organisms.

\section{Methods}

The experiments were performed in reconstituted waters that simulated the Tongue River or Powder River or both, except for shovelnose sturgeon, walleye and northern pike, which were exposed to water collected from the Yellowstone River near Miles City, Montana, not shown. Reconstituted waters were prepared using metal-grade salts. Reconstituted waters were prepared by adding salts to well water from the Jackson Field Research Station, not shown. Well water was used to minimize the volume of salts necessary to mimic the Tongue and Powder Rivers during low-flow conditions. Reconstituted waters were mixed in 18L HDPE carboys, and were measured for conductivity, dissolved oxygen (DO), and temperature with YSI $85^{\text {TM }}$ handheld meters (Yellow Springs Industries, Yellow Springs, Ohio). Exposure waters, including control waters, were sampled for cations and anions by the Montana Department of Health laboratory in Helena, Montana. All samples were filtered and refrigerated, and cation samples were acidified to $\mathrm{pH}-2$ with nitric acid $\left(\mathrm{HNO}_{3}^{-}\right)$.

A series of increasing amounts of $\mathrm{NaHCO}_{3}$ were added to the reconstituted waters to obtain multiple exposure concentrations for all experiments. Unless otherwise noted, experiments to determine LC50s and EC50s were completed in accordance with procedures described in the protocol outlined by the U.S. Environmental Protection Agency (2002), test organisms were not fed during exposures, and the photo period was approximately 16-h daylight and 8-h darkness/day. All acute experiments lasted 96-h, except for C. dubia and Chironomid exposures, which were 48 -h. Conductivity, DO, and temperature were measured at least once per day during all experiments with YSI $85^{\mathrm{TM}}$ handheld meters.

The Toxicity Relationship Analysis Program (TRAP; U.S. Environmental Protection Agency, 2010) was used to calculate LC50s and EC50s. Mean measured $\mathrm{NaHCO}_{3}$ concentrations were 97 percent of the nominal values; however all LC50 and EC50 calculations were completed with measured sodium bicarbonate, bicarbonate and alkalinity concentrations.

The methods used for each species are outlined in the following sections.

\section{Pallid Sturgeon}

Newly hatched pallid sturgeon were obtained from the Miles City State Fish Hatchery, Miles City, Montana, not shown, where the eggs had been incubated and hatched in Yellowstone River water. Four-days-post-hatch (dph) sturgeon were exposed to nominal concentrations of $518,864,1,440$, 2,400, and 4,000 milligrams sodium bicarbonate per liter ( $\mathrm{mg}$ $\mathrm{NaHCO}_{3} / \mathrm{L}$ ) in reconstituted Tongue or Powder River water. Three replicates of each $\mathrm{NaHCO}_{3}$ concentration were used with 10 fish per replicate.

\section{Fathead Minnow}

Newly hatched fathead minnows (FHM, Pimephales promelas) were obtained from the Jackson Field Research Station where they had been incubated, hatched, and held in water that was reconstituted to simulate Tongue River water quality. Four-days-post-hatch (4-dph) FHM were exposed to nominal concentrations of 518, 864, 1,440, 2,400, and 4,000 $\mathrm{mg} \mathrm{NaHCO}_{3} / \mathrm{L}$ in reconstituted Tongue or Powder River water. Ten fish were exposed to three replicates of each experimental concentration in the reconstituted Tongue River water and five fish per replicate were exposed in the reconstituted Powder River water. Mortality was not sufficient to calculate an LC50 for FHM exposed in the reconstituted Tongue River water quality with only 23 percent mortality occurring after 96-h of exposure to the largest concentration tested $(4,000$ $\mathrm{mg} / \mathrm{L})$. Thus, the experiment was repeated with 2-dph FHM fry. The 2-dph fry were exposed to nominal $\mathrm{NaHCO}_{3}$ concentrations of 500, 800, 1,100, 1400, 2,000, and 3,000 mg $\mathrm{NaHCO}_{3} / \mathrm{L}$ in reconstituted Tongue River water and a control treatment of reconstituted water with no $\mathrm{NaHCO}_{3}$ added. 


\section{White Sucker}

\section{Newly Hatched Fry}

White sucker fry were obtained from the Jackson Field Research Station where the eggs had been incubated and hatched and the fry were held in water reconstituted to simulate Tongue River water quality. After the white sucker fry began to feed exogenously, they were transferred from Jackson, Wyoming to Helena, Montana, not shown, where they were held in water of a similar quality. Then, 22-dph fry were exposed to nominal concentrations of $864,1,440,2,400$, 4,000 and, 6,666 $\mathrm{mg} \mathrm{NaHCO}_{3} / \mathrm{L}$ in glass Mason jars that contained 200 milliliters $(\mathrm{mL})$ of exposure water for $96-\mathrm{h}$. The control treatment contained a nominal concentration of 267 as opposed to $175 \mathrm{mg} \mathrm{NaHCO} / \mathrm{L}$ (to simulate Tongue River conditions) or 323 as opposed to $194 \mathrm{mg} \mathrm{NaHCO} / \mathrm{L}$ (to simulate Powder River conditions). For the Tongue River experiment, 10 fish were exposed to 3 replicates of each exposure concentration. For the Powder River experiment, eight fish were exposed to two replicates of each exposure concentration. Water temperature was maintained at 20 plus or minus $( \pm) 2$ degrees Celsius $\left({ }^{\circ} \mathrm{C}\right)$ and natural lighting supplemented with fluorescent lights maintain at a light: dark photoperiod of 16:8 hour (h). The fish were exposed under static-renewal conditions for 96-h and the fish were transferred to fresh solutions after 24-h, 48-h and 72-h. The fish were fed commercial Rangen Trout Starter (Rangen Inc., Buhl, Idaho) during the 2 days before the experiment was started, and $1 \mathrm{~h}$ before they were transferred to fresh exposure solutions during the experiment.

\section{Older Fry}

Additional fish were transported from the Jackson Field Research Station to Helena, Montana to evaluate the toxicity of $\mathrm{NaHCO}_{3}$ to older fry. The experiment was started with 69-dph fish that had a mean length and weight of 19.3 millimeters $(\mathrm{mm})$ and 0.038 grams $(\mathrm{g})$, respectively. The reconstituted Tongue River water (as described in the Newly Hatched Fry section) was used to expose the fish to nominal concentrations of 4,000 and 6,666 $\mathrm{mg} \mathrm{NaHCO}_{3} / \mathrm{L}$. Ten fish were exposed in each of two replicates of each concentration in a 96-h static renewal experiment. The fish were transferred to fresh experimental solutions after 24, 48 and $72 \mathrm{~h}$. The fish were fed commercial Rangen Trout Starter during the 2 days before the experiment was started and $1 \mathrm{~h}$ before they were transferred to fresh solutions during the experiment.

\section{African Clawed Frog}

Acute toxicity experiments with African clawed frogs were performed in accordance with the guidelines outlined in the standard guide for Conducting Frog Embryo Teratogenesis Assay-Xenopus (FETAX; American Society for Testing and Materials International, 1998). Adult African clawed frogs were obtained from Xenopus 1, Dexter, Michigan, USA. Adults were held in well water from the Jackson Field Research Station in $100 \mathrm{~L}$ glass aquaria maintained at $23^{\circ} \mathrm{C}$ $\left( \pm 3^{\circ} \mathrm{C}\right)$. The frogs were fed beef liver to satiation, 2 to 3 times per day, 5 days/week, and held under a light:dark photoperiod of $11: 13 \mathrm{~h}$. On the day before egg collection, adult frogs were injected with human chorionic gonadotropin at approximately $1800 \mathrm{~h}$ and pairs of male and female were placed together in darkened 4-L covered plastic food-grade containers to initiate amphiplexus, fertilization and egg deposition. Eggs were deposited on a 4-mm mesh high density polyethylene (HDPE) screen that allowed fertilized eggs to pass through the mesh into a water bath containing reconstituted Tongue River water. Fertilized eggs were collected the following morning between $0900 \mathrm{~h}$ and $1200 \mathrm{~h}$, sorted by developmental stage (Bantle and others, 1991), and 25 embryos were randomly placed in 10 - $\mathrm{mL}$ glass Petri dishes that contained $5 \mathrm{~mL}$ exposure waters, with 2 replicates per exposure. Following American Society of Testing and Materials International guidelines, 3 replicate experiments were completed.

The embryos were exposed to $750,1,000,1,500,2,500$, 3,500 , and $4,500 \mathrm{mg} \mathrm{NaHCO}_{3} / \mathrm{L}$ and a control of reconstituted Tongue River water. The embryos were exposed in a 96-h static-renewal acute toxicity experiment and the exposure water was renewed every $24 \mathrm{~h}$. Embryo mortality at 96-h and embryonic abnormalities were observed and recorded during the experiments as described in Bantle and others (1991).

\section{Freshwater Mussels}

Fatmucket mussels are native to the Tongue River drainage in both Wyoming and Montana (A.M. Cvancara, written commun., 2004; Montana Fish Wildlife and Parks, 2010). Newly transformed juvenile fatmucket mussels were obtained from Missouri State University, Springfield, Missouri. Juvenile mussels were held in a water bath in well water from the Jackson Field Research Station maintained at $20 \pm$ $2^{\circ} \mathrm{C}$. Mussels were fed a mixture of commercially prepared algae and shellfish diet during a 48-h acclimation period before the initiation of the exposure. Acute static-renewal toxicity experiments with mussels exposed to $\mathrm{NaHCO}_{3}$ were completed according to the protocol developed by Wang and others (2007), and American Society for Testing and Materials International (2006). The mussels were exposed for 96-h to nominal concentrations of 500, 1,000, 1,500, 2,000, and $2,500 \mathrm{mg} \mathrm{NaHCO}_{3} / \mathrm{L}$, and a control treatment of reconstituted Tongue River water. Five mussels were exposed to 4 replicates of each exposure concentration in 30-mL beakers. Renewal of experimental waters was performed after $48 \mathrm{~h}$. At the end of the 96-h experiments foot movement and the lack of a visible foot movement was recorded during a 5-minute observation period for the exposure effect. 


\section{Rainbow Trout}

Juvenile rainbow trout were obtained from the U.S. Fish and Wildlife Service, Ennis National Fish Hatchery, Ennis, Montana as 2-dph sac fry. These fish were exposed to a series of concentrations that ranged from 4,000-12,000 $\mathrm{mg}$ $\mathrm{NaHCO}_{3} / \mathrm{L}$ with reconstituted Tongue River water as the control and dilution water. Three replicates of 10 fish for each concentration were each held in 4-L glass aquaria. Experiments were static renewal with daily replacement of test waters.

\section{Ceriodaphnia}

C. dubia were obtained as adults from Aquatic Biosystems Inc., Fort Collins, Colorado. Neonates produced by these adults were tested following the procedures described by the U.S. Environmental Protection Agency (2002). C. dubia were exposed to a series of concentrations that ranged from 900-3,000 mg NaHCO$/ 2$ with reconstituted moderately hard (as defined in U.S. Environmental Protection Agency, 2002), reconstituted Tongue and Powder River waters as controls and dilution waters. Ten replicates with one $C$. dubia per replicate for each exposure concentration were exposed in $30-\mathrm{mL}$ plastic cups containing $15-\mathrm{mL}$ exposure water. Experiments were static renewal with daily replacement of test waters.

\section{Chironomids}

Chironomids were obtained from the Columbia Environmental Research Center, Columbia, Missouri as egg masses and tested as 1-2 week old larvae. Experiments to determine the 48-h LC50 were completed following the protocol outlined in U.S. Environmental Protection Agency (2002). Pupae were exposed to a series of concentrations that ranged from 4,000-16,000 mg $\mathrm{NaHCO}_{3} / \mathrm{L}$ with reconstituted Tongue and Powder River waters as the control and dilution waters. Four replicates with 10 Chironomids per replicate for each exposure concentration were exposed in 1-L glass aquaria. Experiments were static renewal with daily replacement of experimental waters.

\section{Tubifex}

The oligochaete annelid, Tubifex tubifex, were obtained from rearing ponds located at the U.S. Fish and Wildlife Service, Bozeman Fisheries Technology Center, Bozeman, Montana. Experiments to determine the 96-h LC50 were completed following the protocol outlined in U.S. Environmental Protection Agency (2002). Tubifex were exposed to a series of concentrations that ranged from 1,000-5,000 $\mathrm{mg} \mathrm{NaHCO} 3 / \mathrm{L}$ with reconstituted Tongue and Powder River waters as the control and dilution waters. Four replicates of 10 Tubifex per replicate for each exposure concentration were tested in 1-L glass aquaria. Experiments were static renewal with daily replacement of experimental waters.

\section{Amphipods}

Adult amphipods were obtained from a private farm pond in Lewis and Clark County, Montana. Experiments to determine the 96-h LC50 were completed following the protocol outlined in U.S. Environmental Protection Agency (2002). Amphipods were exposed to a series of concentrations that ranged from 1,000-2,000 $\mathrm{mg} \mathrm{NaHCO}_{3} / \mathrm{L}$ with reconstituted Tongue River and Powder River waters as the control and dilution water. Four replicates of ten Amphipods per replicate for each exposure concentration were tested in 1-L glass aquaria. Experiments were static renewal with daily replacement of test waters.

\section{Shovelnose Sturgeon}

Newly hatched shovelnose sturgeon were obtained from the Miles City State Fish Hatchery where eggs had been incubated and hatched in Yellowstone River water. Experiments to determine the 96-h LC50 were completed with yolk-sac fry following the protocol outlined in U.S. Environmental Protection Agency (2002). Shovelnose sturgeon were exposed to a series of concentrations that ranged from $625-10,000 \mathrm{mg}$ $\mathrm{NaHCO}_{3} / \mathrm{L}$ with Yellowstone River water as the control and dilution water. Four replicates of 5 sturgeon per replicate for each exposure concentration were tested in 1-L glass aquaria. Experiments were static renewal with daily replacement of experimental waters.

\section{Walleye}

Newly hatched walleye were obtained from the Miles City State Fish Hatchery where the eggs had been incubated and hatched in Yellowstone River water. Experiments to determine the 96-h LC50 were completed with 1-dph fry following the protocol outlined in U.S. Environmental Protection Agency (2002). Walleye were exposed to a series of concentrations that ranged from $625-5,000 \mathrm{mg} \mathrm{NaHCO} / \mathrm{L}$ with Yellowstone River water as the control and dilution water. Four replicates of 5 walleye per replicate for each exposure concentration were tested in 1-L glass aquaria. Experiments were static renewal with daily replacement of experimental waters.

\section{Northern Pike}

Northern pike were obtained from the Miles City State Fish Hatchery where the eggs had been incubated and hatched in Yellowstone River water. Experiments to determine the 96-h LC50 were completed with 1-dph fry following the protocol outlined in U.S. Environmental Protection Agency (2002). Northern pike were exposed to a series of concentrations that 
ranged from 500-8,000 mg $\mathrm{NaHCO}_{3} / \mathrm{L}$ with Yellowstone River water as the control and dilution water. Four replicates of 10 pike per replicate for each exposure concentration were tested in 1-L glass aquaria. Experiments were static renewal with daily replacement of experimental waters.

\section{Results}

The freshwater mussel, C. dubia, pallid sturgeon, shovelnose sturgeon, and FHM were the most sensitive organisms exposed to $\mathrm{NaHCO}_{3}$, alkalinity and bicarbonate $\left(\mathrm{HCO}_{3}{ }^{-}\right)$(tables $2-1$ and 2-2). Exposure conditions (DO, $\mathrm{pH}$, and temperature) did not deviate outside of acceptable values during acute exposures and the mean and range for each experiment is presented in table $2-3$. Results by species are presented in applicable tables.

\section{Pallid Sturgeon}

The 96-h LC50s for 4-dph fish were calculated as 1,356 $\mathrm{mg} / \mathrm{L} \mathrm{NaHCO}_{3}$ with 95-percent Confidence Interval (CI) of 1,206-1,507 $\mathrm{mg} \mathrm{NaHCO}_{3} / \mathrm{L}$ for the Powder River water and 1,295 mg/L NaHCO 3 95-percent CI of 927-1,662 mg $\mathrm{NaHCO}_{3} / \mathrm{L}$ for Tongue River water. Water chemistry and survival data are presented in tables $2-4,2-5,2-6,2-7$ and 2-8.

Table 2-1. Median 50-percent lethal concentrations of sodium bicarbonate for species used in experiments in the laboratory with simulated water from the Tongue and Powder Rivers, and water collected from the Yellowstone River. Exposure duration was 96 hours for all species except Ceriodaphnia and Chironomids, which was 48 hours.

[LC50, median 50-percent lethal; $\mathrm{NaHCO}_{3}$, sodium bicarbonate; $\mathrm{mg} / \mathrm{L}$, milligrams per liter; > , concentrations greater than the expressed value; --, experiments were not conducted in the specified water]

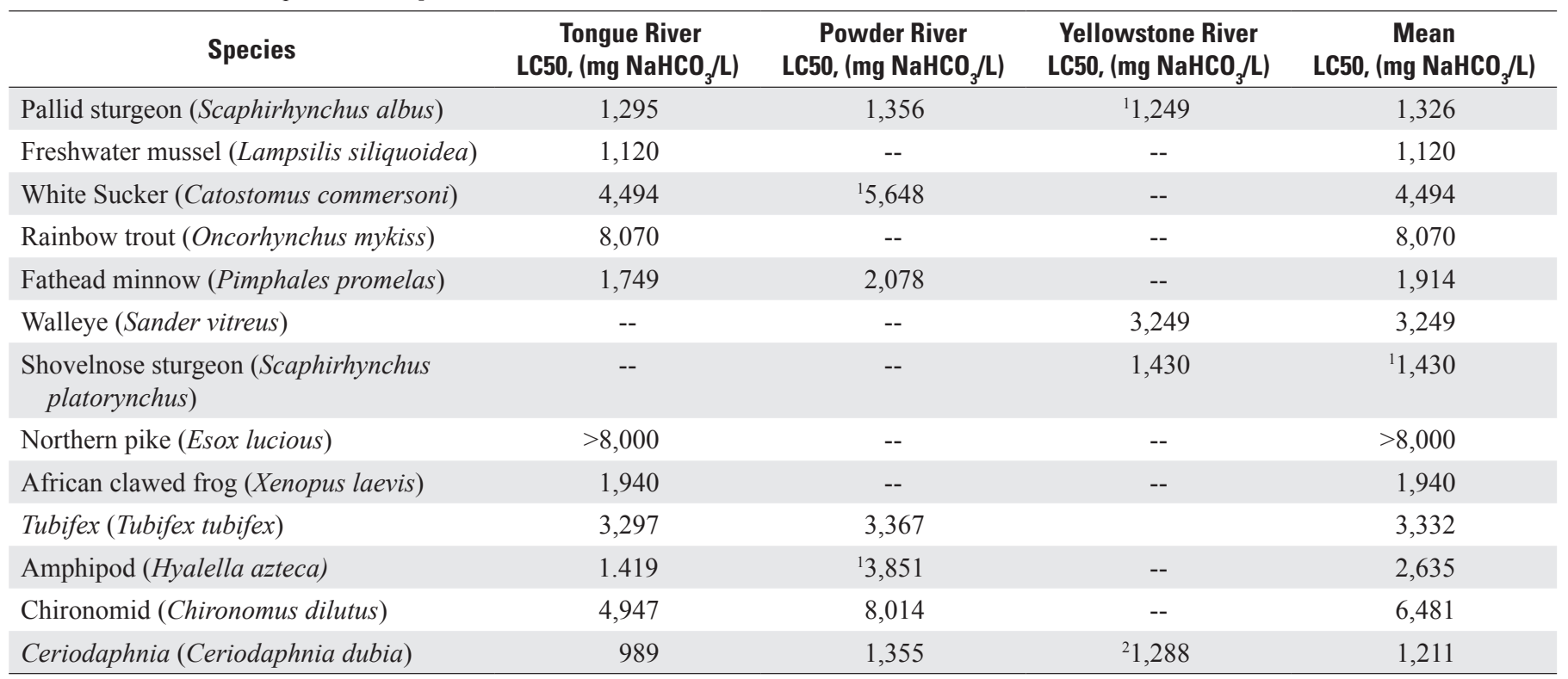

${ }^{1}$ Unreliable confidence intervals.

${ }^{2}$ Experiment completed in moderately hard reconstituted water. 
Table 2-2. Median 50-percent lethal concentrations of alkalinity and bicarbonate for species used in 96-hour experiments with water reconstituted in the laboratory to simulate water from the Tongue and Powder Rivers. Yellowstone River water was collected directly from that river for experimental use. Exposure duration for Ceriodaphnia and Chironomids was 48 hours.

[LC50, median 50-percent lethal; $\mathrm{CaCO}_{3}$, calcium carbonate; $\mathrm{mg} / \mathrm{L}$, milligrams per liter; --, experiments were not conducted in the specified water; >, concentrations greater than the expressed value]

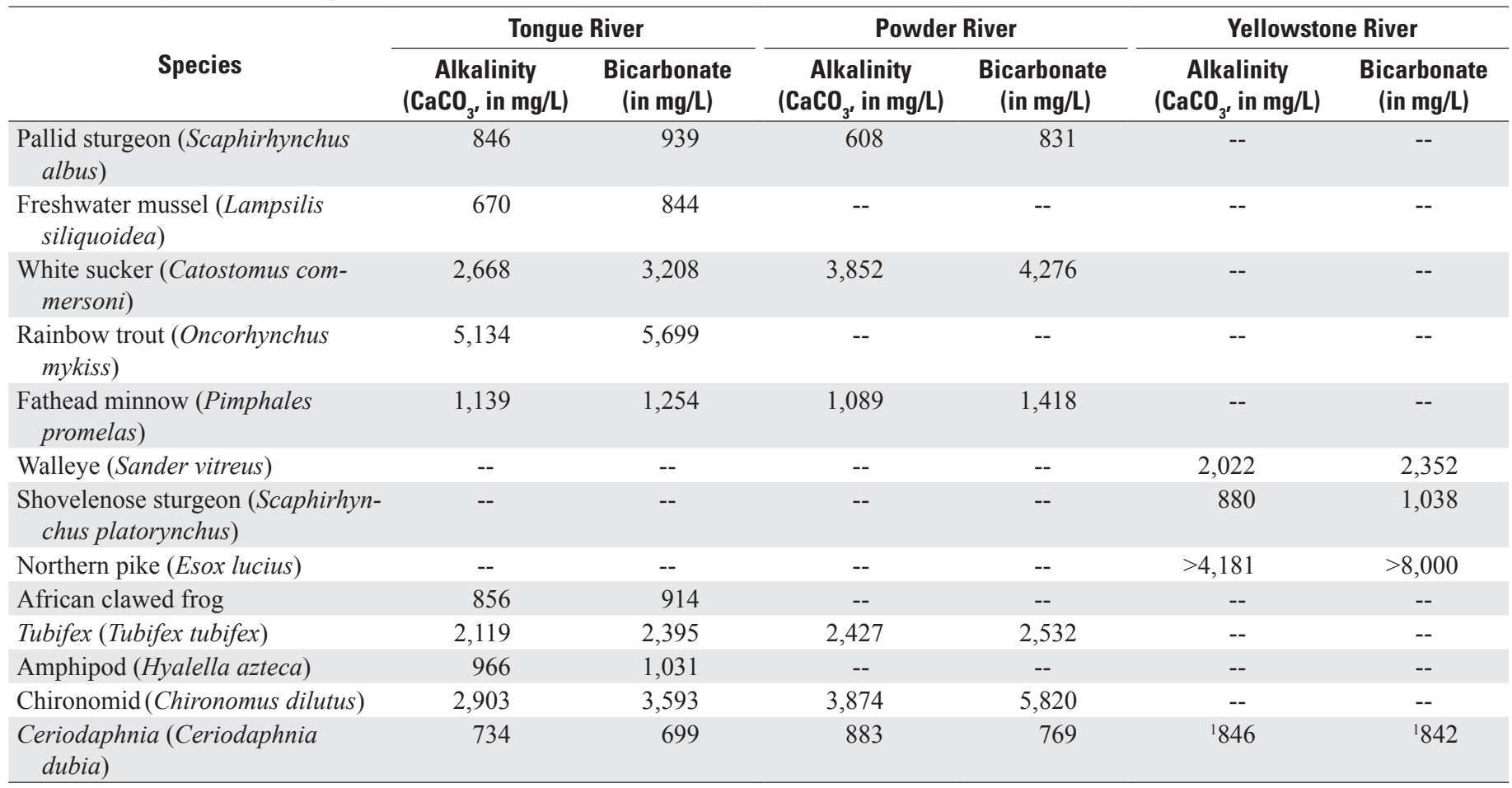

${ }^{1}$ Experiment completed in moderately hard reconstituted water.

Table 2-3. Water quality measured during acute 96-hour exposures to sodium bicarbonate. Dilution waters were reconstituted to simulate the Tongue and Powder Rivers. Yellowstone River water was collected directly from that river for experimental use. Exposure duration for Ceriodaphnia and Chironomids was 48 hours.

[mg/L, milligrams per liter; DO, dissolved oxygen; ${ }^{\circ} \mathrm{C}$, degrees Celcius $]$

\begin{tabular}{|c|c|c|c|c|c|}
\hline Species & Dilution water & $\begin{array}{c}\text { Exposure range } \\
(\mathrm{mg} / \mathrm{L})\end{array}$ & $\begin{array}{l}\text { DO range } \\
\text { (mg/L) }\end{array}$ & pH range & Temperature range $\left({ }^{\circ} \mathrm{C}\right)$ \\
\hline \multirow[t]{2}{*}{ Fathead minnow (Pimephales promelas) } & Tongue River & $500-3,000$ & $6.2-7.5$ & $8.2-8.8$ & $23-26$ \\
\hline & Powder River & $518-4,000$ & $6.3-8.5$ & $8.05-8.69$ & $24-26$ \\
\hline \multirow[t]{2}{*}{ White sucker (Catostomus commersoni) } & Tongue River & $864-6,666$ & $4.2-8.9$ & $7.97-8.74$ & $16-17$ \\
\hline & Powder River & $864-6,666$ & $6.2-8.7$ & $8.06-8.68$ & $16-17$ \\
\hline Rainbow trout (Oncorhynchus mykiss) & Tongue River & $4,000-12,000$ & $5.6-7.7$ & $7.93-8.38$ & $10-11$ \\
\hline Pallid sturgeon (Scaphirhynchus albus) & Powder River & $518-4,000$ & $4.3-7.7$ & $8.06-8.76$ & 19 \\
\hline Walleye (Sander vitreus) & Yellowstone River & $625-5,000$ & $7.9-8.9$ & $8.08-9.19$ & $14-15$ \\
\hline $\begin{array}{l}\text { Shovelnose sturgeon (Scaphirhynchus plato- } \\
\text { rynchus) }\end{array}$ & Yellowstone River & $625-10,000$ & $6.6-7.4$ & $7.79-9.28$ & $15-17$ \\
\hline Northern Pike (Esox lucius) & Yellowstone River & $500-8,000$ & $7.8-9.6$ & $7.78-9.04$ & $10-11$ \\
\hline Freshwater mussel (Lampsilis siliquoidea) & Tongue River & $500-2,500$ & 7.2 & $8.3-9.2$ & $19-22$ \\
\hline \multirow[t]{2}{*}{ Amphipod (Hyalella azteca) } & Tongue River & $1,000-2,000$ & $6.6-7.3$ & $8.6-8.8$ & 23 \\
\hline & Powder River & $1,000-2,000$ & $6.5-7.2$ & $8.1-9.0$ & 23 \\
\hline \multirow[t]{2}{*}{ Chironomid(Chironomus dilutus) } & Tongue River & $4,000-16,000$ & $5.1-6.5$ & $8.1-9.5$ & $21-23$ \\
\hline & Powder River & $4,000-16,000$ & $5.1-6.8$ & $8.1-9.3$ & $21-23$ \\
\hline \multirow[t]{2}{*}{ Ceriodaphnia (Ceriodaphnia dubia) } & Tongue River & $900-3,000$ & $7.3-7.6$ & $8.4-9.2$ & $24-27$ \\
\hline & Powder River & $900-3,000$ & $7.5-7.7$ & $8.1-9.3$ & $24-27$ \\
\hline
\end{tabular}


Table 2-4. Exposure concentrations of sodium bicarbonate during a 96-hour acute toxicity experiment with 4-day-post-hatch pallid sturgeon (Scaphirhynchus albus). Experiments were completed in reconstituted Powder River water. Exposures presented are nominal concentrations of sodium bicarbonate.

$\left[\mathrm{NaHCO}_{3}\right.$, sodium bicarbonate; $\mathrm{mg} / \mathrm{L}$, milligrams per liter; $\mathrm{CaCO}_{3}$, calcium carbonate; $\mathrm{Ca}^{2+}$, calcium, $\mathrm{Mg}^{2+}$, magnesium; $\mathrm{Na}+$, sodium; $\mathrm{K}$, potassium, $\mathrm{Cl}-$, chloride; $\mathrm{SO}_{4}^{2-}$, sulfate; ( ), range in parentheses; --, one sample was measured, no range provided. Concentrations are means unless no range is provided]

\begin{tabular}{|c|c|c|c|c|c|c|c|c|c|}
\hline \multirow{2}{*}{$\begin{array}{c}\text { Exposure } \\
(\mathrm{mg} \mathrm{NaHCO} / \mathrm{L})\end{array}$} & \multirow{2}{*}{$\begin{array}{c}\text { Alkalinity } \\
\left(\mathrm{CaCO}_{3^{\prime}} \text { in }\right. \\
\mathrm{mg} / \mathrm{L})\end{array}$} & \multirow{2}{*}{$\begin{array}{c}\text { Carbonate } \\
\left(\mathrm{CaCO}_{3^{\prime}} \text { in }\right. \\
\mathrm{mg} / \mathrm{L})\end{array}$} & \multirow{2}{*}{$\begin{array}{c}\text { Bicarbonate } \\
\text { (mg/L) }\end{array}$} & \multicolumn{6}{|c|}{ Ion concentration (mg/L) } \\
\hline & & & & $\mathrm{Ca}^{2+}$ & $\mathbf{M g}^{2+}$ & $\mathrm{Na}^{+}$ & K & Cl- & $\mathrm{SO}_{4}^{2-}$ \\
\hline Control & $(198-220)$ & $(5.3)$ & $(242-268)$ & -- & -- & -- & -- & -- & -- \\
\hline 518 & 305 & 5.3 & 366 & 65.0 & 87.7 & 378 & 16.8 & 98.7 & 855 \\
\hline 864 & $(444-480)$ & $(8.0-48.0)$ & $(517-556)$ & -- & -- & -- & -- & -- & -- \\
\hline \multirow[t]{2}{*}{1,440} & 747 & 20 & 887 & 54.0 & 87.1 & 533 & 16.7 & 98.9 & 853 \\
\hline & $(720-768)$ & $(0-48.0)$ & $(859-927)$ & -- & -- & -- & -- & -- & -- \\
\hline 2,400 & 1,240 & 40 & 1,470 & 39.1 & 86.4 & 742 & 16.7 & 99.3 & 851 \\
\hline
\end{tabular}

Table 2-5. Number alive and percent survival of 4-day-post-hatch pallid sturgeon (Scaphirhynchus albus) exposed to sodium bicarbonate in a 96-hour acute toxicity experiment completed in reconstituted Powder River water. Exposure concentrations presented are nominal concentrations of sodium bicarbonate.

$\left[\mathrm{NaHCO}_{3}\right.$, sodium bicarbonate; $\mathrm{mg} / \mathrm{L}$, millgrams per liter; ( ), percent survival]

\begin{tabular}{|c|c|c|c|c|c|}
\hline $\begin{array}{c}\text { Exposure } \\
\left(\mathrm{mg} \mathrm{NaHCO}_{3} / \mathrm{L}\right)\end{array}$ & 0 hour & 24 hours & 48 hours & 72 hours & 96 hours \\
\hline Control & $27(100)$ & $27(100)$ & $26(96)$ & $26(96)$ & $26(96)$ \\
\hline 864 & $20(100)$ & $20(100)$ & $20(100)$ & $20(100)$ & $20(100)$ \\
\hline 1,440 & $30(100)$ & $19(63)$ & $13(43)$ & $3(10)$ & $2(6)$ \\
\hline
\end{tabular}


Table 2-6. Water chemistry measured during a 96-hour acute toxicity experiment with 4-day-post-hatch pallid sturgeon (Scaphirhynchus albus). Experiments were completed in reconstituted Tongue River water. Exposures presented are nominal values for sodium bicarbonate.

$\left[\mathrm{NaHCO}_{3}\right.$, sodium bicarbonate; $\mathrm{mg} / \mathrm{L}$, milligrams per liter; $\mathrm{CaCO}_{3}$, calcium carbonate; $\mathrm{Ca}^{2+}$, calcium; $\mathrm{Mg}^{2+}$, magnesium; $\mathrm{Na}+$, sodium; $\mathrm{K}$, potassium; $\mathrm{Cl}-$, chloride; $\mathrm{SO}_{4}^{2-}$, sulfate; ( ), range in parentheses; --, one sample was measured, no range provided. Concentrations are means unless no range is provided]

\begin{tabular}{|c|c|c|c|c|c|c|c|c|c|}
\hline \multirow{2}{*}{$\begin{array}{c}\text { Exposure } \\
(\mathrm{mg} \mathrm{NaHCO} / \mathrm{L})\end{array}$} & \multirow{2}{*}{$\begin{array}{c}\text { Alkalinity } \\
\left(\mathrm{CaCO}_{3^{\prime}} \text { in }\right. \\
\mathrm{mg} / \mathrm{L})\end{array}$} & \multirow{2}{*}{$\begin{array}{c}\text { Carbonate } \\
\left(\mathrm{CaCO}_{3^{\prime}} \text { in }\right. \\
\mathrm{mg} / \mathrm{L})\end{array}$} & \multirow{2}{*}{$\begin{array}{c}\text { Bicarbonate } \\
\text { (mg/L) }\end{array}$} & \multicolumn{6}{|c|}{ Ion concentration (mg/L) } \\
\hline & & & & $\mathrm{Ca}^{2+}$ & $\mathbf{M g}^{2+}$ & $\mathrm{Na}^{+}$ & $\mathrm{K}$ & Cl- & $\mathrm{SO}_{4}^{2-}$ \\
\hline Control & $(168-178)$ & $(0-0)$ & $(205-217)$ & -- & -- & -- & -- & -- & -- \\
\hline 518 & 302 & 4.0 & 363 & 40.4 & 20.5 & 134 & 7.39 & 7.87 & 186 \\
\hline 864 & $(448-492)$ & $(16-24)$ & $(517-571)$ & -- & -- & -- & -- & -- & -- \\
\hline \multirow[t]{2}{*}{1,440} & 753 & 261.3 & 844 & 34.6 & 20.35 & 309 & 7.33 & 7.55 & 186 \\
\hline & $(728-796)$ & $(56-72)$ & $(656-902)$ & -- & -- & -- & -- & -- & -- \\
\hline 2,400 & 1,239 & 124 & 1,360 & 26.9 & 20.1 & 543 & 7.25 & 7.13 & 185 \\
\hline
\end{tabular}

Table 2-7. Number alive and percent survival of 4-day-post-hatch pallid sturgeon (Scaphirhynchus albus) exposed to sodium bicarbonate in a 96-hour acute toxicity experiment completed in reconstituted Tongue River water. Exposures presented are nominal values for sodium bicarbonate.

$\left[\mathrm{NaHCO}_{3}\right.$, sodium bicarbonate; $\mathrm{mg} / \mathrm{L}$, millgrams per liter; ( ), percent survival]

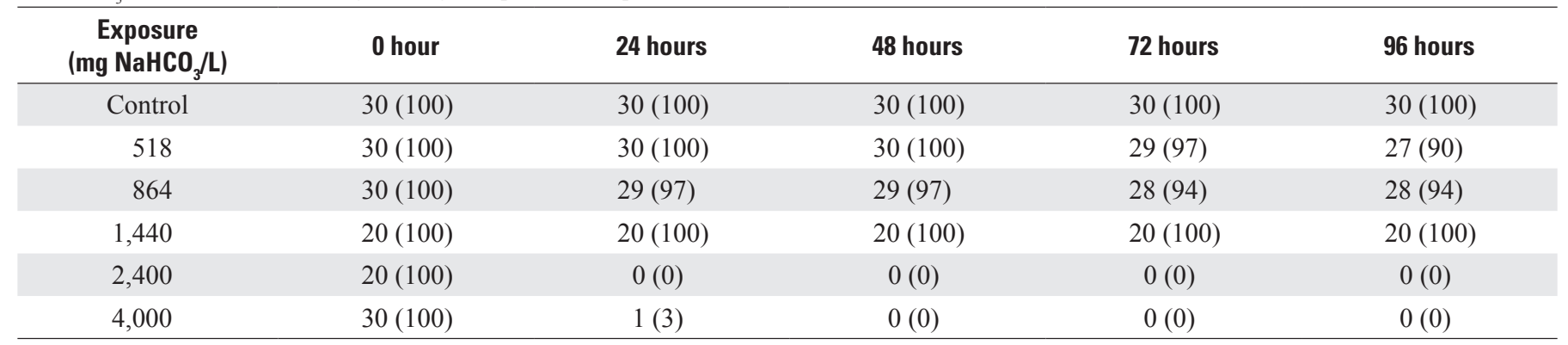




\section{Fathead Minnow}

The 96-h LC50 for 4-dph FHM exposed in reconstituted Powder River water was $2,078 \mathrm{mg} \mathrm{NaHCO}_{3} / \mathrm{L}$ with 95 -percent CI of 769-3,388 mg/L (table 2-9). With 2-dph FHM exposed in reconstituted Tongue River water, the LC50 was $1,749 \mathrm{mg}$ $\mathrm{NaHCO}_{3} / \mathrm{L}$ with 95-percent CI of 1,604-1,895 mg $\mathrm{NaHCO}_{3} / \mathrm{L}$, respectively. Water chemistry and survival data are presented in tables 2-10 and 2-11.

Table 2-8. Water chemistry measured during a 96-hour acute toxicity experiment with 4-day-post-hatch fathead minnows (Pimephales promelas). Experiments were completed in reconstituted Powder River water. Exposures presented are nominal values for sodium bicarbonate.

$\left[\mathrm{NaHCO}_{3}\right.$, sodium bicarbonate; $\mathrm{mg} / \mathrm{L}$, milligrams per liter; $\mathrm{CaCO}_{3}$, calcium carbonate; $\mathrm{Ca}^{2+}$, calcium; $\mathrm{Mg}^{2+}$, magnesium; $\mathrm{Na}+$, sodium; $\mathrm{K}$, potassium; $\mathrm{Cl}$-, chloride; $\mathrm{SO}_{4}^{2-}$, sulfate; ( ), range in parentheses; --, one sample was measured, no range provided. Concentrations are means unless no range is provided]

\begin{tabular}{|c|c|c|c|c|c|c|c|c|c|}
\hline \multirow{2}{*}{$\begin{array}{c}\text { Exposure } \\
\left(\mathrm{mg} \mathrm{NaHCO}_{3} / \mathrm{L}\right)\end{array}$} & \multirow{2}{*}{$\begin{array}{c}\text { Alkalinity } \\
\text { (CaCO }{ }_{3^{\prime}} \text { in } \\
\mathrm{mg} / \mathrm{L})\end{array}$} & \multirow{2}{*}{$\begin{array}{c}\text { Carbonate } \\
\left(\mathrm{CaCO}_{3^{\prime}} \text { in }\right. \\
\mathrm{mg} / \mathrm{L})\end{array}$} & \multirow{2}{*}{$\begin{array}{l}\text { Bicarbonate } \\
\text { (mg/L) }\end{array}$} & \multicolumn{6}{|c|}{ Ion concentration (mg/L) } \\
\hline & & & & $\mathrm{Ca}^{2+}$ & $\mathbf{M g}^{2+}$ & $\mathrm{Na}^{+}$ & K & $\mathrm{Cl}-$ & $\mathrm{SO}_{4}^{2-}$ \\
\hline Control & (196-204) & $(8-8)$ & (229-239) & -- & -- & -- & -- & -- & -- \\
\hline 518 & 320.5 & 17 & 303.5 & 76.3 & 92.0 & 332 & 12.0 & 100.4 & 923 \\
\hline \multirow[t]{2}{*}{864} & 491 & 21 & 470 & 72.8 & 91.6 & 417 & 12.4 & 99.2 & 911 \\
\hline & $(482-500)$ & $(20-24)$ & $(558-585)$ & -- & -- & -- & -- & -- & -- \\
\hline \multirow[t]{2}{*}{1,440} & 772 & 21 & 751 & 66.9 & 90.8 & 557 & 13.1 & 97.4 & 892 \\
\hline & $(762-786)$ & $(20-24)$ & $(905-929)$ & -- & -- & -- & -- & -- & -- \\
\hline 2,400 & 1,285 & 62 & 1,223 & 57.2 & 89.5 & 791 & 14.2 & 94.3 & 861 \\
\hline
\end{tabular}

Table 2-9. Number alive and percent survival of 4-day-post-hatch fathead minnows (Pimephales promelas) exposed to sodium bicarbonate in a 96-hour acute toxicity experiment completed in reconstituted Powder River water. Exposures presented are nominal values for sodium bicarbonate.

$\left[\mathrm{NaHCO}_{3}\right.$, sodium bicarbonate; mg/L, milligrams per liter; ( ), percent survival]

\begin{tabular}{|c|c|c|c|c|c|}
\hline $\begin{array}{c}\text { Exposure } \\
\text { (mg NaHCO} / \mathrm{L})\end{array}$ & 0 hour & 24 hours & 48 hours & 72 hours & 96 hours \\
\hline Control & $15(100)$ & $15(100)$ & 15 (100) & $15(100)$ & $15(100)$ \\
\hline 864 & $15(100)$ & $15(100)$ & $15(100)$ & $14(93)$ & $12(80)$ \\
\hline 1,440 & $15(100)$ & $14(93)$ & $13(87)$ & $9(60)$ & $9(60)$ \\
\hline
\end{tabular}


Table 2-10. Water chemistry measured during a 96-hour acute toxicity experiment with 2-day-post-hatch fathead minnows (Pimephales promelas). Experiments were completed in reconstituted Tongue River water. Exposures presented are nominal values for sodium bicarbonate.

$\left[\mathrm{NaHCO}_{3}\right.$, sodium bicarbonate; $\mathrm{mg} / \mathrm{L}$, milligrams per liter; $\mathrm{CaCO}_{3}$, calcium carbonate; $\mathrm{Ca}^{2+}$, calcium; $\mathrm{Mg}^{2+}$, magnesium; $\mathrm{Na}+$, sodium; $\mathrm{K}$, potassium; $\mathrm{Cl}$, chloride; $\mathrm{SO}_{4}^{2}$, sulfate; ( ), standard error in parentheses; --, one sample was measured, no range provided. Concentrations are means unless no range is provided]

\begin{tabular}{|c|c|c|c|c|c|c|c|c|c|c|}
\hline \multirow{2}{*}{$\begin{array}{c}\text { Exposure } \\
\text { (mg NaHCO} / \text { /L) }\end{array}$} & \multirow{2}{*}{$\begin{array}{c}\text { Alkalinity } \\
\text { (CaCO } \text {, in } \\
\mathrm{mg} / \mathrm{L})\end{array}$} & \multirow{2}{*}{$\begin{array}{c}\text { Carbonate } \\
\left(\mathrm{CaCO}_{3^{\prime}} \text {, in }\right. \\
\mathrm{mg} / \mathrm{L})\end{array}$} & \multirow{2}{*}{$\begin{array}{c}\text { Bicarbonate } \\
\text { (mg/L) }\end{array}$} & \multicolumn{7}{|c|}{ Ion concentration (mg/L) } \\
\hline & & & & $\mathrm{Ca}^{2+}$ & $\mathrm{Mg}^{2+}$ & $\mathrm{Na}^{+}$ & $\mathbf{K}$ & $\mathrm{Cl}-$ & $\mathrm{NO}_{3}$ & $\mathrm{SO}_{4}^{2-}$ \\
\hline Control & (11) & (6) & (18) & (2.6) & $(.35)$ & $(.78)$ & $(.03)$ & $(.2)$ & $(.01)$ & $(2.5)$ \\
\hline 500 & 374 & 15 & 438 & 47 & 34 & 134 & 2.4 & 2.3 & .3 & 142 \\
\hline \multirow[t]{2}{*}{800} & 532 & 114 & 627 & 37 & 34 & 216 & 2.4 & 2.2 & .3 & 142 \\
\hline & (13) & (102) & (27) & (3.3) & $(.6)$ & $(4.5)$ & $(.04)$ & $(.03)$ & $(.01)$ & (1.3) \\
\hline \multirow[t]{2}{*}{1,100} & 704 & 60 & 766 & 27 & 34 & 293 & 2.5 & 2.2 & .3 & 144 \\
\hline & (18) & -- & -- & -- & -- & -- & -- & -- & -- & -- \\
\hline 1,400 & 899 & 62 & 1,020 & 22 & 34 & 390 & 2.6 & 2.4 & .3 & 146 \\
\hline \multirow[t]{2}{*}{3,000} & 1,880 & 265 & 1,970 & 12 & 34 & 812 & 3.4 & 5.1 & .3 & 148 \\
\hline & (72) & (79) & (26) & (5.1) & $(.79)$ & $(24)$ & $(.54)$ & $(.4)$ & $(.01)$ & (3.2) \\
\hline
\end{tabular}

Table 2-11. Number alive and percent survival of 2-day-post-hatch fathead minnows (Pimephales promelas) exposed to sodium bicarbonate in a 96-hour acute toxicity experiment completed in reconstituted Tongue River water. Exposures presented are nominal values for sodium bicarbonate.

[ $\mathrm{NaHCO}_{3}$, sodium bicarbonate; $\mathrm{mg} / \mathrm{L}$, milligrams per liter; ( ), percent survival]

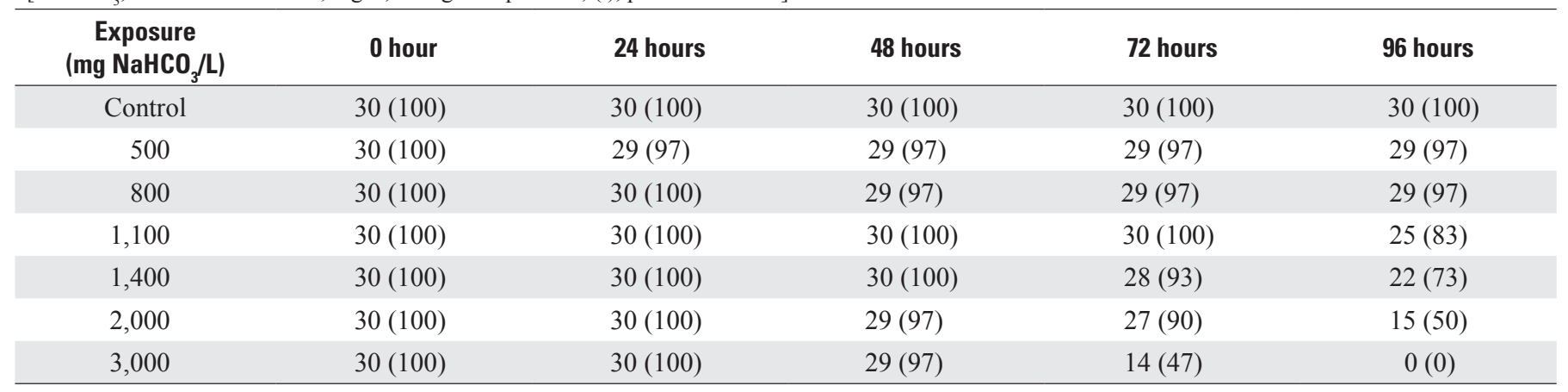




\section{White Sucker}

\section{Newly Hatched Fry}

There was greater mortality among fish exposed in the reconstituted Tongue River water compared with fish exposed in reconstituted Powder River water. The LC50 for white suckers exposed to reconstituted Tongue River water was 4,494 mg $\mathrm{NaHCO}_{3} / \mathrm{L}$ with 95-percent CI of 2,891-6,097 mg $\mathrm{NaHCO}_{3} / \mathrm{L}$. Water chemistry and survival data are presented in tables 2-12 and 2-13 In reconstituted Powder River water, the calculated 96-h LC50 was 5,648 mg NaHCO$/ 2$ L. Reliable 95-percent CI could not be calculated. Water chemistry and survival data are presented in tables 2-14 and 2-15.

\section{Older Fry}

The limited number of exposure levels did not allow the LC50 to be calculated for older white suckers, but mortality rates were 9.5 percent, 13.6 percent, and 50 percent for the control, 4,000 and 6,666 $\mathrm{mg} \mathrm{NaHCO}_{3} / \mathrm{L}$ (table 2-13.), respectively. These results suggest that the older fry were somewhat more tolerant of the $\mathrm{NaHCO}_{3}$ than the younger fish.

Table 2-12. Water chemistry measured during a 96-hour acute toxicity experiment with 22-day-post-hatch white sucker (Catostomus commersoni). Experiments were completed in reconstituted Tongue River water. Exposures presented are nominal values for sodium bicarbonate.

$\left[\mathrm{NaHCO}_{3}\right.$, sodium bicarbonate; mg/L, milligrams per liter; $\mathrm{CaCO}_{3}$, calcium carbonate; $\mathrm{Ca}^{2+}$, calcium; $\mathrm{Mg}^{2+}$, magnesium; $\mathrm{Na}+$, sodium; $\mathrm{K}$, potassium; $\mathrm{Cl}-$, chloride; $\mathrm{SO}_{4}^{2-}$, sulfate; ( ), range; --, one sample was measured, no range provided. Concentrations are means unless no range is provided]

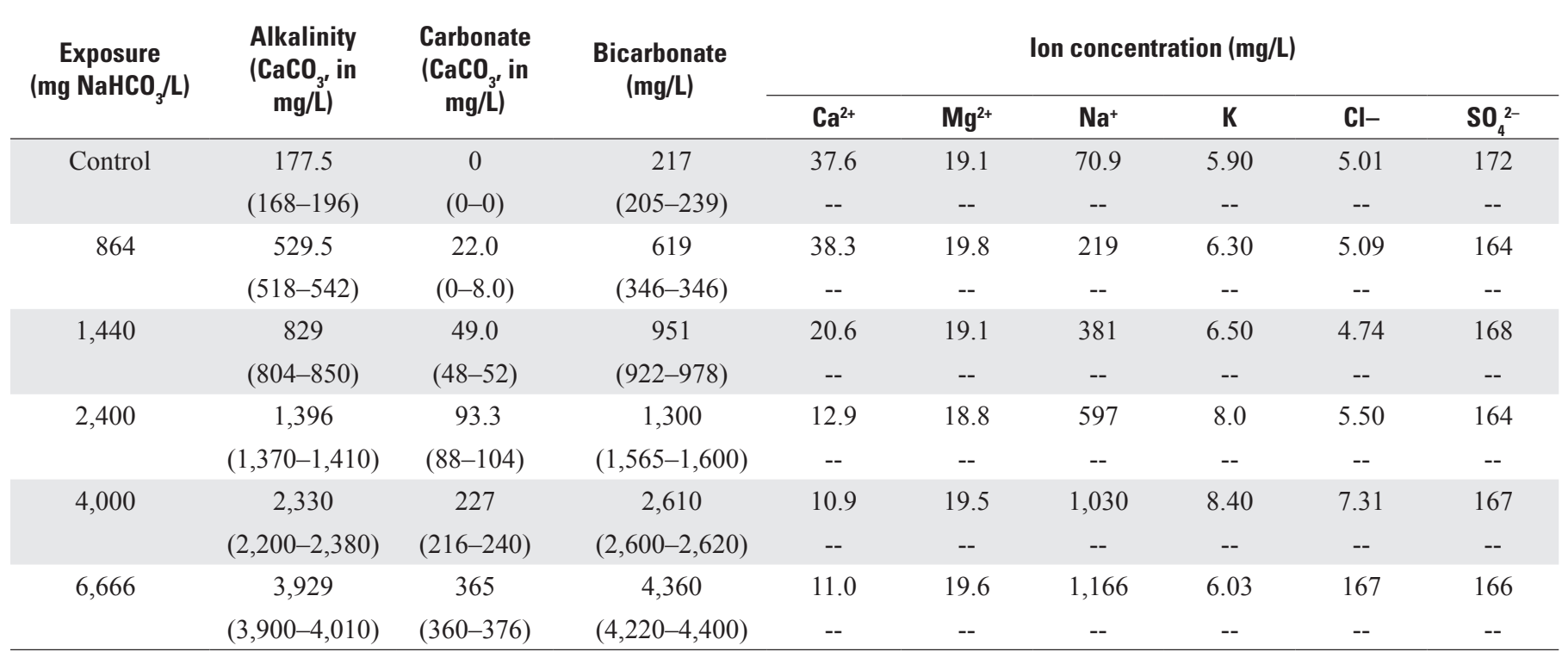

Table 2-13. Number alive and percent survival of 22-day-post-hatch white suckers (Catostomus commersoni) exposed to sodium bicarbonate in a 96-hour acute toxicity experiment completed in reconstituted Tongue River water. Exposures presented are nominal values for sodium bicarbonate.

$\left[\mathrm{NaHCO}_{3}\right.$, sodium bicarbonate; $\mathrm{mg} / \mathrm{L}$, milligrams per liter; ( ), percent survival $]$

\begin{tabular}{|c|c|c|c|c|c|}
\hline $\begin{array}{c}\text { Exposure } \\
\left(\mathrm{mg} \mathrm{NaHCO}_{3} / \mathrm{L}\right)\end{array}$ & 0 hour & 24 hours & 48 hours & 72 hours & 96 hours \\
\hline Control & $26(100)$ & $26(100)$ & $24(92)$ & $24(92)$ & $24(92)$ \\
\hline 864 & $31(100)$ & $28(90)$ & $28(90)$ & $28(90)$ & $27(87)$ \\
\hline 1,440 & $30(100)$ & $30(100)$ & $30(100)$ & $30(100)$ & $28(93)$ \\
\hline 2,400 & $30(100)$ & $28(93)$ & $28(93)$ & $27(90)$ & $26(87)$ \\
\hline 4,000 & $30(100)$ & $21(70)$ & $19(63)$ & $17(57)$ & $15(50)$ \\
\hline 6,666 & $30(100)$ & $19(63)$ & $11(37)$ & $10(30)$ & $10(30)$ \\
\hline
\end{tabular}


Table 2-14. Water chemistry measured during a 96-hour acute toxicity experiment with 22-day-post-hatch white sucker (Catostomus commersoni). Experiments were completed in reconstituted Powder River water. Exposures presented are nominal values for sodium bicarbonate.

$\left[\mathrm{NaHCO}_{3}\right.$, sodium bicarbonate; mg/L, milligrams per liter; $\mathrm{CaCO}_{3}$, calcium carbonate; $\mathrm{Ca}^{2+}$, calcium; $\mathrm{Mg}^{2+}$, magnesium; $\mathrm{Na}+$, sodium; $\mathrm{K}$, potassium; $\mathrm{Cl}-$, chloride; $\mathrm{SO}_{4}^{2-}$, sulfate; ( ), range; --, one sample was measured, no range provided. Concentrations are means unless no range is provided]

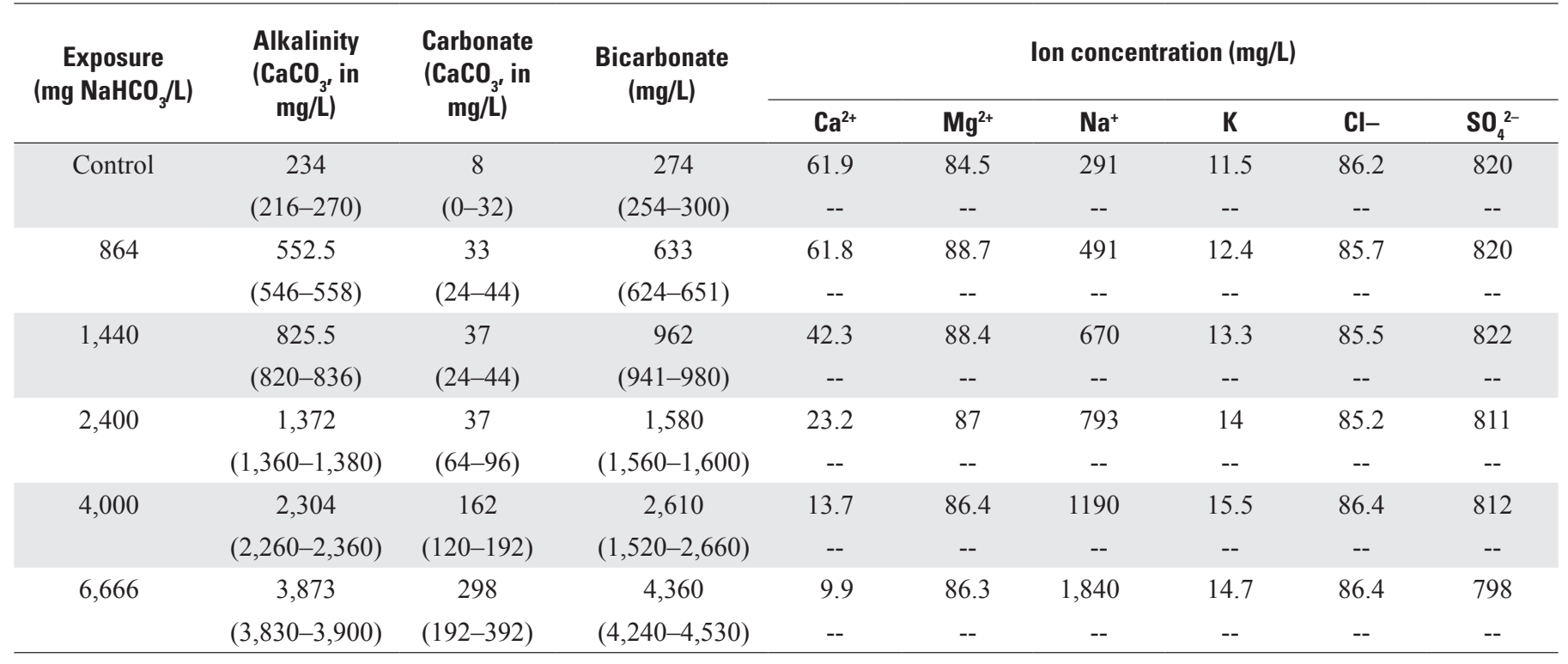

Table 2-15. Number alive and percent survival of 22-day-post-hatch white suckers (Catostomus commersoni) exposed to sodium bicarbonate in a 96-hour acute toxicity experiment completed in reconstituted Powder River water. Exposures presented are nominal values for sodium bicarbonate.

$\left[\mathrm{NaHCO}_{3}\right.$, sodium bicarbonate; $\mathrm{mg} / \mathrm{L}$, milligrams per liter; ( ), percent survival]

\begin{tabular}{|c|c|c|c|c|c|}
\hline $\begin{array}{c}\text { Exposure } \\
\left(\mathrm{mg} \mathrm{NaHCO}_{3} / \mathrm{L}\right)\end{array}$ & 0 hour & 24 hours & 48 hours & 72 hours & 96 hours \\
\hline Control & $16(100)$ & $15(94)$ & $15(94)$ & $15(94)$ & $15(94)$ \\
\hline 1,440 & $14(100)$ & $14(100)$ & $13(93)$ & $13(93)$ & $13(93)$ \\
\hline 2,400 & $16(100)$ & $16(100)$ & $16(100)$ & $16(100)$ & $15(94)$ \\
\hline
\end{tabular}




\section{African Clawed Frog}

The mean LC50 for four acute toxicity experiments with the African clawed frog was $1,940 \mathrm{mg} \mathrm{NaHCO} / \mathrm{L}$ with mean 95-percent CI of 1,482-2,890 $\mathrm{mg} \mathrm{NaHCO}_{3} / \mathrm{L}$. Non-Lethal abnormalities occurred with increasing $\mathrm{NaHCO}_{3}$ concentrations, primarily as mild stunting and mild dorsal and lateral flexure of the tail. In some individuals that ultimately were counted as mortalities, severe stunting and flexure of the tail was observed before the end of the 96-h exposure. Mild flexure of the tail was observed in some survivors after the 96-h exposure. The EC50 ranged from 955 to $1,307 \mathrm{mg} \mathrm{NaHCO} / \mathrm{L}$, with a mean of $1,108 \mathrm{mg} \mathrm{NaHCO}_{3} / \mathrm{L}$. Water chemistry and survival data are presented in tables $2-16$ and 2-17.

Table 2-16. Water chemistry measured during a 96-hour acute toxicity experiment with African clawed frog (Xenopus laevis). Experiments were completed in reconstituted Tongue River water. Exposures presented are nominal values for sodium bicarbonate. Frog embryos were less than 24-hour-post-fertilization at the start of the exposure.

$\left[\mathrm{NaHCO}_{3}\right.$, sodium bicarbonate; mg/L, milligrams per liter; $\mathrm{CaCO}_{3}$, calcium carbonate; $\mathrm{Ca}^{2+}$, calcium; $\mathrm{Mg}^{2+}$, magnesium; $\mathrm{Na}+$, sodium; $\mathrm{K}$, potassium; $\mathrm{Cl}-$, chloride; $\mathrm{NO}_{3}-$, nitrate; $\mathrm{SO}_{4}^{2-}$, sulfate; ( ), standard error in parentheses. Concentrations are means]

\begin{tabular}{|c|c|c|c|c|c|c|c|c|c|c|}
\hline \multirow{2}{*}{$\begin{array}{c}\text { Exposure } \\
\text { (mg NaHCO} / \mathrm{L})\end{array}$} & \multirow{2}{*}{$\begin{array}{c}\text { Alkalinity } \\
\left(\mathrm{CaCO}_{3^{\prime}} \text { in }\right. \\
\mathrm{mg} / \mathrm{L})\end{array}$} & \multirow{2}{*}{$\begin{array}{c}\text { Carbonate } \\
\left(\mathrm{CaCO}_{3^{\prime}} \text {, in }\right. \\
\mathrm{mg} / \mathrm{L})\end{array}$} & \multirow{2}{*}{$\begin{array}{c}\text { Bicarbonate } \\
\text { (mg/L) }\end{array}$} & \multicolumn{7}{|c|}{ Ion concentration (mg/L) } \\
\hline & & & & $\begin{array}{c}\text { Mean } \\
\mathrm{Ca}^{2+}\end{array}$ & $\begin{array}{c}\text { Mean } \\
\mathrm{Mg}^{2+}\end{array}$ & $\begin{array}{c}\text { Mean } \\
\mathrm{Na}^{+}\end{array}$ & $\begin{array}{c}\text { Mean } \\
\mathrm{K}\end{array}$ & $\begin{array}{c}\text { Mean } \\
\text { Cl- }\end{array}$ & $\mathrm{NO}_{3}^{-}$ & $\begin{array}{l}\text { Mean } \\
\mathrm{SO}_{4}^{2-}\end{array}$ \\
\hline \multirow[t]{2}{*}{ Control } & 192 & 12.3 & 220 & 45 & 33 & 45 & 2.4 & 2 & 0.3 & 139 \\
\hline & $(4.3)$ & $(1.1)$ & $(3.5)$ & $(1.6)$ & (.8) & (.3) & $(.10)$ & $(.1)$ & $(.01)$ & $(.4)$ \\
\hline 500 & 306 & 39 & 325 & 33 & 30 & 131 & 2.3 & 2.2 & .2 & 143 \\
\hline \multirow[t]{2}{*}{750} & 464 & 46 & 508 & 18 & 30 & 193 & 4 & 3.2 & .3 & 132 \\
\hline & $(6.3)$ & $(1.7)$ & $(9.8)$ & $(2.8)$ & (.4) & (1) & $(1.6)$ & $(1.3)$ & $(.01)$ & $(3.5)$ \\
\hline \multirow[t]{2}{*}{1,000} & 600 & 77 & 637 & 12 & 30 & 261 & 2.6 & 2 & .2 & 130 \\
\hline & $(8.5)$ & (11) & (22) & $(3.9)$ & (.6) & $(2.9)$ & $(.1)$ & $(.1)$ & $(.02)$ & $(3.2)$ \\
\hline 2,500 & $(10.8)$ & $(75)$ & $(37)$ & $(3.5)$ & (.3) & $(1.5)$ & (.1) & $(.05)$ & $(.01)$ & $(.4)$ \\
\hline \multirow[t]{2}{*}{3,000} & 1,877 & 265 & 1,965 & 12 & 34 & 812 & 3.4 & 5.1 & .3 & 148 \\
\hline & (72) & (79) & (26) & $(5.1)$ & $(.79)$ & (24) & $(.54)$ & (.4) & $(.01)$ & $(3.2)$ \\
\hline \multirow[t]{2}{*}{3,500} & 2,070 & 159 & 2,330 & 4.3 & 31 & 901 & 2.8 & 4.9 & .3 & 135 \\
\hline & (18) & $(5.2)$ & (19.5) & $(.5)$ & $(.2)$ & $(8.9)$ & (.1) & (.1) & $(.01)$ & $(.4)$ \\
\hline \multirow[t]{2}{*}{4,500} & 2,640 & 408 & 2,730 & 3.6 & 31 & 1,093 & 2.8 & 5.4 & .3 & 135 \\
\hline & (18) & (50) & (39) & $(1.0)$ & $(1.0)$ & $(4.8)$ & (.1) & $(.2)$ & $(.01)$ & $(.8)$ \\
\hline
\end{tabular}

Table 2-17. Number alive and percent survival of African clawed frog (Xenopus laevis) in a 96-hour acute toxicity experiment completed in reconstituted Tongue River water. Exposures presented are nominal concentrations sodium bicarbonate. Frog embryos were less than 24-hour-post-fertilization at the start of the exposure.

$\left[\mathrm{NaHCO}_{3}\right.$, sodium bicarbonate; mg/L, milligrams per liter; ( ), percent survival; --, not applicable]

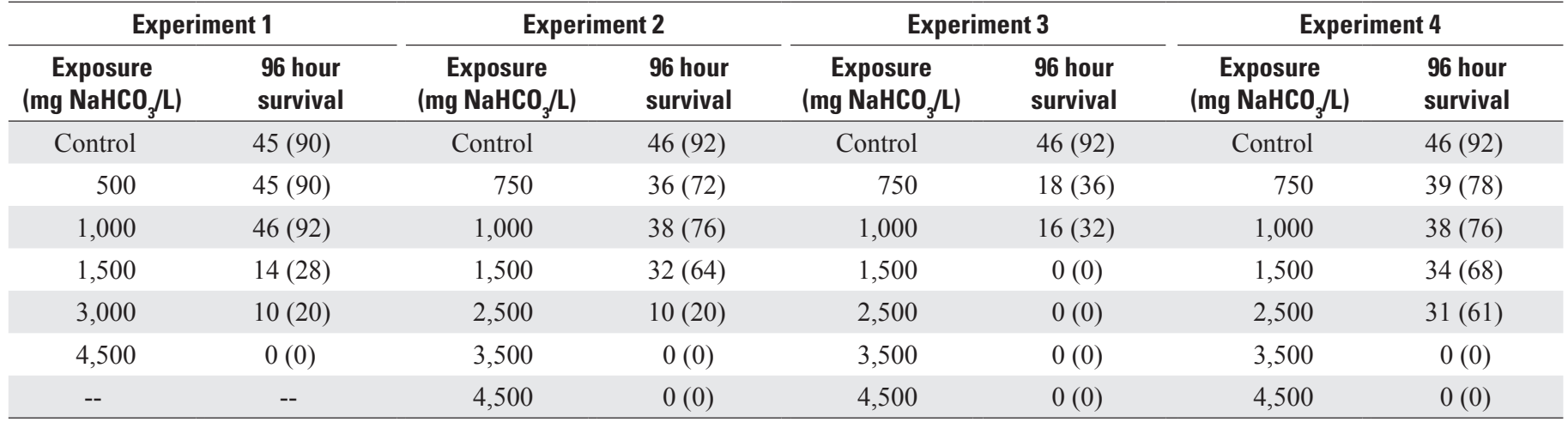




\section{Freshwater Mussels}

The EC50 for newly transformed juvenile fatmucket mussels exposed to reconstituted Tongue River water was $1,120 \mathrm{mg} \mathrm{NaHCO} / \mathrm{L}$, with 95-percent CI of 1,099-1,497 $\mathrm{mg} \mathrm{NaHCO}_{3} / \mathrm{L}$. Although the lack of foot movement was the ultimate measure of toxicity, mussels that did not display foot movement within the 5-minute observation period were all observed as mortalities, and under closer examination as empty shells at the end of the experiment. Water chemistry and survival data are presented in tables $2-18$ and 2-19.

Table 2-18. Water chemistry measured during a 96-hour acute toxicity experiment with newly transformed fatmucket mussels (Lampsilis siliquoidea). Experiments were completed in reconstituted Tongue River water. Exposures presented are nominal values for sodium bicarbonate. Sample size of two for each.

$\left[\mathrm{NaHCO}_{3}\right.$, sodium bicarbonate; $\mathrm{mg} / \mathrm{L}$, milligrams per liter; $\mathrm{CaCO}_{3}$, calcium carbonate; $\mathrm{Ca}^{2+}$, calcium; $\mathrm{Mg}^{2+}$, magnesium; $\mathrm{Na}+$, sodium; $\mathrm{K}$, potassium; $\mathrm{Cl}-$, chloride; $\mathrm{SO}_{4}^{2-}$, sulfate]

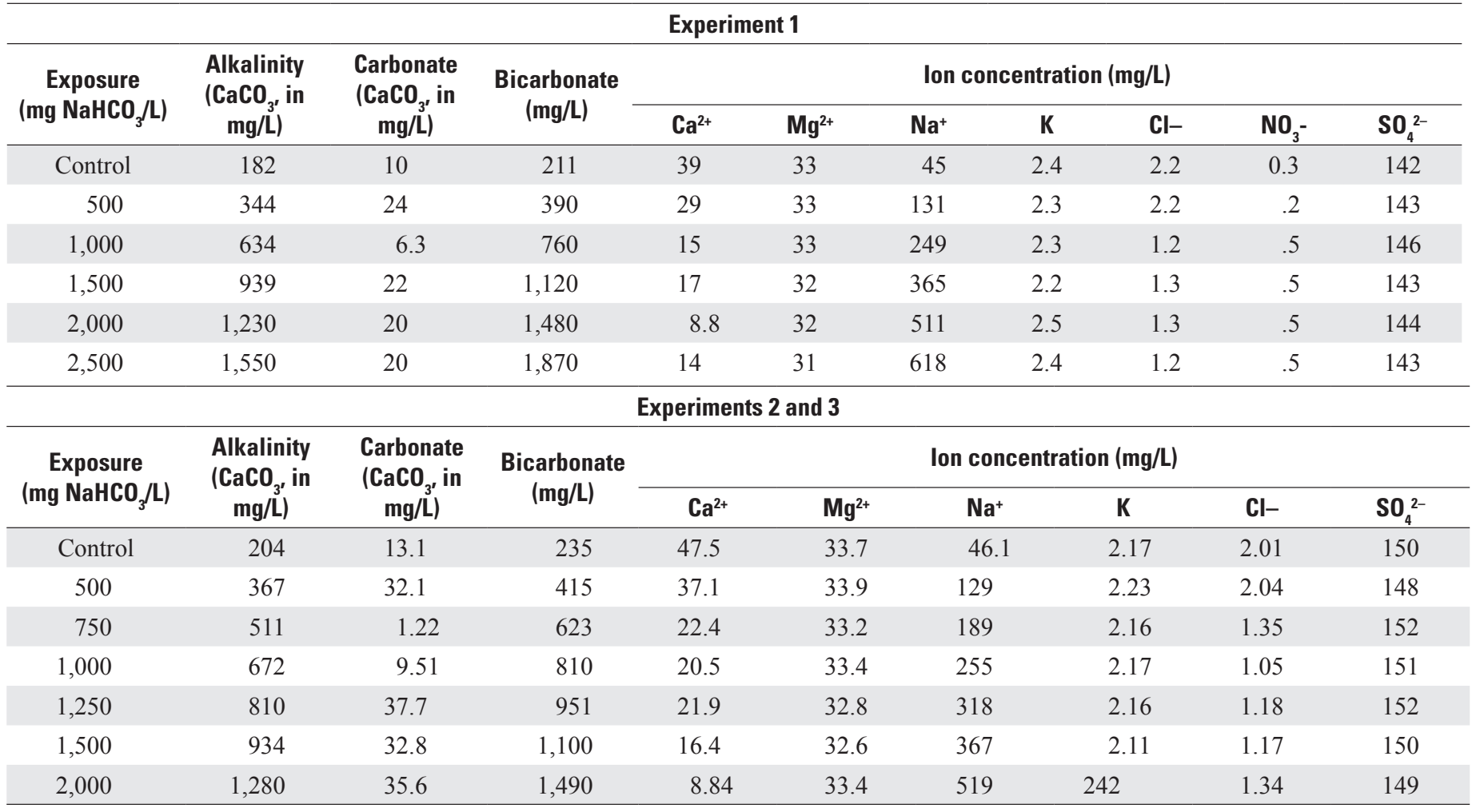

Table 2-19. Number alive and percent survival of newly transformed fatmucket mussels (Lampsilis siliquoidea) in a 96-hour acute toxicity experiment completed in reconstituted Tongue River water. Exposures presented are nominal values for sodium bicarbonate.

$\left[\mathrm{NaHCO}_{3}\right.$, sodium bicarbonate; mg/L, milligrams per liter; ( ), percent survival; --, not applicable]

\begin{tabular}{|c|c|c|c|c|c|}
\hline \multicolumn{2}{|c|}{ Experiment 1} & \multicolumn{2}{|c|}{ Experiment 2} & \multicolumn{2}{|c|}{ Experiment 3} \\
\hline $\begin{array}{c}\text { Exposure } \\
(\mathrm{mg} \mathrm{NaHCO} / \mathrm{L})\end{array}$ & $\begin{array}{l}\text { 96-hour } \\
\text { survival }\end{array}$ & $\begin{array}{c}\text { Exposure } \\
\text { (mg NaHCO} / \mathrm{L})\end{array}$ & $\begin{array}{l}\text { 96-hour } \\
\text { survival }\end{array}$ & $\begin{array}{c}\text { Exposure } \\
\text { (mg NaHCO} / \mathrm{L})\end{array}$ & $\begin{array}{l}\text { 96-hour } \\
\text { survival }\end{array}$ \\
\hline Control & $20(100)$ & Control & $20(100)$ & Control & $20(100)$ \\
\hline 500 & $19(95)$ & 500 & $20(100)$ & 500 & $20(100)$ \\
\hline 1,000 & $11(55)$ & 750 & $19(95)$ & 750 & $19(95)$ \\
\hline 1,500 & $0(0)$ & 1,000 & $16(80)$ & 1,000 & $14(70)$ \\
\hline 2,000 & $0(0)$ & 1,250 & $9(45)$ & 1,250 & $4(25)$ \\
\hline 2,500 & $0(0)$ & 1,500 & $2(10)$ & 1,500 & $0(0)$ \\
\hline-- & -- & 2,000 & $0(0)$ & 2,000 & $0(0)$ \\
\hline
\end{tabular}




\section{Rainbow Trout}

The calculated LC50 for 2-dph rainbow trout exposed to reconstituted Tongue River water was $8,070 \mathrm{mg} \mathrm{NaHCO} / \mathrm{L}$, with 95-percent CI of 7,493-8,647 $\mathrm{mg} \mathrm{NaHCO}_{3} / \mathrm{L}$. Water chemistry and survival data are presented in tables $2-20$ and $2-21$.

Table 2-20. Water chemistry measured during a 96-hour acute toxicity experiment with rainbow trout (Oncorhynchus mykiss) completed in reconstituted Tongue River water. Exposures presented are nominal concentrations of milligrams sodium bicarbonate. Sample size equals 1 for each.

$\left[\mathrm{NaHCO}_{3}\right.$, sodium bicarbonate; $\mathrm{mg} / \mathrm{L}$, milligrams per liter; $\mathrm{CaCO}_{3}$, calcium carbonate; $\mathrm{Ca}^{2+}$, calcium; $\mathrm{Mg}^{2+}$, magnesium; $\mathrm{Na}+$, sodium; $\mathrm{K}$, potassium; $\mathrm{Cl}$, chloride; $\mathrm{SO}_{4}^{2-}$, sulfate]

\begin{tabular}{|c|c|c|c|c|c|c|c|c|c|}
\hline $\begin{array}{c}\text { Exposure } \\
\text { (mg NaHCO} \\
\text { (mall) }\end{array}$ & $\begin{array}{c}\text { Alkalinity } \\
\left(\mathrm{CaCO}_{3^{\prime}} \text { in }\right. \\
\mathrm{mg} / \mathrm{L})\end{array}$ & $\begin{array}{c}\text { Carbonate } \\
\text { (CaCO, in } \\
\text { mg/L) }\end{array}$ & $\begin{array}{c}\text { Bicarbonate } \\
(\mathrm{mg} / \mathrm{L})\end{array}$ & \multicolumn{6}{|c|}{ Ion concentration (mg/L) } \\
\hline Control & 208 & 5.06 & 248 & 3.18 & 24.9 & 139 & 6.27 & 5.21 & 216 \\
\hline 4,000 & 4,020 & 512 & 4,280 & 2.38 & 15.8 & 1,680 & 5.2 & 9.62 & 141 \\
\hline 6,000 & 5,930 & 745 & 6,320 & 3.31 & 22.4 & 2,420 & 8.31 & 12.1 & 206 \\
\hline 8,000 & 6,000 & 570 & 6,560 & 2.79 & 15.8 & 2,580 & 5.66 & 12.2 & 147 \\
\hline 12,000 & 8,770 & 375 & 10,200 & 3.62 & 22.1 & 3,620 & 8.08 & 18.3 & 197 \\
\hline
\end{tabular}

Table 2-21. Number alive and percent survival of juvenile rainbow trout (Oncorhynchus mykiss) completed in reconstituted Tongue River water. Exposures presented are nominal values for sodium bicarbonate. Sample size equals 1 for each.

$\left[\mathrm{NaHCO}_{3}\right.$, sodium bicarbonate; $\mathrm{mg} / \mathrm{L}$, milligrams per liter; ( ), percent survival]

\begin{tabular}{|c|c|c|c|c|c|}
\hline $\begin{array}{c}\text { Exposure } \\
\text { (mg NaHCO} / \mathrm{L})\end{array}$ & 0 hour & 24 hours & 48 hours & 72 hours & 96 hours \\
\hline Control & $30(100)$ & $30(100)$ & $30(100)$ & $30(100)$ & $29(97)$ \\
\hline 4,000 & $30(100)$ & $30(100)$ & $29(97)$ & $29(97)$ & $29(97)$ \\
\hline 6,000 & $30(100)$ & $21(100)$ & $9(30)$ & $8(27)$ & $7(23)$ \\
\hline 8,000 & $30(100)$ & $15(50)$ & $10(33)$ & $3(10)$ & $1(3)$ \\
\hline 10,000 & $30(100)$ & $3(10)$ & $0(0)$ & $0(0)$ & $0(0)$ \\
\hline 12,000 & $30(100)$ & $0(0)$ & $0(0)$ & $0(0)$ & $0(0)$ \\
\hline
\end{tabular}




\section{Ceriodaphnia}

The calculated LC50s for C. dubia were; $1,288 \mathrm{mg}$ $\mathrm{NaHCO}_{3} / \mathrm{L}$, with a 95-percent CI of 1,171-1,406 mg $\mathrm{NaHCO}_{3} / \mathrm{L}$ in moderately hard reconstituted water; $989 \mathrm{mg}$
$\mathrm{NaHCO}_{3} / \mathrm{L}$ with 95 -percent $\mathrm{CI}$ of $910-1,069-\mathrm{mg} \mathrm{NaHCO}_{3} / \mathrm{L}$ in reconstituted Tongue River water; and $1,355 \mathrm{mg} \mathrm{NaHCO} / / \mathrm{L}$ with 95-percent $\mathrm{CI}$ of $1,257-1,453 \mathrm{mg} \mathrm{NaHCO} / \mathrm{L}$ in reconstituted Powder River water. Water chemistry and survival data are presented in tables 2-22 and 2-23.

Table 2-22. Water chemistry measured during a 48-hour acute toxicity experiment with adult (Ceriodaphnia dubia) completed in moderately hard reconstituted water, reconstituted Tongue River water, and reconstituted Powder River water. Exposures presented are nominal values for sodium bicarbonate.

$\left[\mathrm{NaHCO}_{3}\right.$, sodium bicarbonate; $\mathrm{mg} / \mathrm{L}$, milligrams per liter; $\mathrm{CaCO}_{3}$, calcium carbonate; $\mathrm{Ca}^{2+}$, calcium; $\mathrm{Mg}^{2+}$, magnesium; $\mathrm{Na}+$, sodium; $\mathrm{K}$, potassium; $\mathrm{Cl}$, chloride; $\mathrm{SO}_{4}^{2-}$, sulfate; ( ), standard error in parentheses; Mod hard, moderately hard water]

\begin{tabular}{|c|c|c|c|c|c|c|c|c|c|}
\hline \multirow{2}{*}{$\begin{array}{c}\text { Exposure } \\
(\mathrm{mg} \mathrm{NaHCO} / \mathrm{L})\end{array}$} & \multirow{2}{*}{$\begin{array}{c}\text { Alkalinity } \\
\left(\mathrm{CaCO}_{3^{\prime}} \text { in }\right. \\
\mathrm{mg} / \mathrm{L})\end{array}$} & \multirow{2}{*}{$\begin{array}{c}\text { Carbonate } \\
\left(\mathrm{CaCO}_{3^{\prime}} \text { in }\right. \\
\mathrm{mg} / \mathrm{L})\end{array}$} & \multirow{2}{*}{$\begin{array}{c}\text { Bicarbonate } \\
\text { (mg/L) }\end{array}$} & \multicolumn{6}{|c|}{ Ion concentration (mg/L) } \\
\hline & & & & $\mathrm{Ca}^{2+}$ & $\mathbf{M g}^{2+}$ & $\mathrm{Na}^{+}$ & $\mathbf{K}$ & Cl- & $\mathrm{SO}_{4}^{2-}$ \\
\hline $\begin{array}{l}\text { Mod hard } \\
\text { control }\end{array}$ & 76 & 0 & 93 & 15 & 9.0 & 21 & 7.9 & 1.4 & 96 \\
\hline 900 & 546 & 71 & 579 & 14 & 13 & 216 & 2.7 & 1.8 & 99 \\
\hline 1,300 & 736 & 140 & 727 & 8.0 & 13 & 339 & 2.4 & 1.0 & 93 \\
\hline 1,600 & 918 & 144 & 944 & 5.0 & 12 & 418 & 2.2 & 1.0 & 98 \\
\hline 2,000 & 1,136 & 288 & 1,034 & 4.0 & 12 & 476 & 2.2 & 1.2 & 96 \\
\hline 3,000 & 2,040 & 640 & 1,707 & 5.4 & 13 & 768 & 6.9 & 3.0 & 113 \\
\hline $\begin{array}{l}\text { Tongue River } \\
\text { control }\end{array}$ & 204 & 1 & 249 & 47 & 34 & 43 & 2.4 & 1.7 & 157 \\
\hline 900 & 604 & 104 & 610 & 44 & 34 & 185 & 2.0 & 2.3 & 164 \\
\hline 1,300 & 746 & 180 & 715 & 38 & 33 & 303 & 2.7 & 2.0 & 157 \\
\hline 1,600 & 900 & 236 & 810 & 30 & 33 & 390 & 2.7 & 2.2 & 162 \\
\hline 2,000 & 1,100 & 280 & 1,000 & 4.6 & 33 & 476 & 3.3 & 2.8 & 158 \\
\hline 3,000 & 1,880 & 680 & 1,460 & 5 & 32 & 765 & 4.1 & 3.9 & 171 \\
\hline $\begin{array}{l}\text { Powder River } \\
\text { control }\end{array}$ & 212 & 16 & 239 & 13 & 180 & 283 & 13 & 11 & 1,320 \\
\hline 900 & 534 & 120 & 505 & 11 & 174 & 438 & 15 & 11 & 1,430 \\
\hline 1,300 & 710 & 168 & 661 & 12 & 177 & 488 & 15 & 11 & 1,410 \\
\hline 1,600 & 912 & 276 & 776 & 12 & 176 & 583 & 16 & 11 & 1,410 \\
\hline 2,000 & 1,100 & 324 & 944 & 6.0 & 176 & 740 & 16 & 10 & 1,330 \\
\hline 3,000 & 1,860 & 720 & 1,370 & 6.0 & 176 & 931 & 17 & 12 & 1,530 \\
\hline
\end{tabular}

Table 2-23. Number of live organisms at 0, 24, and 48 hours and percent survival at 48 hours for adult Ceriodaphnia dubia completed in moderately hard reconstituted water, reconstituted Tongue River water, and reconstituted Powder River water. Exposures presented are nominal concentrations of sodium bicarbonate.

$\left[\mathrm{NaHCO}_{3}\right.$, sodium bicarbonate; $\mathrm{mg} / \mathrm{L}$, milligrams per liter]

\begin{tabular}{|c|c|c|c|c|c|c|c|c|c|c|c|c|}
\hline \multirow{3}{*}{$\begin{array}{c}\text { Exposure } \\
(\mathrm{mg} \mathrm{NaHCO} / \mathrm{L})\end{array}$} & \multicolumn{4}{|c|}{ Moderately hard reconstituted } & \multicolumn{4}{|c|}{ Reconstituted Tongue River } & \multicolumn{4}{|c|}{ Reconstituted Powder River } \\
\hline & \multicolumn{4}{|c|}{ Survival } & \multicolumn{4}{|c|}{ Survival } & \multicolumn{4}{|c|}{ Survival } \\
\hline & O hour & 24 hour & 48 hour & Percent & O hour & 24 hour & 48 hour & Percent & O hour & 24 hour & 48 hour & Percent \\
\hline 900 & 10 & 10 & 10 & 100 & 10 & 9 & 9 & 90 & 10 & 10 & 10 & 100 \\
\hline 1,300 & 10 & 9 & 9 & 90 & 10 & 9 & 5 & 50 & 10 & 7 & 7 & 70 \\
\hline 3,000 & 10 & 0 & 0 & 0 & 10 & 0 & 0 & 0 & 10 & 0 & 0 & 0 \\
\hline
\end{tabular}




\section{Chironomids}

The calculated LC50 for 1-2 week post hatch chironomid larvae exposed to reconstituted Tongue River water was $4,947 \mathrm{mg} \mathrm{NaHCO} / \mathrm{L}$, with a 95 -percent $\mathrm{CI}$ of 4,674-5,221 $\mathrm{mg} \mathrm{NaHCO} / \mathrm{L}$, and $8,014 \mathrm{mg} \mathrm{NaHCO} / \mathrm{L}$ in reconstituted Powder River water, with a 95-percent CI of 7,513-8,514 mg $\mathrm{NaHCO}_{3} / \mathrm{L}$. Water chemistry and survival data are presented in tables 2-24 and 2-25.

Table 2-24. Water chemistry measured in concentrated stock solutions and reconstituted (control) Tongue River and Powder River waters used to make exposure concentrations for a 48-hour acute toxicity experiments with chironomid larvae (Chironomus dilutus). Sample size equals one for each. Exposures presented are nominal concentrations of sodium bicarbonate.

$\left[\mathrm{NaHCO}_{3}\right.$, sodium bicarbonate; $\mathrm{mg} / \mathrm{L}$, milligrams per liter; $\mathrm{CaCO}_{3}$, calcium carbonate; $\mathrm{Ca}^{2+}$, calcium; $\mathrm{Mg}^{2+}$, magnesium; $\mathrm{Na}+$, sodium; $\mathrm{K}$, potassium,; $\mathrm{Cl}-$, chloride; $\mathrm{SO}_{4}^{2-}$, sulfate; <. less than 1]

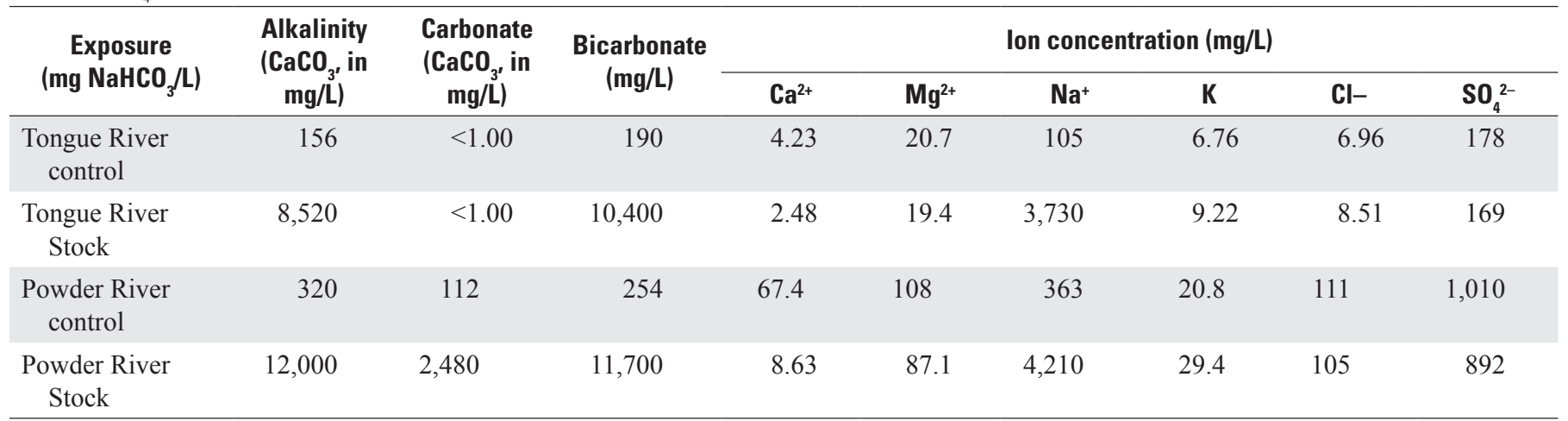

Table 2-25. Number alive and percent survival of chironomid larvae (Chironomus dilutus) in a 48-hour acute toxicity experiment completed in reconstituted (control) Tongue and Powder River waters. Exposures presented are nominal concentrations of sodium bicarbonate.

[ $\mathrm{NaHCO}_{3}$, sodium bicarbonate; $\mathrm{mg} / \mathrm{L}$, milligrams per liter; ( ), percent survival]

\begin{tabular}{|c|c|c|c|}
\hline $\begin{array}{c}\text { Exposure } \\
\text { (mg NaHCO } / \text { L) }\end{array}$ & 0 hour & 24 hours & 48 hours \\
\hline Tongue River control & $40(100)$ & $39(98)$ & $39(98)$ \\
\hline 4,000 & $40(100)$ & $38(95)$ & $38(95)$ \\
\hline 6,000 & $40(100)$ & $38(95)$ & $33(83)$ \\
\hline 8,000 & $40(100)$ & $13(33)$ & $0(0)$ \\
\hline 12,000 & $40(100)$ & $0(0)$ & $0(0)$ \\
\hline 16,000 & $40(100)$ & $0(0)$ & $0(0)$ \\
\hline Powder River control & $40(100)$ & $40(100)$ & $38(95)$ \\
\hline 4,000 & $40(100)$ & $39(98)$ & $39(98)$ \\
\hline 6,000 & $40(100)$ & $38(95)$ & $36(90)$ \\
\hline 8,000 & $40(100)$ & $33(83)$ & $18(45)$ \\
\hline 12,000 & $40(100)$ & $0(100)$ & $0(97)$ \\
\hline 16,000 & $40(100)$ & $0(100)$ & $0(97)$ \\
\hline
\end{tabular}




\section{Tubifex}

The calculated LC50 for Tubifex exposed to reconstituted Tongue River water was 3,297 $\mathrm{mg} \mathrm{NaHCO}_{3} / \mathrm{L}$, with upper and lower 95-percent $\mathrm{CI}$ of 3,121-3,473 $\mathrm{mg} \mathrm{NaHCO}_{3} / \mathrm{L}$, and
3,367 mg/L $\mathrm{NaHCO}_{3} / \mathrm{L}$, with upper and lower 95-percent CI of 3,188 and 3,547 $\mathrm{mg} \mathrm{NaHCO}_{3} / \mathrm{L}$ with reconstituted Powder River water. Water chemistry and survival data are presented in tables 2-26 and 2-27.

Table 2-26. Water chemistry measured in concentrated stock solutions of 5,000 milligrams sodium bicarbonate per liter and reconstituted (control) Tongue and Powder River waters used to make exposure concentrations for 96-hour acute toxicity experiments with Tubifex worms (Tubifex tubifex). Exposures presented are nominal values for sodium bicarbonate.

$\left[\mathrm{NaHCO}_{3}\right.$, sodium bicarbonate; $\mathrm{mg} / \mathrm{L}$, milligrams per liter; $\mathrm{CaCO}_{3}$, calcium carbonate; $\mathrm{Ca}^{2+}$, calcium; $\mathrm{Mg}^{2+}$, magnesium; $\mathrm{Na}{ }^{+}$, sodium; $\mathrm{K}$, potassium; $\mathrm{Cl}-$, chloride; $\mathrm{NO}_{3}^{-}$, nitrate; $\mathrm{SO}_{4}^{2-}$, sulfate]

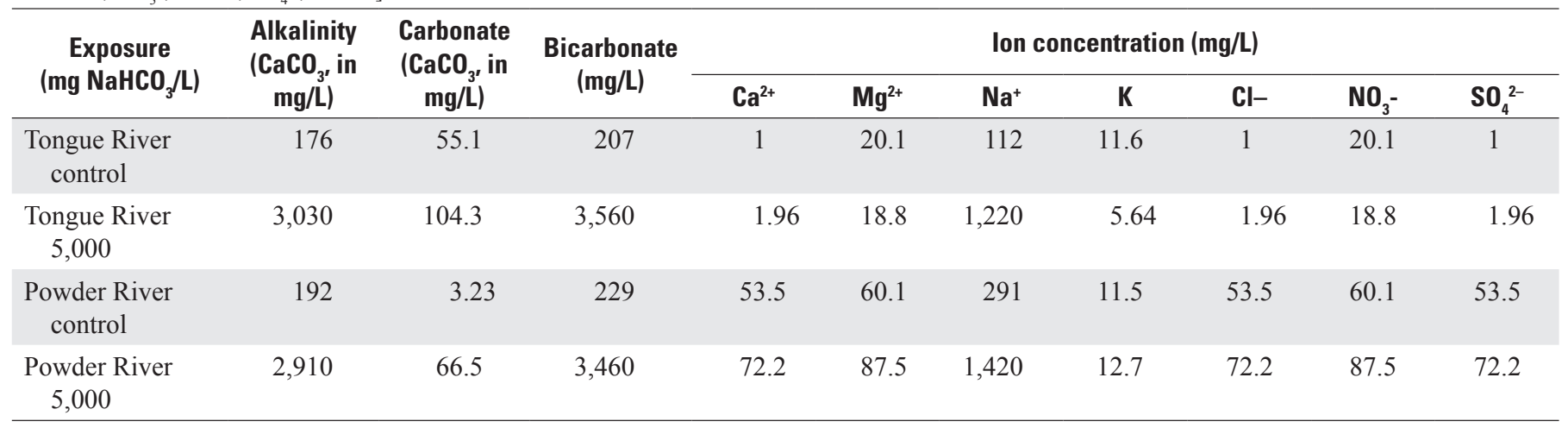

Table 2-27. Number alive and percent survival of Tubifex worms (Tubifex tubifex) in 96-hour acute toxicity experiments completed in reconstituted (control) Tongue and Powder River waters. Exposures presented are nominal values for sodium bicarbonate.

$\left[\mathrm{NaHCO}_{3}\right.$, sodium bicarbonate; $\mathrm{mg} / \mathrm{L}$, milligrams per liter; ( ), percent survival]

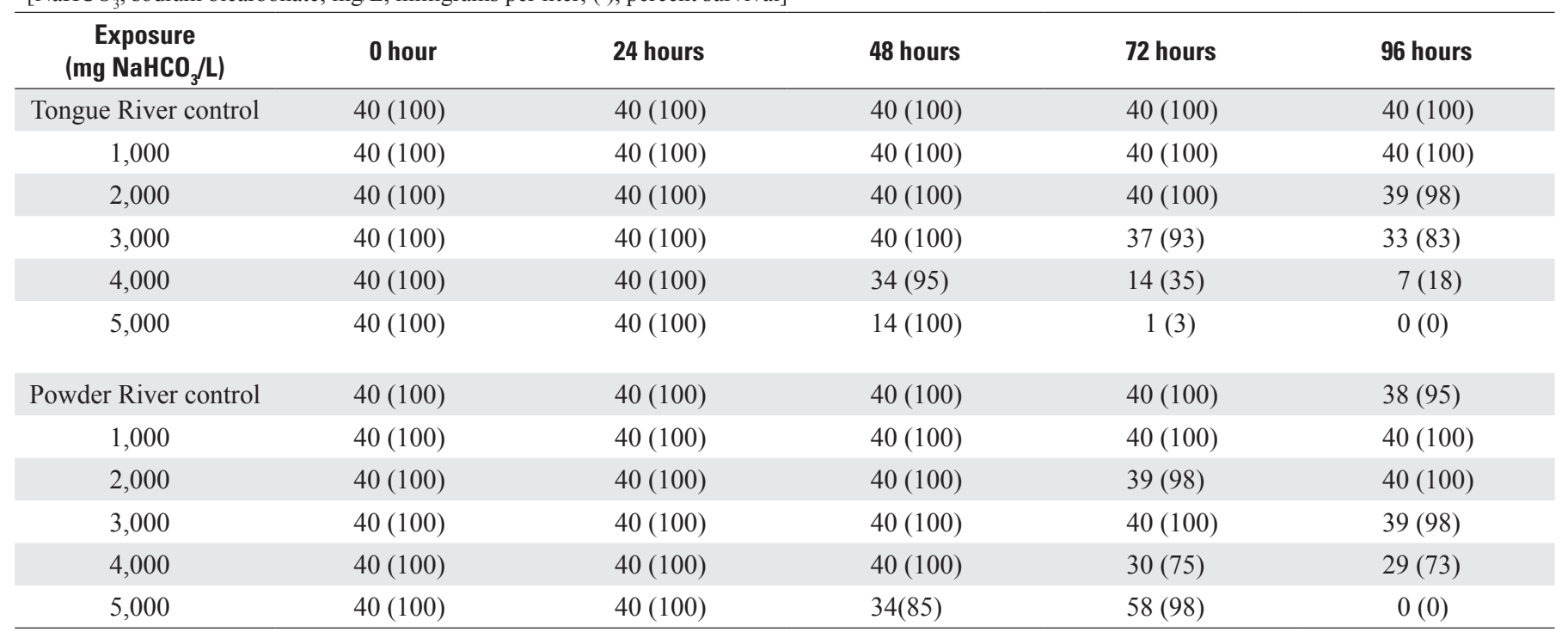




\section{Amphipods}

The calculated LC50 for Hyalella azteca exposed to reconstituted Tongue River water was $1,419 \mathrm{mg} / \mathrm{L} \mathrm{NaHCO}_{3} / \mathrm{L}$, with 95-percent CI of 1,340-1,4982 $\mathrm{mg} \mathrm{NaHCO}_{3} / \mathrm{L}$. It should be noted that this LC50 may be affected by low chloride $\left(\mathrm{Cl}^{-}\right)$ concentrations in the reconstituted Tongue River water. An LC50 was calculated in Powder River reconstituted water as $3,851 \mathrm{mg} \mathrm{NaHCO} / \mathrm{L}$. However, the low mortality in the largest concentration $(2,000 \mathrm{mg} / \mathrm{L})$ and the resulting large $\mathrm{CI}$ of $-3,642-9,450 \mathrm{mg} / \mathrm{L}$, make this number suspect. Water chemistry and survival data are presented in tables 2-28 and 2-29.

Table 2-28. Water chemistry measured in concentrated stock solutions of sodium bicarbonate and reconstituted (control) Tongue and Powder River waters used to make exposure concentrations (also presented) for 96-hour acute toxicity experiments with adult amphipods (Hyalella azteca). Exposures presented are nominal values for sodium bicarbonate. Sample size equals one for each.

$\left[\mathrm{NaHCO}_{3}\right.$, sodium bicarbonate; $\mathrm{mg} / \mathrm{L}$, milligrams per liter; $\mathrm{CaCO}_{3}$, calcium carbonate; $\mathrm{Ca}^{2+}$, calcium, $\mathrm{Mg}^{2+} ;$ magnesium; $\mathrm{Na}{ }^{+}$, sodium; $\mathrm{K}$, potassium; $\mathrm{Cl}-$, chloride; $\mathrm{SO}_{4}^{2-}$, sulfate]

\begin{tabular}{|c|c|c|c|c|c|c|c|c|c|}
\hline \multirow{2}{*}{$\begin{array}{c}\text { Exposure } \\
(\mathrm{mg} \mathrm{NaHCO} / \mathrm{L})\end{array}$} & \multirow{2}{*}{$\begin{array}{c}\text { Alkalinity } \\
\left(\mathrm{CaCO}_{3^{\prime}} \text { in }\right. \\
\mathrm{mg} / \mathrm{L})\end{array}$} & \multirow{2}{*}{$\begin{array}{l}\text { Carbonate } \\
\left(\mathrm{CaCO}_{3^{\prime}} \text { in }\right. \\
\mathrm{mg} / \mathrm{L})\end{array}$} & \multirow{2}{*}{$\begin{array}{c}\text { Bicarbonate } \\
\text { (mg/L) }\end{array}$} & \multicolumn{6}{|c|}{ Ion concentration (mg/L) } \\
\hline & & & & $\mathrm{Ca}^{2+}$ & $\mathbf{M g}^{2+}$ & $\mathrm{Na}^{+}$ & K & Cl- & $\mathrm{SO}_{4}{ }^{2}-$ \\
\hline Tongue River control & 156 & 8.98 & 147 & 2 & 19.6 & 108 & 6.03 & 3.32 & 177 \\
\hline Tongue River stock & 1,210 & 90 & 1,120 & 2.53 & 18.9 & 553 & 7.0 & 3.7 & 174 \\
\hline Powder River control & 199 & 6.15 & 192 & 55.4 & 90.1 & 288 & 13 & 97.5 & 829 \\
\hline \multirow[t]{8}{*}{ Powder River stock } & 1,110 & 33.7 & 1,070 & 53.9 & 88.9 & 724 & 13.8 & 103 & 878 \\
\hline & \multicolumn{2}{|c|}{$\begin{array}{l}\text { Tongue River } \\
\text { nominal } \\
\text { (mg NaHCO} / \mathrm{L} \text { ) }\end{array}$} & $\begin{array}{c}\text { Measured } \\
\text { mg NaHCO }_{3}(\mathrm{~L})\end{array}$ & \multicolumn{2}{|c|}{$\begin{array}{c}\text { Tongue River } \\
\text { nominal } \\
\text { (mg NaHCO} / \mathrm{L} \text { ) }\end{array}$} & \multicolumn{2}{|c|}{$\begin{array}{c}\text { Measured } \\
\text { (mg NaHCO} / \text { L) }\end{array}$} & & \\
\hline & \multicolumn{2}{|l|}{ Control } & 264 & \multicolumn{2}{|c|}{ Control } & \multicolumn{2}{|c|}{486} & & \\
\hline & \multicolumn{2}{|l|}{1,000} & 879 & \multicolumn{2}{|c|}{1,000} & \multicolumn{2}{|c|}{923} & & \\
\hline & \multicolumn{2}{|l|}{1,250} & 1,100 & \multicolumn{2}{|c|}{1,250} & \multicolumn{2}{|c|}{1,150} & & \\
\hline & \multicolumn{2}{|l|}{1,500} & 1,320 & \multicolumn{2}{|c|}{1,500} & \multicolumn{2}{|c|}{1,380} & & \\
\hline & \multicolumn{2}{|l|}{1,750} & 1,540 & \multicolumn{2}{|c|}{1,750} & \multicolumn{2}{|c|}{1,620} & & \\
\hline & \multicolumn{2}{|l|}{2,000} & 1,760 & \multicolumn{2}{|c|}{2,000} & \multicolumn{2}{|c|}{1,850} & & \\
\hline
\end{tabular}

Table 2-29. Number alive and percent survival of adult amphipods (Hyalella azteca) in 96-hour acute toxicity experiments completed in reconstituted (control) Tongue and Powder River waters. Exposures presented are nominal values for sodium bicarbonate.

$\left[\mathrm{NaHCO}_{3}\right.$, sodium bicarbonate; $\mathrm{mg} / \mathrm{L}$, milligrams per liter; ( ), percent survival $]$

\begin{tabular}{|c|c|c|c|c|c|}
\hline $\begin{array}{c}\text { Exposure } \\
\left(\mathrm{mg} \mathrm{NaHCO}_{3} / \mathrm{L}\right)\end{array}$ & O hour & 24 hours & 48 hours & 72 hours & 96 hours \\
\hline Tongue River control & $40(100)$ & $40(100)$ & $40(100)$ & $40(100)$ & $40(100)$ \\
\hline 1,250 & $40(100)$ & $40(100)$ & $40(100)$ & $34(85)$ & $29(73)$ \\
\hline 1,500 & $40(100)$ & $40(100)$ & $40(100)$ & $31(78)$ & $22(55)$ \\
\hline Powder River control & $40(100)$ & $40(100)$ & $40(100)$ & $40(100)$ & $40(100)$ \\
\hline 1,000 & $40(100)$ & $40(100)$ & $40(100)$ & $40(100)$ & $40(100)$ \\
\hline 1,250 & $40(100)$ & $40(100)$ & $39(98)$ & $39(98)$ & $38(95)$ \\
\hline
\end{tabular}




\section{Shovelnose Sturgeon}

The calculated LC50 for yolk-sac fry shovelnose sturgeon exposed to Yellowstone River water was $1,038 \mathrm{mg} \mathrm{NaHCO}_{3} / \mathrm{L}$.
A reliable 95-percent CI could not be calculated. Water chemistry and survival data are presented in tables $2-30$ and $2-31$.

Table 2-30. Water chemistry measured during a 96-hour acute toxicity experiment with shovelnose sturgeon (Scaphirhynchus platorynchus) completed in Yellowstone River water (control). Exposures presented are nominal values for sodium bicarbonate. Sample size equals one for each.

$\left[\mathrm{NaHCO}_{3}\right.$, sodium bicarbonate; $\mathrm{mg} / \mathrm{L}$, milligrams per liter; $\mathrm{CaCO}_{3}$, calcium carbonate; $\mathrm{Ca}^{2+}$, calcium; $\mathrm{Mg}^{2+}$, magnesium; $\mathrm{Na}$, sodium; $\mathrm{K}$, potassium; $\mathrm{Cl}-$, chloride; $\mathrm{SO}_{4}^{2-}$, sulfate; $<$, less than 1]

\begin{tabular}{|c|c|c|c|c|c|c|c|c|c|}
\hline \multirow{2}{*}{$\begin{array}{c}\text { Exposure } \\
(\mathrm{mg} \mathrm{NaHCO} / \mathrm{L})\end{array}$} & \multirow{2}{*}{$\begin{array}{c}\text { Alkalinity } \\
\left(\mathrm{CaCO}_{3^{\prime}} \text { in }\right. \\
\mathrm{mg} / \mathrm{L})\end{array}$} & \multirow{2}{*}{$\begin{array}{c}\text { Carbonate } \\
\left(\mathrm{CaCO}_{3^{\prime}} \text { in }\right. \\
\mathrm{mg} / \mathrm{L})\end{array}$} & \multirow{2}{*}{$\begin{array}{l}\text { Bicarbonate } \\
\text { (mg/L) }\end{array}$} & \multicolumn{6}{|c|}{ Ion concentration (mg/L) } \\
\hline & & & & $\mathrm{Ca}^{2+}$ & $\mathbf{M g}^{2+}$ & $\mathrm{Na}^{+}$ & K & $\mathrm{Cl}-$ & $\mathrm{SO}_{4}{ }^{2}-$ \\
\hline Control & 94.5 & $<1$ & 115 & 3.75 & 10.75 & 65.95 & 3.8 & 3.2 & 109.5 \\
\hline 625 & 469 & 6 & 565 & 3.6 & 10.7 & 225 & 4.2 & 3 & 109.5 \\
\hline 1,250 & 843 & 11 & 1,020 & 3.4 & 10.6 & 384 & 4.5 & 2.9 & 109.6 \\
\hline 2,500 & 1,591 & 20 & 1,920 & 3 & 10.5 & 710 & 5.3 & 2.5 & 109.6 \\
\hline 5,000 & 3,087 & 47 & 3,720 & 1.9 & 10.4 & 1,338 & 6.7 & 1.9 & 109.75 \\
\hline 10,000 & 6,080 & 80 & 7,320 & .5 & 10.1 & 2,610 & 9.7 & .5 & 110 \\
\hline
\end{tabular}

Table 2-31. Number alive and percent survival of 1-day-post-hatch shovelnose sturgeon (Scaphirhynchus platorynchus) completed in Yellowstone River water (control). Exposures presented are nominal values for sodium bicarbonate.

$\left[\mathrm{NaHCO}_{3}\right.$, sodium bicarbonate; $\mathrm{mg} / \mathrm{L}$, milligrams per liter; ( ), percent survival $]$

\begin{tabular}{|c|c|c|c|c|c|}
\hline $\begin{array}{c}\text { Exposure } \\
\left(\mathrm{mg} \mathrm{NaHCO}_{3} / \mathrm{L}\right)\end{array}$ & 0 hour & 24 hours & 48 hours & 72 hours & 96 hours \\
\hline Control & $20(100)$ & $20(100)$ & $20(100)$ & $20(100)$ & $20(100)$ \\
\hline 1,250 & $20(100)$ & $20(100)$ & $20(100)$ & $12(60)$ & $11(55)$ \\
\hline 2,500 & $20(100)$ & $20(100)$ & $20(100)$ & $0(0)$ & $0(0)$ \\
\hline
\end{tabular}




\section{Walleye}

The calculated LC50 for 1-dph walleye exposed to Yellowstone River water was 3,249 $\mathrm{mg} \mathrm{NaHCO}_{3} / \mathrm{L}$, with 95-percent CI of 2,850-3,648 $\mathrm{mg} \mathrm{NaHCO} / \mathrm{L}$. Water chemistry and survival data are presented in tables 2-32 and 2-33.

Table 2-32. Water chemistry measured during a 96-hour acute toxicity experiment with 1-day-post-hatch walleye (Sander vitreus) completed in Yellowstone River water (control). Exposures presented are nominal values for sodium bicarbonate.

$\left[\mathrm{NaHCO}_{3}\right.$, sodium bicarbonate; $\mathrm{mg} / \mathrm{L}$; milligrams per liter, $\mathrm{CaCO}_{3}$, calcium carbonate; $\mathrm{Ca}^{2+}$, calcium; $\mathrm{Mg}^{2+}$, magnesium; $\mathrm{Na}$, sodium; $\mathrm{K}$, potassium; $\mathrm{Cl}-$, chloride; $\mathrm{SO}_{4}^{2-}$, sulfate]

\begin{tabular}{|c|c|c|c|c|c|c|c|c|c|c|}
\hline $\begin{array}{c}\text { Exposure } \\
\text { (mg NaHCO} / \mathrm{L})\end{array}$ & $\begin{array}{c}\text { Alkalinity } \\
\left(\mathrm{CaCO}_{3^{\prime}} \text { in }\right. \\
\mathrm{mg} / \mathrm{L})\end{array}$ & $\begin{array}{c}\text { Carbonate } \\
\left(\mathrm{CaCO}_{3^{\prime}} \text { in }\right. \\
\mathrm{mg} / \mathrm{L})\end{array}$ & $\begin{array}{c}\text { Bicarbonate } \\
\text { (mg/L) }\end{array}$ & \multicolumn{7}{|c|}{ Ion concentration $(\mathrm{mg} / \mathrm{L})$} \\
\hline Control & 172 & 1 & 209 & 44.5 & 20.5 & 142 & 6.1 & 17.3 & 217 & 44.5 \\
\hline 625 & 519 & 5.9 & 626 & 41.7 & 19.2 & 289 & 5.7 & 16.2 & 203 & 41.7 \\
\hline 1,250 & 866 & 10.9 & 1,010 & 38.9 & 17.9 & 435 & 5.3 & 15.1 & 190 & 38.9 \\
\hline 2,500 & 1,560 & 20.7 & 1,880 & 33.4 & 15.4 & 729 & 4.6 & 13.0 & 163 & 33.4 \\
\hline
\end{tabular}

Table 2-33. Number alive and percent survival of 1-day-post-hatch walleye (Sander vitreus) completed in Yellowstone River water (control). Exposures presented are nominal values for sodium bicarbonate.

$\left[\mathrm{NaHCO}_{3}\right.$, sodium bicarbonate; $\mathrm{mg} / \mathrm{L}$, milligrams per liter; ( ), percent survival]

\begin{tabular}{|c|c|c|c|c|c|}
\hline $\begin{array}{c}\text { Exposure } \\
\text { (mg NaHCO} / \mathrm{L})\end{array}$ & 0 hour & 24 hours & 48 hours & 72 hours & 96 hours \\
\hline Control & $20(100)$ & $20(100)$ & $20(100)$ & $20(100)$ & $20(100)$ \\
\hline 1,250 & $20(100)$ & $20(100)$ & $20(100)$ & $20(100)$ & $19(95)$ \\
\hline 2,500 & $20(100)$ & $20(100)$ & $20(100)$ & $20(100)$ & $19(95)$ \\
\hline
\end{tabular}




\section{Northern Pike}

The calculated LC50 for 1-dph northern pike exposed to Yellowstone River water was $>8,000 \mathrm{mg} \mathrm{NaHCO}_{3} / \mathrm{L}$, no confidence interval could be calculated. Water chemistry and survival data are presented in tables 2-34 and 2-35.

Table 2-34. Water chemistry measured during a 96-hour acute toxicity experiment with 2-day-post-hatch northern pike (Esox lucius) completed in Yellowstone River water (control). Exposures presented are nominal values for sodium bicarbonate.

$\left[\mathrm{NaHCO}_{3}\right.$, sodium bicarbonate; $\mathrm{mg} / \mathrm{L}$, milligrams per liter; $\mathrm{CaCO}_{3}$, calcium carbonate; $\mathrm{Ca}^{2+}$, calcium; $\mathrm{Mg}^{2+}$, magnesium; $\mathrm{Na}{ }^{+}$, sodium; $\mathrm{Cl}-$, chloride; $\mathrm{SO}_{4}^{2-}$, sulfate; ( ), range; --, one sample was measured, no range provided. Concentrations are means unless no range is provided]

\begin{tabular}{|c|c|c|c|c|c|c|c|c|}
\hline \multirow{2}{*}{$\begin{array}{c}\text { Exposure } \\
(\mathrm{mg} \mathrm{NaHCO} / \mathrm{L})\end{array}$} & \multirow{2}{*}{$\begin{array}{c}\text { Alkalinity } \\
\left(\mathrm{CaCO}_{3^{\prime}} \text { in }\right. \\
\mathrm{mg} / \mathrm{L})\end{array}$} & \multirow{2}{*}{$\begin{array}{c}\text { Carbonate } \\
\text { (CaCO, in } \\
\text { mg/L) }\end{array}$} & \multirow{2}{*}{$\begin{array}{l}\text { Bicarbonate } \\
\text { (mg/L) }\end{array}$} & \multicolumn{5}{|c|}{ Ion concentration (mg/L) } \\
\hline & & & & $\mathrm{Ca}^{2+}$ & $\mathbf{M g}^{2+}$ & $\mathrm{Na}^{+}$ & Cl- & $\mathrm{SO}_{4}^{2}-$ \\
\hline Control & $\begin{array}{c}89.3 \\
(82-102)\end{array}$ & $\begin{array}{c}0 \\
(0.0-0.0)\end{array}$ & $\begin{array}{c}108.9 \\
(100-124)\end{array}$ & 4 & 18.8 & 48.2 & 7.59 & 121 \\
\hline 500 & $\begin{array}{c}357 \\
(348-364)\end{array}$ & $\begin{array}{c}10.7 \\
(0-32)\end{array}$ & $\begin{array}{c}423 \\
(385-444)\end{array}$ & -- & -- & -- & -- & -- \\
\hline 1,000 & $\begin{array}{c}627 \\
(604-640)\end{array}$ & $\begin{array}{c}18.7 \\
(0-56)\end{array}$ & $\begin{array}{c}741 \\
(668-780)\end{array}$ & 3.87 & 18.6 & 173 & 7.8 & 121 \\
\hline 2,000 & $\begin{array}{c}1,178 \\
(1160-1210)\end{array}$ & $\begin{array}{c}56 \\
(0-128)\end{array}$ & $\begin{array}{c}1,368 \\
(1,260-1,430)\end{array}$ & -- & -- & -- & -- & -- \\
\hline 4,000 & $\begin{array}{c}2,468 \\
(2300-2560)\end{array}$ & $\begin{array}{c}211 \\
(120-240)\end{array}$ & $\begin{array}{c}2,752 \\
(2,610-2,830)\end{array}$ & 3.75 & 18.5 & 297 & 8 & 121 \\
\hline 8,000 & $\begin{array}{c}4,181 \\
(4,120-4,220)\end{array}$ & $\begin{array}{c}336 \\
(120-464)\end{array}$ & $\begin{array}{c}4,690 \\
(3,650-4,100)\end{array}$ & -- & -- & -- & -- & -- \\
\hline
\end{tabular}

Table 2-35. Number alive and percent survival of 2-day-post-hatch northern pike (Esox lucius) completed in Yellowstone River water (control). Exposures presented are nominal values for sodium bicarbonate.

[ $\mathrm{NaHCO}_{3}$, sodium bicarbonate; $\mathrm{mg} / \mathrm{L}$, milligrams per liter; ( ), percent survival]

\begin{tabular}{|c|c|c|c|c|c|}
\hline $\begin{array}{c}\text { Exposure } \\
\left(\mathrm{mg} \mathrm{NaHCO}_{3} / \mathrm{L}\right)\end{array}$ & 0 hour & 24 hours & 48 hours & 72 hours & 96 hours \\
\hline Control & $40(100)$ & $40(100)$ & $40(100)$ & $40(100)$ & $40(100)$ \\
\hline 1,000 & $40(100)$ & 39 (97) & 39 (97) & 39 (97) & $38(95)$ \\
\hline 2,000 & $40(100)$ & $40(100)$ & $40(100)$ & $40(100)$ & $40(100)$ \\
\hline
\end{tabular}




\section{Discussion}

Sodium bicarbonate generally has been considered a relatively harmless substance, and there has been very little research into its toxicity to aquatic organisms. This is likely because of the large concentrations required to induce toxicity when compared to other potential toxicants such as heavy metals, ammonia and pesticides. Because of the lack of research into the toxicity of salts, total dissolved solids (TDS) and conductivity have been used to predict toxicity; however, the toxicity of different salts can be quite variable and TDS and conductivity are not always good predictors of toxicity (Burnham and Peterka, 1975; Dickerson and others, 1996; WeberScannell and Duffy, 2007).

In a series of experiments to predict the toxicity of salts to aquatic organisms, Mount and others (1997) calculated a 48-h LC50 of 1,020 mg NaHCO$/ 2$ for $C$. dubia exposed to moderately hard reconstituted water. Experiments in this study with $C$. dubia resulted in an $\mathrm{LC} 50$ of $989 \mathrm{mg} \mathrm{NaHCO} / \mathrm{L}$ in simulated Tongue River water, and $1,355 \mathrm{mg} \mathrm{NaHCO} / \mathrm{L}$ in simulated Powder River water. Mount and others (1997) also exposed FHM to $\mathrm{NaHCO}_{3}$ and determined them to be more sensitive than C. dubia, with a $96-\mathrm{h}$ LC50 of $<850 \mathrm{mg}$ $\mathrm{NaHCO}_{3} / \mathrm{L}$. In the present study experiments, FHM were not more sensitive than $C$. dubia, and the LC50 concentrations were $1,749 \mathrm{mg} \mathrm{NaHCO}_{3} / \mathrm{L}$ in simulated Tongue River water and 2,079 $\mathrm{mg} \mathrm{NaHCO}_{3} / \mathrm{L}$ in simulated Powder River water. Overall, results of the present study demonstrate that $\mathrm{NaHCO}_{3}$ is acutely toxic to aquatic organisms with LC50 or EC50 values that range from $989->8,000 \mathrm{mg} / \mathrm{L}$. Further, the LC50 values for 6 of the 13 species utilized were less than 2,000 $\mathrm{mg}$ $\mathrm{NaHCO}_{3} / \mathrm{L}$. These results are comparable to those obtained by Mount and others (1997).

The Salinity Toxicity Relationship (STR) model, developed by Mount and Gulley (1992) and validated by Tietge and others (1997), predicts LC50 values for combinations of salts. Using the measured water chemistry from the present study of fathead minnow experiments in reconstituted Tongue River water, the STR model predicted 96-h LC50 of 1,590 $\mathrm{mg} \mathrm{NaHCO} / \mathrm{L}$ for FHM, which is comparable to our measured $96-\mathrm{h} \mathrm{LC} 50$ of $1,749 \mathrm{mg} \mathrm{NaHCO} / \mathrm{L}$. The STR model predicted 48 -h LC50 of $1,571 \mathrm{mg} \mathrm{NaHCO} / \mathrm{L}$ for C. dubia in moderately hard reconstituted water; $1,776 \mathrm{mg} \mathrm{NaHCO} / \mathrm{L}$ in reconstituted Tongue River water; and $1,575 \mathrm{mg} \mathrm{NaHCO}_{3} / \mathrm{L}$ in reconstituted Powder River water. Predicted values in the STR model were all greater than the LC50 values calculated with the TRAP model in the present study of $1,288 \mathrm{mg} \mathrm{NaHCO}_{3} / \mathrm{L}$ in moderately hard reconstituted water; $989 \mathrm{mg} \mathrm{NaHCO} / \mathrm{L}$ in reconstituted Tongue River water; and $1,355 \mathrm{mg} \mathrm{NaHCO}_{3} / \mathrm{L}$ in reconstituted Powder River water. The STR model was conservative for FHM in reconstituted Tongue River water; however, the STR model under-predicted toxicity to C. dubia in all reconstituted waters.

Dwyer and others (2005) observed that no single species was the most sensitive to a series of chemicals. Common experimental organisms such as rainbow trout are more tolerant of salinity, but are sensitive to dissolved metals or pesticides, whereas another common experimental organism, the FHM is less tolerant of salinity but more tolerant of dissolved metals. The relative tolerance to salinity of different fish species has been offered as a predictor of fish assemblages in prairie streams (Ostrand and Wilde, 2004). Although the concentrations of $\mathrm{NaHCO}_{3}$ required to induce toxicity appear larger than other commonly tested compounds, concentrations of $\mathrm{NaHCO}_{3}$ can exceed 3,000 $\mathrm{mg} / \mathrm{L}$ in untreated CBNG produced waters (Patz and others, 2004). This concentration is greater than the LC50 values reported for the majority of species exposed to $\mathrm{NaHCO}_{3}$ in the present study.

Mount and others (1997) demonstrated that the toxicity of sodium and calcium salts was caused by the co-occurring anions (specifically $\mathrm{Cl}^{-}$, sulfate, and $\mathrm{HCO}_{3}^{-}$). In the Tongue and Powder River waters that were simulated in the present experiments, $\mathrm{HCO}_{3}^{-}$was the predominant co-occurring anion. Therefore, it is likely that the primary source of toxicity of $\mathrm{NaHCO}_{3}$ can be attributed to $\mathrm{HCO}_{3}^{-}$.

Toxicity from $\mathrm{NaHCO}_{3}$ likely is caused by disruption of the mechanisms responsible for ionic regulation. Ionoregulation in aquatic organisms is controlled, in part, by chloride cells, also known as ionocytes or mitochondria rich cells. Located within the gills and the epithelium of fish, invertebrates and amphibians, ionocytes are densely packed with mitochondria and use energy to "pump" ions across the cell membrane (Hobe and others, 1984; Perry, 1997; Wilson and Laurent, 2002). An enzyme involved in this process, sodiumpotassium adenosine triphosphatase, $\left(\mathrm{Na} / \mathrm{K}^{+}\right.$ATPase), also known as the $\mathrm{Na} / \mathrm{K}^{+}$pump, likely is affected by exposure to $\mathrm{NaHCO}_{3}$ (see chapter 3 in this report). In addition to, or in conjunction with ionoregulation, chloride cells also play a role in maintaining acid/base balance (Sullivan and others, 1995; Perry, 1997; Gilmour and Perry, 2009). Chloride cells excrete $\mathrm{HCO}_{3}^{-}$in exchange for $\mathrm{Cl}^{-}$and expand in size when blood alkalosis occurs, and the rapid exchange of $\mathrm{HCO}_{3}{ }^{-}$and $\mathrm{Cl}^{-}$acts to reduce blood alkalinity (Wilson and Laurent, 2002). In fish, the acid/base balance is controlled exclusively through the exchange of $\mathrm{H}^{+}$and $\mathrm{HCO}_{3}{ }^{-}$with $\mathrm{Na}^{+}$and $\mathrm{Cl}^{-}$(Gilmour and Perry, 2009).

Bicarbonate also is involved in respiration. Carbon dioxide $\left(\mathrm{CO}_{2}\right)$ produced during respiration is converted into $\mathrm{HCO}_{3}^{-}$, which allows for the removal of $\mathrm{CO}_{2}$ and the maintenance of $\mathrm{pH}$ balance in the blood and hemolymph (Haswell and others, 1980; Burnett and McMahon, 1985; Randall and Tsui, 2006). Because $\mathrm{HCO}_{3}^{-}$may be excreted across the gill membrane for respiration and as part of the acid/base regulatory mechanism, it is possible that the inability to excrete $\mathrm{HCO}_{3}^{-}$because of elevated concentrations in the exposure water during the experiments in this study, ultimately, caused acute mortality. Hoke and others (1992) completed experiments to determine the toxicity of alkalinity to C. dubia, and concluded that $\mathrm{HCO}_{3}{ }_{3}^{-}$was toxic to aquatic organisms when concentrations were sufficiently large enough to interfere with this exchange of $\mathrm{Cl}^{-}$. 
The function of chloride cells for ionoregulation and to maintain acid/base balance appears to be interrelated (Wilson and Laurent, 2002). Exposure to brackish water resulted in an increase in the number of chloride cells, indicating a response to maintain ionic balance similar to alkalosis and acidosis (Wilson and Laurent, 2002). The response to increased salinity was an increase in the size and number of chloride cells, which are actively driving the transport of ions across the gill membrane to maintain ionic balance. The mortality associated with exposure to $\mathrm{NaHCO}_{3}$, in the present study, likely resulted from the inability of the organisms to rapidly compensate for increased alkalinity and the subsequent disruption of ionoregulation and acid/base balance, which has been observed in previous studies (Wilson and Laurent 2002; Gilmour and Perry, 2009).

The variability in the sensitivity to $\mathrm{NaHCO}_{3}$ among organisms has not been well researched; however, it has been hypothesized that differences in salinity tolerance are a result of evolutionary history of the organism (Wurts, 1998; Nielson and others, 2003). For example, salmonid fishes that evolved in environments with highly variable salinity, such as estuaries, likely have been selected for adaptations to changing salinity (Wurts, 1998). Likewise, prairie species such as the plains killifish and some copepod species exposed to drought conditions and the resulting alkaline environment also have adapted as a response, and may be more fit to survive increased alkalinity and conductivity (Nielson and others, 2003; Ostrand and Wilde, 2004).

Young organisms often are more sensitive than adults; however, the effects of toxicants on different species and lifestages of fish are variable (Lasier and others, 1997; U.S. Environmental Protection Agency, 2002; J.M. Besser, I.E. Greer, J.L. Kunz, C.G. Ingersoll, C.G., and N. Wang, written commun., 2004; Besser and others, 2005). In the present study, 2-dph and 4-dph FHM, and 22-dph and 69-dph white suckers were exposed to a range of $\mathrm{NaHCO}_{3}$ concentrations. In both experiments, the LC50s for younger fish and older fish, respectively, were 1,643 and approximately $4,000 \mathrm{mg}$ $\mathrm{NaHCO}_{3} / \mathrm{L}$ for FHM, and 5,121 and approximately 6,600 $\mathrm{mg} / \mathrm{L}$ for white suckers. U.S. Environmental Protection Agency (2002) guidelines require the use of the most sensitive lifestage for toxicity testing, and FHM must be 1-dph to 14-dph. However, because FHM more than 4-dph were less sensitive than 2-dph, experiments that use even slightly older fish may miss the most sensitive period of development for these fish. Therefore, it appears that with $\mathrm{NaHCO}_{3}$ toxicity, fish must be exposed immediately post hatch to insure that testing occurs during the most sensitive developmental period. Also, the effect of age on sensitivity was observed during field experiments in the present study completed in the Powder River Basin. In these experiments, a statistically significant difference in survival was observed between 2-dph and 6-dph fish, which further supports observations that very early lifestage (2-dph to 4-dph) are more sensitive than fish even just a few days older (see chapter 4).
In summary, the present study indicates that $\mathrm{NaHCO}_{3}$ is acutely toxic to freshwater organisms, including mussels, fish and C. dubia. The acute toxicity occurred within the range of concentrations defined by Mount and Gulley (1992) and Mount and others (1997), and are below concentrations measured in untreated CBNG produced water in the Powder River Basin (ALL Consulting, 2003; Patz and others, 2004). Additionally, the age at which fish are exposed to $\mathrm{NaHCO}_{3}$ appears to be inversely related to the severity of toxic responses. Therefore, assessments of $\mathrm{NaHCO}_{3}$ toxicity to fish should be initiated immediately after hatching to ensure that the most sensitive lifestage is used.

\section{References Cited}

ALL Consulting, 2003, Handbook on coalbed methane produced water-Management and beneficial use alternatives: coalbed natural gas resources and produced water management, Tulsa: ALL Consulting, Report to the U.S. Department of Energy, accessed April 2010 at http://www.all-llc. com/CBNG/BU/index.htm, $44 \mathrm{p}$.

American Society of Testing and Materials International, 1998, Standard guide for conducting the frog embryo teratagenesis assay-Xenopus (FETAX): West Conshohocken, Pa., American Society of Testing and Materials International, E1439-98, 16 p.

American Society of Testing and Materials International, 2006, Standard guide for conducting laboratory toxicity tests with freshwater mussels: West Conshohocken, Pa., American Society of Testing and Materials International, E 2455-06, $52 \mathrm{p}$.

Bantle, J.A., Dumont, J.N., Finch, R.A., and Linder, G., 1991, Atlas of abnormalities - a guide for the performance of FETAX: Stillwater, Oklahoma State University Publications, $63 \mathrm{p}$.

Besser, J.M., Wang, N., Dywer, F.J., Mayer, F.L., and Ingersoll, C.G., 2005, Assessing contaminant sensitivity of endangered and threatened aquatic species-Part I. Acute Toxicity of five chemicals chronic toxicity of copper and entachlorophenol to two endangered species and two surrogate species: Archives of Environmental Contamination and Toxicology, v. 48, p. 155-165.

Burnett, L.E., and McMahon, B.R., 1985, Facilitation of $\mathrm{CO}_{2}$ excretion by carbonic anhydrase located on the surface of the basal membrane of crab gill epithelium: Respiratory Physiology, v. 62, p. 341-348.

Burnham, B.L., and Peterka, J.J., 1975, Effects of saline water from North Dakota lakes on the survival of fathead minnows Pimephales promelas embryos and sac fry: Journal of Fisheries Research Board of Canada, v. 32, p. 809-812. 
Dickerson, K.K., Hubert, W.A., and Bergman, H.L., 1996, Toxicity assessment of water from lakes and wetlands receiving irrigation drain water: Environmental Toxicology and Chemistry, v. 15, no. 7, p. 1,097-1,101.

Dwyer, F.J., Mayer, F.L., Sappington, L.C., Buckler, D.R., Bridges, C.M., Greer, I.E., Hardesty, D.K., Henke, C.E., Ingersoll, C.G.,Kunz, J. L., Whites, D.W., Augspurger, T., Mount, D.R., Hattala, K., and Neuderfer, G.N., 2005, Assessing contaminant sensitivity of endangered and threatened aquatic species: Part I. acute toxicity of five chemicals: Archives of Environmental Contamination and Toxicology, v. 48 , p. $143-154$.

Gilmour, K.M., and Perry, S.F., 2009, Carbonic anhydrase and acid-base regulation in fish: Journal of Experimental Biology, v. 212, p. 1,647-1,661.

Haswell, M.S., Randall, D.J., and Perry, S.F., 1980, Fish gill carbonic anhydrase: acid base regulation or salt transport?: American Journal of Physiology, v. 238, p. 240-245.

Hobe, H., Wood, C.M., and McMahon, B., 1984, Mechanisms of acid/base and ionoregulation in white suckers Catostomus commersoni in natural soft water: Journal of Comparative Physiology, v. 154, p. 35-46.

Hoke, R.A., Gala, W.R., Drake, J.B., Giesy, J.P., and Flegler, S., 1992, Bicarbonate as a potential confounding factor in cladoceran toxicity assessments of pore water from contaminated sediments: Canadian Journal of Fisheries and Aquatic Sciences, v. 49, p. 1,633-1,640.

Lasier, P.J., Winger, P.V., and Reinert, R.E., 1997, Toxicity of alkalinity to Hyalella azteca: Bulletin of Environmental Contamination and Toxicology, v. 59, p. 807-814.

Montana Department of Fish Wildlife and Parks, 2010, Fatmucket—Lampsilis siliquoidea, Montana Field Guide: Montana Natural Heritage Program, accessed February 16, 2010, 2 p. at http://fieldguide.mt.gov/detail_IMBIV21280. aspx.

Mount, D.R., and Gulley, D.D., 1992, Development of a salinity toxicity relationship to predict acute toxicity of saline waters to freshwater organisms: Chicago, Gas Research Institute, GRI-92/0301, $10 \mathrm{p}$.

Mount, D.R., Gulley, D.D., Hockett, J.R., Garrison, T.D., and Evans, J.M., 1997, Statistical models to predict the toxicity of major ions to C. dubia, Daphnia magna and Pimephales promelas (fathead minnows): Evironmnetal Toxicology and Chemistry, v. 16, p., 2009-2019.

Nielsen, D.L., Brock, M.A., Rees, G.N., and Baldwin, D.S., 2003, Effects of increasing salinity on freshwater ecosystems in Australia: Australian Journal of Botany, v. 51, p. 655-665.
Ostrand, K.G., and Wilde, G.R., 2004, Changes in prairie stream fish assemblages restricted to isolated streambed pools: Transactions of the American Fisheries Society, v. 133 , p. 1,329-1,338.

Patz, M.J., Reddy, K.J., and Skinner, Q.D., 2004, Chemistry of coalbed methane discharge water interacting with semi-arid ephemeral stream channels: Journal of American Water Resources Association, v. 40, no. 5, p. 1,247-1,255.

Perry, S.F., 1997, The chloride cell: structure and function in the gills of freshwater fishes: Annual Review of Physiology, v. 59, p. $325-347$.

Rand, G.M., 1995, Fundamentals of Toxicology, 1: New York, Taylor and Francis, $125 \mathrm{p}$.

Randall, D J., and Tsui, T.J.N., 2006, Tribute to R.G. Boutilier-Acid-base transfer across fish gills: The Journal of Experimental Biology, v. 209, no. 1, p. 1179-1,184.

Sprague, J.B., 1973, The abc's of pollutant bioassays using fish in Cairns, J., and Dickson, J.L., eds., Biological methods for the assessment of water quality: Philadelphia, American Society of Testing and Materials International, p. 6-30.

Stephan, C.E., Mount, D.I., Hansen, D.J., Gentile, J.R., Chapman, G.A., and Brungs, W.A., 1985, Guidelines for deriving numerical national water quality criteria for the protection of aquatic organisms and their uses: Duluth, U.S. Environmental Protection Agency, Office of Research and Development, EPA-PB85-227049, 106 p.

Sullivan, G. V., Fryer, J.N., and Perry, S.F., 1995, Immunolocalization of proton pumps (H+-ATPase) in pavement cells of rainbow trout: Journal of Experimental Biology, v. 198, no. 2, p. 2,619-2,629.

Tietge, J.E., Hockett, J.R., and Evans, J.M., 1997, Major ion toxicity of six produced waters to three freshwater species: Application of ion toxicity models and TIE procedures. Environmental Toxicology and Chemistry, v. 16, no. 10, p. $2,002-2,008$.

U.S. Environmental Protection Agency, 2002, Methods for measuring the acute toxicity of effluents and receiving waters to freshwater and marine organisms, 5th edition: Washington, D. C., U.S. Environmental Protection Agency, Office of Water (4303T), EPA-821-R-02-012, 266 p.

U.S. Environmental Protection Agency, 2010, The Toxicity Relationship Analysis Program: Duluth, U.S. Environmental Protection Agency, Mid-Continent Ecology Division, 21 p. accessed April 25, 2011, at http://www.epa.gov/med/ Prods_Pubs/trap.htm. 
Wang, N., Ingersoll, C.G., Hardesty, D.K., Ivey, C.D., Kunz, J.L., May, T.W., Dwyer, F.J., Roberts, A.D., Augspurger, T. ,Kane, C.M., Neves, R.J., and Barnhart, M.C., 2007, Acute toxicity of copper, ammonia, chloride and chlorine to glochidea and juveniles of freshwater mussels (Unionidae): Evironmental Toxicology and Chemistry, v. 26, no. 2, p. 2,036-2,047.

Weber-Scannell, P.K., and Duffy, L., 2007, Effects of total dissolved solids on aquatic organisms: A review of literature and recommendation for salmonid species: American Journal of Environmental Science v. 3, no. 1, p. 1-6.
Wilson, J.M. and Laurent., P., 2002, Fish gill morphology; inside out: Journal of Experimental Zoology, v. 293, p. 192-213.

Wurts, W.A., 1998, Why can some fish live in fresh water, some in salt water, and some in both?: World Aquaculture, v. 29 , no. 1, p. 65 . 


\section{The Chronic Toxicity of Sodium Bicarbonate Defined Under Laboratory Conditions}

By Aïda M. Farag and David D. Harper

Chapter 3 of

The Potential Effects of Sodium Bicarbonate, a Major Constituent of Produced Waters from Coalbed Natural Gas Production, on Aquatic Life

Edited by Aïda M. Farag and David D. Harper

Prepared in cooperation with Montana Fish, Wildlife, and Parks, U.S. Bureau of Land Management, and the U.S. Environmental Protection Agency

Scientific Investigations Report 2012-5008 


\section{Contents}

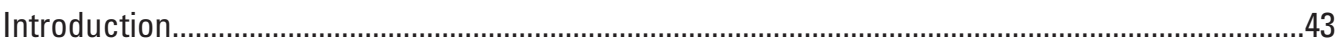

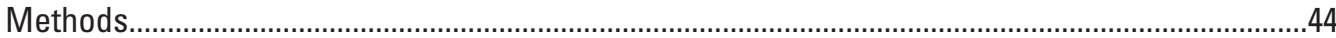

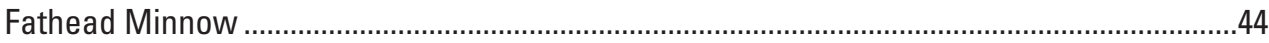

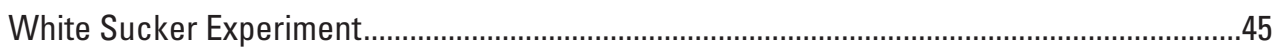

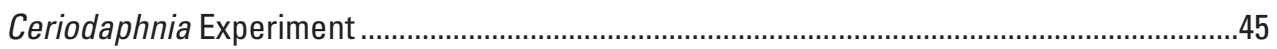

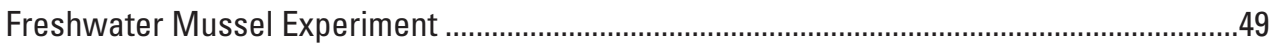

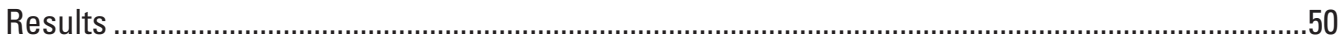

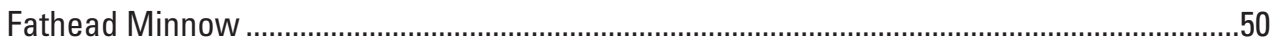

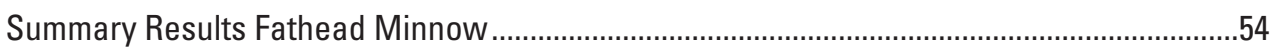

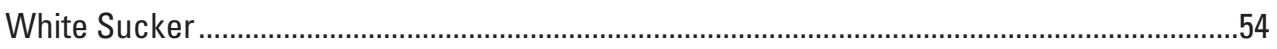

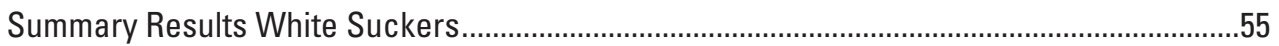

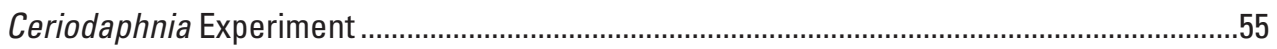

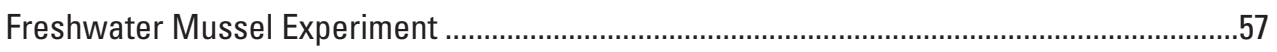

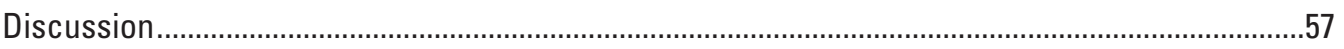

References Cited

\section{Tables}

3-1. Nominal exposure concentration of sodium bicarbonate, estimated number of eggs at the start of the experiment, number hatched and estimated percent hatch during chronic fathead minnow (Pimephales promelas), experiment 1 completed in reconsti-

tuted Tongue River water ....................................................................................................4

3-2. Mean temperature, dissolved oxygen, conductivity, $\mathrm{pH}$, alkalinity, bicarbonate, calcium, magnesium, sodium, potassium, chloride, and sulfate during chronic toxicity experiment 1 with fathead minnow (Pimephales promelas) completed in reconstituted Tongue River water.

3-3. Mean temperature, dissolved oxygen, conductivity, $\mathrm{pH}$, alkalinity, bicarbonate, and hardness, during chronic toxicity experiment 2 with fathead minnow (Pimephales promelas) completed in reconstituted Tongue River water

3-4. Mean alkalinity expressed as calcium carbonate equivalent, bicarbonate, hardness, $\mathrm{pH}$, conductivity, temperature, and dissolved oxygen during 7-day chronic toxicity experiment with fathead minnow (Pimephales promelas) less than 48-hours old.

3-5. Mean temperature, dissolved oxygen, conductivity, $\mathrm{pH}$, alkalinity, bicarbonate, calcium, magnesium, sodium, potassium, chloride, and sulfate during chronic toxicity experiment with white suckers (Catostomus commersoni) completed in reconstituted Tongue River water..

3-6. Water chemistry measured during a 7-day experiment with Ceriodaphnia (Ceriodaphnia dubia) completed in moderately hard reconstituted water. Exposures presented are nominal concentrations of sodium bicarbonate.

3-7. Water chemistry measured during a chronic static renewal experiment with fatmucket mussel (Lampsilis siliquoidea) completed in reconstituted Tongue River water

3-8. Percent survival of fathead minnows (Pimephales promelas) completed in reconstituted Tongue River water during chronic experiments 1 and 2. Exposures presented are nominal concentrations of sodium bicarbonate. 
3-9. Mean lengths and weights of fathead minnows (Pimephales promelas) at day 30 or 37 and day 60 of chronic experiments 1 and 2 completed in reconstituted Tongue River water

3-10. Mean sodium-potassium adenosine triphosphatase, $(\mathrm{N}+/ \mathrm{K}+$ ATPase), also known as the $\mathrm{Na}+/ \mathrm{K}+$ pump, activity in micromoles adenosine diphosphate per milligram protein per hour in whole body fathead minnows (Pimephales promelas) at day 30 or 37 and day 60 during experiments 1 and 2

3-11. Mean whole body ions in fathead minnows (Pimephales promelas) during experiment 1 completed in reconstituted Tongue River water with nominal concentrations of sodium bicarbonate.

3-12. Mean whole body ions in milligrams per gram dry weight in fathead minnows (Pimephales promelas) on day 30 of chronic experiment 2 completed in reconstituted Tongue River water.

3-13. Mean percent survival during a 7-day static renewal chronic exposure of fathead minnow (Pimephales promelas) completed in reconstituted Tongue River water

3-14. Mean lengths of fathead minnows (Pimephales promelas) at day 7 of a 7-day static renewal exposure completed in reconstituted Tongue River water.

3-15. Mean percent survival of white suckers (Catostomus commersoni) during a 60-day (53-day post-hatch) chronic exposure completed in reconstituted Tongue River water......

3-16. Mean percent survival of white suckers (Catostomus commersoni) during a 60-day (53-day post-hatch) chronic exposure completed in reconstituted Tongue River water.

3-17. Mean whole-body ions in white suckers (Catostomus commersoni) on day 30 of a chronic experiment completed in reconstituted Tongue River water

3-18. Mean whole-body ions in white suckers (Catostomus commersoni) on day 53 of a chronic experiment completed in reconstituted Tongue River water.

3-19. Survival and reproduction of Ceriodaphnia dubia exposed to nominal concentrations of milligrams sodium bicarbonate per liter $\left(\mathrm{mg} \mathrm{NaHCO}_{3} / \mathrm{L}\right)$ in moderately hard reconstituted water......

3-20. Survival of 10-day old newly transformed fatmucket mussels (Lampsilis siliquoidea) to chronic exposures completed in reconstituted Tongue River water. 



\title{
The Chronic Toxicity of Sodium Bicarbonate Defined Under Laboratory Conditions
}

\author{
By Aïda M. Farag and David D. Harper
}

\section{Introduction}

As a result of surface discharge, ephemeral streams in the arid Powder River Basin may consist of 100 percent Coalbed Natural Gas (CBNG ) produced water effluent during certain seasons. Thus, aquatic life inhabiting newly created perennial stream habitats may be exposed continuously to undiluted effluent water throughout the year. Sodium bicarbonate $\left(\mathrm{NaHCO}_{3}\right)$ is the principle salt in CBNG produced water discharged at many locations in the Powder River Basin, and individual measured concentrations of up to 3,000 milligrams sodium bicarbonate per liter $(\mathrm{mg} \mathrm{NaHCO} / \mathrm{L})$ have been documented at some locations (ALL Consulting, 2003; Patz and others, 2004). Whereas acutely toxic effects of $\mathrm{NaHCO}_{3}$ have been investigated (Mount and others 1997, Chapter 2 in this report), no adequate studies have been performed to assess the potential chronic effects of $\mathrm{NaHCO}_{3}$ exposure. The U.S. Environmental Protection Agency (USEPA) guidelines for the establishment of water-quality criteria for the protection of aquatic life suggest that a minimum of three chronic experiments be completed with one fish species, one invertebrate species, and one acutely sensitive freshwater species (Stephan and others, 1985). Specific species selected for chronic toxicity studies should be easily maintained in a laboratory facility for extended periods of time; should be identical to species used in acute toxicity experiments for comparisons of acute and chronic toxicity; and should be representative of species that are found in the region of concern (for example, Tongue and Powder River Basins of Montana and Wyoming) for regional applicability. However, the use of species with a range outside of the local basin could provide useful information for managers throughout the western United States and elsewhere that encounter elevated concentrations of $\mathrm{NaHCO}_{3}$.

In addition to population level effects (for example, decreased survival), sublethal effects at the individual organism level may be evaluated to provide information about the mechanisms of toxicity related to elevated concentrations of $\mathrm{NaHCO}_{3}$. Sodium bicarbonate may disassociate in the water column into separate ions of sodium $\left(\mathrm{Na}^{+}\right)$and bicarbonate $\left(\mathrm{HCO}_{3}^{-}\right)$; two ions that play a key role in acid/base balance and concomitant ionoregulation in freshwater organisms.
The ionoregulatory status of young fish can be assessed by measuring whole-body ions (Reid and MacDonald, 1988). Additionally, the enzyme sodium-potassium adenosine triphosphatase, $\left(\mathrm{N}^{+} / \mathrm{K}^{+}\right.$ATPase), also known as the $\mathrm{Na}^{+} / \mathrm{K}^{+}$pump, is responsible for $\mathrm{Na}^{+}$movement across the basal membrane of gills and other organs into the blood (Marshall, 2002). The measurement of $\mathrm{N}^{+} / \mathrm{K}^{+}$ATPase concentrations during $\mathrm{NaHCO}_{3}$ exposure could explain a mechanism by which a change in ion content within the animal takes place. Moreover, histological examinations may provide additional information related to the ionoregulatory status of aquatic organisms. For example, increases in the number of mitochondrial rich cells has been observed when fish are exposed to brackish water, and it is these types of cells that are considered responsible for ion and acid/base regulation in freshwater organisms (Wilson and Laurent, 2002). Studies designed to include measurements of the above endpoints may provide explanations of the mechanisms of action that affect survival or growth observed and would support the definition of an effect concentration.

The present study was initiated to assess the chronic toxicity of $\mathrm{NaHCO}_{3}$ to aquatic organisms. The objectives of the study were to (1) define the chronic effects of $\mathrm{NaHCO}_{3}$, a principle component of CBNG production, on aquatic life; (2) to define sublethal effects at the individual organism level to explain the mechanisms of toxicity during chronic exposure; and (3) to define the effect concentrations in relation to concentrations measured in the field so that managers could be more aware of the extent that $\mathrm{NaHCO}_{3}$ may or may not currently affect aquatic life in the Tongue and Powder River Basins of Montana and Wyoming. Multiple aquatic species were used in the assessment of the chronic toxicity of $\mathrm{NaHCO}_{3}$, which allows for sensitivity comparisons among species and improves the level of confidence in observed responses. The exposure of representative species from different phyla, such as fish and aquatic invertebrates, further enhances the confidence in experimental results by expanding the possible range of potential effects. Further, the inclusion of an experimental species that is known to be acutely sensitive to $\mathrm{NaHCO}_{3}$ defines concentrations of $\mathrm{NaHCO}_{3}$ that would protect some of the most sensitive species, a goal of established water-quality criteria. 


\section{Methods}

Chronic toxicity experiments were completed with four aquatic species to meet the strategy for the selection of test species described above. Three chronic experiments were completed with fathead minnows (Pimephales promelas, FHM), one with white suckers (Catostomus commersoni), one with Ceriodaphnia dubia, and one with fatmuckets (a freshwater mussel, Lampsilis siliquoidea). All of these experiments could be paired with the acute toxicity experiments completed in the present study for comparison of effect concentrations and to derive acute/chronic ratios as suggested by the USEPA (Stephan and others, 1985; see chapter 6). All four species were maintained in the laboratory for extended periods of time, though white sucker had to be spawned in the field. The four experimental species are resident in the Tongue and Powder River Basins, and are found throughout the United States. Sublethal parameters were monitored during two of the FHM experiments, and to a lesser extent, during the white sucker experiment.

The FHM (during two of the three experiments) and white suckers were exposed to $\mathrm{NaHCO}_{3}$ in a flow-through diluter system (Mount and Brungs, 1967). The diluter system contained 12, 4-L glass exposure chambers that were replicated 3-4 times and supplied with 250 milliliters $(\mathrm{mL})$ of reconstituted Tongue River (as prepared in chapter 2) water every 15 minutes to obtain the desired exposure concentrations. Complete water replacement in each chamber occurred approximately every 4 hours (h).

\section{Fathead Minnow}

Two flow-through chronic toxicity experiments were completed with FHMs exposed to $\mathrm{NaHCO}_{3}$. The FHMs were exposed to concentrations that ranged from 500 to 1,400 milligrams sodium bicarbonate per liter $\left(\mathrm{mg} \mathrm{NaHCO}_{3} / \mathrm{L}\right)$ in experiment 1 and from 300 to $625 \mathrm{mg} \mathrm{NaHCO} / \mathrm{L}$ in experiment 2. Also, fish were held in a control treatment of reconstituted Tongue River water without added $\mathrm{NaHCO}_{3}$. Both experiments were terminated at 60-days-post-hatch (referred to from here as dph), a 65-day (d) total exposure.

Before initiating the experiment, the FHM eggs were carefully picked from spawning tiles and combined into a 2-L, acid-washed, plastic container of reconstituted Tongue River water. Seventy eggs from this container were randomly pipetted into each replicate experimental chamber. This method was used to ensure that each replicated exposure chamber contained eggs from several different females. Steps were taken to minimize inaccuracies of egg counts (for example, manual counters used to physically document eggs as they entered the chamber, multiple counts performed by separate researchers on an identical chamber); however, the small size of individual FHM eggs suggests that an exact number of eggs might be difficult to assess. Therefore, the calculated percent hatch for control and experimental concentrations was an estimate.
Though not tested statistically because these were estimates, percentage hatch of the eggs at $1,400 \mathrm{mg} \mathrm{NaHCO} / \mathrm{L}_{3}$ were smaller compared to the controls during experiment 1 (table 3-1). Percent hatch ranged from 44 to 78 percent. Fish were fed a ration of 10 cultured brine shrimp/fish twice per day. This ration was doubled as fish grew.

Table 3-1. Nominal exposure concentration of sodium bicarbonate, estimated number of eggs at the start of the experiment, number hatched and estimated percent hatch during chronic fathead minnow (Pimephales promelas), experiment 1 completed in reconstituted Tongue River water (control). Exposures presented are nominal concentrations of sodium bicarbonate.

[ $\mathrm{NaHCO}_{3}$, sodium bicarbonate; $\mathrm{mg} / \mathrm{L}$, milligrams per liter]

\begin{tabular}{cccc}
\hline $\begin{array}{c}\text { Exposure } \\
\text { (mg NaHCO} / \mathbf{L})\end{array}$ & $\begin{array}{c}\text { Estimated } \\
\text { number of eggs } \\
\text { added }\end{array}$ & $\begin{array}{c}\text { Number of } \\
\text { eggs hatched }\end{array}$ & $\begin{array}{c}\text { Esimated } \\
\text { percent } \\
\text { hatched }\end{array}$ \\
\hline Control & 280 & 175 & 62.5 \\
\hline 500 & 280 & 219 & 78.2 \\
\hline 800 & 280 & 194 & 69.3 \\
\hline 1,100 & 280 & 197 & 70.4 \\
\hline 1,400 & 280 & 123 & 43.9 \\
\hline
\end{tabular}

Survival, conductivity, dissolved oxygen (DO), and temperature were monitored daily in each replicate during the experiments. The $\mathrm{pH}$, hardness, and alkalinity were measured weekly from alternate replicates so that all were sampled at least three times throughout the experiment. Also, water samples were collected weekly (from systematically selected replicates with a random starting point) in 150-mL certified clean I-Chem ${ }^{\circledR}$ vials and shipped to Red Buttes Environmental Laboratory in Laramie, Wyoming for analyses of major ions, including calcium $(\mathrm{Ca})$, potassium $(\mathrm{K})$, sodium $(\mathrm{Na})$, and magnesium (Mg), along with Quality Assurance and Quality Control (QA/QC) samples that were measured with an atomic absorption spectrophotometer (table 3-2, 3-3). The measured concentrations of $\mathrm{mg} \mathrm{NaHCO} / \mathrm{L}$ were within \pm 10 percent of the nominal concentration and the nominal concentrations will be used throughout this report. There was a drop in the $\mathrm{Ca}$ concentration measured in the water as the amount of $\mathrm{mg}$ $\mathrm{NaHCO}_{3} / \mathrm{L}$ increased. Calcium in the water decreased 21 percent in the $500 \mathrm{mg} \mathrm{NaHCO}_{3} / \mathrm{L}$ treatment to 53 percent in the $1,400 \mathrm{mg} \mathrm{NaHCO} / \mathrm{L}_{3}$ compared to the control. In fact, white particulate matter was observed on aquaria and metering devices used for the larger concentrations during the experiment.

At 37-days post median hatch (30-days for experiment 2 ), about 50 percent of the fish remaining in each replicate exposure chamber were randomly sampled. The lengths and weights of these fish were measured and then the whole fish were placed in vials with Davidson's solution added to 10 times the volume of tissue, for histological examinations. At 60 days of exposure, the remaining fish were collected, 
weighed, measured for length, and placed in appropriate vials for histological analyses.

Samples for histological analyses were shipped to the U.S. Fish and Wildlife Service in Bozeman, Montana, not shown, and examined by a procedure similar to Farag and others (2003).

All anion/cation analyses were accompanied by standard QA/QC analyses and were all within acceptable limits. The anion/cation charge balance ranged from 0.35 percent to 4.67 percent difference and the percent recovery of $\mathrm{Na}$ in each treatment ranged from 94 percent to 104 percent.

A 7-d FHM static-renewal chronic experiment also was conducted with reconstituted Tongue River water (water reconstituted with same procedure described in chapter 2). Fathead minnows less than $(<)$ 48-h old were received from Aquatic BioSystems (Ft. Collins, Colorado) and acclimated to increasing concentrations of reconstituted Tongue River (control) water for a 1-h time period. Five hundred $\mathrm{mL}$ of control and concentrations of 300 to $2,000 \mathrm{mg} \mathrm{NaHCO} / \mathrm{L}$ water were added to 1-L chambers and held in a water bath that was maintained at 25 degrees Celsius $\left({ }^{\circ} \mathrm{C}\right) \pm 1$. The experiment was initiated by adding 10 fish to each chamber in a nonsystematic order. Fish were fed 200 microliters $(\mu \mathrm{L})$ of a brine shrimp suspension twice daily.

Survival was monitored daily. The experimental waters were replaced daily and conductivity, DO, and temperature were monitored in a composite of the discarded replicate waters. The $\mathrm{pH}$, hardness, and alkalinity were measured from alternate replicates so that all were sampled at least three times throughout the 7-d experiment. A variable $\mathrm{pH}$ alkalinity measurement was performed where the sample was titrated to $\mathrm{pH} 8.3$ and $\mathrm{pH} 4.5$ to provide $\mathrm{HCO}_{3}{ }^{-} \mathrm{mg} / \mathrm{L}$ measurements in addition to total alkalinity as milligrams calcium carbonate per liter ( $\mathrm{mg} \mathrm{CaCO}_{3} / \mathrm{L}$ ) (table 3-4; American Public Health Association, 1975).

\section{White Sucker Experiment}

The white suckers for the experiment were obtained from a private pond near Dillon, Montana, not shown. The artificial pond receives water from the Beaverhead River, not shown, by way of an irrigation ditch. The pond water quality consisted of a $\mathrm{pH}$ of 8.69 , total alkalinity of $136 \mathrm{mg} / \mathrm{L}$ as $\mathrm{CaCO}_{3}$ and a specific conductance of 416 microsiemens per centimeter $(\mu \mathrm{S} /$ $\mathrm{cm})$. Sexually mature white suckers were captured in trap nets that were set overnight on multiple occasions. Eggs from three females were fertilized with sperm from seven males in reconstituted Tongue River water. The fertilized eggs were treated in a solution of $4.7 \mathrm{~mL}$ of iodine dissolved in $2 \mathrm{~L}$ of reconstituted Tongue River water for 30 minutes. The eggs were then rinsed and transported to the U.S. Geological Survey, Columbia Environmental Research Center, Jackson Field Research Station (from here defined as Jackson Field Research Station) in a plastic cooler filled with reconstituted Tongue River water.
Upon arrival at the Jackson Field Research Station the eggs were acclimated from $16^{\circ} \mathrm{C}$ to $18^{\circ} \mathrm{C}$ and approximately 50-60 eggs were placed in each of the 4-L chambers in the flow-through diluter system described in the second paragraph of the methods section above. The white suckers were exposed for 63-d (51-dph) to 450, 800, and 1,400 $\mathrm{mg} \mathrm{NaHCO}_{3} / \mathrm{L}$ and a control with no added $\mathrm{NaHCO}_{3}$. Each exposure concentration was replicated three times and randomly assigned across the 12 glass aquaria in the diluter. After 30 days of exposure, fish were collected from each replicate experimental chamber and the number of surviving fish was reduced to $<15$ fish per 4-L replicate chamber. The sampled fish were anesthetized with ice, measured for length and weight, and prepared for histological and whole-body ion analyses. Fish were fed a commercial diet at 8 percent body weight per day that was split among four rations.

Conductivity, DO, and temperature were monitored daily in each replicate. Alkalinity, hardness, and $\mathrm{pH}$ were measured in a sample collected from each batch of prepared simulated Tongue River water and were measured two times in each concentration. Water samples were collected six times from each concentration for analyses of total $\mathrm{NaHCO}_{3}$. This procedure and chemistry sampling was similar to that used during the fathead minnow experiments (table 3-5). The measured concentrations of $\mathrm{mg} \mathrm{NaHCO} / \mathrm{L}$ were within \pm 13 percent of the nominal concentration and the nominal concentrations will be used throughout the remaining text.

\section{Ceriodaphina Experiment}

Ceriodaphnia dubia were exposed in a static-renewal system to various concentrations of $\mathrm{NaHCO}_{3}$ until 60 percent of the surviving individuals produced three broods of offspring. The experiments were completed according to the USEPA protocol EPA/600/4-91/002 (U.S. Environmental Protection Agency, 1994). Brood stock C. dubia were obtained from Aquatic BioSystems, Fort Collins, Colorado. Neonates from individual brood stock cultures were used in the experiments. Individuals were cultured in $30-\mathrm{mL}$ plastic cups containing $15 \mathrm{~mL}$ of reconstituted, moderately hard water maintained at $25^{\circ} \mathrm{C}$. Individuals were fed a combination of yeast, CEROPHYLL ${ }^{\circledR}$, and Trout chow (YCT), and the unicellular green algae, Selenastrum capricornutum daily. Neonates from individual cultures that produced at least 20 offspring were used for the experiments.

Experimental concentrations of $\mathrm{NaHCO}_{3}$ were obtained by adding the appropriate quantities of $\mathrm{NaHCO}_{3}$ to reconstituted moderately hard culture water. Acute toxicity rangefinding experiments were completed to determine the concentrations used in the chronic toxicity experiment. The nominal concentrations used were 500, 700, 900, 1,200, and $1,500 \mathrm{mg} \mathrm{NaHCO} / \mathrm{L}$ (table 3-6) plus a control of moderately hard reconstituted water with no added $\mathrm{NaHCO}_{3}$. Ten C. dubia were placed individually into replicate $30-\mathrm{mL}$ plastic exposure containers with $15 \mathrm{~mL}$ of experimental water. The exposure 


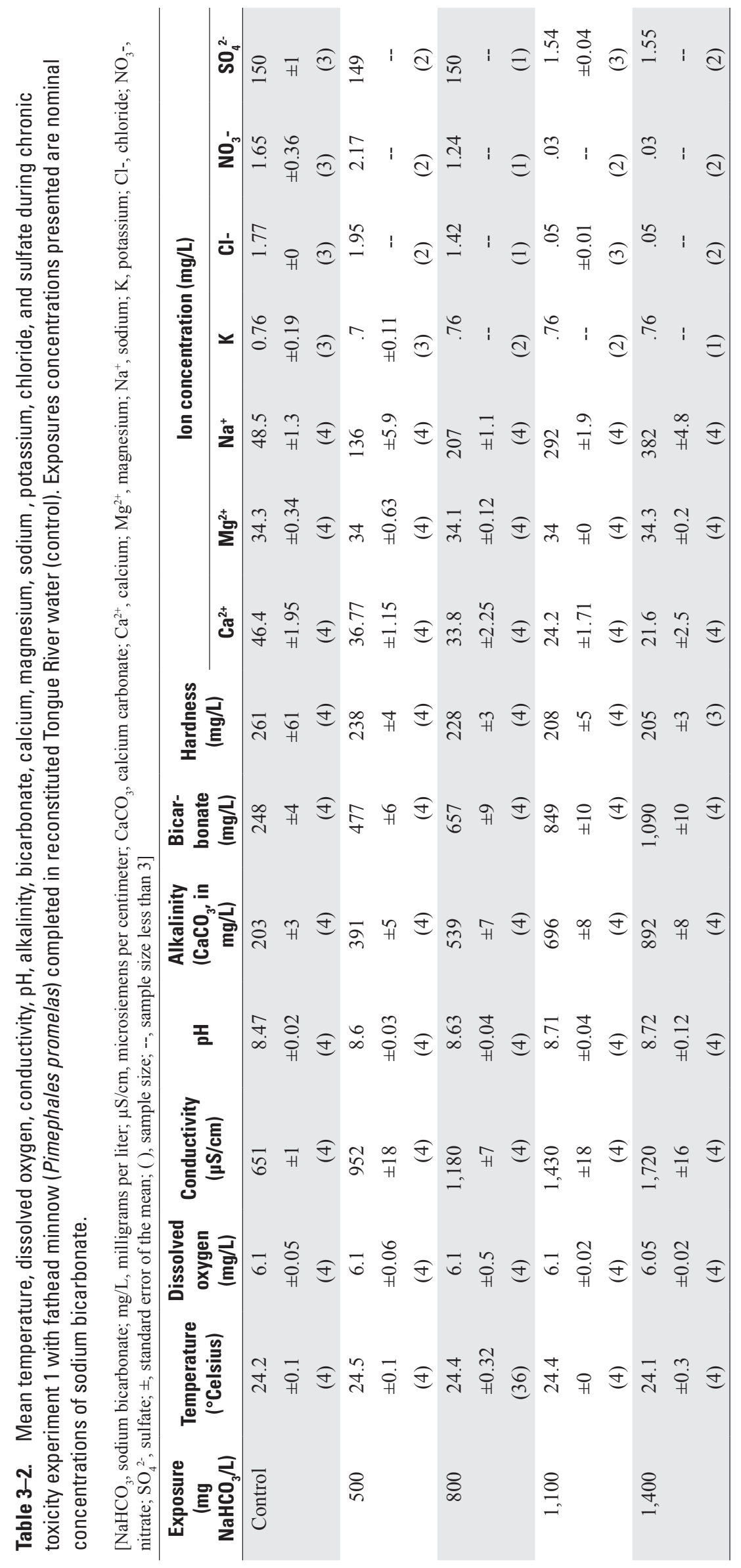


Table 3-3. Mean temperature, dissolved oxygen, conductivity, $\mathrm{pH}$, alkalinity, bicarbonate, and hardness, during chronic toxicity experiment 2 with fathead minnow (Pimephales promelas) completed in reconstituted Tongue River water (control). Exposures presented are nominal concentrations of sodium bicarbonate.

$\left[\mathrm{NaHCO}_{3}\right.$, sodium bicarbonate; $\mathrm{mg} / \mathrm{L}$, milligrams per liter; $\mu \mathrm{S} / \mathrm{cm}$, microsiemens per centimeter; $\mathrm{CaCO}_{3}$, calcium carbonate; \pm , standard error of the mean; ( ), number of samples]

\begin{tabular}{cccccccc}
\hline $\begin{array}{c}\text { Exposure } \\
\mathbf{( m g}\end{array}$ & $\begin{array}{c}\text { Temperature } \\
\left.\mathbf{~}^{\circ} \text { Celsius }\right)\end{array}$ & $\begin{array}{c}\text { Dissolved } \\
\mathbf{0 x y g e n} \\
(\mathbf{m g} / \mathbf{L})\end{array}$ & $\begin{array}{c}\text { Conductivity } \\
(\boldsymbol{\mu} \mathbf{S} / \mathbf{c m})\end{array}$ & $\mathbf{p H}$ & $\begin{array}{c}\text { Alkalinity } \\
\left(\mathbf{C a C O}_{\mathbf{3}^{\prime}} \text { in }\right. \\
\mathbf{m g} / \mathbf{L})\end{array}$ & $\begin{array}{c}\text { Bicar- } \\
\text { bonate } \\
(\mathbf{m g} / \mathbf{L})\end{array}$ & $\begin{array}{c}\text { Hardness } \\
(\mathbf{m g} / \mathbf{L})\end{array}$ \\
\hline Control & 25.0 & 6.35 & 652 & 8.51 & 200 & 316 & 259 \\
& \pm 0.2 & \pm 0.34 & \pm 5 & \pm 0.12 & \pm 6.2 & \pm 7.1 & \pm 5.8 \\
& $(61)$ & $(61)$ & $(61)$ & $(4)$ & $(4)$ & $(4)$ & $(4)$ \\
300 & 25.0 & 6.49 & 773 & 8.51 & 277 & 311 & 255 \\
& \pm 0.2 & \pm 0.30 & \pm 8 & \pm 0.09 & \pm 14 & \pm 8.5 & \pm 7 \\
& $(61)$ & $(61)$ & $(61)$ & $(4)$ & $(4)$ & $(4)$ & $(4)$ \\
400 & 25.0 & 6.36 & 847 & 8.53 & 320 & 306 & 251 \\
& \pm 0.2 & \pm 0.30 & \pm 45 & \pm 0.06 & \pm 13 & \pm 5 & \pm 4 \\
& $(61)$ & $(61)$ & $(61)$ & $(4)$ & $(4)$ & $(4)$ & $(4)$ \\
625 & 25.0 & 6.52 & 1,040 & 8.55 & 437 & 295 & 242 \\
& \pm 0.3 & \pm 0.30 & \pm 11 & \pm 0.05 & \pm 19 & \pm 7.3 & \pm 6 \\
& $(61)$ & $(61)$ & $(61)$ & $(4)$ & $(4)$ & $(4)$ & $(4)$ \\
\hline
\end{tabular}

Table 3-4. Mean alkalinity expressed as calcium carbonate equivalent, bicarbonate, hardness, $\mathrm{pH}$, conductivity, temperature, and dissolved oxygen during 7-day chronic toxicity experiment with fathead minnows (Pimephales promelas) less than 48-hours old. Completed in reconstituted Tongue River water (control). Exposures presented are nominal concentrations of sodium bicarbonate. Sample size is three for each.

$\left[\mathrm{NaHCO}_{3}\right.$, sodium bicarbonate; $\mathrm{CaCO}_{3}$, calcium carbonate; $\mu \mathrm{S} / \mathrm{cm}$, microsiemens per centimeter; \pm , standard error of the mean $]$

\begin{tabular}{|c|c|c|c|c|c|c|c|}
\hline $\begin{array}{l}\text { Exposure } \\
\text { (mg } \\
\left.\mathrm{NaHCO}_{3} / \mathrm{L}\right)\end{array}$ & $\begin{array}{c}\text { Alkalinity } \\
\left(\mathrm{CaCO}_{3^{\prime}} \text { in }\right. \\
\mathrm{mg} / \mathrm{L})\end{array}$ & $\begin{array}{l}\text { Bicarbonate } \\
\text { (mg/L) }\end{array}$ & $\begin{array}{c}\text { Hardness } \\
\text { (mg/L) }\end{array}$ & $\mathrm{pH}$ & $\begin{array}{c}\text { Conductivity } \\
\text { ( } \mu \mathrm{S} / \mathrm{cm})\end{array}$ & $\begin{array}{c}\text { Temperature } \\
\left({ }^{\circ} \text { Celsius }\right)\end{array}$ & $\begin{array}{c}\text { Dissolved } \\
\text { oxygen } \\
\text { (mg/L) }\end{array}$ \\
\hline \multirow[t]{2}{*}{ Control } & 193 & 241 & 253 & 8.4 & 631 & 24.5 & 5.7 \\
\hline & \pm 7 & \pm 18 & \pm 8.1 & \pm 0.01 & \pm 5.2 & \pm 0.1 & \pm 0.2 \\
\hline \multirow[t]{2}{*}{300} & 273 & 335 & 260 & 8.5 & 754 & 24.5 & 5.7 \\
\hline & \pm 3 & \pm 3 & \pm 4 & \pm .02 & \pm 4 & \pm .07 & \pm .1 \\
\hline \multirow[t]{2}{*}{500} & 378 & 446 & 233 & 8.6 & 914 & 24.5 & 5.8 \\
\hline & \pm 2 & \pm 8 & \pm 5.8 & \pm .003 & \pm 14.9 & \pm .09 & \pm .1 \\
\hline \multirow[t]{2}{*}{625} & 445 & 519 & 226 & 8.6 & 1,030 & 24.5 & 5.5 \\
\hline & \pm 0.8 & \pm 8 & \pm 12.5 & \pm .02 & \pm 3.6 & \pm .2 & \pm .2 \\
\hline \multirow[t]{2}{*}{800} & 544 & 636 & 205 & 8.6 & 1,180 & 24.6 & 5.63 \\
\hline & \pm 4 & \pm 8 & \pm 14.5 & \pm .07 & \pm 2.8 & \pm .02 & \pm .3 \\
\hline \multirow[t]{2}{*}{1,100} & 685 & 792 & 176 & 8.6 & 1,430 & 24.6 & 5.6 \\
\hline & \pm 7 & \pm 3 & \pm 6.9 & \pm .05 & \pm 44.5 & \pm .01 & \pm .1 \\
\hline \multirow[t]{2}{*}{1,400} & 867 & 985 & 165 & 8.6 & 1,700 & 24.6 & 5.6 \\
\hline & \pm 67 & \pm 5 & \pm 7 & \pm .02 & \pm 2.3 & \pm .07 & \pm .09 \\
\hline \multirow[t]{2}{*}{2,000} & 1,160 & 1,349 & 148 & 8.8 & 2,200 & 24.6 & 5.6 \\
\hline & \pm 3 & \pm 8 & \pm 2 & \pm .01 & \pm .9 & \pm .03 & \pm .1 \\
\hline
\end{tabular}




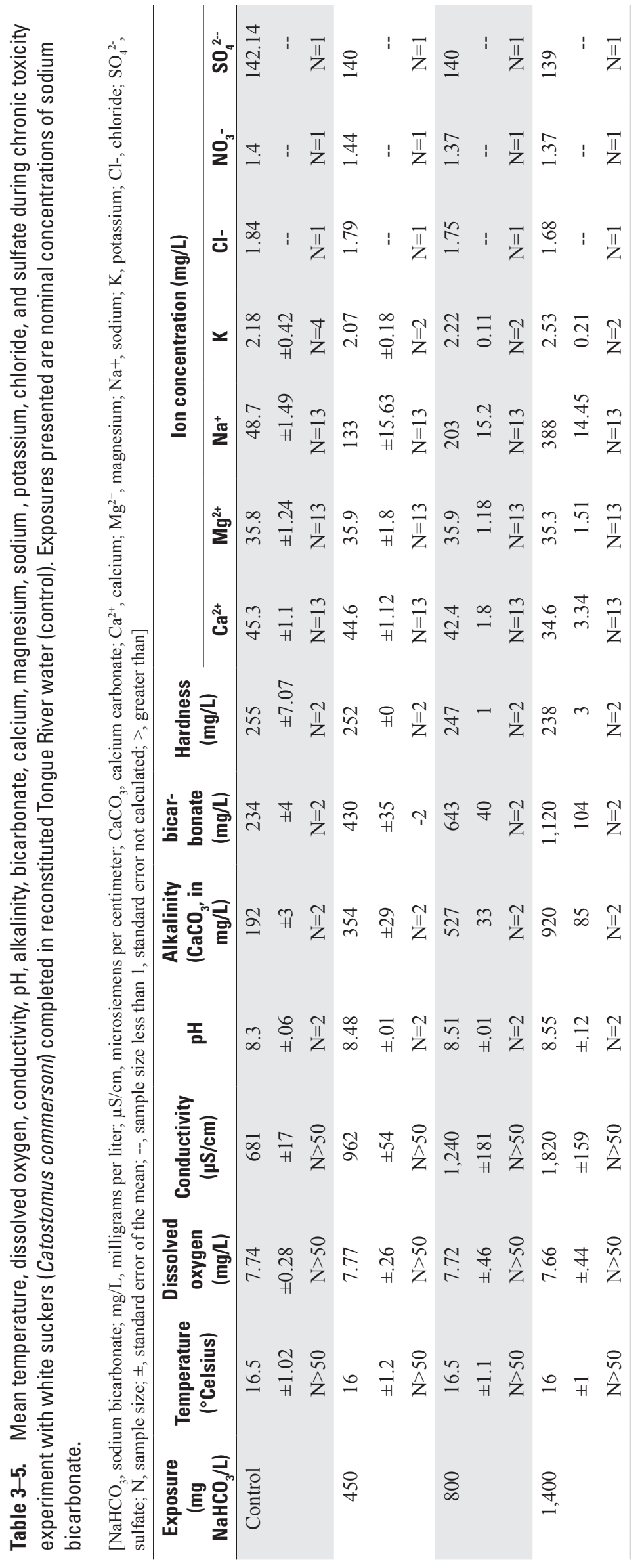


Table 3-6. Water chemistry measured during a 7-day experiment with Ceriodaphnia (Ceriodaphnia dubia) completed in moderately hard reconstituted water (control). Exposures presented are nominal concentrations of sodium bicarbonate.

$\left[\mathrm{NaHCO}_{3}\right.$, sodium bicarbonate; $\mathrm{mg} / \mathrm{L}$, milligrams per liter; $\mathrm{K}$, potassium; $\mathrm{CaCO}_{3}$, calcium carbonate; $\mathrm{Ca}^{2+}$, calcium; $\mathrm{Mg}^{2+}$, magnesium; $\mathrm{Na}^{+}$, sodium]

\begin{tabular}{|c|c|c|c|c|c|c|c|}
\hline \multirow{2}{*}{$\begin{array}{l}\text { Exposure } \\
\text { (mg } \\
\left.\mathrm{NaHCO}_{3} / \mathrm{L}\right)\end{array}$} & \multirow{2}{*}{$\begin{array}{c}\text { Alkalinity } \\
\left(\mathrm{CaCO}_{3^{\prime}} \text { in }\right. \\
\mathrm{mg} / \mathrm{L})\end{array}$} & \multirow{2}{*}{$\begin{array}{c}\text { Bicarbonate } \\
\text { (mg/L) }\end{array}$} & \multicolumn{5}{|c|}{ Ion concentration (mg/L) } \\
\hline & & & $\mathrm{Ca}^{2+}$ & $\mathbf{M g}^{2+}$ & $\mathrm{Na}^{+}$ & $\mathbf{K}$ & Sulfate \\
\hline Control & 76 & 93 & 15 & 9 & 22 & 8 & 96 \\
\hline 500 & 319 & 345 & 15 & 13 & 121 & 3 & 97 \\
\hline 700 & 410 & 446 & 15 & 13 & 182 & 3 & 97 \\
\hline 900 & 546 & 579 & 14 & 13 & 216 & 3 & 99 \\
\hline 1,200 & 721 & 750 & 15 & 12 & 339 & 2 & 97 \\
\hline 1,500 & 911 & 938 & 14 & 12 & 390 & 3 & 100 \\
\hline
\end{tabular}

containers were held in a water bath at $25 \pm 1^{\circ} \mathrm{C}$. The experiment lasted for 7 to 8 days and the $C$. dubia were fed YCT and algal suspension daily. The water in each replicate experimental container was replaced daily, and the number of surviving adult $C$. dubia and the number of neonates produced were recorded daily.

The 3 criteria for a successful experiment based on responses observed in the control treatment were; 80 percent or more survival of females, an average of 15 or more young produced per surviving female, and the production of 3 broods by 60 percent of surviving control organisms. The exposure period could be extended to 8 days to allow for individuals to produce three broods.

\section{Freshwater Mussel Experiment}

Advances during the last 10 years in culture and testing methods development with freshwater mussels led to the establishment of American Society of Testing and Materials International guidelines for conducting acute and chronic experiments with freshwater fatmucket mussel, Lampsilis siliquoidea (American Society of Testing and Materials
International 2006; Wang and others, 2007). Newly transformed juvenile fatmucket mussels were obtained from Missouri State University, Springfield, Missouri, USA. The juvenile mussels were held in a water bath that contained well water maintained at $20^{\circ} \mathrm{C}$ (range was $20.0-22.8^{\circ} \mathrm{C}$ ) at the Jackson Field Research Station. Before starting the experiment, mussels were fed a mixture of commercially prepared algae and shellfish diets during a 48-h acclimatization period. Five mussels were randomly placed into each of four replicate 30-mL beakers per exposure concentration. The mussels were exposed to 500, 750, 1,250, 1,500, and 2,000 $\mathrm{mg} \mathrm{NaHCO}_{3} / \mathrm{L}$ and a control of reconstituted Tongue River water in a 10-d static renewal experiment according to the procedures described by Wang and others (2007) and American Society of Testing and Materials International (2006). The mussels were fed twice daily during the experiment. Water from each concentration was sampled at the beginning and end of the experiment and analyzed as described above (table 3-7).

The Spearman-Karber method was used to calculate LC50s at 96-h and 37-d with TOXSTAT 3.4. Significant differences for various endpoints for the 60-d FHM and white sucker experiments were defined by a one-way ANOVA

Table 3-7. Water chemistry measured during a chronic static renewal experiment with fatmucket mussel (Lampsilis siliquoidea) completed in reconstituted Tongue River water (control). Exposures presented are nominal concentrations of sodium bicarbonate. Carbonate, bicarbonate, pH, calcium, magnesium, sodium, potassium, chloride, nitrate, and sulfate provided. Sample size equals 2.

$\left[\mathrm{NaHCO}_{3}\right.$, sodium bicarbonate; $\mathrm{mg} / \mathrm{L}$, milligrams per liter; $\mathrm{Ca}^{2+}$, calcium, $\mathrm{Mg}^{2+}$, magnesium,; $\mathrm{Na}^{+}$, sodium; $\mathrm{K}$, potassium; $\mathrm{Cl}^{-}$, chloride; $\mathrm{NO}_{3}^{-}$, nitrate; $\mathrm{SO}_{4}^{2-}$, sulfate]

\begin{tabular}{|c|c|c|c|c|c|c|c|c|c|c|c|}
\hline $\begin{array}{l}\text { Exposure } \\
\text { (mg } \\
\left.\mathrm{NaHCO}_{3} / \mathrm{L}\right)\end{array}$ & $\begin{array}{c}\text { Alkalinity } \\
\left(\mathrm{CaCO}_{3} \text {, in }\right. \\
\mathrm{mg} / \mathrm{L})\end{array}$ & $\begin{array}{c}\text { Carbonate } \\
\left(\mathrm{CaCO}_{3^{\prime}} \text { in }\right. \\
\mathrm{mg} / \mathrm{L})\end{array}$ & $\begin{array}{c}\text { Bicarbonate } \\
\text { (mg/L) }\end{array}$ & pH & \multicolumn{7}{|c|}{ Ion concentration (mg/L) } \\
\hline Control & 204 & 11 & 235 & 8.72 & 48 & 34 & 46 & 2.2 & 2 & 0.3 & 150 \\
\hline 500 & 367 & 26 & 416 & 8.62 & 37 & 34 & 129 & 2.2 & 2 & .2 & 148 \\
\hline 750 & 511 & 1 & 623 & 8.59 & 22 & 33 & 189 & 2.2 & 1.4 & .5 & 152 \\
\hline 1,000 & 672 & 8 & 810 & 8.76 & 20 & 33 & 255 & 2.2 & 1 & .5 & 151 \\
\hline 1,500 & 934 & 33 & 1,099 & 8.84 & 16 & 33 & 367 & 2.1 & 1.2 & .4 & 150 \\
\hline
\end{tabular}


followed by either a Dunnett's post hoc means comparison for survival or Tukey means comparison for $\mathrm{N}^{+} / \mathrm{K}^{+}$ATPase and whole-body ion concentrations. Data analyses were performed according to the methodology described in U.S. Environmental Protection Agency protocol EPA/600/4-91/002 (1994). The Toxicity Relationship Analysis Program (TRAP) (U.S. Environmental Protection Agency, 2010) was used to calculate the Inhibition Concentration (IC) that affects 20 percent of the sample population (IC20) based on mortality. The Linear Interpolation Method (Norberg-King 1993) was used to define IC20 based on growth or reproduction. Survival data for the 7-d experiment were analyzed with the TRAP (U.S. Environmental Protection Agency, 2010) to determine the LC50 and the 95-percent Confidence Interval (CI). The IC20 calculated as $\mathrm{mg} \mathrm{NaHCO}_{3} / \mathrm{L}$ were based on measured rather than nominal concentrations, except for the 7-d FHM experiment. The $\mathrm{Na}$ measurements for the 7-d FHM experiment were not available at the time of printing. Many of the IC20 also are reported as $\mathrm{mg} \mathrm{HCO}_{3}^{-} / \mathrm{L}$ and total alkalinity (expressed as $\mathrm{mg} \mathrm{CaCO}_{3} / \mathrm{L}$ ). The Trimmed Spearman-Karber Program was used to calculate a 10-d effect concentration on 50 percent of the sample population (EC50).

\section{Results}

\section{Fathead Minnow}

A 60-d LC50 could not be calculated because of the elevated mortality that occurred in the $800,1,100$, and 1,400 $\mathrm{mg} \mathrm{NaHCO} / \mathrm{L}$ exposure concentrations by day 37 . The FHM 96-h [ \pm standard deviation (SD)] and 37-d LC50s were $1002+$ $94 \mathrm{mg} \mathrm{NaHCO} / \mathrm{L}$ and $877+71 \mathrm{mg} \mathrm{NaHCO} / \mathrm{L}$, respectively. During experiment 1 , FHM survival decreased significantly at concentrations $>500 \mathrm{mg} \mathrm{NaHCO}_{3} / \mathrm{L}$ at day 37 (table 3-8). In experiment 2, FHM survival decreased significantly in the 625 $\mathrm{mg} \mathrm{NaHCO} / \mathrm{L}$ concentration at day 30 (table 3-8). It should be noted that survival in the control from 0-30 days was 68 percent. This is 2 percent below the guideline of 70 percent for a successful early lifestage experiment. Freshly fertilized eggs were used to start this experiment and the mortalities observed in the control happened on days 4-6. Significant mortalities were observed in $500 \mathrm{mg} \mathrm{NaHCO} / / \mathrm{L}$ during experiment 1 and in $625 \mathrm{mg} \mathrm{NaHCO} / \mathrm{L}$ during experiment 2 . Because the observed effects bracket those observed during experiment 1 when survival in the control was 89 percent, experiment 2 data continued to be used for interpretation. However, an IC20 for FHM cumulative $37-\mathrm{d}$ survival was $462 \mathrm{mg} \mathrm{NaHCO} / \mathrm{L}$ with a 95-percent $\mathrm{CI}$ of $278-645 \mathrm{mg} \mathrm{NaHCO} / \mathrm{L}$ and was calculated with data from experiment 1 only. This also can be expressed as $368 \mathrm{mg} \mathrm{HCO}_{3}^{-} / \mathrm{L}$ (95-percent $\mathrm{CI}, 239-497 \mathrm{HCO}_{3}^{-} / \mathrm{L}$ ), and

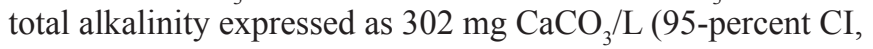
$196-408 \mathrm{CaCO}_{3} / \mathrm{L}$ ) An IC20 based on survival was not calculated for experiment 2.

No significant differences were observed among treatment groups for weights and lengths (table 3-9). Because a T-test revealed no significant difference between lengths and weights of control fish at day 37 from experiment 1 and day 30 from experiment 2 , the data were combined for a pooled reference. Although no significant difference for growth was observed as a result of exposure, a significant increase in length and weight was observed at day 60 for the pooled reference when compared to those at days 30 and 37 , and these reference data indicate the fish grew appropriately for the course of the experiments. An IC20 could not be calculated based on growth for FHM from experiment 1 and 2.

Significant decreases in $\mathrm{Na} / \mathrm{K}$ ATPase were observed during the FHM experiment 1 . At day 37, exposures to $\geq 625 \mathrm{mg} \mathrm{NaHCO} / \mathrm{L}$ resulted in significant $\mathrm{Na} / \mathrm{K}$ ATPase decreases compared to the controls (table 3-10). The decreases were not completely concentration dependent because the $800 \mathrm{mg} \mathrm{NaHCO} / /$ L resulted in less of a decrease than the $625 \mathrm{mg} \mathrm{NaHCO} / /$ L exposure. On day 60 , all the $\mathrm{NaHCO}_{3}$ treatments were associated with a significant decrease in $\mathrm{Na} / \mathrm{K}$ ATPase and the amount of decrease appeared to be concentration dependent. It should be noted that the sample number $\mathrm{N}=4$ for all treatments except the $625 \mathrm{mg} \mathrm{NaHCO} / \mathrm{L}$, where $\mathrm{N}=2$. Therefore, data were analyzed additionally with the Bonferroni test and the results were similar. However, the reader is cautioned about the small sample size for the $625 \mathrm{mg}$ $\mathrm{NaHCO}_{3} / \mathrm{L}$ treatment.

Table 3-8. Percent survival of fathead minnows (Pimephales promelas) completed in reconstituted Tongue River water (control) during chronic experiments 1 and 2. Exposures presented are nominal concentrations of sodium bicarbonate. Note: egg mortality from "estimated percent hatch" reported in table 3-1 is not included in the percent survival calculations.

$\left[\mathrm{NaHCO}_{3}\right.$, sodium bicarbonate; $\mathrm{mg} / \mathrm{L}$, milligrams per liter; +, sacrificed concentrations at day 37 ; --, no concentration exposed or data gathered]

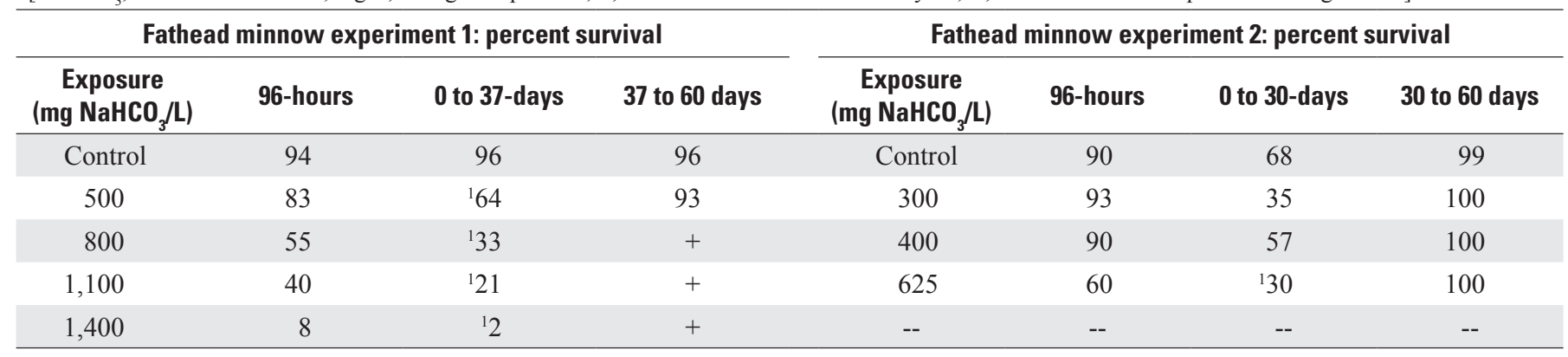

${ }^{1}$ Significant difference at $\alpha=0.05$. 
There were significant increases in whole-body $\mathrm{Ca}$ and $\mathrm{Mg}$ during both FHM experiments (tables 3-11, 3-12). Whole-body Ca was elevated in FHM exposed to 500 and $625 \mathrm{mg} \mathrm{NaHCO} / \mathrm{L}$ treatments. Although not significant, a trend of elevated $\mathrm{Ca}$ was observed in the 800 and $1,100 \mathrm{mg}$ $\mathrm{NaHCO}_{3} / \mathrm{L}$ treatments. Also, whole-body $\mathrm{Mg}$ was elevated in fish from the $625 \mathrm{mg} \mathrm{NaHCO} / \mathrm{L}$ on days 30 and 60 , and whole-body $\mathrm{Na}$ was elevated in fish exposed to $1,100 \mathrm{mg}$ $\mathrm{NaHCO}_{3} / \mathrm{L}$. The increase in whole-body $\mathrm{Ca}$ and $\mathrm{Mg}$ may be associated with the noted drop in measured $\mathrm{Ca}$ in the water column (table 3-2).

It should be noted that, as was the case with $\mathrm{Na} / \mathrm{K}$ ATPase measurements, $\mathrm{N}=2$ for the $625 \mathrm{mg} \mathrm{NaHCO} / / \mathrm{L}$ treatment. The Bonferroni test was used again; however, the repeatability of these trends lends confidence to the statistical interpretation regardless of the small $\mathrm{N}$ for this one treatment.

Rodlet cells were found closely associated with cranial meninges in all groups of fish and appeared to increase with concentrations or the number of days of exposure. Similarly, focal degeneration in ovarian tissue appeared to be more common in fish from the greater $\mathrm{NaHCO}_{3}$ exposure concentrations, but no degenerative changes of this type were observed in fish from the control and $500 \mathrm{mg} \mathrm{NaHCO}_{3} / \mathrm{L}$ treatments.

Gill lesions (epithelial hypertrophy, edema, mucus and necrosis) increased in severity with $\mathrm{NaHCO}_{3}$ concentration or the number of days of exposure. For example, at $30 \mathrm{~d}$ of exposure, fish from the control exhibited no gill necrosis, whereas fish from the $1,100 \mathrm{mg} \mathrm{NaHCO} / \mathrm{L}_{3}$ exposure experienced moderate changes and contained focal areas of necrosis in the gills. At $60 \mathrm{~d}$, large necrotic lesions were observed in the gills of fish exposed to the control and $500 \mathrm{mg} \mathrm{NaHCO} / \mathrm{L}$ treatment, but were more numerous in the $500 \mathrm{mg} / \mathrm{L} \mathrm{NaHCO}_{3}$ treatment. Changes were not observed in the kidney at day 30 , but at day 60 the numbers of regenerating tubules were slightly greater in fish exposed to the $500 \mathrm{mg} \mathrm{NaHCO} / \mathrm{L}$ treatment compared to those in the controls. There were no notations about mitochondrial rich cells during the histological examinations and the presence of necrotic cells in the gills may have precluded observations of these types of cells. Because swelling was present, the small size of these tissues also may preclude the observation of mitochondrial rich cells.

For additional comparisons, a third experiment, a 7-d FHM experiment was included. This experiment was not initiated with eggs, but instead was initiated with $<2$-dph FHM. Survival of FHM during the 7-d static renewal experiment was reduced significantly at concentrations $>1,100 \mathrm{mg} \mathrm{NaHCO}_{3} / \mathrm{L}$ compared to the control (table 3-13). The 7-d LC50 is 1,857 mg NaHCO$/ 2$ (95-percent CI, 1,507-2,108 mg NaHCO$/ \mathrm{L}$ ). An IC20 based on survival at 7-d was calculated as 1,190 mg $\mathrm{NaHCO}_{3} / \mathrm{L}$ (95-percent CI, 536-643 mg NaHCO$/ \mathrm{L}$ ). The IC20 also may be expressed as $903 \mathrm{mg} \mathrm{HCO}_{3}^{-} / \mathrm{L}$ (95-percent CI, 803-1,003 $\left.\mathrm{mg} \mathrm{NaHCO}_{3} / \mathrm{L}\right)$ and total alkalinity expressed as $780 \mathrm{mg} \mathrm{CaCO} / \mathrm{L}$ (95-percent CI, 693-866 mg $\mathrm{NaHCO}_{3} / \mathrm{L}$ ). Weights of FHM were reduced significantly at $>625 \mathrm{mg} \mathrm{NaHCO} / \mathrm{L}_{3}$ compared to the control (table 3-14). An IC20 based on growth was calculated as $591 \mathrm{mg} \mathrm{NaHCO} / \mathrm{L}$ (95-percent CI, 536-643 mg $\mathrm{NaHCO}_{3} / \mathrm{L}$ ). This can also be expressed as $499 \mathrm{mg} / \mathrm{L} \mathrm{HCO}_{3}{ }^{-/} / \mathrm{L}$ (95-percent CI, 467-531) or 424 (95-percent CI, 397-454) total alkalinity expressed as mg $\mathrm{CaCO}_{3} / \mathrm{L}$.

Table 3-9. Mean lengths and weights of fathead minnows (Pimephales promelas) at day 30 or 37 and day 60 of chronic experiments 1 and 2 completed in reconstituted Tongue River water (control 1 and control 2). Exposures presented are nominal concentrations sodium bicarbonate. Sample size (N) equals four unless indicated otherwise.

$\left[\mathrm{NaHCO}_{3}\right.$, sodium bicarbonate; mg/L, milligrams per liter; mm, millimeter; mg, milligram; ( ), standard deviation; NS, no sample; --, no calculation of standard deviation]

\begin{tabular}{|c|c|c|c|c|}
\hline $\begin{array}{c}\text { Exposure } \\
\text { (mg NaHCO} / \mathrm{L})\end{array}$ & $\begin{array}{l}\text { Length (mm) } \\
\text { day } 30 \text { or } 37\end{array}$ & $\begin{array}{l}\text { Weight (mg) } \\
\text { day } 30 \text { or } 37\end{array}$ & $\begin{array}{c}\text { Length }(\mathrm{mm}) \\
\text { day } \mathbf{6 0}\end{array}$ & $\begin{array}{c}\text { Weight (mg) } \\
\text { day } 60\end{array}$ \\
\hline \multirow[t]{2}{*}{ Control 1} & 14.93 & 0.03 & 25.75 & 0.18 \\
\hline & $(.7)$ & $(.01)$ & (1.13) & $(.06)$ \\
\hline \multirow[t]{2}{*}{ Control 2} & 15.2 & .03 & 25.81 & .16 \\
\hline & $(.37)$ & 0 & $(.7)$ & $(.01)$ \\
\hline \multirow[t]{2}{*}{300} & $15.88, \mathrm{~N}=3$ & $.04, \mathrm{~N}=3$ & 28.34 & .22 \\
\hline & (1) & $(.01)$ & $(3.02)$ & $(.07)$ \\
\hline \multirow[t]{2}{*}{400} & $15.44, \mathrm{~N}=3$ & .03 & 26.2 & .18 \\
\hline & $(0.13)$ & 0 & $(1.45)$ & $(.03)$ \\
\hline \multirow[t]{2}{*}{500} & 15.44 & .04 & 25.45 & .19 \\
\hline & $(2.28)$ & $(.02)$ & (2.9) & $(.06)$ \\
\hline \multirow[t]{2}{*}{625} & $15.05, \mathrm{~N}=2$ & $.03, \mathrm{~N}=2$ & $24.68, \mathrm{~N}=2$ & $.16, \mathrm{~N}=2$ \\
\hline & $(.19)$ & 0 & $(.11)$ & $(.01)$ \\
\hline \multirow[t]{2}{*}{800} & 15.26 & .04 & NS & NS \\
\hline & (1.68) & $(.02)$ & -- & -- \\
\hline \multirow[t]{2}{*}{1,100} & 15.32 & .03 & NS & NS \\
\hline & $(1.49)$ & $(.01)$ & -- & -- \\
\hline
\end{tabular}


Table 3-10. Mean sodium-potassium adenosine triphosphatase, $\left(\mathrm{N}^{+} / \mathrm{K}^{+}\right.$ATPase), also known as the $\mathrm{Na}^{+} / \mathrm{K}^{+}$pump, activity in micromoles adenosine diphosphate per milligram protein per hour in whole body fathead minnows (Pimephales promelas) at day 30 or 37 and day 60 during experiments 1 and 2. Completed in reconstituted Tongue River water (control) with nominal concentrations of bicarbonate.

$\left[\mathrm{NaHCO}_{3}\right.$, sodium bicarbonate; $\mathrm{mg} / \mathrm{L}$, milligrams per liter; $\mu$ moles ADP/mg protein $/ \mathrm{hr}$, micromoles adenosine diphosphate per milligram protein per hour; ( ), standard deviation; NS, no sample; --, no calculation of standard deviation]

\begin{tabular}{|c|c|c|c|c|}
\hline $\begin{array}{c}\text { Exposure } \\
(\mathrm{mg} \mathrm{NaHCO} / \mathrm{L})\end{array}$ & Sample size & $\begin{array}{c}\text { Activity day } 30 \text { or } 37 \\
\text { ( } 1 \text { moles ADP/mg } \\
\text { protein/hr) }\end{array}$ & Sample size & $\begin{array}{c}\text { Activity day } 60 \\
\text { ( } 1 \text { moles ADP/mg } \\
\text { protein/hr) }\end{array}$ \\
\hline \multirow[t]{2}{*}{ Control } & 8 & $13.63^{\mathrm{a}}$ & 8 & $22.08^{\mathrm{a}}$ \\
\hline & & $(.67)$ & & $(.45)$ \\
\hline \multirow[t]{2}{*}{300} & 3 & $12.49^{\mathrm{a}}$ & 4 & $19.32^{\mathrm{b}}$ \\
\hline & & (1.13) & & $(.18)$ \\
\hline \multirow[t]{2}{*}{400} & 3 & $10.40^{\mathrm{a}}$ & 4 & $17.90^{b, c}$ \\
\hline & & $(0.49)$ & & $(.29)$ \\
\hline \multirow[t]{2}{*}{500} & 4 & $11.59^{\mathrm{a}}$ & 4 & $16.22^{\mathrm{c}, \mathrm{d}}$ \\
\hline & & $(.96)$ & & $(.42)$ \\
\hline \multirow[t]{2}{*}{625} & 2 & $4.80^{b}$ & 2 & $12.26^{\mathrm{d}}$ \\
\hline & & $(.66)$ & & $(.59)$ \\
\hline \multirow[t]{2}{*}{800} & 4 & $7.37^{c}$ & -- & NS \\
\hline & & $(.80)$ & -- & -- \\
\hline \multirow[t]{2}{*}{1,100} & 4 & $3.00^{\mathrm{b}}$ & -- & NS \\
\hline & & $(.42)$ & -- & -- \\
\hline
\end{tabular}

a,b,c Different superscript letters denote significant differences $(\alpha=0.05)$.

Table 3-11. Mean whole body ions in fathead minnows (Pimephales promelas) during experiment 1 completed in reconstituted Tongue River water (control) with nominal concentrations of sodium bicarbonate. Sample size equals 4 for all exposures except $800 \mathrm{mg} \mathrm{NaHCO} / \mathrm{L}$ where sample size equals three. Exposures presented are nominal concentrations of sodium bicarbonate

$\left[\mathrm{NaHCO}_{3}\right.$, sodium bicarbonate; $\mathrm{mg} / \mathrm{L}$, milligrams per liter; ( ), \pm standard error of the mean]

\begin{tabular}{|c|c|c|c|c|}
\hline $\begin{array}{c}\text { Exposure } \\
\text { (mg NaHCO} / \mathrm{L})\end{array}$ & $\begin{array}{l}\text { Calcium } \\
\text { (mg/L) }\end{array}$ & $\begin{array}{l}\text { Magnesium } \\
\text { (mg/L) }\end{array}$ & $\begin{array}{c}\text { Potassium } \\
\text { (mg/L) }\end{array}$ & $\begin{array}{c}\text { Sodium } \\
\text { (mg/L) }\end{array}$ \\
\hline \multicolumn{5}{|c|}{ Day 37} \\
\hline \multirow[t]{2}{*}{ Control } & $36.86^{\mathrm{a}}$ & $2.98^{\mathrm{a}}$ & 17.23 & $6.32^{\mathrm{a}}$ \\
\hline & $(2.01)$ & $(0.16)$ & $(0.82)$ & $(0.39)$ \\
\hline \multirow[t]{2}{*}{500} & $65.95^{\mathrm{b}}$ & $3.90^{\mathrm{b}}$ & 15.72 & $6.50^{\mathrm{a}}$ \\
\hline & $(6.29)$ & $(0.35)$ & $(0.42)$ & $(0.14)$ \\
\hline \multirow[t]{2}{*}{800} & $53.91^{\mathrm{a}, \mathrm{b}}$ & $3.07^{\mathrm{a}, \mathrm{b}}$ & 17.26 & $7.66^{\mathrm{a}, \mathrm{b}}$ \\
\hline & (4.9) & $(0.07)$ & $(0.51$ & $(0.37)$ \\
\hline \multirow[t]{2}{*}{1,100} & $43.28^{\mathrm{a}, \mathrm{b}}$ & $2.92^{\mathrm{a}, \mathrm{b}}$ & 17.76 & $8.83^{\mathrm{b}}$ \\
\hline & $(0.9)$ & $(0.05)$ & $(0.33)$ & $(0.39)$ \\
\hline \multicolumn{5}{|c|}{ Day 60} \\
\hline \multirow[t]{2}{*}{ Control } & $25.33^{\mathrm{a}}$ & $2.20^{\mathrm{a}}$ & 13.27 & 9.67 \\
\hline & $(2.36)$ & $(0.15)$ & $(0.51)$ & $(0.36)$ \\
\hline \multirow[t]{2}{*}{500} & $54.02^{\mathrm{b}}$ & $3.30^{\mathrm{b}}$ & 12.08 & 8.9 \\
\hline & $(7.21)$ & $(0.36)$ & $(0.29)$ & $(0.33)$ \\
\hline
\end{tabular}

${ }^{\mathrm{a}, \mathrm{b}}$ Different superscript letters denote significant differences $(\alpha=0.05)$. 
Table 3-12. Mean whole body ions in milligrams per gram dry weight $(\mathrm{mg} / \mathrm{g})$ in fathead minnows (Pimephales promelas) on day 30 of chronic experiment 2 completed in reconstituted Tongue River water (control). Exposures presented are nominal concentrations of sodium bicarbonate. Standard error of the mean is in parenthesis. Sample size equals four for control, three for 300 and $400 \mathrm{mg}$ $\mathrm{NaHCO}_{3} / \mathrm{L}$ and two for $625 \mathrm{mg} \mathrm{NaHCO} / \mathrm{L}$.

[ $\mathrm{NaHCO}_{3}$, sodium bicarbonate; $\mathrm{mg} / \mathrm{L}$, milligrams per liter; ( ), standard error of the mean]

\begin{tabular}{ccccc}
\hline $\begin{array}{c}\text { Exposure } \\
\text { (mg NaHCO} \mathbf{3} / \mathbf{L})\end{array}$ & $\begin{array}{c}\text { Calcium } \\
(\mathbf{m g} / \mathbf{L})\end{array}$ & $\begin{array}{c}\text { Magnesium } \\
(\mathbf{m g} / \mathbf{L})\end{array}$ & $\begin{array}{c}\text { Potassium } \\
(\mathbf{m g} / \mathbf{L})\end{array}$ & $\begin{array}{c}\text { Sodium } \\
(\mathbf{m g} / \mathbf{L})\end{array}$ \\
\hline & & Day 37 & \\
\hline Control & $21.27^{\mathrm{a}}$ & $2.20^{\mathrm{a}}$ & 11.92 & 9.18 \\
& $(3.22)$ & $(.13)$ & $(.4)$ & $(.27)$ \\
300 & $22.13^{\mathrm{a}}$ & $2.14^{\mathrm{a}}$ & 11.92 & 9.1 \\
& $(1.92)$ & $(.05)$ & $(.23)$ & $(.27)$ \\
400 & $28.91^{\mathrm{a}, \mathrm{b}}$ & $2.23^{\mathrm{a}}$ & 12.6 & 9.63 \\
& $(2.47)$ & $(.05)$ & $(.33)$ & $(.31)$ \\
& $42.26^{\mathrm{b}}$ & $2.86^{\mathrm{b}}$ & 12.29 & 8.87 \\
& $(11.99)$ & $(.22)$ & $(.57)$ & $(.35)$ \\
\hline
\end{tabular}

${ }^{\mathrm{a}, \mathrm{b}}$ Superscript letters denote significant differences $(\alpha=0.05)$.

Table 3-13. Mean percent survival during a 7-day static renewal chronic exposure of fathead minnow (Pimephales promelas) completed in reconstituted Tongue River water (control). Exposure concentrations presented are nominal values of sodium bicarbonate. Sample size equals three.

$\left[\mathrm{NaHCO}_{3}\right.$, sodium bicarbonate; $\mathrm{mg} / \mathrm{L}$, milligrams per; ( ), standard deviation; --, not calculated]

\begin{tabular}{|c|c|c|}
\hline $\begin{array}{c}\text { Exposure } \\
\left(\mathrm{mg} \mathrm{NaHCO}_{3} / \mathrm{L}\right)\end{array}$ & $\begin{array}{l}\text { 7-day mean num- } \\
\text { ber of individuals }\end{array}$ & Percent survival \\
\hline \multirow[t]{2}{*}{ Control } & 10 & 100 \\
\hline & (0) & -- \\
\hline \multirow[t]{2}{*}{300} & 10 & 100 \\
\hline & $(0)$ & -- \\
\hline \multirow[t]{2}{*}{500} & 10 & 100 \\
\hline & $(0)$ & -- \\
\hline \multirow[t]{2}{*}{625} & 9.3 & 93 \\
\hline & (.6) & -- \\
\hline \multirow[t]{2}{*}{800} & 10 & 100 \\
\hline & (0) & -- \\
\hline \multirow[t]{2}{*}{1,100} & 17.7 & 77 \\
\hline & $(2.3)$ & -- \\
\hline \multirow[t]{2}{*}{1,400} & 17.7 & 77 \\
\hline & $(1.5)$ & -- \\
\hline \multirow[t]{2}{*}{2,000} & 14.3 & 43 \\
\hline & $(1.2)$ & -- \\
\hline
\end{tabular}

${ }^{1}$ Significant difference at $\alpha=0.05$.
Table 3-14. Mean lengths of fathead minnows (Pimephales promelas) at day 7 of a 7-day static renewal exposure completed in reconstituted Tongue River water (control). Exposure concentrations presented are nominal values of sodium bicarbonate. Sample size equals three.

$\left[\mathrm{NaHCO}_{3}\right.$, sodium bicarbonate; $\mathrm{mg} / \mathrm{L}$, milligrams per liter; mg, milligrams; ( ), standard deviation]

\begin{tabular}{cc}
\hline $\begin{array}{c}\text { Exposure } \\
\left(\mathbf{m g ~ N a H C O}_{3} / \mathbf{L}\right)\end{array}$ & Day 30 weight $(\mathbf{m g})$ \\
\hline Control & $(.0005)$ \\
\hline 300 & .006 \\
& $(.001)$ \\
500 & .005 \\
& $(.0006)$ \\
625 & ${ }^{1} .004$ \\
& $(.0004$ \\
800 & ${ }^{1} .004$ \\
& $(.00007)$ \\
1,100 & ${ }^{1} .004$ \\
& $(.0008)$ \\
1,400 & ${ }^{1} .003$ \\
& $(.0006)$ \\
2,000 & .003 \\
& $(.0009)$ \\
\hline
\end{tabular}

${ }^{1}$ Significant difference compared to the control $(\alpha=0.05)$. 


\section{Summary Results Fathead Minnow}

During the flow-through chronic experiments, the survival of FHM was significantly less than the control in all concentrations $\geq 500 \mathrm{mg} / \mathrm{L} \mathrm{NaHCO}_{3}$. In the 300 and $400 \mathrm{mg}$ $\mathrm{NaHCO}_{3} / \mathrm{L}$ treatments that did not reduce survival, growth was not affected. $\mathrm{Na} / \mathrm{K}$ ATPase decreased in all treatments $>625 \mathrm{mg} \mathrm{NaHCO} / \mathrm{L}$ at day 37 and was decreased in all treatments in a concentration dependent manner by day 60 . The severity of the microscopic lesions was related directly to $\mathrm{NaHCO}_{3}$ concentration and the duration of exposure. After $60 \mathrm{~d}$, fish from the $500 \mathrm{mg} \mathrm{NaHCO}_{3} / \mathrm{L}$ treatment exhibited a slight increase in the incidence of kidney damage, including a greater number of regenerating tubules and focal degeneration, compared to fish in the control treatment. Similarly, the severity of gill lesions was related directly to $\mathrm{NaHCO}_{3}$ exposure concentration and duration of exposure. Whereas significant mortality was not observed in the 7-d static renewal experiment until $>1,100 \mathrm{mg} \mathrm{NaHCO} / 3$, weights were reduced in FHM from this experiment in concentrations $>625 \mathrm{mg}$ $\mathrm{NaHCO}_{3} / \mathrm{L}$.

\section{White Sucker}

Estimated percent hatch of white sucker eggs was 23 percent in the control, 21 percent in the $450 \mathrm{mg} / \mathrm{L} \mathrm{NaHCO}_{3}$ treatment, 29 percent in the $800 \mathrm{mg} / \mathrm{L} \mathrm{NaHCO}$ treatment, and 30 percent in the $1,400 \mathrm{mg} / \mathrm{L} \mathrm{NaHCO}_{3}$ treatment. After 30 days, the survival of white sucker fry that hatched was 54 percent in the control, 80 percent in the $450 \mathrm{mg} / \mathrm{L} \mathrm{NaHCO}_{3}$ exposure, 60 percent in $800 \mathrm{mg} / \mathrm{L} \mathrm{NaHCO}_{3}$ exposure, and 51 percent in the $1,400 \mathrm{mg} / \mathrm{L} \mathrm{NaHCO}_{3}$ exposure. From 31-60-d survival was 70-82 percent in the control and

Table 3-15. Mean percent survival of white suckers (Catostomus commersoni) during a 60-day (53-day posthatch) chronic exposure completed in reconstituted Tongue River water (control). Exposure concentrations presented are nominal values of sodium bicarbonate. Standard deviation is plus or minus $( \pm)$ mean in parentheses and sample size equals three.

$\left[\mathrm{NaHCO}_{3}\right.$, sodium bicarbonate; $\mathrm{mg} / \mathrm{L}$, milligrams per $]$

\begin{tabular}{ccc}
\hline $\begin{array}{c}\text { Exposure } \\
\text { (mg NaHCO} / \mathbf{L})\end{array}$ & Day 0-30 & Day 31-60 \\
\hline Control & 54 & 70 \\
& $( \pm 5.0)$ & $( \pm 15)$ \\
450 & 80 & 72 \\
& $( \pm 3)$ & $( \pm 20)$ \\
800 & 60 & 82 \\
& $( \pm 17)$ & $( \pm 7.7)$ \\
1,400 & 51 & 45 \\
& $( \pm 13)$ & $( \pm 29)$ \\
\hline
\end{tabular}

Table 3-16. Mean lengths and weights of white suckers (Catostomus commersoni) during a 60-day (53-day posthatch) chronic exposure completed in reconstituted Tongue River water (control). Exposure concentrations presented are nominal values of sodium bicarbonate. Sample size equals three.

$\left[\mathrm{NaHCO}_{3}\right.$, sodium bicarbonate; $\mathrm{mg} / \mathrm{L}$, milligrams per liter; $\mathrm{mm}$, millimeters; mg, milligrams; ( ), standard deviation]

\begin{tabular}{ccc}
\hline $\begin{array}{c}\text { Exposure } \\
\left(\mathbf{m g ~ N a H C O}_{\mathbf{3}} / \mathbf{L}\right)\end{array}$ & $\begin{array}{c}\text { Length } \\
(\mathbf{m m})\end{array}$ & $\begin{array}{c}\text { Weight } \\
(\mathbf{m g})\end{array}$ \\
\hline Control & 19.9 & 0.04 \\
& $(.4)$ & $(.004)$ \\
450 & ${ }^{1} 17.6$ & ${ }^{1} 0.024$ \\
& $(1.1)$ & $(.004)$ \\
\multirow{2}{*}{800} & ${ }^{1} 17.4$ & 10.022 \\
& $(1)$ & $(.05)$ \\
1,400 & 16.4 & 10.019 \\
& $(.4)$ & $(.002)$ \\
\hline
\end{tabular}

${ }^{1}$ Significant difference at $\alpha=0.05$.

treatments, except that survival was 45 percent $( \pm 29)$ in 1,400 $\mathrm{mg} / \mathrm{L} \mathrm{NaHCO}_{3}$ exposures.

A second experiment was performed to validate the repeatability of the hatching success of white suckers. After 22 days of the initial white sucker spawning, 10 female and 14 male white suckers from the Beaverhead River pond were spawned and the eggs transported to the Jackson Field Research Station. At least 250 eggs were placed in a 4-L glass aquaria and exposed to $\mathrm{NaHCO}_{3}$ using the methods described in Methods, White Sucker Experiment section above. Hatching success and survival of the second group of eggs spawned was similar to the first group (data not presented).

Although survival was unaffected (table 3-15) by the exposures to $\mathrm{NaHCO}_{3}$, mean lengths and weights of white suckers from all treatments were significantly less than those of fish in the control treatment (table 3-16). The responses followed a concentration-response pattern with the smallest mean lengths and weights measured in fish exposed to $1,400 \mathrm{mg}$ $\mathrm{NaHCO}_{3} / \mathrm{L}$. Based on weight, the IC20 for white suckers was $348 \mathrm{mg} \mathrm{NaHCO} / \mathrm{L}$ (95-percent CI, 327-445 mg NaHCO$/ \mathrm{L}$ ).

The histological examination revealed bacterial kidney disease in control fish, but not in fish exposed to varying concentrations of $\mathrm{NaHCO}_{3}$. In general the severity of gill, kidney, gastrointestinal tract, and liver lesions was related directly to $\mathrm{NaHCO}_{3}$ exposure concentration and the duration of exposure. Laboratory rearing of wild fish and specific culture conditions (for example, feed) may account for some of the variability among groups. However, liver lesions clearly progressed from cytoplasmic degeneration and cystic change to nuclear degeneration and preneoplastic change as exposure concentration and duration of exposure increased. Statistical evaluations were not performed on whole-body ion concentrations (tables 3-17, 3-18). 
Table 3-17. Mean whole-body ions in white suckers (Catostomus commersoni) on day 30 of a chronic experiment completed in reconstituted Tongue River water (control). Exposure concentrations presented are nominal values of sodium bicarbonate. Sample size equals three.

$\left[\mathrm{NaHCO}_{3}\right.$, sodium bicarbonate; $\mathrm{mg} / \mathrm{L}$, milligrams per liter; $\mathrm{mg} / \mathrm{g}$, milligrams per gram; ( ), standard deviation $]$

\begin{tabular}{|c|c|c|c|c|}
\hline $\begin{array}{c}\text { Exposure } \\
\left(\mathrm{mg} \mathrm{NaHCO}_{3} / \mathrm{L}\right)\end{array}$ & $\begin{array}{c}\text { Calcium } \\
(\mathrm{mg} / \mathrm{g})\end{array}$ & $\begin{array}{l}\text { Magnesium } \\
(\mathrm{mg} / \mathrm{g})\end{array}$ & $\begin{array}{c}\text { Potassium } \\
\text { (mg/g) }\end{array}$ & $\begin{array}{c}\text { Sodium } \\
(\mathrm{mg} / \mathrm{g})\end{array}$ \\
\hline \multirow[t]{2}{*}{ Control } & 15.62 & 1.67 & 20.49 & 10.85 \\
\hline & $(9.75)$ & (.43) & (3.45) & (2.4) \\
\hline \multirow[t]{2}{*}{450} & 12.91 & 1.59 & 18.93 & 10.47 \\
\hline & $(3.24)$ & (.44) & (4.78) & (2.11) \\
\hline \multirow[t]{2}{*}{800} & 16.27 & 1.63 & 19.3 & 10.52 \\
\hline & (2.99) & (.9) & (1.61) & (1.3) \\
\hline \multirow[t]{2}{*}{1,400} & 20.2 & 1.8 & 20.3 & 11.12 \\
\hline & (9.12) & $(.41)$ & $(3.32)$ & (1.63) \\
\hline
\end{tabular}

Table 3-18. Mean whole-body ions in white suckers (Catostomus commersoni) on day 53 of a chronic experiment completed in reconstituted Tongue River water (control). Exposure concentrations presented are nominal values of sodium bicarbonate. Sample size equals two for all exposures except $450 \mathrm{mg} \mathrm{NaHO} / \mathrm{L}$ where sample size equals three. Statistics were not performed on these data.

$\left[\mathrm{NaHCO}_{3}\right.$, sodium bicarbonate; $\mathrm{mg} / \mathrm{L}$, milligrams per liter; $\mathrm{mg} / \mathrm{g}$, milligrams per gram; ( ), standard deviation]

\begin{tabular}{ccccc}
\hline $\begin{array}{c}\text { Exposure } \\
(\mathbf{m g ~ N a H C O} / \mathbf{L})\end{array}$ & $\begin{array}{c}\text { Calcium } \\
(\mathbf{m g} / \mathbf{g})\end{array}$ & $\begin{array}{c}\text { Magnesium } \\
(\mathbf{m g} / \mathbf{g})\end{array}$ & $\begin{array}{c}\text { Potassium } \\
(\mathbf{m g} / \mathbf{g})\end{array}$ & $\begin{array}{c}\text { Sodium } \\
(\mathbf{m g} / \mathbf{g})\end{array}$ \\
\hline Control & 7.62 & 1.33 & 17.53 & 8.44 \\
& $(0.39)$ & $(.01)$ & $(1.17)$ & $(.29)$ \\
450 & 9.36 & 1.75 & 22.46 & 9.68 \\
& $(2.12)$ & $(0.51)$ & $(6.71)$ & $(3.51)$ \\
800 & 12.02 & 1.63 & 19.52 & 9.31 \\
& $(1.76)$ & $(.16)$ & $(2.09)$ & $(1.77)$ \\
\hline
\end{tabular}

\section{Summary Results White Suckers}

The estimated percent hatch for white suckers was less than 30 percent in all treatments. This percent hatch was less than that observed for FHM, but it is not unexpected because the white sucker eggs were obtained from wild fish and were incubated in the laboratory. Effects on the survival of white suckers exposed to $\leq 1,400 \mathrm{mg} \mathrm{NaHCO} / / \mathrm{L}$ were not observed. However, lengths and weights were reduced in exposures $>450 \mathrm{mg} \mathrm{NaHCO} / \mathrm{L}$. Though whole-body ion concentrations do not explain the mechanism of toxicity, the presence of bacterial kidney disease in the controls may have masked the usefulness of the whole-body ion measurements. Also, there is a question of whether or not the presence of some concentrations of $\mathrm{NaHCO}_{3}$ minimized the detection of bacterial kidney disease in fish or alleviated the disease expression. Histological examinations point to progressive liver lesions as the concentrations and duration of exposures continued and these lesions may have been linked to the observations of decreased lengths and weights.

\section{Ceriodaphnia Experiment}

The survival and reproduction of C. dubia in control water was 100 percent and 26 neonates/adult, respectively, which meet the requirements for a successful experiment (table 3-19). The estimated 7-d LC50 was $1,192 \mathrm{mg}$ $\mathrm{NaHCO}_{3} / \mathrm{L}$ with a 95 percent $\mathrm{CI}$ of $963-1,476 \mathrm{mg} \mathrm{NaHCO} / \mathrm{L}$. The number of neonates produced was the largest in the moderately hard reconstituted water control and smallest in the 1,500 $\mathrm{mg} \mathrm{NaHCO}_{3} / \mathrm{L}$ exposure (table 3-19). The 7-d IC20 based on reproduction was $359 \mathrm{mg} \mathrm{NaHCO} / \mathrm{L}$ (95-percent $\mathrm{CI}=258-506$ ). This can also be reported as $274 \mathrm{mg} \mathrm{HCO}_{3}^{-/} / \mathrm{L}$ (95-percent $\mathrm{CI}=190-377$ ) and total alkalinity of 249 expressed as $\mathrm{mg} \mathrm{CaCO}_{3} / \mathrm{L}$ (95-percent $\left.\mathrm{CI}=173-354\right)$. 


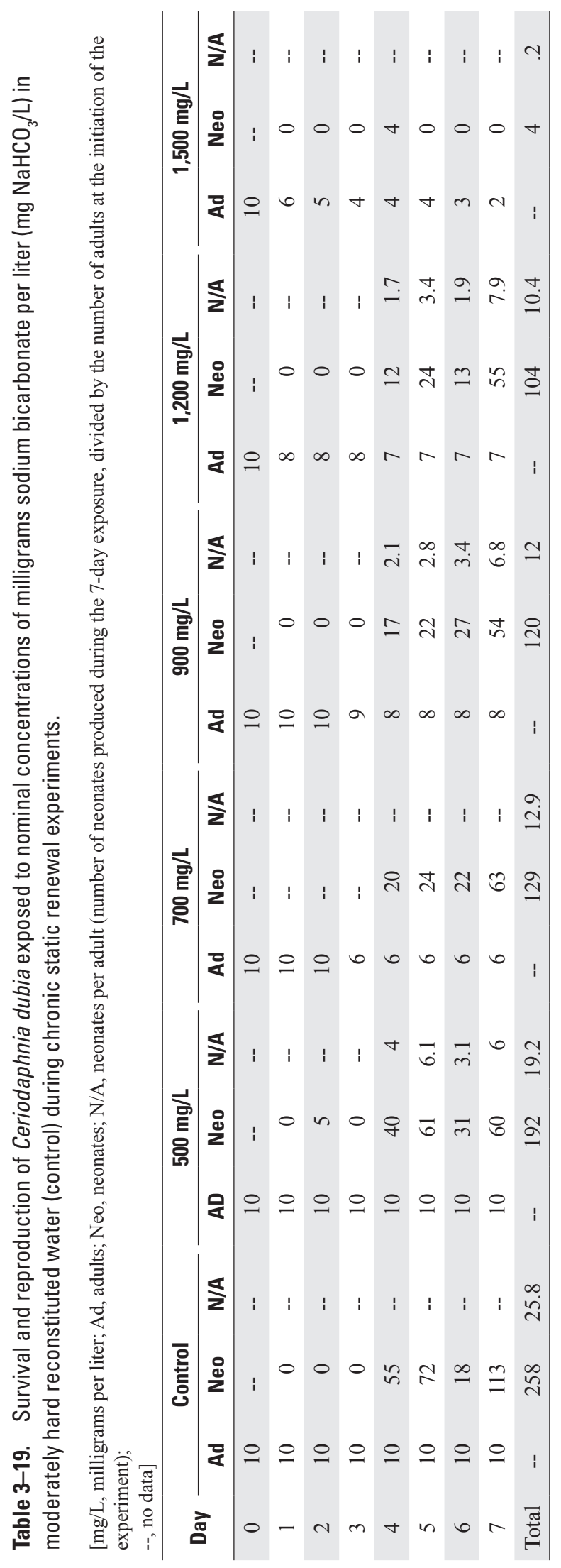


Table 3-20. Survival of 10-day old newly transformed fatmucket mussels (Lampsilis siliquoidea) to chronic exposures completed in reconstituted Tongue River water (control). Exposure concentrations presented are nominal values of sodium bicarbonate.

$\left[\mathrm{NaHCO}_{3}\right.$, sodium bicarbonate; $\mathrm{mg} / \mathrm{L}$, milligrams per liter; ( ), number of alive individuals per exposure, in percent]

\begin{tabular}{ccccc}
\hline $\begin{array}{c}\text { Exposure } \\
\left(\mathbf{m g ~ N a H C O}_{\mathbf{3}} / \mathbf{L}\right)\end{array}$ & $\mathbf{0 ~ d a y s}$ & $\mathbf{4}$ days & $\mathbf{6}$ days & $\mathbf{1 0 \text { days }}$ \\
\hline Control & $20(100)$ & $20(100)$ & $20(100)$ & $20(100)$ \\
500 & $20(100)$ & $20(100)$ & $20(100)$ & $20(100)$ \\
750 & $20(100)$ & $19(95)$ & $19(95)$ & $19(95)$ \\
1,000 & $20(100)$ & $16(80)$ & $13(65)$ & $13(65)$ \\
1,250 & $20(100)$ & $9(45)$ & $6(30)$ & $4(20)$ \\
1,500 & $20(100)$ & $2(10)$ & $0(0)$ & $0(0)$ \\
2,000 & $20(100)$ & $0(0)$ & $0(0)$ & $0(0)$ \\
\hline
\end{tabular}

\section{Freshwater Mussel Experiment}

The lack of foot movement during a 5-minute observation period was the effect endpoint monitored during the mussel experiment. Also, mussels that did not show foot movement were considered to be dead. Mussels exposed to $1,000 \mathrm{mg}$ $\mathrm{NaHCO}_{3} / \mathrm{L}$ for 4 days had 16 percent survival and 2 percent of mussels exposed to $1,500 \mathrm{mg} \mathrm{NaHCO} / / \mathrm{L}$ survived. Additional mortalities were observed in mussels exposed to 1,000 and $1,250 \mathrm{mg} \mathrm{NaHCO}_{3} / \mathrm{L}$, but survival in all other experimental concentrations remained constant until day 10 (table 3-20).

The 10-d EC50 for newly transformed juvenile fatmuckets was $1,061 \mathrm{mg} \mathrm{NaHCO} / \mathrm{L}$ (95-percent CI, 990-1,137 mg $\mathrm{NaHCO}_{3} / \mathrm{L}$ ). The 10-d IC20 based on foot movement was calculated to be $952 \mathrm{mg} \mathrm{NaHCO} / \mathrm{L}$ (95-percent CI, $828-1,076 \mathrm{mg} \mathrm{NaHCO}_{3} / \mathrm{L}$ ). This also can be expressed as $715 \mathrm{mg} \mathrm{HCO}_{3}^{-/} / \mathrm{L}$ (95-percent CI, 626-805 $\mathrm{mg} \mathrm{HCO}_{3}^{-/} / \mathrm{L}$ ) and

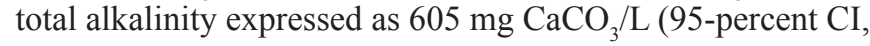
508-680 expressed as $\mathrm{mg} \mathrm{CaCO}_{3} / \mathrm{L}$ )

\section{Discussion}

Regardless of the species (fish, crustacean, or bivalve) or type of effect measured (growth, survival, or reproduction), data from the present study demonstrated approximately 500 $1,000 \mathrm{mg} \mathrm{NaHCO} / \mathrm{L}$ affects aquatic animals in chronic exposure conditions. Also, this range of $\mathrm{NaHCO}_{3}$ concentrations elicited effects regardless of whether animals were cultured in controlled conditions in the laboratory (for example, C. dubia; FHM) or spawned in the wild (white sucker). Compared to the other species tested, freshwater mussels were the least sensitive to $\mathrm{NaHCO}_{3}$ exposure, especially in relation to the IC20 for C. dubia. The IC20 for C. dubia and freshwater mussels is 359 and $952 \mathrm{mg} \mathrm{NaHCO} / \mathrm{L}$, respectively.

When survival is the only consideration, the FHM appear more sensitive than white sucker and incurred significant reductions in survival in exposure concentrations $>500$ or $>1,100 \mathrm{mg} \mathrm{NaHCO}_{3} / \mathrm{L}$ depending on the type of experiment, whereas no effects on survival of white sucker were observed in any of the exposure concentrations. However, growth effects were observed in white sucker exposed to concentrations as low as $450 \mathrm{mg} \mathrm{NaHCO} / \mathrm{L}_{3}$ compared to $650 \mathrm{mg}$ $\mathrm{NaHCO}_{3} / \mathrm{L}$ for FHM. And the growth effects documented in white sucker were substantiated by histological findings that revealed progressive liver disease with increased exposure concentrations. If both survival and growth effects are considered, the results of the chronic fish experiments define effects at the lower end of the range of $\mathrm{NaHCO}_{3}$ concentrations investigated.

To investigate the mechanisms of toxicity of $\mathrm{NaHCO}_{3}$, $\mathrm{Na} / \mathrm{K}$ ATPase induction and whole-body ion concentrations were studied. These measurements along with the pattern of mortality and growth effects between the fish species revealed changes that may occur with exposure to $\mathrm{NaHCO}_{3}$. Because $\mathrm{Na} / \mathrm{K}$ ATPase is involved, at least in part, in the regulation of $\mathrm{Na}^{+}$in freshwater fish (Marshall, 2002) and the concomitant movement of $\mathrm{HCO}_{3}^{-}$once an ion gradient is established, the expectation was that concentrations of $\mathrm{Na} / \mathrm{K}$ ATPase would be elevated during $\mathrm{NaHCO}_{3}$ exposures. This expectation was based on the hypothesis that an induction of the enzyme system would assist the animal in regulating ion status. Quite to the contrary, the concentrations of $\mathrm{Na} / \mathrm{K}$ ATPase were reduced in fathead minnow exposed to elevated $\mathrm{NaHCO}_{3}$. Other researchers have documented decreases in ATPase as a result of $\mathrm{NaHCO}_{3}$ exposure. For example, a decrease in N-ethylmaleimide (NEM)-sensitive ATPase of the ascending limb and outer medullar collecting tubule of the nephron was observed in rats as a result of metabolic alkalosis induced by a $\mathrm{NaHCO}_{3}$ exposure (Khadouri and others, 1992).

In the present study, FHM exposed to 300 or $400 \mathrm{mg}$ $\mathrm{NaHCO}_{3} / \mathrm{L}$ did not exhibit any effects on growth or survival; however, $\mathrm{Na} / \mathrm{K}$ ATPase was reduced significantly at day 60 , but not day 37 in fish exposed to these concentrations compared to the controls. The reduced $\mathrm{Na} / \mathrm{K}$ ATPase concentration in FHM exposed to the 300 or $400 \mathrm{mg} \mathrm{NaHCO} / \mathrm{L}$ was reduced to a lesser extent than in fish exposed to concentrations $>400$ $\mathrm{mg} \mathrm{NaHCO}_{3} / \mathrm{L}$ where effects on survival were observed. In 
addition, fish had an early onset of significantly decreased $\mathrm{Na} / \mathrm{K}$ ATPase at day 37 in all but the smallest $(500 \mathrm{mg} / \mathrm{L}$ $\mathrm{NaHCO}_{3} / \mathrm{L}$ ) concentration that resulted in significant mortality. The significant decrease in $\mathrm{Na} / \mathrm{K}$ ATPase together with the lack of growth effects suggests that the $\mathrm{Na} / \mathrm{K}$ ATPase activity was actually shut down before the onset of death. The decrease rather than increase in $\mathrm{Na} / \mathrm{K}$ ATPase may explain why effects on the growth of FHM were not observed. Energy for growth was not diverted to enzyme induction. Although FHM were tested under $\mathrm{NaHCO}_{3}$ chronic exposure conditions, the resulting ionic imbalance may, in fact be an acute response mediated through an extreme ionic gradient that overwhelmed the animal's ability to maintain ion regulation. As a result, $\mathrm{Na} / \mathrm{K}$ ATPase no longer functioned in the animal and death occurred.

When the magnitude of $\mathrm{Na} / \mathrm{K}$ ATPase decreases in FHM exposed to $\mathrm{NaHCO}_{3}$ are compared, it appears percent decrease in the activity of $\mathrm{Na} / \mathrm{K}$ ATPase and the age of the fish at the onset of the decrease may affect the ability of FHM to survive. For example, fish were able to survive an 8.12 percent decrease in $\mathrm{Na} / \mathrm{K}$ ATPase activity in the $400 \mathrm{mg} \mathrm{NaHCO} / \mathrm{L}_{2}$ treatment compared to the control when this decrease was first documented on day 60 . However, fish with an 8.50 percent decrease in $\mathrm{Na} / \mathrm{K}$ ATPase activity first documented on day 37 in the $500 \mathrm{mg} \mathrm{NaHCO} / \mathrm{L}$ treatment incurred significant reductions in survival and subsequently exhibited a 26.5 percent reduction in $\mathrm{Na} / \mathrm{K}$ ATPase at day 60 . The effects of $\mathrm{NaHCO}_{3}$ on $\mathrm{Na} / \mathrm{K}$ ATPase activity and the ability of fish to survive likely are related to the age of the fish at exposure. $\mathrm{Na} / \mathrm{K}$ ATPase was not measured in fish from the 7-d FHM experiment where growth effects were observed. Such measurements could shed more light on the potential effect of $\mathrm{Na} / \mathrm{K}$ ATPase on $\mathrm{NaHCO}_{3}$ toxicity.

The changes in whole-body ion concentrations appear to be related indirectly to $\mathrm{NaHCO}_{3}$ exposure. Significant elevations of whole-body $\mathrm{Ca}$ and $\mathrm{Mg}$ occurred in FHM exposed to the higher range of the $\mathrm{NaHCO}_{3} / \mathrm{L}$ treatments that appeared to be associated with the reduced amount of $\mathrm{Ca}$ in solution. These changes were noteworthy in FHM and white suckers, but the data could not be statistically tested because of small sample sizes. A white precipitate was observed on the experimental equipment in the greater $\mathrm{NaHCO}_{3}$ concentrations. It is possible that some of the whole-body Ca measured was actually precipitates that settled onto the fish. The one instance of increased whole-body $\mathrm{Na}$ in FHM was observed in the greatest concentration of $1,100 \mathrm{mg} \mathrm{NaHCO} / / \mathrm{L}$ on day 37 . Thus, wholebody ion concentrations do not appear to be an adequately sensitive indicator of $\mathrm{NaHCO}_{3}$ toxicity. Whole-body ion measurements do not detect changes among extracellular and intracellular ion concentrations, where the ionoregulation effects in this situation are likely to occur.

Several histological anomalies suggested that fish were adversely affected as a result of exposure to $>450 \mathrm{mg}$ $\mathrm{NaHCO}_{3} / \mathrm{L}$. For example, necrotic (dying) cells were found in gills of FHM and the incidence of necrotic cells increased with exposure concentration. The presence of necrotic cells may have precluded the observation of a proliferation of mitochondrial rich cells. Changes in kidney, intestine, and livers were observed in white sucker as well as progressive liver disease in relation to exposure concentration, which may explain the observed adverse effects on the white sucker.

The histological investigation also detected the presence of bacterial kidney disease in white suckers held in the control water during the experiment. This disease may, at least in part, explain the low hatch success of white sucker embryos. The lack of bacterial kidney disease in the exposed white sucker also may be related to the greater percent hatch of white sucker embryos exposed to some of the $\mathrm{NaHCO}_{3}$ treatments. A white film that appeared to be fungus accumulated around the white sucker eggs early in the experiment. The amounts of the film that accumulated on eggs, but could not be quantified, were related indirectly to the $\mathrm{NaHCO}_{3}$ exposure concentrations; however, the white suckers continued to hatch in the presence of this film. Likely the presence of the $\mathrm{NaHCO}_{3}$ salt protected the eggs during incubation. Salt has been used by hatchery personnel to treat fish during transport to minimize stress and disease expression (Piper and others, 1982). However, when the white sucker embryos hatched, the $\mathrm{NaHCO}_{3}$ affected their ability to grow to the same extent as the fish in the control treatment, and was associated with progressive liver disease. All diluter equipment was completely disinfected according to USGS protocol and no white suckers from the laboratory were released into the wild.

The natural background concentration of salts in the Tongue and Powder Rivers made it necessary to use control water for chronic toxicity experiments that mimicked the natural conditions. Simulated Tongue River water was used for all the chronic toxicity experiments, with the exception of the $C$. dubia. The C. dubia experiment was completed with moderately hard reconstituted water in accordance with U.S. Environmental Protection Agency (1994) suggested guidelines. In theory, the use of the simulated Tongue River water could have defined lesser effects compared to this control water because animals were exposed to some salt concentrations in the control. For this reason, one might consider the use of the simulated Tongue River as a conservative approach. However, the C. dubia experiment used a moderately hard reconstituted water and the IC20 effects on reproduction was defined at $359 \mathrm{mg} \mathrm{NaHCO} / / \mathrm{L}$, similar to effects at 450 and $500 \mathrm{mg}$ $\mathrm{NaHCO}_{3} / \mathrm{L}$ noted for FHM and white suckers, respectively. Additional work may be initiated with C. dubia in simulated Tongue River water to further define any specific differences between control waters with this species.

The use of the simulated Tongue River water as a control does not, however, preclude the use of these data in other watersheds where $\mathrm{NaHCO}_{3}$ may be introduced to a system. The repeatability of effects defined on multiple species of both invertebrates and fish was noted at concentrations above 500-1,000 $\mathrm{mg} \mathrm{NaHCO}_{3} / \mathrm{L}$. The effects were repeatable and included sublethal (for example, growth and reproduction) and lethal (survival) endpoints; and the pattern of Na/K ATPase reduction suggests that the system shuts down before the 
onset of death. These data can be used for comparisons by other regions regardless of the reference water present in that particular region.

The overall results of these experiments suggest that there is minimal room for $\mathrm{NaHCO}_{3}$ increases in the Tongue River/ Powder River watershed (fig. 1-2) if a goal is to maintain the health of aquatic life. In the end, it was difficult to bracket effects with no effect concentrations because decreased survival (fathead minnow) at $450 \mathrm{mg} \mathrm{NaHCO}_{3} / \mathrm{L}$ and decreased growth (white sucker) at $500 \mathrm{mg} \mathrm{NaHCO} / \mathrm{L}$ was observed. Concentrations during the low-flow period (late summer and early fall) are generally 250 and $350 \mathrm{mg} \mathrm{NaHCO} / /$ in the main stem of the Tongue and Powder Rivers, respectively. To date, concentrations of $\mathrm{NaHCO}_{3}$ determined to adversely affect biota in this study have been observed in some tributaries, but have not been observed in the main stem of the Tongue and Powder Rivers (Peterson and others, 2010; chapter 4, Discussion section). Continued monitoring of water quality throughout the Tongue River/Powder River watershed is needed to identify exceedances of the $\mathrm{NaHCO}_{3}$ effect concentrations determined in this study.

\section{References Cited}

American Public Health Association Standard Methods, 1975, Alkalinity titration method for water and wastewater (14th ed.): Washington, D.C., American Public Health Association, p. 278.

American Society of Testing and Materials International, 2006, Standard guide for conducting laboratory toxicity tests with freshwater mussels: West Conshohocken, Pa., American Society of Testing and Materials International, E 2455-06, $52 \mathrm{p}$.

ALL Consulting, 2003, Handbook on coalbed methane produced water-Management and beneficial use alternatives: coalbed natural gas resources and produced water management, Tulsa, ALL Consulting, Report to the U.S. Department of Energy, accessed April 2010 at $h t t p: / / w w w$.all-llc. com/CBNG/BU/index.htm, $44 \mathrm{p}$.

Farag, A.M., Skaar, D., Nimick, D.A., MacConnell, E., and Hogstrand, C., 2003, Characterizing aquatic health using salmonid mortality, physiology, and biomass estimates in streams with elevated concentrations of arsenic, cadmium, copper, lead, and zinc in the Boulder River Watershed, Montana: Transactions of the American Fisheries Society, v. 132 , p. $450-467$.

Khadouri, C., Marsy, S., Barlet-bas, C., Cheval, L., and Doucet, A., 1992, Effect of metabolic acidosis and alkalosis on NEM-sensitive ATPase in rat nephron segments: American Journal of Physiology, v. 262, p., F583-F590.
Marshall, W.S., 2002, $\mathrm{Na}^{+}, \mathrm{Cl}^{-}, \mathrm{Ca}^{+}$, and $\mathrm{Zn} 2^{+}$Transport by fish gills-Retrospective review and prospective synthesis: Journal of Experimental Zoology, v. 293, p. 264-283.

Mount, D.R., Gulley, D.D., Hockett, J.R., Garrison, T.D., and Evans, J.M., 1997, Statistical models to predict the toxicity of major ions to Ceriodaphnia dubia, Daphnia magna, and Pimephales promelas (fathead minnows): Environmental Toxicology and Chemistry, v. 16, p. 2,009-2,019.

Mount, D.I., and Brungs, W.A., 1967, A simplified dosing apparatus for fish toxicology studies: Water Research, v. 1, p. 21-29.

Norberg-King, T.J., 1993. A linear interpolation method for sublethal toxicity-The inhibition concentration (ICp) approach (Version 2.0.): Duluth, U.S. Environmental Protection Agency, National Effluent Toxicity Assessment Center Technical Report 03-93, 39 p.

Patz, M.J., Reddy, K.J., and Skinner, Q.D., 2004, Chemistry of coalbed methane discharge water interacting with semi-arid ephemeral stream channels: Journal of the American Water Resources Association, v. 40, no. 5, p. 1,247-1,255.

Peterson, D.A., Clark, M.A., Foster, K., Wright, D.L., and Boughton, J.K., 2010, Assessment of ecological conditions and potential effects of water produced from coalbed natural gas development on biological communities in streams of the Powder River structural basin, Wyoming and Montana, 2005-08: U.S. Geological Survey Scientific Investigations Report 2010-5124, 84 p.

Piper, R.G., McElwain, I.B., Orme, L.E., McCraren, J.P., Fowler, L.G., and Leonard, J.R., 1982, Fish hatchery management: Washington, D.C., U.S. Fish and Wildlife Service, $317 \mathrm{p}$.

Reid, S.D., and McDonald, D.G., 1988, Effects of cadmium, copper, and low pH on ion fluxes in rainbow trout, Salmo gairdneri: Canadian Journal of Fisheries and Aquatic Sciences, v. 45, p. 244-253.

Stephan, C.E., Mount, D.I., Hansen, D.J., Gentile, J.R., Chapman, G.A., and Brungs, W.A., 1985, Guidelines for deriving numerical national water quality criteria for the protection of aquatic organisms and their uses: Duluth, U.S. Environmental Protection Agency, Office of Research and Development, EPA-PB85-227049, 106 p.

Wang, N., Augspurger, T., Barnhart, M.C., Bidwell, J.R., Cope, W.G., Dwyer, F.J., Geis, S., Greer, I.E., Ingersoll, C.G., Kane, C.M., May, T.W., Neves, R.J., Newton, T. J., Roberts, A.D., and Whites, D.W., 2007, Intra- and Interlaboratory variability in acute toxicity tests with glochidia and juveniles of freshwater mussels (unionidae): Environmental Toxicology and Chemistry, v. 26, p. 2,029-2,035. 
Wilson, J.M., and P. Laurent, 2002, Fish gill morphologyInside Out: Journal of Experimental Zoology, v. 293, p. 192-213.

U. S. Environmental Protection Agency, 1994, Short term methods for estimating the chronic toxicity of effluents and receiving waters to aquatic organisms, in P.A. Lewis, Klemm, D.J., Lazorchak, J.M., Norberg-King, T.J., Peltier, W.H., and Heber, M.A. eds., Duluth, U.S. Environmental Protection Agency, EPA/600/4 -91/002, 315 p.

U.S. Environmental Protection Agency, 2010, The Toxicity Relationship Analysis Program: Duluth, U.S. Environmental Protection Agency, Mid-Continent Ecology Division, accessed April 25, 2011, at http://www.epa.gov/med/Prods Pubs/trap.htm. 


\section{The Potential Toxicity Defined In Situ and Streamside in the Powder and Tongue River Watersheds}

By Aïda M. Farag, David D. Harper, and Don Skaar

Chapter 4 of

The Potential Effects of Sodium Bicarbonate, a Major Constituent of Produced Waters from Coalbed Natural Gas Production, on Aquatic Life

Edited by Aïda M. Farag and David D. Harper

Prepared in cooperation with Montana Fish, Wildlife, and Parks, U.S. Bureau of Land Management, and the U.S. Environmental Protection Agency

Scientific Investigations Report 2012-5008 


\section{Contents}

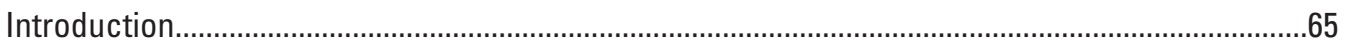

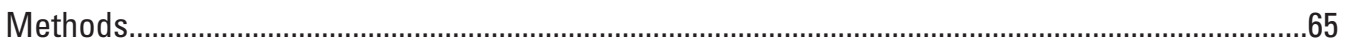

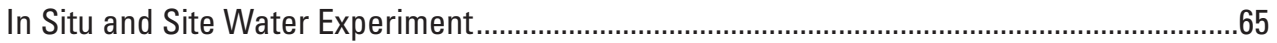

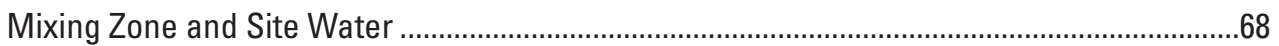

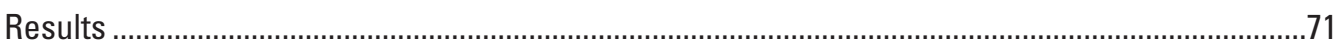

Site Water/Mixing Zone Experiments...................................................................................

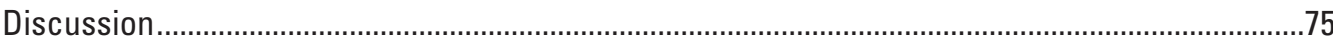

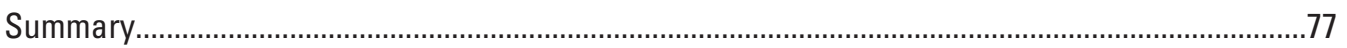

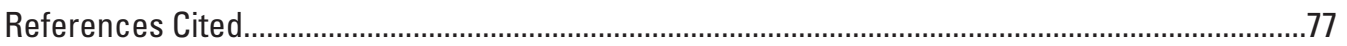

\section{Figures}

4-1. Map showing field sites for 96-hour in situ toxicity experiments in the Powder River Drainage, Wyoming

4-2. Graph showing percent survival at 96 hours for in situ experiments started with 2-daypost-hatch (2-dph) or 6-day-post-hatch (6-dph) fathead minnows. Powder River drainage, July-August 2006

4-3. Graph showing percent survival at 96 hours for the mixing zone experiment started with 2-day-post-hatch fathead minnows. Receiving water was added to experimental waters to provide the percent mixture indicated. Sample waters were collected from the Powder and Tongue Rivers watershed, September-October 2007

\section{Tables}

4-1. Mean alkalinity expressed as milligrams per liter calcium carbonate equivalent, bicarbonate, calcium, magnesium, sodium, potassium, chloride, sulfate, and ammonia ( $\mathrm{mg} \mathrm{N} / \mathrm{L}$ ) during in situ experiments with 2- and 6-day-post-hatch fathead minnows (Pimephales promelas) performed in the Powder River Basin in Wyoming and Montana.....

4-2. Mean water chemistry recorded with hand-held meters during in situ toxicity experiments with fathead minnows (Pimephales promelas) in the Powder River Basin in Wyoming and Montana

4-3. Minimum and maximum temperature, conductivity, dissolved oxygen, and $\mathrm{pH}$ measurements recorded every 30 minutes at four sites during in situ experiments with fathead minnows (Pimephales promelas) in the Powder River Basin, Wyoming and Montana. Data was recorded with YSI $5000^{\text {TM }}$ Data Sonde units

4-4. Additional trace metal scans analyzed with Inductively Coupled Plasma (ICP) in water samples collected from in situ experimental sites for fathead minnows (Pimephales promelas). Experiments completed in the Powder River Basin, Wyoming and Montana. Sample size is 1 for all constituents

4-5. Mean alkalinity expressed as milligrams per liter calcium carbonate equivalent, bicarbonate, sulfate, calcium, magnesium, sodium, and potassium, during in situ experiments with 4- and 6-day-post-hatch pallid sturgeon (Scaphirhynchus albus) performed in the Powder River Basin in Wyoming.

4-6. Mean temperature, conductivity, dissolved oxygen, and pH measurements recorded with hand-held meters during in situ toxicity experiments with pallid sturgeon (Scaphirhynchus albus) in the Powder River Basin in Wyoming. 
4-7. Additional trace metal scans analyzed with Inductively Coupled Plasma (ICP) in water samples collected from in situ experimental sites with pallid sturgeon (Scaphirhynchus albus) completed in the Powder River Basin, Wyoming....

4-8. Mean concentrations of ammonia, in water from experimental sites during in situ experiments performed in the Powder River Basin, Wyoming. .71

4-9. Mean alkalinity expressed as milligrams per liter calcium carbonate equivalent, bicarbonate, sulfate, calcium, magnesium, sodium, potassium, and total ammonia (mg $\mathrm{N} / \mathrm{L}$ ) during 96-hour static renewal exposures of 2-day-post-hatch fathead minnows (Pimephales promelas) to mixtures of waters collected in the Tongue and Powder River Basins, Wyoming and Montana, September 2007.

4-10. Survival of fathead minnows (Pimephales promelas) during 96-hour in situ experiments in the Powder River Basin, Wyoming and Montana. Two experiments were performed with fathead minnows of different ages. Fish were 2-day-post-hatch (dph) at the initiation of experiment 1 and were 6 -day-post-hatch at the initiation of experiment 2 .........73

4-11. Survival of pallid sturgeon (Scaphirhynchus albus) during 96-hour in situ experiments in the Powder River Basin, Wyoming. Fish were 4 or 6-day-post-hatch (dph) at the initiation of the experiments. . .73

4-12. Dye concentrations as measured in transects across the Powder River downstream from the confluence of Beaver Creek. Beaver Creek entered on the right bank and distances at each transect were measured in meters from the right stream bank to the left stream bank . .74

4-13. Dye concentrations as measured in transects across the Tongue River downstream from diffusers injecting untreated coal bed natural gas product water. Diffusers were located on the right bank and distances at each transect were measured in meters from the right stream bank to the left stream bank..

4-14. Dye concentrations as measured in transects across the Tongue River downstream from diffusers injecting treated coal bed natural gas product water. Diffusers were located on the right bank and distances at each transect were measured in meters from the right stream bank to the left stream bank. 



\title{
The Potential Toxicity Defined In Situ and Streamside in the Powder and Tongue River Watersheds
}

\author{
By Aïda M. Farag', David D. Harper'1, and Don Skaar²
}

\section{Introduction}

In situ toxicity experiments provide needed information about the ability of fish to survive in the field under real-world conditions (Farag and others, 2003; Harper and others, 2008). Reduced survival of fish may indicate the inability of a watershed to support an optimal population size (Farag and others, 2003). Therefore, these measurements can indicate impairment at the population level. The placement of sites for these studies also can provide indications about the source(s) of impairment. However, the overall goal when managing aquatic life is to avert or minimize conditions where the survivability of fish is affected. To achieve this management objective, laboratory experiments performed in controlled conditions are used to define threshold concentrations where effects of an element or compound may be observed on aquatic life. These threshold concentrations can then be used to alert managers and site operators about the potential effects of effluents that may be introduced into a water body.

As part of the effort to define threshold concentrations, in situ experiments can provide supporting data for laboratory derived thresholds. In situ experiments are especially critical when performed at sites where concentrations exceed laboratory derived thresholds, because the ability of fish to survive in real-world conditions can be determined. In this sense, field data are used to ground-truth laboratory derived concentrations before extensive catastrophic events where populations are affected. In situ experiments provide a link between the laboratory and the field to further assess potential effects in the field. The Powder/Tongue River watersheds provide a unique opportunity for field assessments because some tributaries in these watersheds were historically ephemeral, but now contain water year round as a result of discharge inputs of coalbed natural gas produced waters (Patz and others, 2004). As such, field sites with 100 percent effluent water could be studied in these watersheds.

Streamside experiments performed with site water are another alternative to gather field information. During these experiments, site water is used, but because the experiment is

${ }^{1}$ USGS, Columbia Environmental Research Center, Jackson Field Research Station, Jackson, Wyoming.

${ }^{2}$ Montana Fish, Wildlife, and Parks, Helena, Montana. initiated outside of the stream, some physical characteristics such as temperature and dissolved oxygen may be controlled to reduce potentially confounding factors. Stewart (1996) used these types of assays when attempting to assess ambient water conditions. Again, these types of experiments add useful information to data gathered from laboratory and in situ experiments because they add further evidence to the support or refute laboratory or in situ results.

Another opportunity for field studies in the Powder/ Tongue River watersheds is provided by mixing zones where tributaries, treated effluent, or untreated effluent flow into the main stem Tongue or Powder River. Research related to toxicity in mixing zones is limited. However, it has been documented that toxicity and related gill lesions were elevated in mixing zones that received acid-mine drainage (Henry and others, 2001). In this case, toxicity was related to metals that precipitated from solution in the mixing zone. In November 2006, the Aquatic Task Group (ATG) defined an investigation of the survivability of fish in mixing zones of the Powder and Tongue Rivers as the top priority for research needs still to be accomplished.

The main goal of this study was to investigate the survivability of fish in the field, at sites where concentrations of $\mathrm{NaHCO}_{3}$ exceeded threshold concentrations defined in the laboratory. A second goal of this study was to investigate the physical extent of the mixing zones at confluence points in the Tongue/Powder River watersheds.

\section{Methods}

\section{In Situ and Site Water Experiments}

Experiments to investigate the acute toxicity of produced waters in the Powder River Basin, Wyoming and Montana, were completed during July-August 2006 and July 2007. Early lifestage fathead minnow (FHM) Pimephales promelas and pallid sturgeon Scaphirhynchus albus (endemic species used in the laboratory experiments) were studied during 2006 and 2007, respectively. An additional static renewal experiment with pallid sturgeon was completed in controlled conditions with site-collected water in 2007. 
Experiments lasted in situ for 96-hours (h). Fish were held in 450 milliliters $(\mathrm{ml})$ cellulose acetate butyrate containers with 149 micrometers $(\mu \mathrm{m})$ mesh screen covering approximately 40 percent of the surface area. Each container held 10 fish and 4 replicate containers were deployed per site. In situ containers were held in place at each site by strapping the individual containers to a vinyl-coated metal dish drying rack that was placed in a perforated [118 liters (L)] Rubbermaid ${ }^{\text {тM }}$ HDPE storage tub. Storage tubs were held in place with stakes, rocks, and rope to prevent loss from potential flash flooding. At one site, the depth was not adequate to allow the use of the storage tub, and the dish drying rack was placed directly in the stream channel with a storage tub cover over the top to provide shade.

Experiments were initiated with 2-day post-hatch (dph) FHM on 26-29 July (experiment 1) and 6-dph FHM on 30 July-02 August, 2006 (experiment 2). Note: the terms "2-dph" and "6-dph" will be used from this point. Newly hatched fish were shipped directly to Buffalo, Wyoming from Aquatic Biosystems, Fort Collins, Colorado. The brood stock were deemed free of disease and kept in isolation at the Colorado facility. All relevant permits were obtained for importation of the offspring.

For experiment 1, two reference sites and four experimental sites were used; Powder River at Moorhead and Clear Creek (reference sites), SA Creek, Upper Beaver Creek, Lower Beaver Creek, and Burger Draw (experimental sites; fig. 4-1).
Following the completion of the 96-h experiments with 2-dph fish, experiment 2 was initiated with 6-dph FHM in situ at Clear Creek (reference site) Upper Beaver Creek, Lower Beaver Creek, and Burger Draw (experimental sites) with fish from the same source.

Survival was monitored every $24 \mathrm{~h}$, and water samples were collected daily from all sites. Water samples were placed on ice and filtered each evening with a $0.45 \mu \mathrm{m}$ Millipore $^{\mathrm{TM}}$ filter. Subsamples for cation analyses were acidified to less than $(<) \mathrm{pH} 2.0$ with ultrapure nitric acid and were held at 4 degrees Celsius $\left({ }^{\circ} \mathrm{C}\right)$ during storage and shipment for analyses. Anion samples were collected into bicarbonate rinsed containers and stored at $4^{\circ} \mathrm{C}$ during storage and shipment. Alkalinity (American Public Health Association Standard Methods, 1975a), hardness (American Public Health Association Standard Methods, 1975b), and $\mathrm{pH}$ were measured each evening (table 4-1). Water temperature, conductivity, and dissolved oxygen were measured manually at each site at least once daily (table 4-2), and monitored remotely with YSITM Data Sonde loggers at Powder River at Moorhead, Clear Creek, SA Creek and Lower Beaver Creek every 30 minutes (table 4-3). Ammonia was measured on-site daily with a $\mathrm{Hach}^{\mathrm{TM}}$ test kit (table 4-1). Additional trace metal scans were performed on one subsample of water from each site (table 4-4).

In 2007, 2-dph pallid sturgeon were transported from the Montana Fish Wildlife and Parks Miles City, Montana Fish Hatchery, to Buffalo, Wyoming. Pallid sturgeon at 2-, 4-, and 6-dph were exposed in experiments at; Clear Creek (reference

Table 4-1. Mean alkalinity expressed as milligrams per liter calcium carbonate equivalent, bicarbonate, calcium, magnesium, sodium, potassium, chloride, sulfate, and ammonia ( $\mathrm{mg} \mathrm{N} / \mathrm{L}$ ) during in situ experiments with 2- and 6-day-post-hatch fathead minnows (Pimephales promelas) performed in the Powder River Basin in Wyoming and Montana.

$\left[\mathrm{CaCO}_{3}\right.$, calcium carbonate; $\mathrm{mg} / \mathrm{L}$, milligrams per liter; mg N/L, milligrams of nitrogen per liter; ( ), standard deviation error; <, less than; N, sample size; --, no calculation of standard error was performed]

\begin{tabular}{|c|c|c|c|c|c|c|c|c|}
\hline Site & $\begin{array}{c}\text { Alkalinity } \\
\left(\mathrm{CaCO}_{3} \text {, in }\right. \\
\mathrm{mg} / \mathrm{L})\end{array}$ & $\begin{array}{l}\text { Bicarbonate } \\
\text { (mg/L) }\end{array}$ & $\begin{array}{l}\text { Sulfate } \\
\text { (mg/L) }\end{array}$ & $\begin{array}{c}\text { Calcium } \\
\text { (mg/L) }\end{array}$ & $\begin{array}{l}\text { Magnesium } \\
\text { (mg/L) }\end{array}$ & $\begin{array}{l}\text { Sodium } \\
\text { (mg/L) }\end{array}$ & $\begin{array}{l}\text { Potassium } \\
\text { (mg/L) }\end{array}$ & $\begin{array}{c}\text { Total } \\
\text { ammonia } \\
\text { (mg N/L) }\end{array}$ \\
\hline \multirow[t]{3}{*}{ Powder R. at Moorhead } & 202 & 196 & 1,767 & 197 & 185 & 660 & 32 & $<0.01$ \\
\hline & (11) & (10) & (36) & (49) & (43) & (162) & (11) & -- \\
\hline & $\mathrm{N}=5$ & $\mathrm{~N}=5$ & $\mathrm{~N}=3$ & $\mathrm{~N}=4$ & $\mathrm{~N}=4$ & $\mathrm{~N}=4$ & $\mathrm{~N}=3$ & $\mathrm{~N}=5$ \\
\hline Clear Creek & 198 & 192 & 285 & 74 & 52 & 68 & 5 & .05 \\
\hline \multirow[t]{3}{*}{ Lower Beaver } & 1,926 & 1,740 & 75 & 10 & 27 & 765 & 20 & .05 \\
\hline & (68) & $(128)$ & (1) & (.4) & $(.4)$ & (5) & $(0.2)$ & $(.03)$ \\
\hline & $\mathrm{N}=9$ & $\mathrm{~N}=9$ & $\mathrm{~N}=5$ & $\mathrm{~N}=6$ & $\mathrm{~N}=6$ & $\mathrm{~N}=6$ & $\mathrm{~N}=5$ & $\mathrm{~N}=5$ \\
\hline \multirow[t]{2}{*}{ Upper Beaver } & 1,759 & 1,646 & 257 & 16 & 24 & 664 & 20 & .5 \\
\hline & (66) & (64) & $(211)$ & (.4) & $(.2)$ & (6) & $(0.4)$ & $(.1)$ \\
\hline Burger Draw & $\mathrm{N}=9$ & $\mathrm{~N}=9$ & $\mathrm{~N}=5$ & $\mathrm{~N}=5$ & $\mathrm{~N}=5$ & $\mathrm{~N}=5$ & $\mathrm{~N}=5$ & $\mathrm{~N}=5$ \\
\hline \multirow[t]{3}{*}{ SA Creek } & 1,364 & 1,245 & 395 & 17 & 45 & 605 & 16 & .13 \\
\hline & (53) & (44) & (18) & (.6) & (.6) & (18) & (2) & $(.04)$ \\
\hline & $\mathrm{N}=5$ & $\mathrm{~N}=5$ & $\mathrm{~N}=3$ & $\mathrm{~N}=4$ & $\mathrm{~N}=4$ & $\mathrm{~N}=4$ & $\mathrm{~N}=3$ & $\mathrm{~N}=5$ \\
\hline
\end{tabular}




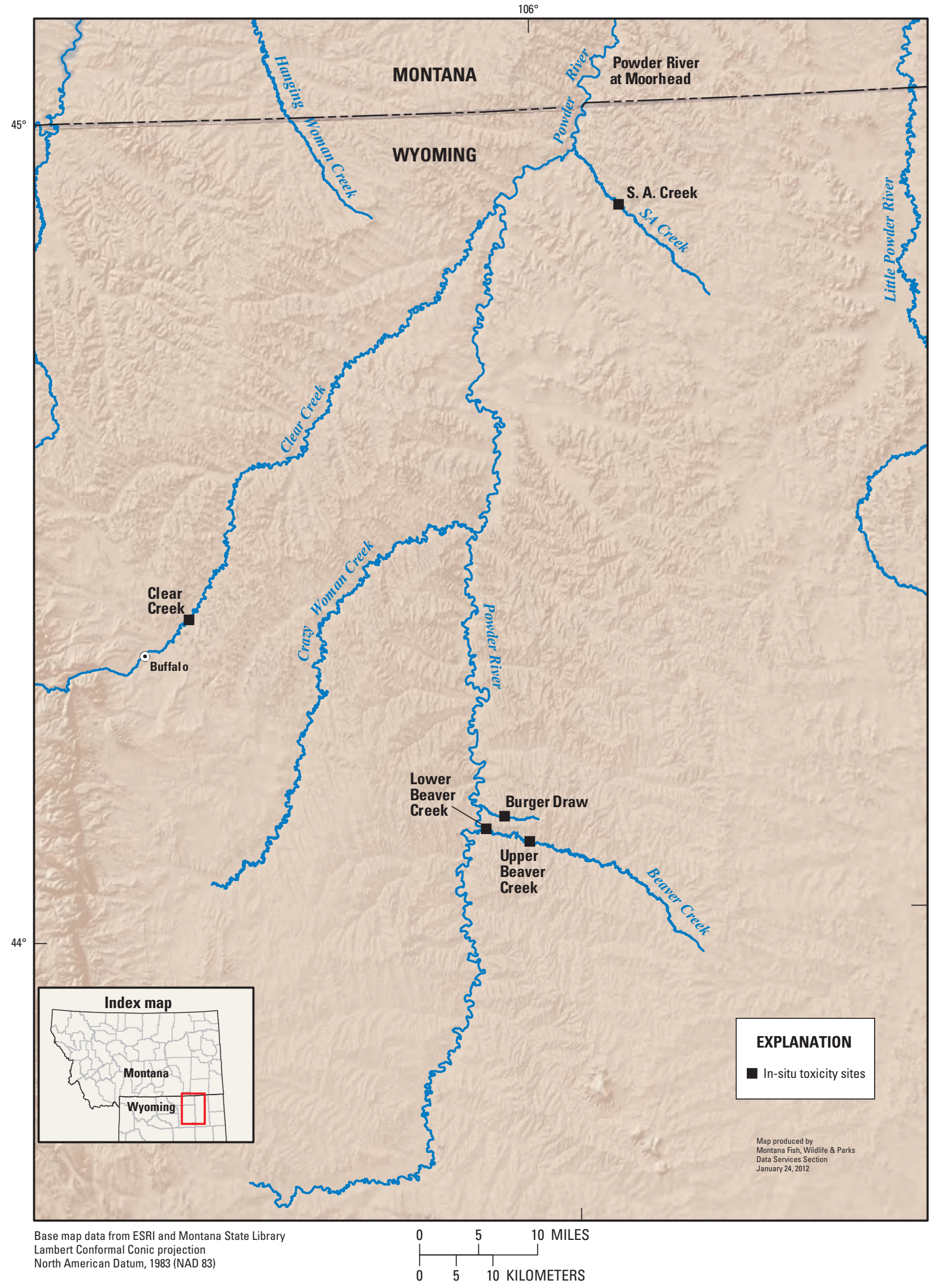

Figure 4-1. Field sites for 96-hour in situ toxicity experiments in the Powder River drainage, Wyoming. 
Table 4-2. Mean water chemistry recorded with hand-held meters during in situ toxicity experiments with fathead minnows (Pimephales promelas) in the Powder River Basin in Wyoming and Montana.

$\left[{ }^{\circ} \mathrm{C}\right.$, degrees Celsius; $\mu \mathrm{S} / \mathrm{cm}$, microsiemens per centimeter; $\mathrm{mg} / \mathrm{L}$, milligrams per liter; ( ), standard deviation error; $\mathrm{N}$, sample size]

\begin{tabular}{lcccc}
\hline \multicolumn{1}{c}{ Site } & $\begin{array}{c}\text { Temperature } \\
\left({ }^{\circ} \mathbf{C}\right)\end{array}$ & $\begin{array}{c}\text { Conductiv- } \\
\text { ity }(\boldsymbol{\mu S} / \mathbf{c m})\end{array}$ & $\begin{array}{c}\text { Dissolved } \\
\text { oxygen } \\
(\mathbf{m g} / \mathbf{L})\end{array}$ & $\mathbf{p H}$ \\
\hline $\begin{array}{c}\text { Powder R. at } \\
\text { Moorhead }\end{array}$ & 27.4 & 3,616 & 7.6 & 8.3 \\
& $(2.3)$ & $(131)$ & $(.5)$ & $(.2)$ \\
Clear Creek & $\mathrm{N}=5$ & $\mathrm{~N}=5$ & $\mathrm{~N}=5$ & $\mathrm{~N}=5$ \\
& 21.6 & 857 & 8.9 & 8.5 \\
& $(3.2)$ & $(49)$ & $(2.0)$ & $(.3)$ \\
Lower Beaver & $\mathrm{N}=9$ & $\mathrm{~N}=9$ & $\mathrm{~N}=9$ & $\mathrm{~N}=8$ \\
& 21.9 & 2,843 & 8.8 & 9.3 \\
& $(3.4)$ & $(82)$ & $(1.0)$ & $(.1)$ \\
Upper Beaver & $\mathrm{N}=9$ & $\mathrm{~N}=9$ & $\mathrm{~N}=9$ & $\mathrm{~N}=4$ \\
& 20.3 & 2,612 & 8.0 & 9.0 \\
& $(2.4)$ & $(38)$ & $(.08)$ & $(.1)$ \\
& $\mathrm{N}=9$ & $\mathrm{~N}=9$ & $\mathrm{~N}=9$ & $\mathrm{~N}=4$ \\
Burger Draw & 21.9 & 3,649 & 8.3 & 9.0 \\
& $(4.7)$ & $(30)$ & $(.6)$ & $(.1)$ \\
& $\mathrm{N}=9$ & $\mathrm{~N}=9$ & $\mathrm{~N}=9$ & $\mathrm{~N}=6$ \\
\hline SA Creek & 27.6 & 2,620 & 9.5 & 8.9 \\
& $(2.5)$ & $(91)$ & $(2.0)$ & $(.2)$ \\
& $\mathrm{N}=6$ & $\mathrm{~N}=6$ & $\mathrm{~N}=6$ & $\mathrm{~N}=6$ \\
\hline
\end{tabular}

Table 4-3. Minimum and maximum temperature, conductivity, dissolved oxygen, and $\mathrm{pH}$ measurements recorded every 30 minutes at four sites during in situ experiments with fathead minnows (Pimephales promelas) in the Powder River Basin, Wyoming and Montana. Data was recorded with YSI $5000^{\text {TM }}$ Data Sonde units.

$\left[{ }^{\circ} \mathrm{C}\right.$, degrees Celsius; $\mu \mathrm{S} / \mathrm{cm}$, microsiemens per centimeter; $\mathrm{mg} / \mathrm{L}$, milligrams per liter; NA, not applicable]

\begin{tabular}{|ccccc}
\hline \multicolumn{1}{c}{ Site } & $\begin{array}{c}\text { Tempera- } \\
\text { ture } \\
\left({ }^{\circ} \mathbf{C}\right)\end{array}$ & $\begin{array}{c}\text { Conductiv- } \\
\text { ity }(\boldsymbol{\mu S} / \\
\mathbf{c m})\end{array}$ & $\begin{array}{c}\text { Dissolved } \\
\mathbf{0 x y g e n} \\
(\mathbf{m g} / \mathbf{L})\end{array}$ & $\mathbf{p H}$ \\
\hline Powder River at & $21.34-$ & $3,280-$ & NA & $7.74-$ \\
Moorhead & 31.20 & 4,142 & & 8.38 \\
\hline Clear Creek & $15.12-$ & $669-891$ & $5.59-$ & $7.81-$ \\
& 29.51 & & 12.33 & 8.73 \\
\hline Lower Beaver & $14.13-$ & $2,390-$ & $5.14-$ & $9.23-$ \\
& 31.95 & 2,644 & 10.68 & 9.35 \\
\hline \multirow{2}{*}{ SA Creek } & $16.21-$ & $2,095-$ & NA & $8.50-$ \\
& 29.97 & 2,892 & & 8.97 \\
\hline
\end{tabular}

site) Upper Beaver Creek, Lower Beaver Creek, and Burger Draw (experimental sites) with the same methods employed with FHM during 2006. The water quality monitoring design and methods were the same as described for 2006 in situ experiments (tables 4-5, 4-6, and 4-7); however, in addition to performing on site ammonia assays with a $\mathrm{Hach}^{\mathrm{TM}}$ test kit, water samples were collected and acidified for ammonia analyses in the laboratory with the U.S. Environmental Protection Agency 350.1 methodology (U.S. Environmental Protection Agency, 1993) (table 4-8).

In addition to the in situ experiments completed in the field in 2007, a simultaneous experiment was completed with site water in more controlled conditions. Water was collected at Clear Creek (reference site), Upper Beaver Creek, Lower Beaver Creek, and Burger Draw (experimental sites) and held in insulated 22-L containers. Four replicates of five fish were exposed to water from each site in $530-\mathrm{mL}$ containers. The containers were kept in a water bath that fluctuated slightly with the room temperature $\left(16-22^{\circ} \mathrm{C}\right)$, and water in the containers was replaced every $12 \mathrm{~h}$.

Water samples intended for laboratory analyses were kept refrigerated in Buffalo, Wyoming, and transported in ice to the U.S. Geological Survey, Columbia Environmental Research Center, Jackson Field Research Station, Jackson, Wyoming (from here defined as Jackson Field Research Station).

Samples were then sorted, and shipped in ice to the State of Montana Department of Health and Human Services Environmental Laboratory for analyses.

For both experiments in 2006, the survival data were analyzed with the Toxstat software package (Toxstat Version $3.4,1994)$. A T-test was performed between the reference sites (experiment 1). Because no difference was observed, the data were pooled and a one-way ANOVA was performed followed by a Bonferroni means comparison. Because one reference site was used during experiment 2 , no pooling was necessary. T-tests were used to compare the survival of 2-dph against 6-dph FHM at sites. All data met homogeneity and normality assumptions without transformations. In 2007, a one-way ANOVA was performed followed by a Bonferroni means comparison for 4-dph and 6-dph pallid sturgeon in situ, and 4-dph pallid sturgeon static-renewal experiments. All means were tested with a statistical criterion of alpha less than $(\alpha<) 0.05$.

Potential differences in mean ammonia concentrations derived with field assays of chilled samples and the EPA method 350.1 laboratory assays were defined with T-tests in the Toxstat software package with a statistical criterion of $\alpha<$ 0.05 . No transformations were necessary as all data met homogeneity and normality assumptions without transformation.

\section{Mixing Zone and Site Water}

Mixing zones were characterized at three locations of the Powder River/Tongue watersheds: downstream from the confluence of Beaver Creek and the Powder River; downstream from the untreated product water diffuser in the Tongue River 
Table 4-4. Additional trace metal scans analyzed with Inductively Coupled Plasma (ICP) in water samples collected from in situ experimental sites for fathead minnows (Pimephales promelas). Experiments completed in the Powder River Basin, Wyoming and Montana. Sample size is one for all constituents.

$\left[<\right.$, less than detection limit; $\mathrm{mg} / \mathrm{L}$, milligrams per liter; ( ), date sample was taken (month/day/year); $\mathrm{CaCO}_{3}$, calcium carbonate $]$

\begin{tabular}{lrrrr}
\hline \multicolumn{1}{c}{ Constituent (mg/L) } & $\begin{array}{c}\text { Powder River at } \\
\text { Moorhead (7/26/2006) }\end{array}$ & $\begin{array}{c}\text { Clear Creek } \\
(\mathbf{7 / 2 7 / 2 0 0 6 )}\end{array}$ & $\begin{array}{c}\text { Lower Beaver Creek } \\
(\mathbf{7 / 2 7 / 2 0 0 6 )}\end{array}$ & $\begin{array}{c}\text { Upper Beaver Creek } \\
(\mathbf{7 / 2 7 / 2 0 0 6 )}\end{array}$ \\
\hline Arsenic & $<0.001$ & $<0.001$ & 0.004 & 0.005 \\
Barium & .063 & .045 & .215 & .079 \\
Beryllium & $<.002$ & $<.002$ & $<.002$ & $<.002$ \\
Calcium & 150.000 & 88.800 & 10.600 & 14.600 \\
Cadmium & $<.002$ & $<.002$ & $<.002$ & $<.002$ \\
Chromium & .004 & .002 & .003 & .011 \\
Copper & .004 & .007 & .009 & .005 \\
Iron & .100 & .100 & .380 & .340 \\
Magnesium & 144.000 & 41.300 & 27.500 & 44.700 \\
Manganese & .048 & .052 & .011 & .031 \\
Sodium & 525.000 & 69.600 & 773.000 & 599.000 \\
Nickel & $<.010$ & $<.010$ & $<.010$ & $<.010$ \\
Lead & $<.005$ & $<.005$ & $<.005$ & $<.005$ \\
Selenium & $<.005$ & $<.005$ & $<.005$ & $<.005$ \\
Zinc & .011 & .052 & .058 & .008 \\
Total hardness as $\mathrm{CaCO}_{3}$ & 968.000 & 392.00 & 140.000 & 220.000 \\
\hline
\end{tabular}

Table 4-5. Mean alkalinity expressed as milligrams per liter calcium carbonate equivalent, bicarbonate, sulfate, calcium, magnesium, sodium, and potassium, during in situ experiments with 4- and 6-day-post-hatch pallid sturgeon (Scaphirhynchus albus) performed in the Powder River Basin in Wyoming.

[mg/L, milligrams per liter; ( ), standard error; N, sample size]

\begin{tabular}{|c|c|c|c|c|c|c|c|}
\hline Site & $\begin{array}{c}\text { Alkalinity (mg/L } \\
\text { as calcium } \\
\text { carbonate) }\end{array}$ & $\begin{array}{l}\text { Bicarbonate } \\
\text { (mg/L) }\end{array}$ & $\begin{array}{l}\text { Sulfate } \\
\text { (mg/L)) }\end{array}$ & $\begin{array}{l}\text { Calcium } \\
\text { (mg/L) }\end{array}$ & $\begin{array}{l}\text { Magnesium } \\
\text { (mg/L) }\end{array}$ & $\begin{array}{l}\text { Sodium } \\
\text { (mg/L) }\end{array}$ & $\begin{array}{l}\text { Potassium } \\
\text { (mg/L) }\end{array}$ \\
\hline \multirow[t]{3}{*}{ Clear Creek } & 145 & 165 & 322 & 84 & 36 & 58 & 5 \\
\hline & (17) & (16) & (41) & (8) & (3) & (6) & $(0.4)$ \\
\hline & $\mathrm{N}=5$ & $\mathrm{~N}=5$ & $\mathrm{~N}=5$ & $\mathrm{~N}=5$ & $\mathrm{~N}=5$ & $\mathrm{~N}=5$ & $\mathrm{~N}=5$ \\
\hline \multirow[t]{3}{*}{ Lower Beaver } & 1,419 & 1,513 & 289 & 16 & 35 & 681 & 19 \\
\hline & (55) & (59) & (33) & (1) & (1) & (17) & $(0.5)$ \\
\hline & $\mathrm{N}=4$ & $\mathrm{~N}=4$ & $\mathrm{~N}=4$ & $\mathrm{~N}=4$ & $\mathrm{~N}=4$ & $\mathrm{~N}=4$ & $\mathrm{~N}=4$ \\
\hline \multirow[t]{3}{*}{ Upper Beaver } & 1,468 & 1,626 & 163 & 16 & 29 & 650 & 20 \\
\hline & (26) & (11) & (10) & (1) & $(0.5)$ & (14) & $(0.5)$ \\
\hline & $\mathrm{N}=4$ & $\mathrm{~N}=4$ & $\mathrm{~N}=4$ & $\mathrm{~N}=4$ & $\mathrm{~N}=4$ & $\mathrm{~N}=4$ & $\mathrm{~N}=4$ \\
\hline \multirow[t]{3}{*}{ Burger Draw } & 2,348 & 2,561 & 56 & 14 & 23 & 943 & 29 \\
\hline & (47) & (67) & (11) & $(0.2)$ & $(0.5)$ & (17) & $(0.2)$ \\
\hline & $\mathrm{N}=5$ & $\mathrm{~N}=5$ & $\mathrm{~N}=5$ & $\mathrm{~N}=5$ & $\mathrm{~N}=5$ & $\mathrm{~N}=5$ & $\mathrm{~N}=5$ \\
\hline
\end{tabular}


Table 4-6. Mean temperature, conductivity, dissolved oxygen, and $\mathrm{pH}$ measurements recorded with hand-held meters during in situ toxicity experiments with pallid sturgeon (Scaphirhynchus albus) in the Powder River Basin in Wyoming.

$\left[{ }^{\circ} \mathrm{C}\right.$, degrees Celsius; $\mu \mathrm{S} / \mathrm{cm}$, microsiemens per centimeter; $\mathrm{mg} / \mathrm{L}$, milligrams per liter; ( ), standard deviation error; N, sample size]

\begin{tabular}{ccccc}
\hline Site & $\begin{array}{c}\text { Temperature } \\
\left({ }^{\circ} \mathbf{C}\right)\end{array}$ & $\begin{array}{c}\text { Conductivity } \\
(\boldsymbol{\mu S} / \mathbf{c m})\end{array}$ & $\begin{array}{c}\text { Dissolved } \\
\mathbf{0 x y g e n} \\
(\mathbf{m g} / \mathbf{L})\end{array}$ & $\mathbf{p H}$ \\
\hline Clear Creek & 24.6 & 747 & 8.9 & 8.9 \\
& $(.4)$ & $(53)$ & $(.4)$ & $(.1)$ \\
Lower Beaver & $\mathrm{N}=8$ & $\mathrm{~N}=7$ & $\mathrm{~N}=7$ & $\mathrm{~N}=8$ \\
& 23.5 & 2,803 & 7.1 & 9.0 \\
& $(1.8)$ & $(80)$ & $(.4)$ & $(.1)$ \\
Upper Beaver & $\mathrm{N}=6$ & $\mathrm{~N}=5$ & $\mathrm{~N}=5$ & $\mathrm{~N}=6$ \\
& 24.9 & 2,716 & 8.8 & 9.0 \\
& $(.6)$ & $(21)$ & $(.4)$ & $(.1)$ \\
Burger Draw & $\mathrm{N}=6$ & $\mathrm{~N}=5$ & $\mathrm{~N}=5$ & $\mathrm{~N}=6$ \\
& 22.2 & 3,553 & 7.8 & 8.9 \\
& $(.5)$ & $(158)$ & $(.2)$ & $(.1)$ \\
& $\mathrm{N}=8$ & $\mathrm{~N}=7$ & $\mathrm{~N}=7$ & $\mathrm{~N}=8$ \\
\hline
\end{tabular}

near Decker, Montana, not shown; and downstream from the treated product water diffuser (Higgins Loop ${ }^{\mathrm{TM}}$ Treatment) near Decker, Montana. Rhodamine WT dye was added at a constant drip into Beaver Creek and into the treated and untreated product water. At the Powder River, transects were spaced at 4, 8, 16, 32, and 804 meters $(\mathrm{m})$ below the confluence of Beaver Creek. On the Tongue River, transects were spaced 15, 30, 60, 90 and 1,200 m, downstream from the diffusers for treated and untreated discharge. The dye concentration was measured in the water at evenly spaced locations $(0.15-3 \mathrm{~m})$ across the river, and spacing was dependent on the width of the river. The number of locations across the width ranged from 5-11 locations and the width of the river at site locations ranged from 3 to $33.4 \mathrm{~m}$. At each point along the transect, dye concentrations were measured with a YSI-600 oms sonde ${ }^{\mathrm{TM}}$, equipped with a 6130 Rhodamine WT optical probe. The concentration of the injected dye solution was measured in reference to a dilution series of the stock solution (Kilpatrick and Cobb, 1985).

The depth of the mixing areas (shallow in the Powder River and deep in the Tongue River) and the complexity of the mixing action, made the logistics of completing an in situ toxicity experiment problematic. For this reason, it was decided to collect waters on site and complete site water experiments in the laboratory.

Table 4-7. Additional trace metal scans analyzed with Inductively Coupled Plasma (ICP) in water samples collected from in situ experimental sites with pallid sturgeon (Scaphirhynchus albus) completed in the Powder River Basin, Wyoming. Sample size is one for all constituents.

[mg/L, milligrams per liter; <, less than detection limit; ( ), date sample was taken (month/day/year); CaCO ${ }_{3}$, calcium carbonate]

\begin{tabular}{lcccc}
\hline \multicolumn{1}{c}{ Constituent (mg/L) } & $\begin{array}{c}\text { Clear Creek } \\
(\mathbf{7 / 1 1 / 2 0 0 6 )}\end{array}$ & $\begin{array}{c}\text { Burger Draw } \\
(\mathbf{7 / 1 1 / 2 0 0 6 )}\end{array}$ & $\begin{array}{c}\text { Lower Beaver Creek } \\
(\mathbf{7 / 1 1 / 2 0 0 6 )}\end{array}$ & $\begin{array}{c}\text { Upper Beaver Creek } \\
(\mathbf{7 / 1 1 / 2 0 0 6 )}\end{array}$ \\
\hline Arsenic & $<0.001$ & 0.001 & $<0.004$ & 0.004 \\
Barium & .04 & .220 & .080 & .170 \\
Beryllium & $<.001$ & .001 & $<.001$ & .001 \\
Calcium & 92.800 & 13.600 & 15.900 & 14.600 \\
Cadmium & $<.002$ & .002 & $<.002$ & .002 \\
Chromium & $<.002$ & .002 & $<.002$ & .002 \\
Copper & .001 & .001 & .006 & .180 \\
Iron & .140 & .130 & .830 & 28.400 \\
Magnesium & 40.400 & 22.300 & 34.800 & .006 \\
Manganese & .029 & .017 & .017 & 664.00 \\
Sodium & 72.400 & 986.000 & 71.000 & .010 \\
Nickel & $<.01$ & $<.010$ & $<.010$ & $<.0010$ \\
Lead & $<.001$ & $<.001$ & $<.001$ & $<.005$ \\
Selenium & $<.005$ & $<.005$ & $<.005$ & $<.005$ \\
\hline Zinc & .010 & .005 & .012 & 154.000 \\
Total hardness as CaCO & 398.000 & 126.00 & 183.00 & \\
\hline & & & & \\
\hline
\end{tabular}


Table 4-8. Mean concentrations of ammonia, in water from experimental sites during in situ experiments performed in the Powder River Basin, Wyoming. Measurements were performed with a Hach ${ }^{\mathrm{TM}}$ test kit in the field and with the EPA 350.1 methodology (U.S. Environmental Protection Agency, 1993) in the laboratory.

\begin{tabular}{lcc}
\hline \multicolumn{1}{c}{ Site } & $\begin{array}{c}\text { Ammonia } \\
\text { (mg/L, laboratory) }\end{array}$ & $\begin{array}{c}\text { Ammonia } \\
\text { (mg/ligrams field) }\end{array}$ \\
\hline Clear Creek & $0.01(0)$ & $0.01(0)$ \\
Lower Beaver & $\mathrm{N}=3$ & $\mathrm{~N}=5$ \\
& $.01(<.01)$ & $.4(.23)$ \\
Upper Beaver & $\mathrm{N}=3$ & $\mathrm{~N}=5$ \\
& $.12(.06)$ & $.29(.12)$ \\
Burger Draw & $\mathrm{N}=4$ & $\mathrm{~N}=4$ \\
& $1.05(.12)$ & $1.17(.07)$ \\
& $\mathrm{N}=5$ & $\mathrm{~N}=5$ \\
\hline
\end{tabular}

Experiments were completed with water collected from the Powder River and from Beaver Creek near the confluence with the Powder River, in Johnson County, Wyoming, not shown. Water also was collected from the Tongue River and two discharge points into the Tongue River in Big Horn County, Montana, not shown; one site contained treated (Higgins Loop ${ }^{\mathrm{TM}}$ ion exchange) coalbed natural gas $(\mathrm{CBNG})$ product water, the other site contained untreated $\mathrm{CBNG}$ product water; water was collected immediately above their discharge point into the Tongue River. Water collected from all sites was placed in acid washed, 20-L HDPE containers, which were chilled and transported to the Jackson Field Research Station. The waters were used to complete 96-h static renewal experiments with 2-dph FHM September-October, 2007.

Newly hatched FHM were shipped directly to the Jackson Field Research Station from Aquatic Biosystems, Fort Collins, Colorado. The brood stock were deemed free of disease and kept in isolation at the Colorado facility. The proper permits were obtained for importation of the offspring. At the Jackson Field Research Station, fish were acclimatized to Tongue River water and held at $20^{\circ} \mathrm{C}$.

Static-renewal experiments with the site waters were completed in 1-L glass beakers filled with $750 \mathrm{~mL}$ of site water and maintained in a water bath at $20^{\circ} \mathrm{C}$. The fish were exposed for 96-h and the experimental water was replaced every 24-h; the replacement water was allowed to equilibrate to the exposure temperature in the water bath overnight. Water from Beaver Creek, untreated CBNG product water, and treated CBNG product water were experimented with 100-percent, 75-percent, and 50-percent concentrations. Beaver Creek water was diluted with Powder River water to prepare the proper concentrations, and the product waters were diluted with Tongue River water to prepare the proper concentrations. Waters collected from the Powder and Tongue Rivers served as reference waters for the experiments. Water temperature and dissolved oxygen were monitored twice daily, and water samples were collected daily for water chemistry from the 100 percent (undiluted) and 50 percent diluted treatments (table 4-9).

\section{Results}

Ammonia concentrations were largest at Burger Draw and averaged $1.5-2.0 \mathrm{mg} / \mathrm{L}$ in 2006 , and $1.17 \mathrm{mg} / \mathrm{L}$ in 2007 . Ammonia concentrations did not exceed $0.5 \mathrm{mg} / \mathrm{L}$ at any other sites (table 4-8). Ammonia concentrations in samples measured in the field were greater than acidified samples measured in the laboratory. Differences were not significant between the methodologies at Burger Draw, Lower Beaver and Clear Creeks, however, differences were significant at Lower Beaver Creek $(\mathrm{T}=2.57)$.

Survival of 2-dph FHM in 2006 was 77 percent and 78 percent in the Powder River at Moorhead and the Clear Creek sites, respectively. Because there was no significant difference in survival between the two sites, the data were combined to create a pooled reference ( 78 percent as pooled reference) for further comparisons. Survival was significantly less at all experimental sites $(\mathrm{F}=2.77)$ when compared to the pooled reference (table 4-10) during experiment 1 . Survival of 6-dph FHM was 90 percent at Clear Creek (reference site) and was not significantly different at any experimental site when compared to the reference site.

Survival of 2-dph pallid sturgeon was low at all sites including the reference in 2007, and the experiment was terminated at $24 \mathrm{~h}$. The experiment was re-started with 4-dph fish, and survival at Clear Creek (reference site) was 95 percent, whereas survival in all experimental sites was 0 percent (table 4-11). Survival of 4-dph pallid sturgeon in a static-renewal experiment was similar to the in situ experiment, with reduced survival of fish exposed to product water (0 to 15 percent), and 90 percent survival of fish held in Clear Creek reference water. An experiment completed with 6-dph fish provided similar results when compared to the 4-dph FHM, though time to death was longer with the older fish.

\section{Site Water/Mixing Zone Experiments}

In the Powder River at the Beaver Creek confluence, dye concentrations, or otherwise stated as the part of the stream comprised of Beaver Creek discharge was greatest on the bank closest to the input source (table 4-12). At the first transect point, $0.3 \mathrm{~m}$ from the bank and $4 \mathrm{~m}$ below the confluence, the dye concentration was $122 \mu \mathrm{g} / \mathrm{L}$ or approximately 16 percent of the concentration of dye in undiluted Beaver Creek water. Dye concentrations across this transect $4 \mathrm{~m}$ below the confluence were; at $0.6 \mathrm{~m}, 106 \mu \mathrm{g} / \mathrm{L}$ or approximately 14 percent; at $0.9 \mathrm{~m}, 65 \mu \mathrm{g} / \mathrm{L}$ or approximately 8 percent; at 1.2 
Table 4-9. Mean alkalinity expressed as milligrams per liter calcium carbonate equivalent, bicarbonate, sulfate, calcium, magnesium, sodium, potassium, and total ammonia (mg N/L) during 96-hour static renewal exposures of 2-day-post-hatch fathead minnows (Pimephales promelas) to mixtures of waters collected in the Tongue and Powder River Basins, Wyoming and Montana, September 2007. Higgins Loop ${ }^{\text {TM }}$ technology was used to treat product water before release at one experimental location.

$\left[\mathrm{CaCO}_{3}\right.$, calcium carbonate; $\mathrm{mg} / \mathrm{L}$, milligrams per liter; ( ), standard error; $\mathrm{N}$, sample size; --, no standard deviation error when sample size is less than 3; NM, not measured]

\begin{tabular}{|c|c|c|c|c|c|c|c|c|}
\hline Site & $\begin{array}{c}\text { Alkalinity } \\
\left(\mathrm{CaCO}_{3^{\prime}} \text { in } \mathrm{mg} / \mathrm{L}\right)\end{array}$ & $\begin{array}{c}\text { Bicarbonate } \\
\text { (mg/L) }\end{array}$ & $\begin{array}{l}\text { Sulfate } \\
\text { (mg/L) }\end{array}$ & $\begin{array}{l}\text { Calcium } \\
\text { (mg/L) }\end{array}$ & $\begin{array}{l}\text { Magnesium } \\
\text { (mg/L) }\end{array}$ & $\begin{array}{l}\text { Sodium } \\
\text { (mg/L) }\end{array}$ & $\begin{array}{l}\text { Potassium } \\
\text { (mg/L) }\end{array}$ & $\begin{array}{c}\text { Total } \\
\text { ammonia } \\
\text { (mg N/L) }\end{array}$ \\
\hline \multirow{2}{*}{ Powder River } & (4) & (4) & $(37)$ & (5) & (3) & (18) & (1) & $(.06)$ \\
\hline & $\mathrm{N}=3$ & $\mathrm{~N}=3$ & $\mathrm{~N}=3$ & $\mathrm{~N}=3$ & $\mathrm{~N}=3$ & $\mathrm{~N}=3$ & $\mathrm{~N}=3$ & $\mathrm{~N}=3$ \\
\hline \multirow{2}{*}{ Tongue River } & (15) & (17) & $(493)$ & (2) & (16) & (17) & (.4) & -- \\
\hline & $\mathrm{N}=3$ & $\mathrm{~N}=3$ & $\mathrm{~N}=3$ & $\mathrm{~N}=3$ & $\mathrm{~N}=3$ & $\mathrm{~N}=3$ & $\mathrm{~N}=3$ & $\mathrm{~N}=2$ \\
\hline \multirow[t]{2}{*}{ Beaver Creek } & 1,233 & 1,097 & 354 & 13 & 29 & 553 & 16 & .16 \\
\hline & (76) & (34) & $(151)$ & -- & -- & -- & -- & -- \\
\hline Beaver Creek, 50 percent & $\mathrm{N}=2$ & $\mathrm{~N}=2$ & $\mathrm{~N}=2$ & $\mathrm{~N}=2$ & $\mathrm{~N}=2$ & $\mathrm{~N}=2$ & $\mathrm{~N}=2$ & -- \\
\hline \multirow[t]{3}{*}{ Treated } & 89 & 89 & 309 & 106 & 1.8 & 106 & 1.4 & .13 \\
\hline & $(2.1)$ & (2.1) & (24) & (3.3) & $(.5)$ & (9.9) & $(.1)$ & $(.02)$ \\
\hline & $\mathrm{N}=3$ & $\mathrm{~N}=3$ & $\mathrm{~N}=3$ & $\mathrm{~N}=3$ & $\mathrm{~N}=3$ & $\mathrm{~N}=3$ & $\mathrm{~N}=3$ & $\mathrm{~N}=3$ \\
\hline \multirow[t]{2}{*}{ Treated, 50 percent } & 199 & 195 & 221 & 81 & 16 & 63 & 2.8 & NM \\
\hline & $\mathrm{N}=2$ & $\mathrm{~N}=2$ & $\mathrm{~N}=2$ & $\mathrm{~N}=2$ & $\mathrm{~N}=2$ & $\mathrm{~N}=2$ & $\mathrm{~N}=2$ & -- \\
\hline \multirow[t]{3}{*}{ Untreated } & 1,560 & 1,420 & 97 & 9.7 & 5.6 & 678 & 8.5 & 2.0 \\
\hline & $(55)$ & $(25)$ & (23) & $(1.2)$ & (.8) & $(22)$ & (.4) & (.4) \\
\hline & $\mathrm{N}=3$ & $\mathrm{~N}=3$ & $\mathrm{~N}=3$ & $\mathrm{~N}=3$ & $\mathrm{~N}=3$ & $\mathrm{~N}=3$ & $\mathrm{~N}=3$ & $\mathrm{~N}=3$ \\
\hline
\end{tabular}

$\mathrm{m}, 60 \mu \mathrm{g} / \mathrm{L}$ or approximately 8 percent; at $1.5 \mathrm{~m}, 25 \mu \mathrm{g} / \mathrm{L}$ or approximately 3 percent; at $1.8 \mathrm{~m}, 13 \mu \mathrm{g} / \mathrm{L}$ or approximately 2 percent; at $2.1 \mathrm{~m}, 5 \mu \mathrm{g} / \mathrm{L}$ or $<1$ percent. At the first transect approximately 22 percent of the river water was considered mixed with Beaver Creek water although the concentrations vary across the width of the river at this point.

Dye concentrations at the transect $8 \mathrm{~m}$ below the confluence ranged from $58-2.5 \mu \mathrm{g} / \mathrm{L}$ or approximately $8-0.3$ percent of the concentration of dye in undiluted Beaver Creek water (table 4-12). At $8 \mathrm{~m}$ below the confluence approximately 40 percent of the stream was considered mixed with Beaver Creek water. At $16 \mathrm{~m}$ below the confluence of Beaver Creek, dye concentrations across the width of the river ranged from $48-4.4 \mu \mathrm{g} / \mathrm{L}$, or approximately $6-0.6$ percent of the concentration of dye in undiluted Beaver Creek water. At the $16 \mathrm{~m}$ transect, approximately 55 percent of the stream was mixed with Beaver Creek water. At $32 \mathrm{~m}$ below the confluence of Beaver Creek, dye concentrations across the width of the river ranged from 37 to less than $1 \mu \mathrm{g} / \mathrm{L}$, or approximately 5 to less than 0.1 percent of the concentration of dye in undiluted Beaver Creek water. At the $32 \mathrm{~m}$ below the confluence, approximately 75 percent of the stream was considered mixed with Beaver Creek water. At $804 \mathrm{~m}$ below the confluence of Beaver Creek, dye concentrations across the width of the river ranged from 23-7 $\mu \mathrm{g} / \mathrm{L}$, or approximately 3-1 percent of the concentration of dye in undiluted Beaver Creek water. At $804 \mathrm{~m}$ below the confluence, approximately 100 percent of the stream was considered mixed with Beaver Creek water.

At the untreated water outflow into Tongue River near Decker, Montana, dye concentrations $15 \mathrm{~m}$ downstream from the diffuser ranged from $55-0 \mu \mathrm{g} / \mathrm{L}$, and was most concentrated in measurements taken closest to the diffuser, whereas three measurements located farthest from the diffuser outfall contained no dyed product water (table 4-13). At the first transect the Tongue River water was considered approximately 30 percent mixed with untreated product water. At $30 \mathrm{~m}$ below 
Table 4-10. Survival of fathead minnows (Pimephales promelas) during 96-hour in situ experiments in the Powder River Basin, Wyoming and Montana. Two experiments were performed with fathead minnows of different ages. Fish were 2-day-post-hatch (dph) at the initiation of experiment 1 and were 6 -day-post-hatch at the initiation of experiment 2. Significant differences were defined between the pooled reference and experimental sites for experiment 1 and between Clear Creek (reference) and the experimental sites for experiment 2.

\begin{tabular}{|c|c|c|}
\hline Site & Age & $\begin{array}{c}\text { Percent } \\
\text { survival at } \\
96 \text { hours }\end{array}$ \\
\hline \multicolumn{3}{|c|}{ Experiment 1} \\
\hline Powder River at Moorhead & 2-dph & 77 \\
\hline Clear Creek & 2-dph & 78 \\
\hline Pooled Reference & 2-dph & 78 \\
\hline SA Creek & 2-dph & ${ }^{1} 24$ \\
\hline Burger Draw & 2-dph & ${ }^{1} 37$ \\
\hline Upper Beaver & 2-dph & ${ }^{1} 11$ \\
\hline Lower Beaver & 2-dph & ${ }^{1} 49$ \\
\hline \multicolumn{3}{|c|}{ Experiment 2} \\
\hline Clear Creek & 6-dph & 90 \\
\hline Burger Draw & 6-dph & 73 \\
\hline Upper Beaver & 6-dph & 75 \\
\hline Lower Beaver & 6-dph & 75 \\
\hline
\end{tabular}

the confluence, dye concentrations across the width of the river ranged from $39-0 \mu \mathrm{g} / \mathrm{L}$, and one of the transect points closest to the opposite stream bank contained no dyed product water. At $30 \mathrm{~m}$, the Tongue River water was considered approximately 47 percent mixed with untreated product water. At $60 \mathrm{~m}$, dye concentrations across the width of the river ranged from $25-0 \mu \mathrm{g} / \mathrm{L}$, and one transect point closest to the opposite stream bank contained no dyed product water. The Tongue River water was considered approximately 47 percent mixed with untreated product water at this point. At $90 \mathrm{~m}$, dye concentrations across the width of the Tongue River ranged from $23-3 \mu \mathrm{g} / \mathrm{L}$, and was considered approximately 67 percent mixed with the untreated product water. At 1,200 m, dye concentrations across the width of the river ranged from 19-6 percent and was considered approximately 100 percent mixed with untreated product.

At the treated water outflow, dye concentrations measured $15 \mathrm{~m}$ downstream from the diffuser ranged from $30-0 \mu \mathrm{g} / \mathrm{L}$, and the dye was most concentrated in the center of the stream channel near the diffuser outfall (table 4-14). Points along the width of the river that were nearest to both stream banks contained no dyed product water. This first transect was considered approximately 49 percent mixed with treated product water. At $30 \mathrm{~m}$, dye concentrations across the width of the Tongue River ranged from 37-0.6 $\mu \mathrm{g} / \mathrm{L}$. At $30 \mathrm{~m}$
Table 4-11. Survival of pallid sturgeon (Scaphirhynchus albus) during 96-hour in situ experiments in the Powder River Basin, Wyoming. Fish were 4 or 6-day-post-hatch (dph) at the initiation of the experiments. Significant differences were defined between Clear Creek (reference) and the experimental sites.

\begin{tabular}{|c|c|c|}
\hline Site & Age & $\begin{array}{l}\text { Percent survival at } \\
96 \text { hours }\end{array}$ \\
\hline \multicolumn{3}{|c|}{ In situ } \\
\hline Clear Creek & 4-dph & 95 \\
\hline Lower Beaver & 4-dph & ${ }^{1} 0$ \\
\hline Upper Beaver & 4-dph & ${ }^{1} 0$ \\
\hline Burger Draw & 4-dph & ${ }^{1} 0$ \\
\hline \multicolumn{3}{|c|}{ Static renewal } \\
\hline Clear Creek & 4-dph & 90 \\
\hline Lower Beaver & 4-dph & ${ }^{1} 10$ \\
\hline Upper Beaver & 4-dph & ${ }^{1} 15$ \\
\hline Burger Draw & 4-dph & ${ }^{1} 0$ \\
\hline \multicolumn{3}{|c|}{ In situ } \\
\hline Clear Creek & 6-dph & 80 \\
\hline Lower Beaver & 6-dph & ${ }^{1} 0$ \\
\hline Upper Beaver & 6-dph & ${ }^{1} 0$ \\
\hline Burger Draw & 6-dph & ${ }^{1} 0$ \\
\hline
\end{tabular}

below the confluence, the Tongue River water was considered approximately 49 percent mixed with treated product water. At $60 \mathrm{~m}$, dye concentrations across the width of the river ranged from $26-0 \mu \mathrm{g} / \mathrm{L}$, with one transect point closest to the opposite stream bank containing no dyed product water. At $60 \mathrm{~m}$ below the confluence, the Tongue River water was considered approximately 56 percent mixed with treated product water. At $90 \mathrm{~m}$, dye concentrations across the width of the river ranged from $26-6 \mu \mathrm{g} / \mathrm{L}$, and 1 transect point closest to the opposite stream bank contained no dyed product water. At $90 \mathrm{~m}$ below the confluence, the Tongue River water was considered approximately 66 percent mixed with untreated product water. At 1,200 m, dye concentrations across the width of the river ranged from 13-9 percent and at this point the Tongue River water was considered approximately 100 percent mixed with treated product water.

Survival of 2-dph FHM exposed to 100-percent Beaver Creek water and 100-percent untreated product water was reduced significantly when compared with Powder River and Tongue River reference sites. Survival of all other mixtures was not significantly different from the reference sites (fig. 4-2).

Ammonia concentrations were below U.S. Environmental Protection Agency water-quality criteria for all exposures and dilutions except the untreated CBNG product water (table 4-9; U.S. Environmental Protection Agency, 1999). Ammonia concentrations were great enough in the untreated product water (mean $=2.0 \mathrm{mg} / \mathrm{L})$ to contribute to mortality. 
Table 4-12. Dye concentrations as measured in transects across the Powder River downstream from the confluence of Beaver Creek. Beaver Creek entered on the right bank and distances at each transect were measured in meters from the right stream bank to the left stream bank.

[m, meter; $\mu \mathrm{g} / \mathrm{L}$, micrograms per liter; --, no measurment; <, less than]

\begin{tabular}{|c|c|c|c|c|c|c|c|c|c|}
\hline \multicolumn{2}{|c|}{ Transect 1 (4 m) } & \multicolumn{2}{|c|}{ Transect $2(8 \mathrm{~m})$} & \multicolumn{2}{|c|}{ Transect 3 (16 m) } & \multicolumn{2}{|c|}{ Transect $4(32 \mathrm{~m})$} & \multicolumn{2}{|c|}{ Transect $5(804 \mathrm{~m})$} \\
\hline $\begin{array}{l}\text { Distance } \\
\text { (m) }\end{array}$ & $\begin{array}{c}\text { Dye } \\
\text { ( } \mu g / L)\end{array}$ & $\begin{array}{l}\text { Distance } \\
\text { (m) }\end{array}$ & $\begin{array}{c}\text { Dye } \\
\text { ( } \mu \mathrm{g} / \mathrm{L})\end{array}$ & $\begin{array}{l}\text { Distance } \\
\text { (m) }\end{array}$ & $\begin{array}{l}\text { Dye } \\
\text { ( } \mu \mathrm{g} / \mathrm{L})\end{array}$ & $\begin{array}{c}\text { Distance } \\
\text { (m) }\end{array}$ & $\begin{array}{l}\text { Dye } \\
(\mu \mathrm{g} / \mathrm{L})\end{array}$ & $\begin{array}{l}\text { Distance } \\
\text { (m) }\end{array}$ & $\begin{array}{c}\text { Dye } \\
(\mu \mathrm{g} / \mathrm{L})\end{array}$ \\
\hline 0.3 & 122 & 0.2 & 55 & 0.3 & 47.7 & 3.0 & 37 & 6.1 & 17 \\
\hline .6 & 106 & .5 & 48 & .6 & 38.7 & 4.6 & 32 & 7.6 & 19 \\
\hline .9 & 65 & .8 & 38 & .9 & 33.8 & 6.1 & 23 & 9.1 & 22 \\
\hline 1.2 & 60 & 1.1 & 44 & 1.2 & 29.1 & 7.6 & 20 & 10.6 & 23 \\
\hline 1.5 & 25 & 1.4 & 58 & 1.5 & 26.4 & 9.1 & 13 & 12.2 & 22 \\
\hline 1.8 & 13 & 1.7 & 32 & 1.8 & 22.4 & 10.6 & 8 & 13.7 & 18 \\
\hline 2.2 & 5 & 1.0 & 12 & 2.1 & 10.1 & 12.2 & 4 & 15.2 & 16 \\
\hline-- & -- & 2.3 & 7 & 2.4 & 4.4 & 13.7 & 3 & 16.7 & 15 \\
\hline-- & -- & 2.6 & 2.5 & -- & -- & 18.2 & $<1$ & 18.2 & 12 \\
\hline-- & -- & -- & -- & -- & -- & -- & -- & 19.8 & 11 \\
\hline -- & -- & -- & -- & -- & -- & -- & -- & 21.3 & 7 \\
\hline
\end{tabular}

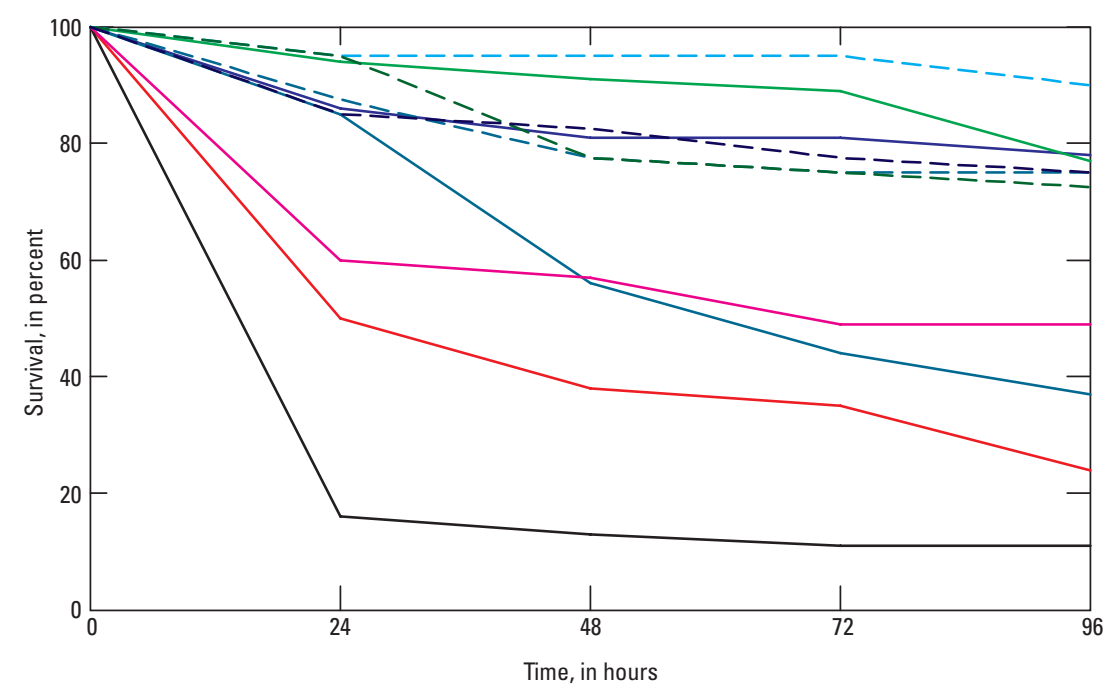

EXPLANATION

\begin{tabular}{ll}
\hline Clear Creek 2-day post hatch & - Lower Beaver Creek 2-day post hatch \\
Powder River 2-day post hatch & --- Clear Creek 6-day post hatch \\
SA Creek 2-day post hatch & --- Upper Beaver Creek 6-day post hatch \\
Upper Beaver Creek 2-day post hatch & -- - Burger Draw 6-day post hatch \\
\hline Burger Draw 2-day post hatch & --- Lower Beaver Creek 6-day post hatch
\end{tabular}

Figure 4-2. Percent survival at 96 hours for in situ experiments started with 2-daypost-hatch (2-dph) or 6-day-post-hatch (6-dph) fathead minnows. Powder River drainage, July-August 2006. 
Table 4-13. Dye concentrations as measured in transects across the Tongue River downstream from diffusers injecting untreated coal bed natural gas product water. Diffusers were located on the right bank and distances at each transect were measured in meters from the right stream bank to the left stream bank.

\begin{tabular}{|c|c|c|c|c|c|c|c|c|c|}
\hline \multicolumn{2}{|c|}{ Transect 1 (15 m) } & \multicolumn{2}{|c|}{ Transect $2(30 \mathrm{~m})$} & \multicolumn{2}{|c|}{ Transect $3(60 \mathrm{~m})$} & \multicolumn{2}{|c|}{ Transect $4(90 \mathrm{~m})$} & \multicolumn{2}{|c|}{ Transect $5(1,200 \mathrm{~m})$} \\
\hline $\begin{array}{l}\text { Distance } \\
\text { (m) }\end{array}$ & $\begin{array}{l}\text { Dye } \\
\text { ( } \mu \mathrm{g} / \mathrm{L})\end{array}$ & $\begin{array}{l}\text { Distance } \\
\text { (m) }\end{array}$ & $\begin{array}{l}\text { Dye } \\
\text { ( } \mu \mathrm{g} / \mathrm{L} \text { ) }\end{array}$ & $\begin{array}{l}\text { Distance } \\
\text { (m) }\end{array}$ & $\begin{array}{l}\text { Dye } \\
\text { ( } \mu \mathrm{g} / \mathrm{L})\end{array}$ & $\begin{array}{l}\text { Distance } \\
\text { (m) }\end{array}$ & $\begin{array}{l}\text { Dye } \\
\text { ( } \mu \mathrm{g} / \mathrm{L})\end{array}$ & $\begin{array}{l}\text { Distance } \\
\text { (m) }\end{array}$ & $\begin{array}{l}\text { Dye } \\
\text { ( } \mu \mathrm{g} / \mathrm{L})\end{array}$ \\
\hline 4.3 & 35 & 3.0 & 39 & 3.0 & 23 & 3.0 & 23 & 3.0 & 19 \\
\hline 10.3 & 30 & 6.1 & 22 & 6.1 & 25 & 6.1 & 21 & 6.1 & 16 \\
\hline 13.4 & 55 & 9.1 & 29 & 9.1 & 23 & 9.1 & 16 & 9.1 & 15 \\
\hline 16.4 & 0 & 12.2 & 10 & 12.2 & 13 & 12.2 & 9 & 12.2 & 12 \\
\hline 19.5 & 0 & 15.2 & 0 & 15.2 & 5 & 15.2 & 10 & 15.2 & 14 \\
\hline 22.5 & 0 & -- & -- & 18.2 & 0 & 18.2 & 14 & 18.2 & 11 \\
\hline -- & -- & -- & -- & -- & -- & 21.3 & 11 & 21.3 & 9 \\
\hline-- & -- & -- & -- & -- & -- & 24.3 & 13 & 24.3 & 9 \\
\hline-- & -- & -- & -- & -- & -- & 27.4 & 3 & 27.4 & 10 \\
\hline-- & -- & -- & -- & -- & -- & -- & -- & 30.4 & 8 \\
\hline-- & -- & -- & -- & -- & -- & -- & -- & 33.4 & 6 \\
\hline
\end{tabular}

Table 4-14. Dye concentrations as measured in transects across the Tongue River downstream from diffusers injecting treated coal bed natural gas product water. Diffusers were located on the right bank and distances at each transect were measured in meters from the right stream bank to the left stream bank.

[m, meter; $\mu \mathrm{g} / \mathrm{L}$, micrograms per liter; --, no measurment]

\begin{tabular}{|c|c|c|c|c|c|c|c|c|c|}
\hline \multicolumn{2}{|c|}{ Transect 1 (15 m) } & \multicolumn{2}{|c|}{ Transect $2(30 \mathrm{~m})$} & \multicolumn{2}{|c|}{ Transect $3(60 \mathrm{~m})$} & \multicolumn{2}{|c|}{ Transect $4(90 \mathrm{~m})$} & \multicolumn{2}{|c|}{ Transect $5(1,200 \mathrm{~m})$} \\
\hline $\begin{array}{l}\text { Distance } \\
\text { (m) }\end{array}$ & $\begin{array}{l}\text { Dye } \\
(\mu \mathrm{g} / \mathrm{L})\end{array}$ & $\begin{array}{l}\text { Distance } \\
\text { (m) }\end{array}$ & $\begin{array}{l}\text { Dye } \\
(\mu \mathrm{g} / \mathrm{L})\end{array}$ & $\begin{array}{l}\text { Distance } \\
\text { (m) }\end{array}$ & $\begin{array}{l}\text { Dye } \\
(\mu \mathrm{g} / \mathrm{L})\end{array}$ & $\begin{array}{l}\text { Distance } \\
\text { (m) }\end{array}$ & $\begin{array}{l}\text { Dye } \\
(\mu \mathrm{g} / \mathrm{L})\end{array}$ & $\begin{array}{l}\text { Distance } \\
\text { (m) }\end{array}$ & $\begin{array}{l}\text { Dye } \\
\text { ( } \mu \mathrm{g} / \mathrm{L} \text { ) }\end{array}$ \\
\hline 4.6 & 0 & 3.0 & 37 & 3.0 & 26 & 3.0 & 26 & 3.0 & 12 \\
\hline 6.1 & 0 & 6.1 & 32 & 6.1 & 25 & 6.1 & 24 & 6.1 & 9.5 \\
\hline 9.1 & 17 & 9.1 & 19 & 9.1 & 22 & 9.1 & 23 & 9.1 & 9 \\
\hline 12.2 & 18 & 12.2 & 12 & 12.2 & 17 & 12.2 & 18 & 12.2 & 13 \\
\hline 15.2 & 30 & 15.2 & 9 & 15.2 & 14 & 15.2 & 14 & 15.2 & 9.5 \\
\hline 18.2 & 0 & 18.2 & 3 & 18.2 & 10 & 18.2 & 12 & 18.2 & 13 \\
\hline 21.3 & 0 & 21.3 & .7 & 21.3 & 3 & 21.3 & 11 & 21.3 & 10 \\
\hline-- & -- & 24.3 & .6 & 24.3 & 2.8 & 24.3 & 10 & 24.3 & 9 \\
\hline -- & -- & -- & -- & 30.4 & 0 & 27.4 & 6 & -- & -- \\
\hline
\end{tabular}

\section{Discussion}

The value of in situ experiments as reviewed by Chappie and Burton (2000) cannot be understated for use in the Tongue and Powder River watersheds. The limitations of field experiments, including a lack of control for some physical variables, such as, temperature and turbidity, are precisely what give the experiments strong applicability. The daily fluctuations created by the diurnal cycle are a natural part of life in a prairie stream. The in situ experiments performed during this study were completed during mid-summer low-flow conditions, when days were long and fluctuations in temperature are most dramatic. Though care was taken to minimize lethal extremes (cover was provided and chambers were suspended in the water column), the natural cycle provided the setting that fish must endure in natural conditions.

The results of the field experiments support laboratory findings where fathead minnow and pallid sturgeon had reduced survival when exposed to elevated concentrations of $\mathrm{NaHCO}_{3}$. The concentrations of $\mathrm{NaHCO}_{3}$ in the various sites studied were above $1,800 \mathrm{mg} / \mathrm{L}$, a concentration above the determined lethal concentration that will cause 50 percent mortality or detrimental effect (LC50) of $1,718 \mathrm{mg} / \mathrm{L}$ and $1,493 \mathrm{mg} / \mathrm{L}$ for pallid sturgeon and fathead minnow, respectively (chapter 2, Results). Untreated product water was the dominant constituent of water at these sites and likely reduced survival. 
However, when the product water was treated with Higgins Loop ${ }^{\mathrm{TM}}$ technology, the acute toxicity of the water was removed. The Higgins Loop ${ }^{\mathrm{TM}}$ exchanges sodium for hydrogen ions (Severn Trent Services, Inc. 2010) and as a result, the $\mathrm{NaHCO}_{3}$ was reduced to less than $200 \mathrm{mg} / \mathrm{L}$. This reduction accounted for the reduced toxicity. It also should be noted that the concentration of ammonia was reduced after treatment from 2.0 to $0.13 \mathrm{mg} / \mathrm{L}$ (table 4-9). Acute toxicity is minimized by the Higgins Loop ${ }^{\text {TM }}$ treatment at the diffuser site near Decker, Montana.

Age and species sensitivity also were documented in response to $\mathrm{NaHCO}_{3}$ in the field. Newly hatched 2-dph fish were the most sensitive age for FHM, and mortality was significantly greater when compared to the reference sites. The same species exposed as 6-dph fish were less sensitive, and differences in survival between experimental and reference sites were not significant. Pallid sturgeon were more sensitive than FHM, and 0 percent survival occurred in both 4-dph and 6-dph pallid sturgeon at experimental sites. Therefore, pallid sturgeon had greater overall sensitivity to $\mathrm{NaHCO}_{3}$ (chapter 2 Results).

Johnson (2006) also completed in situ experiments with FHM during the summer of 2006. The researcher did not observe decreased survival at a site approximately 40 kilometers $(\mathrm{km})$ upstream from the Upper Beaver site used during this study, although concentrations of $\mathrm{NaHCO}_{3}$ were greater than the LC50 defined previously with FHM (Mount and others, 1992; chapter 2). However, Johnson used fish that were 12-dph to 14-dph. The Johnson results are similar to the lack of survival effects observed when 6-dph FHM were used in this study.

Others have documented age sensitivity to various contaminants (Rand, 1995), and U.S. Environmental Protection Agency (2002a) guidelines for the assessment of chronic toxicity suggest the use of FHM $<48 \mathrm{~h}$ old. However, current guidelines established in 2002 allow the use of FHM as old as 14-dph to assess acute toxicity (U.S. Environmental Protection Agency, 2002a, U.S. Environmental Protection Agency 2002b). Based on the results of this study, operators, managers and researchers may wish to take care when interpreting and comparing results gathered with various aged fish less than 14-dph.

Survival of 2-dph FHM exposed to site water collected from Beaver Creek in the laboratory was similar to that of FHM exposed in the field in 2006, with 40 percent surviving in controlled, laboratory conditions (fig. 4-3), and 49 percent surviving in the field study (table 4-10). The apparent sensitivity of 2-dph FHM compared with 6-dph FHM highlights the importance of experimenting with the most sensitive lifestages when evaluating the effects of toxicants.

Ammonia concentrations exceeded U.S. Environmental Protection Agency recommended maxima in Burger Draw in $2006(1.32 \mathrm{mg} / \mathrm{L}$ at $\mathrm{pH} 9.0)$ and may have contributed to mortality of FHM (U.S. Environmental Protection Agency, 1999).
Elevated concentrations of ammonia in Burger Draw also were documented by Smith and others (2009) when the researchers calculated lethal concentrations of ammonia in June 2004. Smith and others (2009) also cautioned about the existence of a diel cycle for ammonia where concentrations would be greatest in mid to late afternoon and the smallest in pre-dawn hours. Samples collected during this study were collected late morning to late afternoon and thus, generally should have documented the largest concentrations of the diel cycle.

At all other experimental sites, ammonia concentrations were not above chronic criteria concentrations, and survival statistically was reduced when compared to the reference site. This indicates that although ammonia may have played a part in the mortality at Burger Draw, other toxicants were the likely causative agent at all other experimental sites for 2-dph FHM, and 4-dph and 6-dph pallid sturgeon. To further support this conclusion, water samples collected from Beaver Creek had $0.16 \mathrm{mg} / \mathrm{L}$ of total ammonia during the 2007 mixing zone analyses (compared to $0.4 \mathrm{mg} / \mathrm{L}$ in the 2006 in situ experiment) and survival was decreased in the experiment completed with the 2007 water (table 4-9, fig. 4-3).

Ammonia concentrations were measured with a $\mathrm{Hach}^{\mathrm{TM}}$ test kit, and in the laboratory with the U.S. Environmental Protection Agency 350.1 methodology (U.S. Environmental Protection Agency, 1994) on water samples that had been collected and acidified for ammonia analyses. The concentrations were smaller in samples measured in the laboratory, with a significant difference between samples collected at Lower Beaver Creek (table 4-8). However the mean concentrations determined by both methodologies were below U.S. Environmental Protection Agency recommended maxima.

The mixing zone appeared to persist $804 \mathrm{~m}$ below the main stem of the Powder River and the confluence of Beaver Creek, and 1,200 $\mathrm{m}$ downstream from the discharge of untreated and treated product water into the Tongue River. However, the area containing concentrated untreated product water was limited to locations immediately downstream from the confluence of Beaver Creek in the Powder River, and the diffusers in the Tongue River. In the laboratory experiments with Tongue and Powder Rivers water, the toxicity of water from the untreated outflow and from Beaver Creek was reduced when the water was diluted to 75 percent of the source water with the appropriate receiving water (main stem Powder River or Tongue River). In the Powder River below Beaver Creek, and the Tongue River below the diffusers, the plume of water exceeding more than 75 percent produced water was limited during low-flow conditions. If the low volume of product water and the relatively large quantity of dilution water remain constant, the acute toxicity from individual discharges of product water will be limited. The effects of chronic exposures and the cumulative effects from multiple sources of product water would likely occur at concentrations lower than 75 percent product water, but were not addressed in these field experiments. 


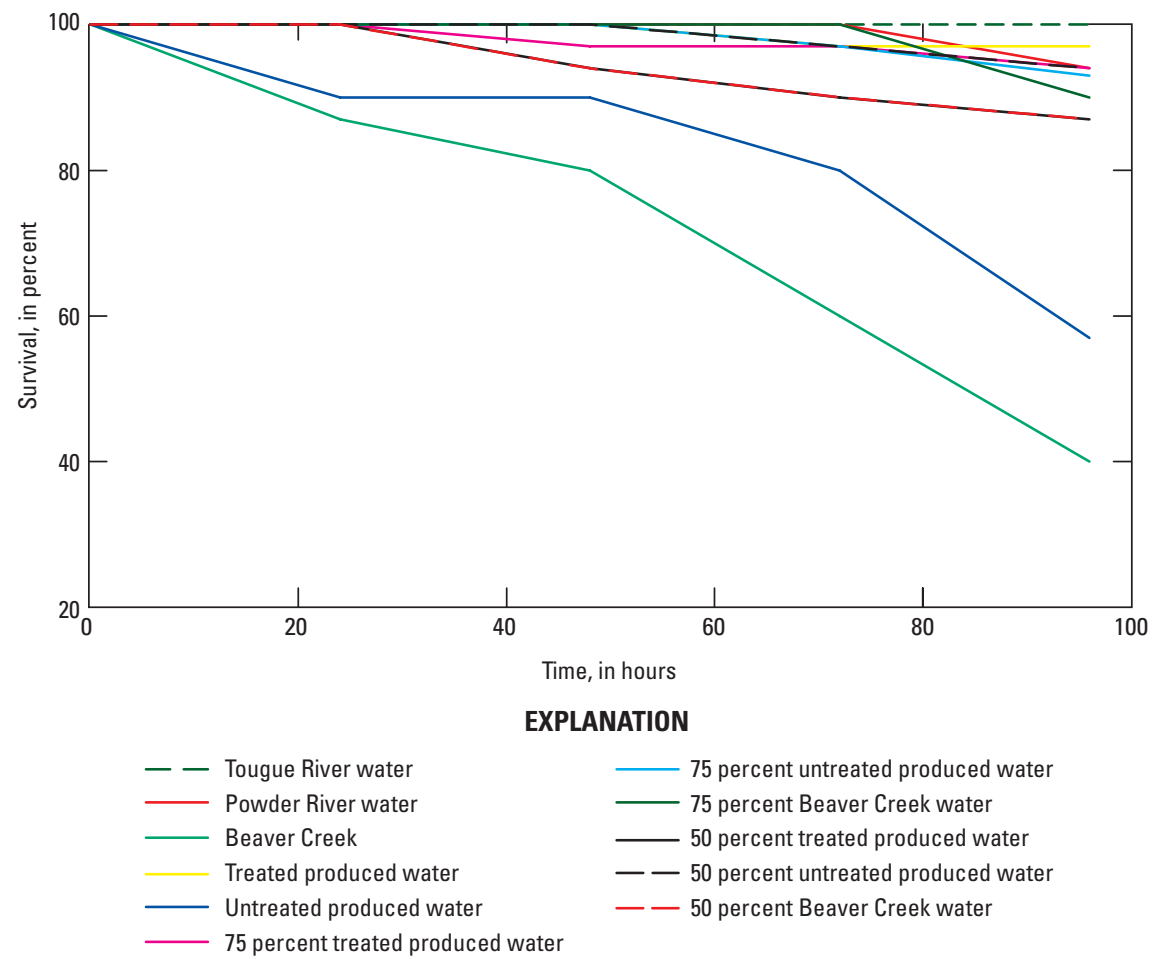

Figure 4-3. Percent survival at 96 hours for the mixing zone experiment started with 2-day-post-hatch fathead minnows. Receiving water was added to experimental waters to provide the percent mixture indicated. Sample waters were collected from the Powder and Tongue Rivers watershed, September-October 2007.

\section{Summary}

The combination of in situ experiments, static-renewal experiments performed simultaneously with in-situ experiments, and static renewal experiments performed with site water in the laboratory, demonstrated that coalbed natural gas (CBNG) product water reduces survival of fathead minnow (FHM) and pallid sturgeon. Age affected survival of FHM exposed to CBNG product water where fish 2-days-post-hatch (dph) were more sensitive than 6-dph fish, but pallid sturgeon survival was adversely affected at 4- and 6-dph. Ammonia likely contributed to toxicity in Burger Draw.

These results support the laboratory findings and LC50 determinations that will cause 50 percent mortality or detrimental effects defined in chapter 2, Results, and by Mount and others (1992). Therefore, the survival of early-lifestage fish, especially those $<6$-dph, likely is reduced significantly in the field when concentrations of $\mathrm{NaHCO}_{3}$ rise to more than $1,500 \mathrm{mg} / \mathrm{L}$.

The study results also determined that treatment with the Higgin's Loop ${ }^{\mathrm{TM}}$ technology and dilution of untreated water increased survival in the laboratory. It should be noted that both of these situations reduced ammonia in addition to concentrations of $\mathrm{NaHCO}_{3}$. Finally, the mixing zones of the three outfalls studied ranged from approximately $800-1,200 \mathrm{~m}$ below the confluence and the areas within these mixing zones with acutely lethal concentrations of $\mathrm{NaHCO}_{3}$ (as defined by the presence of concentrated dye) are limited and variable within each zone. These experiments addressed the acute toxicity of effluent waters being added to the main stem rivers, but did not address issues related to the volumes of water that may be added to the watershed.

\section{References Cited}

American Public Health Association Standard Methods, 1975a, Alkalinity titration method for water and wastewater, (14th ed.): Washington, D.C., American Public Health Association, p. 278.

American Public Health Association Standard Methods, 1975b, Hardness, Total, (14th ed.): Washington, D.C., American Public Health Association, p. 202.

Chappie, D.J., and Burton, G.A, 2000, Applications of aquatic and sediment toxicity testing In situ: Soil and Sediment Contamination, v. 9, p. 219-245. 
Farag, A.M., Skaar, D., Nimick, D.A., MacConnell, E., and Hogstrand, C., 2003, Characterizing aquatic health using salmonid mortality, physiology, and biomass estimates in streams with elevated concentrations of arsenic, cadmium, copper, lead, and zinc in the Boulder River Watershed, Montana: Transactions of the American Fisheries Society, v. 132 , p. $450-467$.

Harper, D.D., Farag, A. M., and Brumbaugh, W.G., 2008, Effects of acclimation on the toxicity of stream water contaminated with zinc and cadmium to juvenile cutthroat trout: Archives of Environmental Contamination and Toxicology, v. 54, p. 697-704.

Henry, T.B., Irwin, E. R., Grizzle, J. M., Brumbaugh, W. G., Wildhaber, M. L., 2001, Gill lesions and death of bluegill in an acid mine drainage mixing zone: Environmental Toxicology and Chemistry, v. 20, p. 1,304-1,311.

Johnson, Laurie A., 2006, Longitudinal changes in potential toxicity of coalbed natural gas produced water along Beaver Creek in the Powder River Basin, Wyoming: Laramie, University of Wyoming, Master's Thesis, $165 \mathrm{p}$.

Kilpatrick, F.A., and Cobb, E.D., 1985, Measurement of discharge using tracers: U.S. Geological Survey Techniques of Water-Resources Investigations, book 3, chap. A16, 52 p. (Also available at $h t t p: / / p u b s . u s g s . g o v / t w r i / t w r i 3-a 16 /$.

Patz, M. J., Reddy, K.J., and Skinner, Q.D., 2004, Chemistry of coalbed methane discharge water interacting with semiarid ephemeral stream channels: Journal of the American Water Resources Association, v. 40, no. 5, p. 1,247-1,255.

Rand, G.M., ed., 1995, Fundamentals of Aquatic Toxicology (2nd ed.): Philadelphia, Taylor and Francis, 1,125 p.

Severn Trent Services Inc, 2010, DOWEX ion exchange resins: Tampa, Severn Trent Services Inc, accessed April 25, 2010, at http://msdssearch. dow.com/PublishedLiteratureDOWCOM/ dh_0032/0901b803800325f0.pdf?filepath=liquidseps/pdfs/ noreg/177-02016.pdf\&fromPage $=$ GetDoc, $4 \mathrm{p}$.
Smith, R.L., Repert, D.A., and Hart, C.P., 2009, Geochemistry of inorganic water released from coalbed natural gas production wells in the Powder River Basin, Wyoming: Environmental Science and Technology, v. 43, p. 2,348-2,354.

Stewart, A.J., 1996, Ambient bioassays for assessing waterquality conditions in receiving streams: Ecotoxicology, v. 5, p. 377-393.

Toxstat Version 3.4, 1994, Cheyenne, Western Ecosystem Technology, Inc.

U. S. Environmental Protection Agency, 1993, Determination of ammonia nitrogen by semi-automated colorimetry, in O’Dell, J.W., ed., Method 350.1: Cincinnati, U. S. Environmental Protection Agency, $15 \mathrm{p}$.

U. S. Environmental Protection Agency, 1994, Short term methods for estimating the chronic toxicity of effluents and receiving waters to aquatic organisms, in Lewis, P.A., Klemm, D.J., Lazorchak, J.M., Norberg-King, T.J., Peltier, W.H., and Heber, M.A., eds., Duluth, U.S. Environmental Protection Agency, EPA/600/4 -91/002, 315 p.

U.S. Environmental Protection Agency, 1999, Water Quality Criteria: Washington, D.C., accessed April 2010 at www. epa.gov/waterscience/criteria/ammonia.

U.S. Environmental Protection Agency, 2002a, Short-term methods for estimating the chronic toxicity of effluents and receiving waters to aquatic organisms, (4th ed.): Washington, D.C., U.S. Environmental Protection Agency EPA821-R-02-13, 335 p.

U.S. Environmental Protection Agency, 2002b, Methods for measuring the acute toxicity of effluents and receiving waters to freshwater and marine organisms, (5th ed.): Washington, D.C., U.S. Environmental Protection Agency, EPA-821-R-02-012, 266 p. 


\section{Presence/Absence of Native and Non- Native Species of Fish in the Powder and Tongue River Basins}

By David D. Harper, Aïda M.Farag, Trevor Selch, and Don Skaar

Chapter 5 of

The Potential Effects of Sodium Bicarbonate, a Major Constituent of Produced Waters from Coalbed Natural Gas Production, on Aquatic Life

Edited by Aïda M. Farag and David D. Harper

Prepared in cooperation with Montana Fish, Wildlife, and Parks, U.S. Bureau of Land Management, and the U.S. Environmental Protection Agency

Scientific Investigations Report 2012-5008 


\section{Contents}

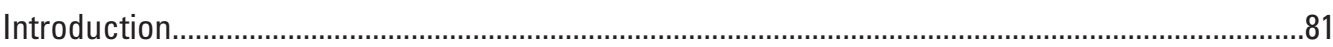

Methods

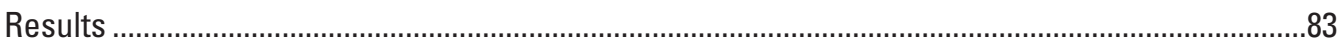

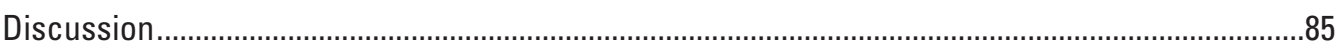

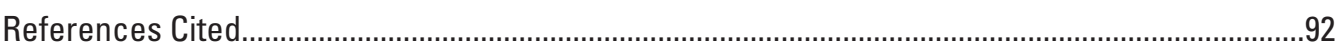

\section{Figures}

5-1. Map showing fish collection and habitat assessment sites in the Tongue and Powder Rivers, Wyoming and Montana .............................................................................82

5-2. Bar graph showing the number of sites where each fish species was collected in the Tongue and Powder River drainages, Wyoming and Montana..................................83

5-3. Pie diagram showing proportion of each fish species captured in the Tongue River drainage

5-4. Pie diagram showing proportion of each fish species captured in the Powder River drainage

\section{Tables}

5-1. Native fish species expected to occur and be present or absent, and non-native fish present at sites in the Tongue River drainage in Wyoming and Montana..

5-2. Native fish species expected to occur and present or absent, and non-native fish present at sites in the Tongue River drainage in Wyoming and Montana

5-3. Habitat characteristics associated with native fish distributions in the Tongue River watershed, Montana and Wyoming

5-4. Habitat characteristics associated with native fish distributions in the Powder River watershed, Montana and Wyoming

5-5. Habitat characteristics include the folowing; ability to withstand intermittent flow regime stream width, turbidity determined by the ability to see the bottom of a $3-\mathrm{ft}$ deep pool during baseflow conditions, substrate composition, presence of cover, presence of backwater/side-channels (including those with aquatic vegetation), and the absence of predators in a reach

5-6. Index of Biotic Integrity (IBI) (Bramblett and others 2005) scores for fish assemblages at Powder and Tongue River sampling locations in Wyoming and Montana 


\title{
Presence/Absence of Native and Non-Native Species of Fish in the Powder and Tongue River Basins
}

\author{
By David D. Harper', Aïda M.Farag', Trevor Selch², and Don Skaar ${ }^{2}$
}

\section{Introduction}

Before coalbed natural gas (CBNG) development and the associated increases of groundwater discharge, fisheries assessments in the Tongue and Powder River Basins were infrequent. Elser and others (1980), Baxter and Stone (1995), and Patton (1997) provided some of the earliest descriptions of species composition and habitat associations for species found in the Tongue and Powder River Basins. Clearwater and others (2002) suggested that an increase in salinity from product waters of CBNG production might increase salinity of the upper reaches of the Tongue River. They also suggested that an increase in discharge might affect the habitat quality of these reaches for fishes. Therefore, baseline data of species distribution are needed in these watersheds.

It also follows a study designed to document the presence or absence of the fish species that were used in experiments would provide valuable information about the current distribution of the test species in the field. It would be optimal to repeat the field investigations with time; however, a snapshot approach is not without merit. A one-time effort would provide information about the status of the species in question during that year. These data could then be used to alert researchers who may be involved in multiple-year efforts.

The composition and abundance of fish assemblages in the Tongue and Powder River Basins have been the focus of recent studies (Stagliano, 2006; Peterson and others, 2009; Peterson and others, 2010). Those studies were completed primarily to document and quantify the existing populations of resident and non-native fish species. The purpose of this study is to document the presence or absence of fathead minnow (Pimephales promelas) (FHM) and white sucker (Catostomus commersoni) to determine whether or not the selection of these species was appropriate for use in toxicity experiments (chapters 2, 3 and 4). Additionally, this research will provide a "snapshot" of the fish assemblage in the Tongue and Powder River Basins early into the development of CBNG.

${ }^{1}$ USGS, Columbia Environmental Research Center, Jackson Field Research Station, Jackson, Wyoming.

${ }^{2}$ Montana Fish, Wildlife, and Parks, Helena, Montana.

\section{Methods}

Fish and habitat surveys were completed in the Tongue and Powder River Basins July 19 through July 25, 2004. Fish and habitat measurements were taken in general conformity to U.S. Environmental Protection Agency Rapid Bioassessment protocols (Barbour and others, 1999). The method of fish capture typically was seining, with electrofishing used at a limited number of sites. Habitat measurements were modified to allow the data to be used in the Wyoming Warmwater Stream Assessment (WWSA) (Quist and others, 2006) and Montana Prairie Stream Index of Biotic Integrity (IBI) (Bramblett and others, 2005).

The WWSA protocol was used to predict the presence or absence of a species at a site based on historic distribution and physical habitat. The absence of a species at a site may be related to poor physical habitat rather than high salinity. The WWSA approach allows qualification of the "absence" side of the equation.

The framework for the WWSA assessment is described by Quist and others (2005), and Quist and others (2006). These methods provide information on the probable historic native fish distribution in a stream reach, which can be compared to the current (or sampled) native fish assemblage. An understanding of the expected fish assemblage in a stream reach can be used to evaluate factors that may be acting on the current fish assemblage. The comparison of predicted to observed fish assemblages can indicate changes in community structure because of natural or anthropogenic effects (Lipsey, 2001). For example, if a fish species is predicted in a stream reach based on its historic distribution, along with the elevation, stream size, and habitat requirements, but is not represented in a sample of the fish community; other factors may contribute to the distribution of that species (for example, water quality, predators, land management).

Because fish often have specific habitat requirements, their occurrence can be predicted based on the presence of habitat characteristics in a stream reach. Lee and others (1980), Holton (1990), and Baxter and Stone (1995) were used as sources for measurements of general habitat characteristics required and preferred by native fishes in the Powder and Tongue Rivers. 


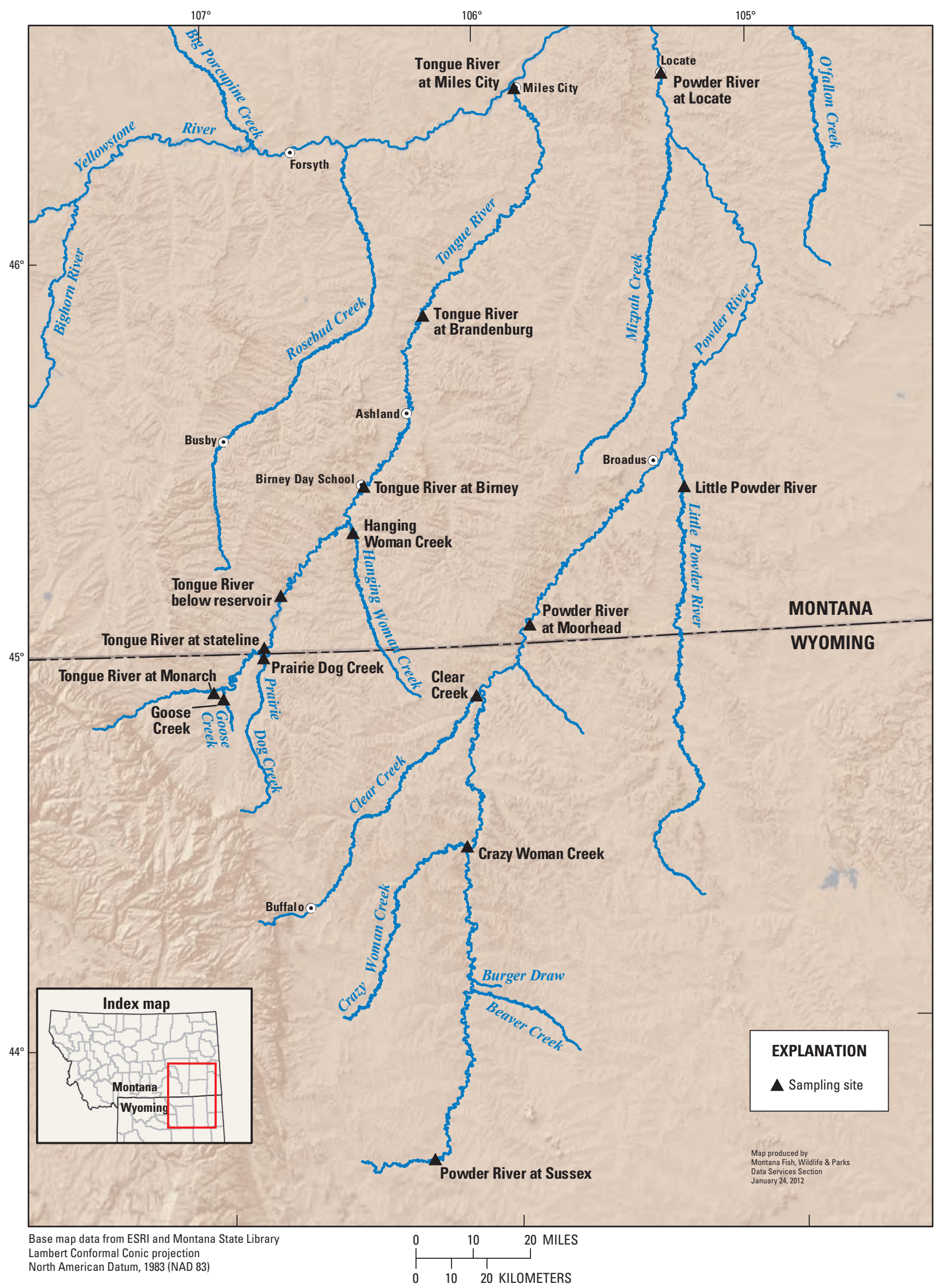

Figure 5-1. Fish collection and habitat assessment sites in the Tongue and Powder Rivers, Wyoming and Montana. 


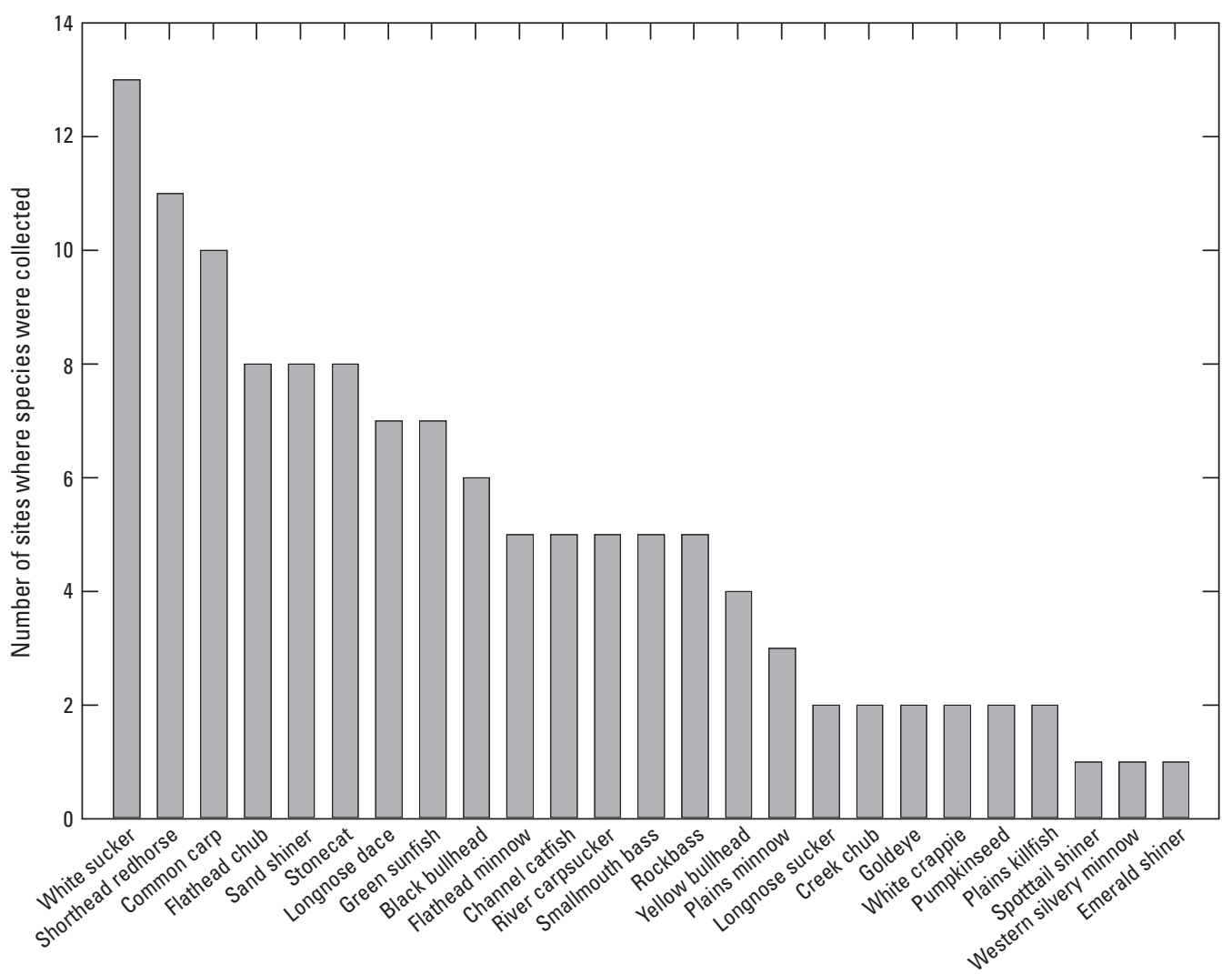

Figure 5-2. The number of sites where each fish species was collected in the Tongue and Powder River drainages, Wyoming and Montana. Total number of sample sites was 15.

Stream reaches were evaluated based on conformity between the habitat requirements of native fishes and the habitat characteristics in the stream reach. This information can be used to predict what fishes may be expected in a stream reach. As appropriate, the IBI scores were used to help interpret the presence/absence analyses. Sites with relatively small IBI scores typically are interpreted to have native fish assemblages that are affected by anthropogenic influences such as habitat, water-quality degradation and the presence of non-native fish species.

\section{Results}

Fisheries assessments were performed at 15 sites in the Tongue and Powder River Basins. In the Tongue River drainage, six sites were sampled in the main stem and three sites in major tributaries. In the Powder River drainage, six sites were sampled with three in the main stem, and three in major tributaries (fig. 5-1). At all sites combined, 17 native species and 8 non-native species were collected and a total of 4,620 fish were sampled. White sucker, shorthead redhorse, and common carp were collected at the most sites $(13,11$ and 10 , respectively; fig. 5-2). When data from both drainages were combined, the numbers of fish collected were greatest for sand shiners, flathead chub, and white suckers and were $(2,221$, 715 , and 371 , respectively).

In the Tongue River drainage, 16 native and 7 non-native species were collected (table 5-1; fig. 5-3). The number of species ranged from 12 on the Tongue River at Miles City; Tongue River at Brandenburg; and Tongue River at Stateline to 4 at Prairie Dog Creek. Number of individuals sampled at each site ranged from 262 at the Tongue River at Miles City to 23 on the Tongue River below the reservoir (because of limited sampling). White sucker, carp and stonecat were collected at the most sites (eight of nine sites). In terms of fish numbers collected in the Tongue River drainage; white suckers, rock bass and flathead chub occurred in the greatest numbers with 326,150 , and 139 , respectively.

In the Powder River drainage, a total of 13 native species and 5 non-native species were found (table 5-2; fig. 5-4). The number of fish species was greatest in Clear Creek (12 species) and smallest in the Powder River at Locate; Powder River at Moorhead; and Powder River at Sussex, (5 species). Sand shiners, flathead chub and white suckers were collected at the most number of sites in the Powder River drainage (6, 6, and 5 sites, respectively). Sand shiners were the most frequently collected species. Total number of individuals was greatest at Crazy Woman Creek, $(1,216)$ and smallest at Clear Creek, (221). 
Table 5-1. Native fish species expected to occur and be present or be absent, and non-native fish present at sites in the Tongue River drainage in Wyoming and Montana.

[( ), number of fish collected]

\begin{tabular}{|c|c|c|c|}
\hline Site & Present & Expected/absent & Present/non-native \\
\hline \multirow{4}{*}{$\begin{array}{l}\text { Tongue River Below Reservoir } \\
\text { (TRBR) }\end{array}$} & Brown bullhead (1) & & Carp (4) \\
\hline & White sucker (1) & & Green sunfish (6) \\
\hline & Yellow bullhead (3) & & Smallmouth bass (3) \\
\hline & River carpsucker (1) & & \\
\hline \multirow[t]{5}{*}{ Tongue River at Monarch (TRM) } & Shorthead redhorse (2) & Mountain sucker & Green sunfish (1) \\
\hline & Longnose sucker (1) & Fathead minnow & Rock bass (81) \\
\hline & Longnose dace (1) & White sucker & Common carp (1) \\
\hline & Stonecat (2) & & \\
\hline & Black bullhead (1) & & \\
\hline \multirow{8}{*}{$\begin{array}{l}\text { Tongue River at MT/WY state- } \\
\text { line (TRSL) }\end{array}$} & Black bullhead (5) & Lake chub & Rock bass (26) \\
\hline & White sucker (10) & Longnose dace & Spottail shiner (2) \\
\hline & Shorthead redhorse (1) & Fathead minnow & Smallmouth bass (5) \\
\hline & Stonecat (2) & Channel catfish & White crappie (33) \\
\hline & Yellow bullhead (12) & Goldeye & Common Carp (4) \\
\hline & & River carpsucker & \\
\hline & & Sauger & \\
\hline & & Emerald shiner & \\
\hline \multirow{7}{*}{$\begin{array}{l}\text { Tongue River at Birney Bridge } \\
\text { (TRBB) }\end{array}$} & White sucker (156) & Lake Chub & \\
\hline & Shorthead redhorse (32) & Mountain sucker & \\
\hline & Yellow bullhead (5) & Longnose dace & \\
\hline & Stonecat (1) & Fathead minnow & \\
\hline & Longnose dace (1) & River carpsucker & \\
\hline & & Sauger & \\
\hline & & Brassy minnow & \\
\hline \multirow{7}{*}{$\begin{array}{l}\text { Tongue River at Birney Bridge } \\
\text { (TRBB) }\end{array}$} & White sucker (156) & Lake chub & Smallmouth bass (2) \\
\hline & Shorthead redhorse (32) & Mountain sucker & Rockbass (4) \\
\hline & Yellow bullhead (5) & Longnose dace & Common carp (5) \\
\hline & Stonecat (1) & Fathead minnow & \\
\hline & Longnose dace (1) & River carpsucker & \\
\hline & & Sauger & \\
\hline & & Brassy minnow & \\
\hline \multirow{10}{*}{$\begin{array}{l}\text { Tongue River at Brandenburg } \\
\text { Bridge (TRBR) }\end{array}$} & Channel catfish (1) & Lake chub & Green sunfish (1) \\
\hline & White sucker (109) & Goldeye & Common carp (23) \\
\hline & Flathead chub (6) & Sauger & \\
\hline & Longnose dace (2) & Shovelnose sturgeon & \\
\hline & Sand shiner (4) & & \\
\hline & Shorthead redhorse (7) & & \\
\hline & River carpsucker (3) & & \\
\hline & Yellow bullhead (3) & & \\
\hline & Fathead minnow (11) & & \\
\hline & Stonecat (1) & & \\
\hline
\end{tabular}


Table 5-1. Native fish species expected to occur and be present or absent, and non-native fish present at sites in the Tongue River drainage in Wyoming and Montana.-Continued

[( ), number of fish collected]

\begin{tabular}{|c|c|c|c|}
\hline Site & Present & Expected/absent & Present/non-native \\
\hline \multirow[t]{5}{*}{ Goose Creek (GCR) } & Shorthead redhorse (2) & Lake chub & Smallmouth bass (3) \\
\hline & White sucker (4) & Mountain sucker & Common carp (7) \\
\hline & Stonecat (20) & Longnose dace & Rock bass (39) \\
\hline & Black bullhead (4) & Longnose sucker & \\
\hline & Fathead minnow (1) & & \\
\hline \multirow[t]{3}{*}{ Prairie Dog Creek (PDCR) } & White sucker (10) & Lake chub & White crappie (3) \\
\hline & Creek chub (6) & Stonecat & \\
\hline & Shorthead redhorse (1) & Goldeye & \\
\hline \multirow{3}{*}{$\begin{array}{l}\text { Hanging Woman Creek } \\
\text { (HWCR) }\end{array}$} & Fathead minnow (123) & Lake chub & Green sunfish (24) \\
\hline & Black bullhead (2) & Yellow bullhead & Common carp (79) \\
\hline & White sucker (28) & Brassy minnow & \\
\hline \multirow{11}{*}{$\begin{array}{l}\text { Tongue River at Miles City } \\
\text { (TRMC) }\end{array}$} & Western silvery minnow (53) & Fathead minnow & Common carp (4) \\
\hline & Stone cat (24) & Black bullhead & \\
\hline & Flathead chub (133) & Plains minnow & \\
\hline & White sucker (8) & Sauger & \\
\hline & Longnose dace (2) & Yellow bullhead & \\
\hline & Shorthead redhorse (2) & & \\
\hline & Sand shiner (27) & & \\
\hline & Emerald shiner (1) & & \\
\hline & River carpsucker (1) & & \\
\hline & Channel catfish (6) & & \\
\hline & Goldeye (1) & & \\
\hline
\end{tabular}

Habitat assessments completed during fish collections are summarized in tables 5-3, 5-4 and 5-5. IBI scores were calculated for 14 sites (table 5-6).

\section{Discussion}

White suckers and fathead minnows frequently were captured in the Tongue and Powder River Basins. White suckers were the most common species captured in terms of distribution (found at 13 of 15 sites) and numbers of fish collected in the Tongue River drainage. Whereas the proportion of sample sites with white suckers was not as large in their study, Peterson and others $(2009,2010)$ documented white suckers to be relatively abundant and widespread in the Tongue and Powder River drainages. This differs from Stagliano (2006), who indicated white suckers were not collected in the main stem Powder River at six sites in Montana. However, all sites sampled by Stagliano (2006) were in the main stem Powder River where white sucker are less numerous. Both this study and Peterson and others (2009 and 2010) documented that that the main stem Powder River had less abundance and frequency of occurrence of white sucker when compared to tributary sampling sites.

Fathead minnows were not as common as white suckers, but were collected at 5 of 15 sites ( 3 in the Tongue River drainage, 2 in the Powder River drainage). They were captured more frequently in tributaries than main stem sites in the Tongue and Powder River drainages. This is similar to Peterson and others (2009 and 2010) who found FHM to be, relatively, common in Tongue and Powder River tributaries. Stagliano (2006) did not collect fathead minnow during surveys of the Middle Powder River in Montana.

The presence of white suckers and FHM in tributary streams supports their selection for use as experimental organisms in acute and chronic toxicity experiments. Produced water entering the main stem Tongue and Powder Rivers is rapidly diluted (chapter 4, Results). However, product water that enters small tributaries may remain at effect concentrations until diluted upon entering larger bodies of water. Therefore, exposure to elevated concentrations of dissolved salts from produced water, such as sodium bicarbonate, is most likely to occur in tributaries.

Fish assemblages in the Tongue and Powder River Basins were generally similar to the findings of Stagliano (2006), 


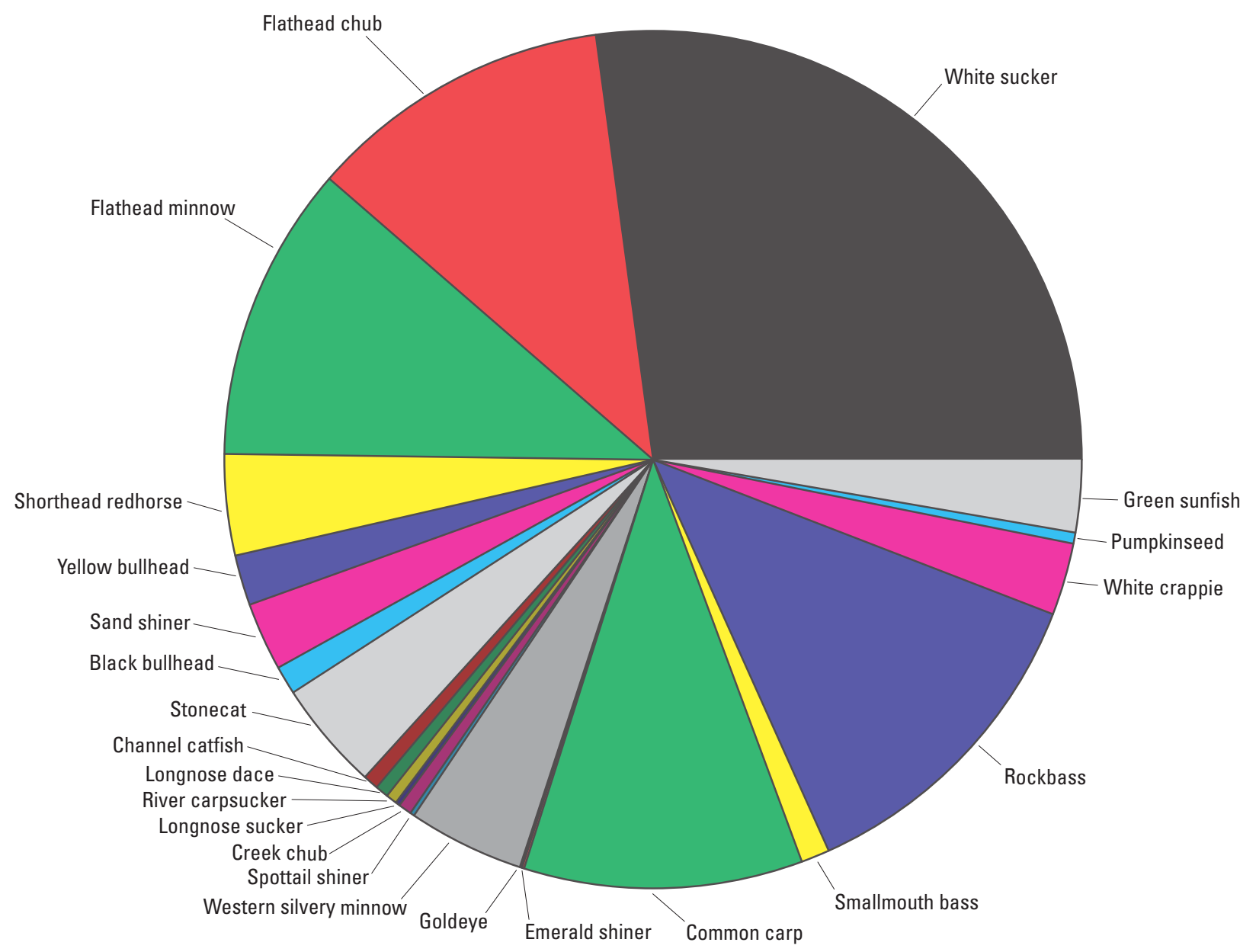

Figure 5-3. Proportion of each fish species captured in the Tongue River drainage. Fish were collected by seining and electrofishing .

Peterson and others (2009), and Peterson and others (2010). Although site locations were not identical, sand shiners were the most frequently collected species in the Powder River in all four studies. Generally, rock bass, smallmouth bass, common carp and green sunfish were present at more sites and in greater numbers in tributaries than in the main stem sites. The species composition in each study was slightly different, but these differences may be attributed to the general stochasticity of these systems. The Tongue River and Powder River are prairie streams draining watersheds with highly erodible soils, and are subject to extreme variations in discharge, which occur seasonally (from spring snow melt flow to extreme drought in the summer), and as a result of violent prairie thunderstorms with intense periods of precipitation (Poff and Ward, 1989). In addition, the underlying substrate of both streams is made up of, in large part, sand and fine sediments. The extreme variability and stochastic nature of the climate, combined with the unstable substrate underlying both streams has resulted in a fish community that is highly adaptable and variable from season to season (Grossman and others, 1982).

The Wyoming Warmwater Stream Assessment (WWSA) uses a series of physical assessments (for example, habitat measurements and sampling at representative sites), land use and historical data (for example, road density, irrigation withdrawal, reservoir construction) to predict the presence and absence of native fish species (Quist and others, 2005). The Montana Prairie Stream Index of Biotic Integrity (IBI) uses fish assemblage data to assess the condition of plains streams to assess anthropogenic disturbance (Bramblett and others, 2005). These attributes include but are not limited to the numbers of native and non-native species found at fish collection sites.

The WWSA and IBI models were in agreement at many sites. The Tongue River at Miles City and Crazy Woman Creek had large numbers of native fish species, low numbers of expected but absent species, and few non-native species. These sites also had the largest IBI scores. On the other end of the spectrum, Hanging Woman Creek, Tongue River at Stateline, and Powder River at Moorhead had few native fish species and many expected/absent and or non-native species, and these sites had the smallest IBI scores. At other sites, such as the Tongue at Brandenburg, Tongue River at Birney, Goose Creek, Tongue at Monarch, and the Little Powder River, the numbers of native species, expected/absent and present 
Table 5-2. Native fish species expected to occur and present or absent, and non-native fish present at sites in the Tongue River drainage in Wyoming and Montana.

[( ), number of fish collected]

\begin{tabular}{|c|c|c|c|}
\hline Site & Present & Expected/absent & Present/non-native \\
\hline \multirow[t]{10}{*}{ Powder River at Sussex (PRSX) } & Plains minnow (2) & Lake chub & Plains killifish (24) \\
\hline & Sand shiner (413) & Fathead minnow & \\
\hline & Flathead chub (31) & Burbot & \\
\hline & White sucker (5) & Black bullhead & \\
\hline & & Channel catfish & \\
\hline & & Goldeye & \\
\hline & & River carpsucker & \\
\hline & & Sauger & \\
\hline & & Sturgeon chub & \\
\hline & & Western silvery minnow & \\
\hline \multirow[t]{4}{*}{ Powder River at Locate (PRLM) } & White sucker (1) & Goldeye & Plains killifish (4) \\
\hline & Sand shiner (109) & & \\
\hline & Flathead chub (350) & & \\
\hline & Plains minnow (4) & & \\
\hline \multirow{5}{*}{$\begin{array}{l}\text { Powder River at Moorhead } \\
\text { (PRMD) }\end{array}$} & Sand shiner (37) & Goldeye & \\
\hline & Flathead chub (176) & & \\
\hline & Longnose dace (14) & & \\
\hline & Stonecat (12) & & \\
\hline & Channel catfish (7) & & \\
\hline \multirow[t]{8}{*}{ Clear Creek (CLCR) } & Sand shiner (138) & Lake chub & Rock bass (34) \\
\hline & Goldeye (2) & Longnose dace & Common carp (21) \\
\hline & White sucker (1) & Burbot & Green sunfish (4) \\
\hline & Stonecat (10) & & Smallmouth bass (1) \\
\hline & Shorthead redhorse (3) & & \\
\hline & Creek chub (3) & & \\
\hline & Flathead chub (2) & & \\
\hline & River carpsucker (2) & & \\
\hline \multirow[t]{7}{*}{ Crazy Woman Creek (CWCR) } & Sand shiner $(1,082)$ & Channel catfish & \\
\hline & Fathead minnow (16) & goldeye & \\
\hline & Black bullhead (3) & & \\
\hline & White sucker (6) & & \\
\hline & Longnose dace (35) & & \\
\hline & Flathead chub (13) & & \\
\hline & Plains minnow (61) & & \\
\hline \multirow[t]{8}{*}{ Little Powder River (LPR) } & River carpsucker (2) & Lake chub & Common carp (86) \\
\hline & Channel catfish (4) & Black bullhead & Green sunfish (6) \\
\hline & Shorthead redhorse (4) & Goldeye & \\
\hline & White sucker (30) & Yellow bullhead & \\
\hline & Flathead chub (4) & & \\
\hline & Longnose dace (1) & & \\
\hline & Sand shiner (411) & & \\
\hline & Fathead minnow (235) & & \\
\hline
\end{tabular}




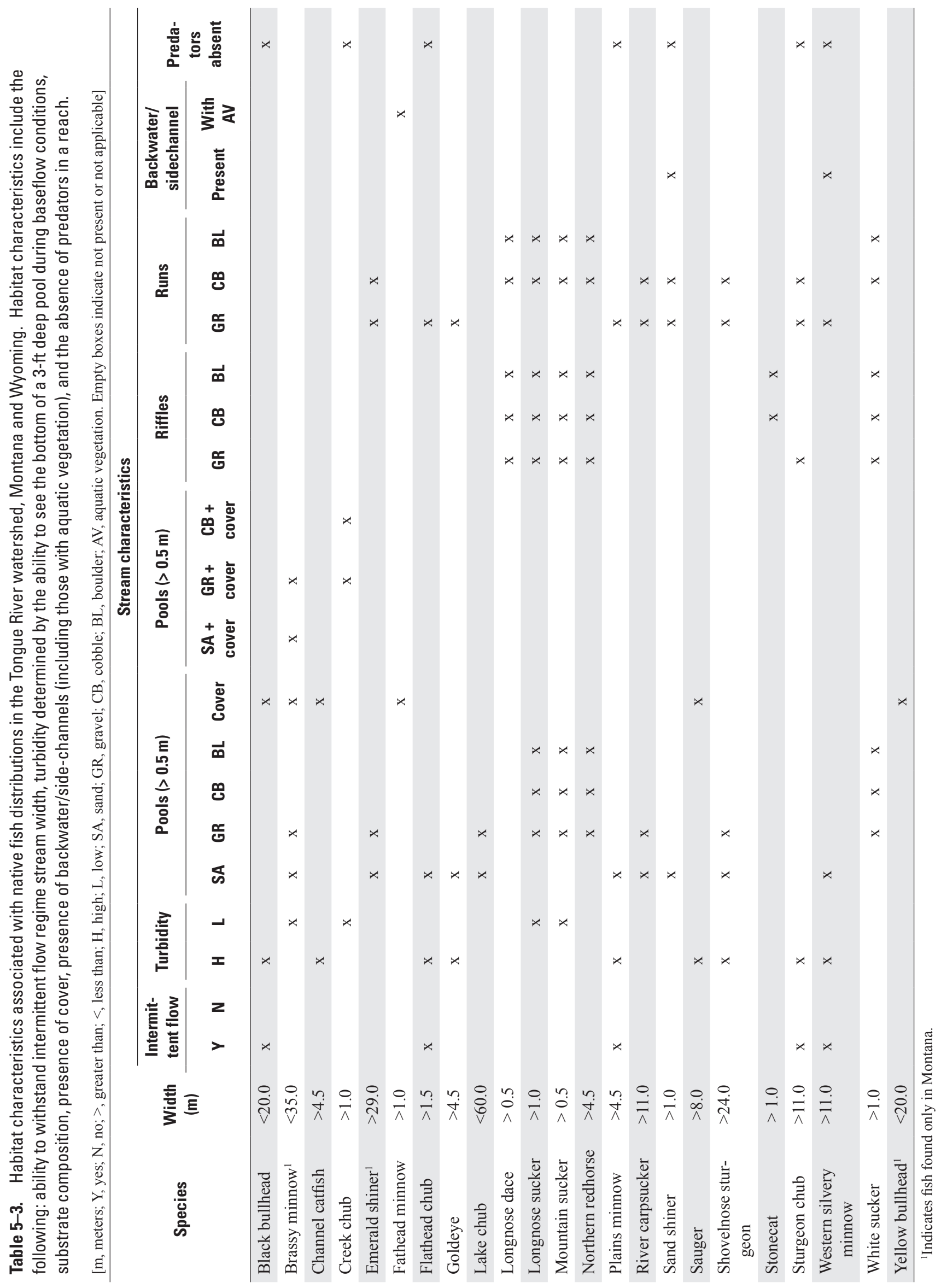




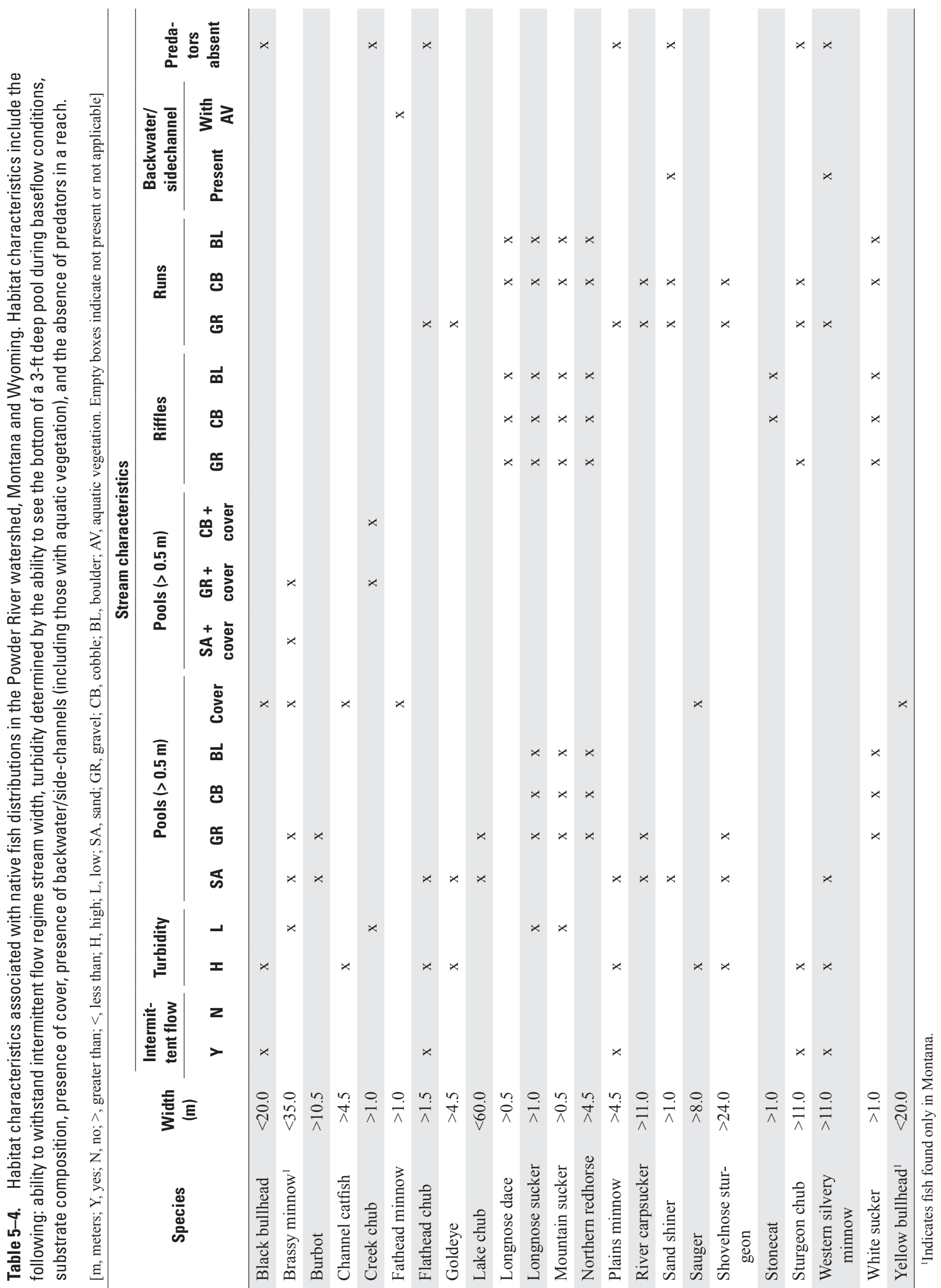




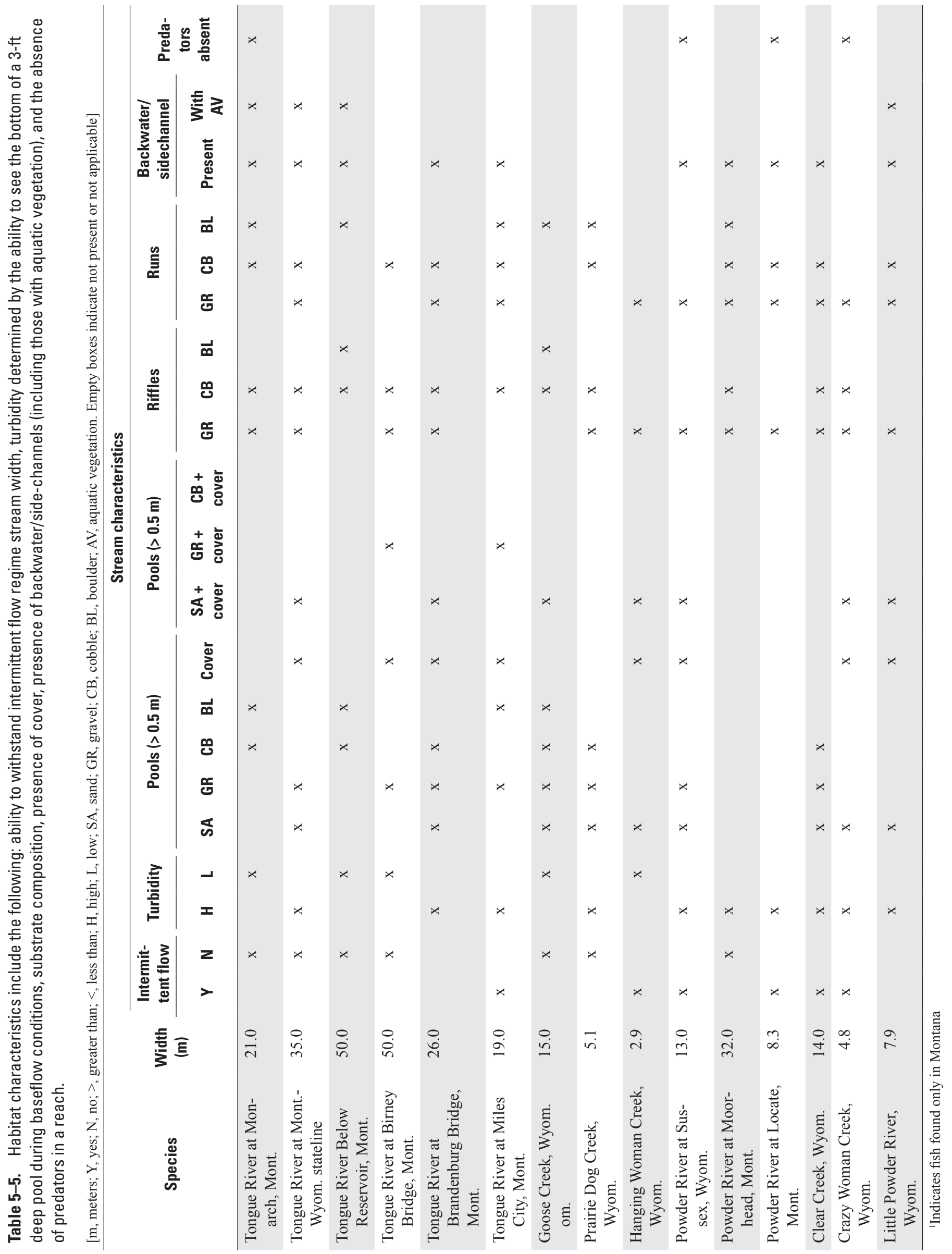


Table 5-6. Index of Biotic Integrity (IBI) (Bramblett and others 2005) scores for fish assemblages at Powder and Tongue River sampling locations in Wyoming and Montana.

$\left[\mathrm{km}^{2}\right.$, square kilometer; IBI, index of biotic integrity]

\begin{tabular}{lcc}
\hline \multicolumn{1}{c}{ River/section } & Drainage area $\left.\mathbf{( k m}^{\mathbf{2}}\right)$ & IBI score \\
\hline Tongue River at Miles City, Mont. & 13,998 & 63 \\
\hline Tongue River at Brandenburg Bridge, Mont. & 10,041 & 52 \\
Tongue River near Birney, Mont. & 6,824 & 53 \\
\hline Hanging Woman Creek, Wyom. & 1,210 & 35 \\
Tongue River at Stateline, Mont. & 3,753 & 34 \\
Prairie Dog Creek, Wyom. & 915 & 56 \\
Goose Creek, Wyom. & 1,180 & 52 \\
Tongue River near Monarch, Mont. & 1,134 & 49 \\
Powder River near Locate, Mont. & 33,849 & 38 \\
Little Powder River at Broadus, Mont. & 5,076 & 56 \\
Powder River at Moorhead, Mont. & 20,832 & 43 \\
Powder River at Sussex, Wyom. & 5,470 & 55 \\
Clear Creek near Arvada, Wyom. & 2,991 & 65 \\
Crazy Woman Creek near Arvada, Wyom. & 2,477 & 66 \\
\hline
\end{tabular}

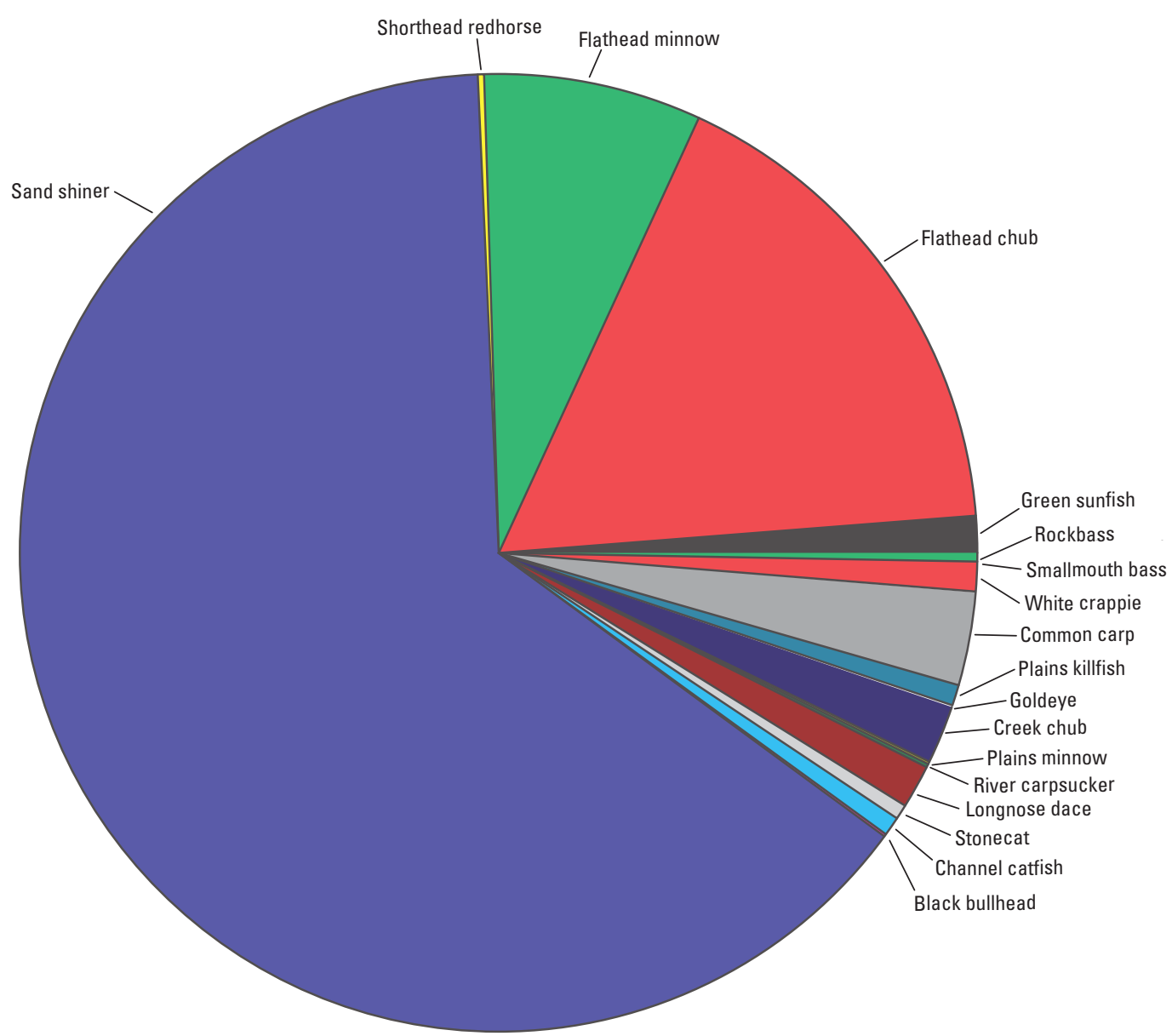

Figure 5-4. Proportion of each fish species captured in the Powder River drainage. Fish were collected by seining and electrofishing. 
non-native were not as clearly associated with the IBI scores. Some sites with large numbers of non-native fish species (such as Powder River at Sussex or Clear Creek) or few native fish species (Prairie Dog Creek) had relatively large IBI Scores.

In summary, white suckers and FHM were determined to be relatively abundant in the Tongue and Powder River drainages. Fish assemblages were similar to sampling completed in the Tongue and Powder River drainages by Stagliano (2006), Peterson and others (2009) and Peterson and others (2010). White suckers were the most common collected species in the Powder and Tongue River drainages. White suckers were also the most abundant species in the Tongue River drainage, but sand shiners were by far the most abundant species in the Powder River drainage.

\section{References Cited}

Barbour, M.T., Gerritsen, J., Snyder, B.D., and Stribling, J.B., 1999, Rapid Bioassessment protocols for use in streams and wadeable rivers - Periphyton, benthic macroinvertebrates and fish, (2d ed.): Washington, D.C., U.S. Environmental Protection Agency, Office of Water, EPA 841-B-99-002, 189 p.

Baxter, G.T., and Stone, M.D., 1995, Fishes of Wyoming, Cheyenne: Wyoming Game and Fish Department, 89 p.

Bramblett, R. G., Johnson, T. R., Zale, A.V., and, Heggem, D.G., 2005, Development and evaluation of a fish assemblage index of biotic integrity for northwestern Great Plains streams: Transactions of the American Fisheries Society, v. 134, p. $624-640$.

Clearwater, S.J., Morris, B.A., and Meyer, J.S., 2002, A comparison of coalbed methane product water quality versus surface water quality in the Powder River Basin of Wyoming, and an assessment of the use of standard aquatic testing organisms for evaluating the potential effects of coalbed methane product waters: Laramie, Wy., University of Wyoming, Department of Zoology and Physiology, Report to the Wyoming Department of Environmental Quality, 131 p.

Elser, A.A., Gorges, M.W., and Morris, L.M., 1980, Distribution of fishes in southeastern Montana: Helena, Mt., Montana Fish Wildlife and Parks, 111 p.

Grossman, G. L., Moyle, P.B., and Whitaker, J.O., 1982, Stochasticity in structural and functional characteristics of an Indiana stream fish assemblage - A test of community Theory: American Naturalist, v. 120, p. 423-454.

Holton, G.D, 1990, A field guide to Montana fishes: Helena, Montana Department of Fish Wildlife and Parks, 104 p.
Lee, D.S., Gilbert, C.R., Hocutt, C.H., Jenkins, R.E., McAllister, D.E., and Stauffer, J.R., Jr., 1980, Atlas of North American freshwater fish: Raleigh, North Carolina Museum of Natural History and the U.S. Fish and Wildlife Service, Publication \#1980-12, 854 p.

Lipsey, T.S.B, 2001, Using elevation, channel slope, and stream width to predict the occurrence of native warmwater fish species in the North Platte River drainage in Wyoming: Laramie, University of Wyoming, Master's Thesis, 238 p.

McCormick, S.D., 1993, Methods for nonlethal gill biopsy and measurement of $\mathrm{Na}+, \mathrm{K}+-$ ATPase activity: Canadian Journal of Fisheries and Aquatic Sciences, v. 50, p. 656-658.

Patton, T.M. 1997. Distribution and status of fishes in the Missouri River drainage in Wyoming - implications for identifying conservation areas: Laramie, Univeristy of Wyoming, Ph.D. Dissertation, $53 \mathrm{p}$.

Peterson, D.A., Wright, P.R.G., Edwards, P., Jr., Hargett, E.G., Feldman, D.L., Zumberge, J. R., and Dey, P., 2009, Ecological assessment of streams in the Powder River structural basin, Wyoming and Montana, 2005-06: U.S. Geological Survey Scientific Investigations Report 2009-5023, 139 p.

Peterson, D.A., Clark, M. A., Foster, K., Wright, D.L., and Boughton, J. K., 2010, Assessment of ecological conditions and potential effects of water produced from coalbed natural gas development on biological communities in streams of the Powder River structural basin, Wyoming and Montana: U.S. Geological Survey Scientific Investigations Report 2005-08, 2010, 84 p.

Poff, N.L. and Ward, J.V., 1989, Implications of streamflow variability and predictability for lotic community structure: A regional analysis of streamflow patterns: Canadian Journal of Fisheries and Aquatic Sciences, v. 46, p. 1,805-1,818.

Quist, M. C., Hubert, W. A., and Rahel, F.J., 2006, Concurrent assessment of fish and habitat in warmwater streams in Wyoming: Fisheries Management and Ecology, v. 13, p. 9-20.

Quist, M.C., Rahel, F.J., and Hubert, W.A., 2005, Hierarchical faunal filters - An approach to assessing effects of habitat and nonnative species on native fishes: Ecology of Freshwater Fish, v. 14, p. 24-39.

Stagliano, R. S., 2006, Aquatic Surveys and Assessment within the Middle Powder River Watershed: Miles City, Mt., Bureau of Land Management, ESA010009, Task Order \#28, 37 p. 


\section{Summary and Sample Calculations for Water-Quality Criteria}

By Aïda M.Farag, Don Skaar, and David D. Harper

\section{Chapter 6 of}

The Potential Effects of Sodium Bicarbonate, a Major Constituent of Produced Waters from Coalbed Natural Gas Production, on Aquatic Life

Edited by Aïda M. Farag and David D. Harper

Prepared in cooperation with Montana Fish, Wildlife, and Parks, U.S. Bureau of Land Management, and the U.S. Environmental Protection Agency

Scientific Investigations Report 2012-5008 


\section{Contents}

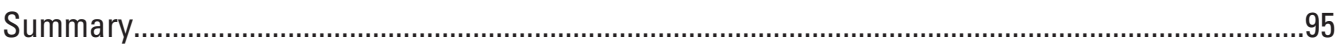

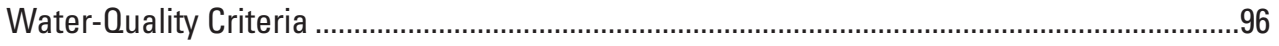

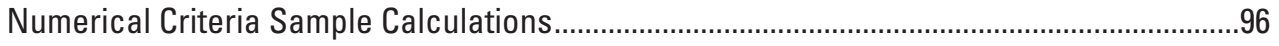

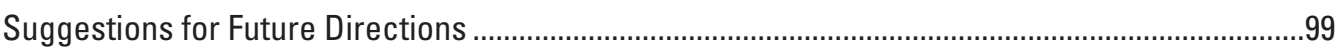

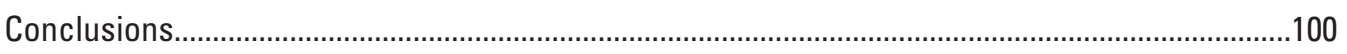

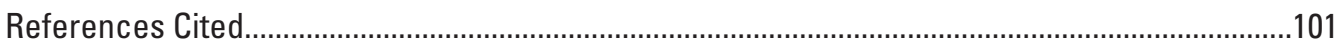

\section{Table}

6-1. The species geometric mean acute LC50 values and ranked sensitivity to sodium bicarbonate. Dilution waters were reconstituted to simulate Tongue, Powder and Yellowstone River water chemistry 


\title{
Summary and Sample Calculations for Water-Quality Criteria
}

\author{
By Aïda M.Farag', Don Skaar², and David D. Harper ${ }^{1}$
}

\section{Summary}

As is the case with any element or compound, there is a concentration where $\mathrm{NaHCO}_{3}$ will be toxic to aquatic life. A key to define the potential toxicity of any substance in the field is to complete controlled experiments in the laboratory and follow with field experiments and surveys to determine the potential exposure of aquatic life to toxic concentrations. The comparisons of all of these investigations will lead to a realistic interpretation of toxicological results.

In the previous chapters, acute toxicity to aquatic life was defined as a lethal concentration that will cause 50 percent mortality or detrimental effect at 48-hour (h) or 96-h (LC50 or EC50). The acute toxicity experiments were completed with a suite of organisms, including 7 species of fish, 5 species of invertebrates, and 1 amphibian species. Experiments performed on these multiple species resulted in LC50s that ranged from 1,120 to greater than (>) 8,000 milligrams sodium bicarbonate per liter $\left(\mathrm{mg} \mathrm{NaHCO}_{3} / \mathrm{L}\right)$ (also defined as 769 to $>8,000$ milligrams bicarbonate per liter $\left(\mathrm{mg} \mathrm{HCO}_{3}^{-/} / \mathrm{L}\right)$ or total alkalinity expressed as 608 to $>4,181$ milligrams calcium carbonate per liter $\left.\left(\mathrm{mg} \mathrm{CaCO}_{3} / \mathrm{L}\right)\right)$ that varied across species and lifestage within a species. The results were similar to those observed by Mount and others (1997) for Ceriodaphnia dubia (C. dubia) where they defined a 48-h LC50 of 1,020 mg $\mathrm{NaHCO}_{3} / \mathrm{L}$ compared to $1,288 \mathrm{mg} \mathrm{NaHCO} / \mathrm{L}$ observed during this study (both completed in moderately hard reconstituted water). Therefore, the current study provides results that are comparable to the limited amount data in the literature, and provides a broad application of the data because effects are defined on a wide range of species. As a result, managers located in watersheds with elevated concentrations of $\mathrm{NaHCO}_{3}$ will have benchmarks for comparisons of effects.

The chronic toxicity of $\mathrm{NaHCO}_{3}$ was defined in experiments that lasted from 7-60 days post-hatch. For these experiments, sublethal effects such as growth and reproduction, in addition to significant reductions in survival were included in the final determination of effects. We observed chronic toxicity at concentrations that ranged from 450 to 800

${ }^{1}$ USGS, Columbia Environmental Research Center, Jackson Field Research Station, Jackson, Wyoming.

${ }^{2}$ Montana Fish, Wildlife, and Parks, Helena, Montana. mg $\mathrm{NaHCO}_{3} / \mathrm{L}$ (also defined as 430 to $657 \mathrm{mg} \mathrm{HCO}_{3}^{-/} / \mathrm{L}$ or total alkalinity expressed as 354 to $539 \mathrm{mg} \mathrm{CaCO}_{3} / \mathrm{L}$ ) and the specific concentration depended on the sensitivity of the four species of invertebrates and fish exposed. Managers now also have data for chronic effects on multiple species.

Sublethal measurements such as sodium-potassium adenosine triphosphatase ( $\mathrm{Na} /$ ATPase) explained some effects at the individual level more completely, and defining these measurements in light of population level effects (survival) allows one to suggest how varying concentrations of $\mathrm{NaHCO}_{3}$ may affect fish. It appears that percent decrease in the activity of $\mathrm{Na} / \mathrm{K}$ ATPase and the age of the fish at the onset of the decrease may affect the ability of fathead minnow (Pimephales promelas) (FHM) to survive. For example, fish were able to survive an 8.12 percent decrease in $\mathrm{Na} / \mathrm{K}$ ATPase activity in the $400 \mathrm{mg} \mathrm{NaHCO} / \mathrm{L}$ treatment compared to the control when this decrease was first documented on day 60 . However, fish with an 8.50 percent decrease in $\mathrm{Na} / \mathrm{K}$ ATPase activity first documented on day 37 in the $500 \mathrm{mg} \mathrm{NaHCO} / \mathrm{L}$ treatment incurred significant reductions in survival and subsequently exhibited a 26.5 percent reduction in $\mathrm{Na} / \mathrm{K}$ ATPase at day 60. The effects of $\mathrm{NaHCO}_{3}$ on $\mathrm{Na} / \mathrm{K}$ ATPase activity and the ability of fish to survive likely are related to the age of the fish at exposure.

The acute laboratory results were substantiated with field experiments where early lifestage fish were held in live containers in situ for 96-h. The combination of in situ experiments, static-renewal experiments performed simultaneously with in situ experiments, and static renewal experiments performed with site water in the laboratory, demonstrated that coalbed natural gas $(\mathrm{CBNG})$ product water reduces survival of FHM and pallid sturgeon (Scaphirhynchus albus). Multiple tributary sites were associated with reduced survival and the concentrations of $\mathrm{NaHCO}_{3}$ at these sites were above the laboratory LC50 determined for the species experimented with in the field. Trace element analyses of water collected from the field did not reveal any other potential cause for the toxicity. However, it must be noted that trace organics were not measured in the water or sediments from these sites. At all but one site (Burger Draw), total ammonia concentrations were less than or equal to $(\leq) 0.5 \mathrm{mg} / \mathrm{L}$ and did not appear to be elevated to the extent to cause acute mortality. These results 
suggest that the concentrations of $\mathrm{NaHCO}_{3}$ in the tributary sites, except Burger Draw, caused the significant reductions in survival that were observed. Experiments were not performed on the main stem of the Powder or Tongue Rivers (except that the Powder River at Moorhead was used as a reference site). The mixing zone study provided evidence of substantial mixing near the outfalls to the main stem [800-1,200 meters (m) below the confluence] and also demonstrated a reduction in toxicity (based on survival) of water that was treated with ion exchange before release into the main stem of the Tongue River.

Data from the field experiments also defined a difference in sensitivity with age of the early lifestage fish. Differences were noted in sensitivity between 2-day-post-hatch (dph) and 6-dph FHM. As a result, care should be used when extrapolating results with fish that are greater than 2-dph to younger fish that seem to be more sensitive to $\mathrm{NaHCO}_{3}$. Measurements were not made of $\mathrm{Na} / \mathrm{K}$ ATPase in fish from experiments completed in situ, but the data from the laboratory experiments suggests that a significant decrease in $\mathrm{Na} / \mathrm{K}$ ATPase may have affected the ability of the 2-dph fish to survive in the field. Pallid sturgeon survival was adversely affected at 4- and 6-dph and suggests that pallid sturgeon are more sensitive to $\mathrm{NaHCO}_{3}$, regardless of age.

\section{Water-Quality Criteria}

Data provided in this report document a full array of effects generated by exposures to $\mathrm{NaHCO}_{3}$. Because $\mathrm{NaHCO}_{3}$ is a principle component in CBNG effluents, there is interest in defining the potential for $\mathrm{NaHCO}_{3}$ to affect aquatic life where surface discharge might occur. One approach to limit potential effects of $\mathrm{NaHCO}_{3}$ contained in effluents would be to establish numerical $\mathrm{NaHCO}_{3}$ water-quality criteria for the protection of aquatic life. Clear sets of policies, guidelines, and procedures to establish these criteria have been adopted by the U.S. Environmental Protection Agency (Stephan and others, 1985; Erickson and Stephan, 1988). Furthermore, once a sufficient database is generated to calculate criteria, data and decisions will be reviewed by the U.S. Environmental Protection Agency before a criterion would be adopted as part of a water-quality standard.

A numerical criterion is the most common, but is one of multiple types of criteria that may be implemented by States or Tribes. A numerical standard is appropriate when a specific element or chemical present in an effluent is known to cause toxicity. Other criteria that might be implemented include, narrative (may be based on whole effluent toxicity testing), biological (based on numbers and kinds of organisms expected to be present), nutrient (specifically limits nitrogen and phosphorous inputs to a system), and sediment criteria (U.S. Environmental Protection Agency 2011a, b, c).

Criteria are one part of a water-quality standard that might be implemented by States or Tribes. The derivation of overall water-quality standards includes three areas of concern (1) designations of use for water bodies, (2) establishment of criteria, and (3) guidelines related to anti-degradation (Gardner and Goss, 2009).

The purpose of the discussion that follows is to present sample calculations of numerical water-quality criteria using the database of toxicity evaluations that was generated in the previous chapters. This discussion will attempt to explain the specific calculations for numerical acute and chronic waterquality criteria and demonstrate how they might apply to $\mathrm{NaHCO}_{3}$. The final derivation and implementation of such criteria is, of course, left to the discretion of the concerned management agencies.

\section{Numerical Criteria Sample Calculation}

The focus of this study has been $\mathrm{NaHCO}_{3}$ and the sample calculations are first performed for this compound. However, standards often are established for simple elements and therefore additional calculations are provided for standards based on $\mathrm{HCO}_{3}{ }^{-}$because it is the suspected toxic fraction of the compound. Finally, calculations also are provided for alkalinity because it is a low cost and easily performed water-quality measurement. For this reason, its use can have wide spread applicability around this watershed and elsewhere. It should be noted that total alkalinity is expressed as " $\mathrm{CaCO}_{3} \mathrm{mg} / \mathrm{L}$." The reader is cautioned that although the measurement is expressed in this manner, $\mathrm{HCO}_{3}^{-}$is generally the main fraction of total alkalinity.

The criteria can be calculated to protect against acute or chronic effects of an element or compound. The calculation of an acute standard or criterion maximum concentration (CMC) involves several steps including: sensitivity ranking, calculations of geometric means, calculation of final acute value (FAV), and the final calculation of the CMC. Once an acceptable database of toxicity evaluations has been constructed, the responses (LC50 or EC50) of various species are compared to one another. Geometric means are calculated for each species then for each genus and the results are ranked to define the four most sensitive genera in the dataset. A total of 20 acute experiments that resulted in 13 genus mean or species mean acute values were performed during this study (table 6-1 and chapter 2, Results) (note to reader: Selenastrum capricornutum; plant species experiment data are not included and should be rerun, but does not appear more sensitive, so likely will not affect results and interpretation). The geometric means (table 6-1) for the four most sensitive genera are as follows.

1. Freshwater mussel (Lampsilis siliquoidea), LC50= $1,120 \mathrm{mg} \mathrm{NaHCO} / \mathrm{L}$.

2. Ceriodaphnia (Ceriodaphnia dubia) $\mathrm{LC} 50=$ $1,157 \mathrm{mg} \mathrm{NaHCO} / \mathrm{L}$.

3. Sturgeon (Scaphirhynchus), LC50 =1,377 mg $\mathrm{NaHCO}_{3} / \mathrm{L}$.

4. Fathead minnow (Pimephales promelas), $\mathrm{LC} 50=$ $1,906 \mathrm{mg} \mathrm{NaHCO} / \mathrm{L}$. 
Table 6-1. The species geometric mean acute LC50 values and ranked sensitivity to sodium bicarbonate. Dilution waters were reconstituted to simulate Tongue, Powder and Yellowstone River water chemistry.

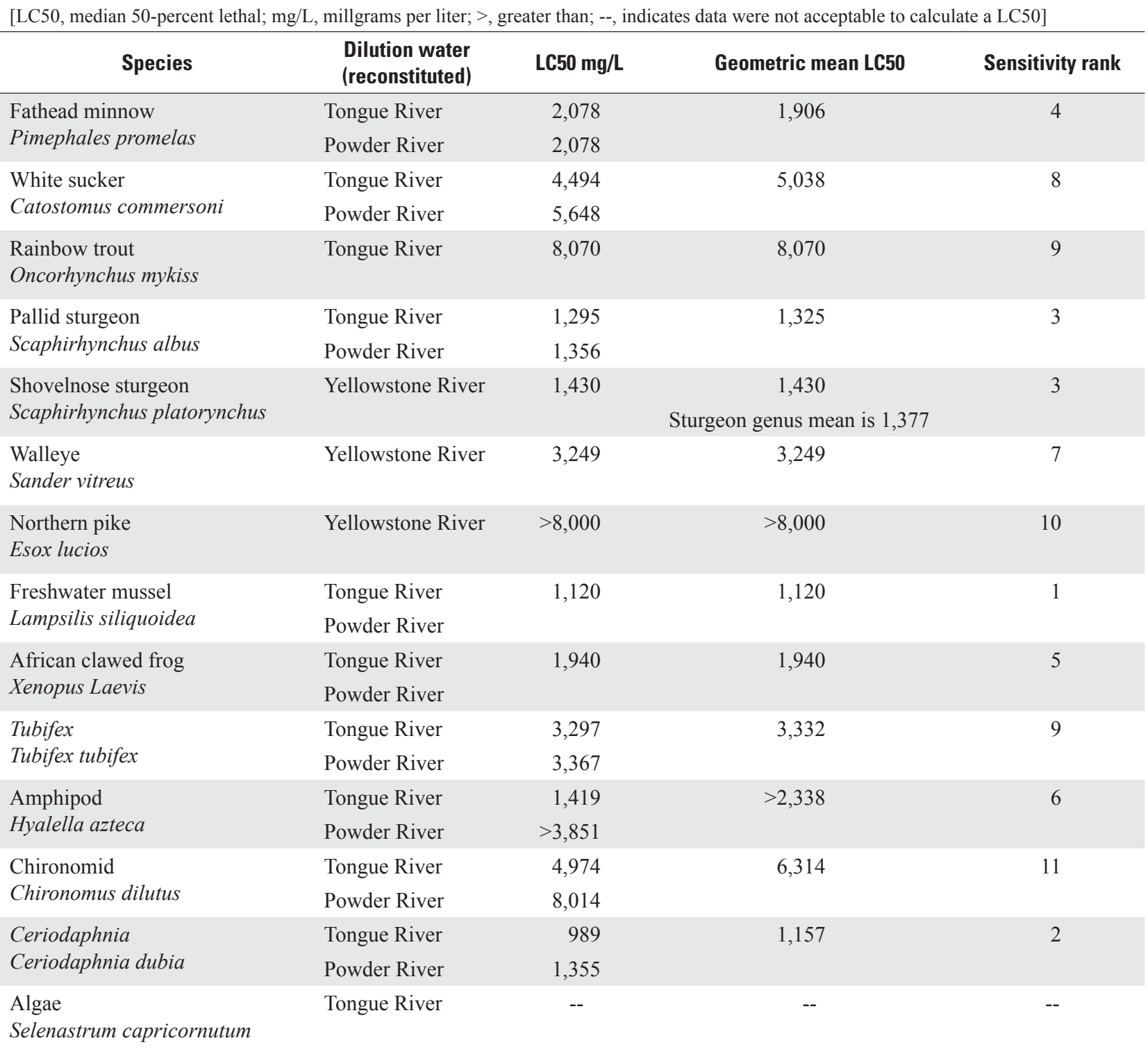

These four geometric means are used to determine an final acute value (FAV), which predicts sensitivity at the probability of 0.05 . Thus, data are extrapolated to protect 95 percent of all species. A computer program was used for this calculation (provided courtesy of U.S. Environmental Protection Agency and Bill Stubblefield, Parametrix Inc).

For this dataset, the $\mathrm{FAV}=918 \mathrm{mg} \mathrm{NaHCO}_{3} / \mathrm{L}$. The FAV is divided by two to account for the derivation based on 50 percent mortality (or other moribund observations, if an EC50 rather than an LC50 was used) (Stephan and others, 1985; Erickson, 1988). Therefore, using this dataset would provide a $\mathrm{CMC}=459 \mathrm{mg} \mathrm{NaHCO} / \mathrm{L}$.

This citerion mazimum concentration (CMC, also known as the acute criteria) defines the magnitude (for example, concentration) of $\mathrm{NaHCO}_{3}$ that would be allowed in an effort to protect aquatic life. However, this number would then need to be defined in terms of duration and frequency. Duration suggested by the U.S. Environmental Protection Agency is that the average in a 1-hr period should not exceed the suggested magnitude more than once every 3 years. However, the applicability of this recommendation would need to be investigated by concerned agencies.

To derive a chronic standard or Criterion Continuous Concentration (CCC), at least three acute/chronic ratios (ACR) must be calculated where the ratio between effects observed in a chronic experiment are compared with the acute effects observed in the same type of experimental water. For this dataset, the Inhibition Concentration (IC) that affects 20 percent 
of the sample population (IC20) chronic effect concentrations were as follows:

1. Fathead minnow (Pimephales promelas) $=527 \mathrm{mg}$ $\mathrm{NaHCO}_{3} / \mathrm{L}$; mean of 462 from experiment 1 (mortality) and 591 from 7-day (d) (growth)

2. C. dubia $=359 \mathrm{mg} \mathrm{NaHCO} / \mathrm{L}$ (reproductive effects)

3. Freshwater mussel (Lampsilis siliquoidea) $=952 \mathrm{mg}$ $\mathrm{NaHCO}_{3} / \mathrm{L}$ (moribund lack of foot movement).

The ACRs that result are as follows:

1. $\quad$ Fathead Minnow (Pimephales promelas) $1,749 / 527=3.32$.

2. C. dubia $1,288 / 359=3.59$.

3. Freshwater mussel (Lampsilis siliquoidea)1,120/952= 1.18 .

The geometric mean, or the Final Acute/Chronic Ratio $(\mathrm{FACR})=2.41$.

Finally, using this database, $\mathrm{CCC}=\mathrm{FAV} / \mathrm{FACR}=918 / 2.41=$ $381 \mathrm{mg} \mathrm{NaHCO}_{3} / \mathrm{L}$.

The CCC (also known as the chronic criteria) defines the magnitude of a continuous $\mathrm{NaHCO}_{3}$ concentration that would be allowed in an effort to protect aquatic life. However, this number would then need to be defined in terms of duration and frequency. Duration suggested by the U.S. Environmental Protection Agency is that the average concentration in a 24-hr period should not exceed the suggested magnitude more than once every 3 years. However, the applicability of this recommendation would need to be investigated by concerned agencies.

Below, the $\mathrm{CMC}$ and $\mathrm{CCC}$ are derived for $\mathrm{HCO}_{3}^{-}$and total alkalinity expressed as $\mathrm{mg} \mathrm{CaCO}_{3} / \mathrm{L}$. The geometric means for the same four genera expressed as $\mathrm{mg}^{-} \mathrm{HCO}_{3}^{-} / \mathrm{L}$ are as follows:

1. Freshwater mussel (Lampsilis siliquoidea), LC50= $844 \mathrm{mg} \mathrm{HCO}_{3}^{-/ 2}$.

2. C. dubia, $\mathrm{LC} 50=768 \mathrm{mg} \mathrm{HCO}_{3}^{-} / \mathrm{L}$.

3. Sturgeon (Scaphirhynchus), LC50 $=957 \mathrm{mg} \mathrm{HCO}_{3}^{-/ 2}$.

4. Fathead minnow (Pimephales promelas), LC50 = $1,334 \mathrm{mg} \mathrm{HCO}_{3}^{-} / \mathrm{L}$.

$\mathrm{FAV}=634$ and $\mathrm{CMC}=634 / 2=317 \mathrm{mg} \mathrm{HCO}_{3}{ }^{-} / \mathrm{L}$.

For this dataset, IC20 chronic effect concentrations were as follows:

1. Fathead minnow (Pimephales promelas $)=434 \mathrm{mg} \mathrm{HCO}_{3}^{-}$ /L; mean of 368 from Experiment 1 (mortality) and 499 from 7-d (growth).

2. C. $d u b i a=274 \mathrm{mg} \mathrm{HCO}_{3}^{-} / \mathrm{L}$ (reproductive effects).

3. Freshwater mussel (Lampsilis siliquoidea) $=$ $715 \mathrm{mg} \mathrm{HCO}_{3}^{-} / \mathrm{L}$ (moribund lack of foot movement).
The ACRs that result are as follows:

1. Fathead minnow (Pimephales promelas) $1,254 / 434=2.90$

2. C. dubia $842 / 274=3.07$

3. Freshwater mussel (Lampsilis siliquoidea) $844 / 715=1.18$

The geometric mean of the $\mathrm{ACR}=2.19$ and the $\mathrm{CCC}=$ $634 / 2.19=290 \mathrm{mg} \mathrm{HCO}_{3}^{-/}$L.

The geometric means as alkalinity expressed as $\mathrm{mg} \mathrm{CaCO}_{3} / \mathrm{L}$ are as follows:

1. Freshwater mussel (Lamsilis siliquoidea), LC50 = $670 \mathrm{mg} \mathrm{CaCO}_{3} / \mathrm{L}$.

2. C. dubia, $\mathrm{LC} 50=819 \mathrm{mg} \mathrm{CaCO}_{3} / \mathrm{L}$.

3. Sturgeon (Scaphirhynchus), LC50 = $794 \mathrm{mg} \mathrm{CaCO}_{3} / \mathrm{L}$.

4. Fathead minnow (Pimephales promelas), LC50 = $1,114 \mathrm{mg} \mathrm{CaCO}_{3} / \mathrm{L}$.

Thus, $\mathrm{FAV}=583$ and $\mathrm{CMC}=583 / 2=292 \mathrm{mg} \mathrm{CaCO}_{3} / \mathrm{L}$ total alkalinity.

For this dataset, IC20 chronic effect concentrations were as follows:

1. Fathead minnow (Pimephales promelas $)=$ $463 \mathrm{mg} \mathrm{CaCO} / \mathrm{L}$; mean of 302 from experiment 1 (mortality) and 424 from 7-d (growth).

2. C. $d u b i a=249 \mathrm{mg} \mathrm{CaCO}_{3} / \mathrm{L}$ (reproductive effects).

3. Freshwater mussel (Lamsilis siliquoidea) $=$ $605 \mathrm{mg} \mathrm{CaCO}_{3} / \mathrm{L}$ (moribund lack of foot movement).

The ACRs that result are as follows:

1. Fathead minnow (Pimephales promelas) $1,139 / 463=3.07$

2. C. dubia $846 / 249=3.29$

3. Freshwater mussel (Lamsilis siliquoidea) $670 / 605=1.12$.

The geometric mean of the $\mathrm{ACR}=2.24$ and the $\mathrm{CCC}=$

$583 / 2.24=260 \mathrm{mg} \mathrm{CaCO}_{3} / \mathrm{L}$ total alkalinity.

The identical four species/genus (fathead minnow, $C$. dubia, freshwater mussel, and sturgeon) were used as the four most sensitive for all calculations based on $\mathrm{NaHCO}_{3}, \mathrm{HCO}_{3}$, and total alkalinity. This was done for simplicity. However, if sensitivity comparisons are based on $\mathrm{HCO}_{3}{ }^{-}$and total alkalinity; the most sensitive genus changes slightly because amphipods and amphibians appear more sensitive with respect to these parameters.

Field validations and additional laboratory studies can be used to validate the acute and chronic findings. First, tributary sites were associated with reduced survival and the concentrations of $\mathrm{NaHCO}_{3}{ }^{-}$at these sites were more than the laboratory LC50 determined for the species experimented with in the field. Second, data from a chronic experiment performed with white suckers (Catostomus commersoni) revealed growth effects at $450 \mathrm{mg} \mathrm{NaHCO}_{3} / \mathrm{L}$. Histological effects also observed during the white sucker experiment supported these 
findings. These data were not included into CCC calculation because there was a large difference between the acute and chronic results for white suckers, which seemed to skew the ACR results (ACR for white suckers was $>10$ ). Regardless, the chronic experiment with white suckers point to an effect in the $500 \mathrm{mg} \mathrm{NaHCO} / \mathrm{L}$ range much like fathead minnow and C. dubia and validate the chronic findings.

In summary, a database of toxicity evaluations of $\mathrm{NaHCO}_{3}$ on aquatic life has been constructed. The experiments were performed with multiple species and were performed to meet U.S. Environmental Protection Agency acute and chronic guidelines established for the calculation of water-quality criteria. Using these data, acute and chronic criteria of 459 and $381 \mathrm{mg} \mathrm{NaHCO} / \mathrm{L}$, respectively, can be calculated for the protection of aquatic life. These numbers address the magnitude of concentrations, but additional considerations related to frequency and duration of allowable exceedance also would need to be considered. Finally, field experiments performed in tributaries in the Powder River Basin and an additional laboratory chronic experiment validate the findings and add credibility to the calculations performed.

Treatment practices employed in the Tongue/Powder River watershed appear to reduce the concentrations of $\mathrm{NaHCO}_{3}$ to less than the sample criteria calculations and the deionization treatment (Higgins Loop ${ }^{\mathrm{TM}}$, EMIT) appears to reduce the toxicity of untreated effluent water. Also, as of 2011, a treatment system has been located in the Beaver Creek tributary where toxicity was documented during this study. Although in situ bioassays have not been repeated at this site since the implementation of the treatment system, it is likely that toxicity of the tributary water is decreased if all inputs to the tributary are being treated with deionization.

\section{Suggestions for Future Directions}

The current body of work provided is substantial and provides data necessary to define the potential toxicity of $\mathrm{NaHCO}_{3}\left(\mathrm{HCO}_{3}^{-}\right.$, total alkalinity, conductivity) on aquatic life. The next steps would involve some refinement of the laboratory data, addition of species to the database, and monitoring of field sites as managers move to a monitoring and restoration phase of activities.

Although this volume contains an extensive body of work related to the effects of $\mathrm{NaHCO}_{3}$ on aquatic life, additional laboratory experiments could provide data to further refine the conclusions. There are several acute and chronic laboratory experiments that could further the research:

1. Selenastrum acute experiment-To further develop the acute database, a selenastrum experiment should be completed. The exposure completed during this study was completed successfully, but the samples collected for population counts were not readable with acceptable accuracy within the scope available. The researchers considered the selenastrum response that was observed to be less sensitive than the four species included in the final calculation of the sample acute criterion. However, these observations must be considered anecdotal and the sensitivity of selenastrum to $\mathrm{NaHCO}_{3}$ can only be definitively characterized with a successful experiment that includes accurate population counts.

2. Use of sensitive invertebrate species for acute experiment-During this study, Chironomus (midges), represented Class Insecta (table 1-1). Chironomus is a genus within the order Diptera. However, insects from the order Plecoptera (stoneflies) and Ephemeroptera (mayflies) often are more sensitive to toxicant exposures than Chironomus. Chironomus often is cultured in the laboratory and was an important first step to define the sensitivity of invertebrates to $\mathrm{NaHCO}_{3}$. As a next step, the sensitivity of Insecta could be further refined with the addition of an acute assay that utilizes a representative of Plecoptera or Ephemeroptera or both.

3. Additional mussel chronic experiment_-Freshwater mussels were among the four most sensitive species in the acute experiments completed during this study. A 10-d chronic experiment also was performed with freshwater mussels. The 10-d chronic experiment was performed with newly transformed freshwater mussels. When culturing freshwater mussels, newly transformed mussels are fairly robust and easy to work with in the laboratory. However, from 2 weeks after newly transformed until 2 months of age, the freshwater mussel cultures may encounter excessive mortalities and generally are not used as experimental animals. For this reason, a 10-d chronic experiment with newly transformed mussels (American Society of Testing and Materials International, 2006) was completed. The mussel culture was allowed to reach 2-months old, the age considered acceptable for use in a 28-d experiment (American Society of Testing and Materials International, 2006). However, the cultured animals did not survive until 2 months of age and the 28-d experiment was not completed. Although it is generally accepted that data from a $10-\mathrm{d}$ experiment will mimic that of a 28-d experiment, the U.S. Environmental Protection Agency may have some reservations with the use of the 10 - $d$ chronic data in the current sample calculations. For this reason, a 28-d chronic experiment with freshwater mussels is recommended to be performed with 2-month old mussels.

4. Ceriodaphnia dubia (C. dubia) chronic experiment in simulated Tongue River water-Sample calculations for chronic numerical criteria were performed with acute and chronic experiments completed with $C$. dubia performed in moderately hard reconstituted water. Although this is acceptable, an additional chronic experiment performed in simulated Tongue River water would strengthen the database and be similar to fathead minnow and freshwater mussel experimental water quality. 
5. White sucker acute/chronic experiment-The estimated percent hatch was 21 percent and control survival 54 percent at 30-d during the white sucker chronic experiment. These are small numbers but not unexpected because of experiments with the progeny of native fish that were spawned in the field. The progeny were brought into the laboratory for this experiment. The results during the white sucker chronic experiment were not unlike those observed during other chronic experiments. What was unusual was the large difference between acute and chronic effects noted for white sucker. For this reason, the acute chronic ratio was not included in the sample calculations provided. To make better use of the white sucker data two approaches are suggested. First, a white sucker acute experiment should be performed again. Depending on the results of this experiment, a second chronic experiment could be performed. Some initial research could be conducted to define the usefulness of a shorter chronic experiment to limit costs. However, the growth effects defined with white sucker were not observed until the end of the 60-d experiment and care must be taken to allow a chronic experiment sufficient time for observable effects to manifest.

6. Chronic experiment with additional species - If a shorter chronic experiment is found useful for white sucker, such experiments could also be used for additional species. For example, sauger is a much valued game species and acute and chronic experiments with this species would enhance the database. Sauger is a cannibalistic species which makes chronic experiments of a shorter duration desired so the experiment could end before this behavior manifests.

7. Use of additional amphibian species-The African clawed frog was used for this study because the timing necessary to collect frog eggs from the field for additional amphibian species was unattainable. Although feral populations of African clawed frogs exist in the United States, they are not native. The use of a strictly native frog species (for example, Leopard Frog) would add to this database. Additional field investigations could be performed to gather amphibian eggs for the laboratory studies. If field collected individuals are unavailable, there may be sources from hatchery programs (for example, redundant individuals from captive breeding programs) that might be available for breeding use during acute and chronic experiments.

8. Measurements of trace organics-Measurements of trace organics were not performed at the field sites or in the mixing zones. Trace organics could be measured in sediments or pore waters at these locations to define whether or not a source of organics is currently present as a potential pathway to the water column.
9. Monitoring - A monitoring program to provide an early alert to resource managers could include measurements of $\mathrm{NaHCO}_{3}$, alkalinity, and conductivity in water at key sites throughout the Tongue/Powder River watershed. These sites should be strategically placed to define the effects of effluent on critical tributaries (for example, Beaver Creek, Burger Draw) and the main stem of the rivers. When possible, sites should also include some locations within mixing zones. The monitoring strategy could include periodic, possibly a 3-5 year interval, assessments of the survival of aquatic life during in situ bioassays. These assays should include a plan where multiple species (fish, invertebrate, and amphibians) are studied to define the extent of effects on sensitive species, should they occur in the future.

\section{Conclusions}

A combination of in situ experiments, static-renewal experiments performed simultaneously with in situ experiments, and static renewal experiments performed with site water in the laboratory, demonstrated that coalbed natural gas (CBNG) product water reduces survival of fathead minnow and pallid sturgeon. More precisely, the survival of earlylifestage fish, especially those less than $(<) 6$-days post hatch (dph), likely is reduced significantly in the field when concentrations of $\mathrm{NaHCO}_{3}$ rise above $1,500 \mathrm{mg} / \mathrm{L}$. This conclusion is supported additionally by laboratory experiments.

A database of toxicity evaluations of $\mathrm{NaHCO}_{3}$ on aquatic life has been constructed. Using these data, acute and chronic criteria of 459 and $381 \mathrm{mg} \mathrm{NaHCO} / \mathrm{L}$, respectively, can be calculated for the protection of aquatic life. These concentrations are understandably less than $1,500 \mathrm{mg} \mathrm{NaHCO}_{3} / \mathrm{L}$ cited above because criteria are calculated to protect 95 percent of the most sensitive species and are calculated to protect beyond significant mortality.

Sublethal investigations revealed percent decrease in the activity of sodium-potassium adenosine triphosphatase $(\mathrm{Na} / \mathrm{K}$ ATPase, an enzyme involved in ionoregulation) and the age of the fish at the onset of the decrease may affect the ability of fathead minnow to survive exposures to $\mathrm{NaHCO}_{3}$. Increased sensitivity to $\mathrm{NaHCO}_{3}$ for younger fathead minnow (2-dph compared to 6-dph) also was documented during acute experiments. However, age was not a factor for pallid sturgeon and they were sensitive to product water regardless of age.

Treatment with the Higgins Loop ${ }^{\mathrm{TM}}$ technology and dilution of untreated water increased survival in the laboratory. Both of these situations reduced ammonia in addition to the concentrations of $\mathrm{NaHCO}_{3}$. These experiments addressed the acute toxicity of effluent waters being added to the main stem rivers, but did not address issues related to the volumes of water that may be added to the watershed.

Mixing zones of the three outfalls studied ranged from approximately $800-1,200 \mathrm{~m}$ below the confluence and the 
areas within these mixing zones with acutely lethal concentrations of $\mathrm{NaHCO}_{3}$ (as defined by the presence of concentrated dye) are limited and variable within each zone.

Areas with concentrations of $\mathrm{NaHCO}_{3}$ more than the concentrations likely to cause significant mortality, and more than the calculated sample water-quality criteria in the Tongue and Powder River Basins appear to be limited to tributaries and parts of mixing zones with considerable additions of untreated effluent.

Suggestions for future directions include performing additional acute and chronic experiments with additional species to further refine the database and beginning an effort to monitor for potential toxicity effects of $\mathrm{CBNG}$ product water in the field.

\section{References Cited}

American Society of Testing and Materials International, 2006, Standard guide for conducting laboratory toxicity tests with freshwater mussels: West Conshohocken, Pa., American Society of Testing and Materials International, E 2455-06, 52 p.

Erickson R.J., and Stephan, C.E., 1988, Calculation of the final acute value for water quality criteria for aquatic organisms - Duluth, U.S. Environmental Protection Agency, Environmental Research Laboratory, EPA/600/3-88/018, $63 \mathrm{p}$.

Gardner, T, H. and Goss, H., 2009, American Fisheries Society Short Course-Introduction to Water Quality Standards: accessed March 2009 at www.fisheries.org/units/wqs.

Stephan, C.E., Mount, D.I., Hansen, D.J., Gentile, J.H., Chapman G.A., and Brungs, W.A., 1985, Guidelines for deriving numerical national water quality criteria for the protection of aquatic organisms and their uses: Springfield, Va., Environmental Protection Agency, National Technical Information Service, PB85-227049, 54 p. Note: 2011 updated .pdf available at http://water.epa.gov/scitech/swguidance/standards/criteria/aqlife/upload/85guidelines.pdf.

U.S. Environmental Protection Agency, 2011a, Basic information, water quality criteria: accessed April 2010 at www.epa. gov/waterscience/criteria/basics.html.

U.S. Environmental Protection Agency, 2011b, What are water quality standards? Water quality criteria: accessed April 2010 at http://water.epa.gov/scitech/swguidance/standards/ crit.cfm.

U.S. Environmental Protection Agency, 2011c, Water quality criteria for nitrogen and phosphorous: accessed April 2010 at www.epa.gov/waterscience/criteria/nutrient/. 
Publishing support provided by:

Rolla Publishing Service Center

For more information concerning this publication, contact:

Director, USGS Columbia Environmental Research Center

4200 New Haven Road

Columbia, MO 65201

(573) 875-5399

Or visit the Columbia Environmental Research Center Web site at:

http://www.cerc.usgs.gov/ 

